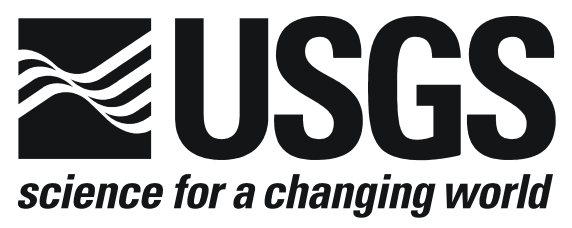

\title{
Magnetotelluric Data, Northern Frenchman Flat, Nevada Test Site, Nevada
}

By Jackie M. Williams, Brian D. Rodriguez, and Theodore H. Asch

\section{Open-File Report 2005-1240}

U.S. DEPARTMENT OF THE INTERIOR U.S. GEOLOGICAL SURVEY 


\section{U.S. Department of the Interior \\ Gale A. Norton, Secretary \\ U.S. Geological Survey
P. Patrick Leahy, Acting Director}

U.S. Geological Survey, Reston, Virginia 2005

Revised and reprinted: 2005

For product and ordering information:

World Wide Web: http://www.usgs.gov/pubprod

Telephone: 1-888-ASK-USGS

For more information on the USGS—the Federal source for science about the Earth, its natural and living resources, natural hazards, and the environment:

World Wide Web: http://www.usgs.gov

Telephone: 1-888-ASK-USGS 


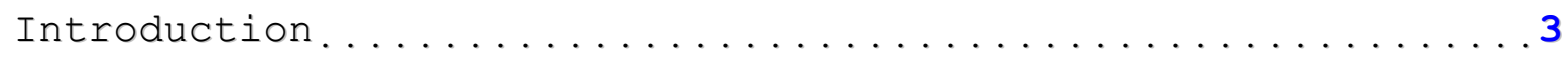

Magnetotelluric Method .....................3

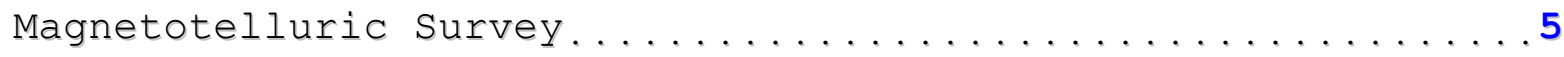

Magnetotelluric Data.....................6

References Cited.........................

Appendix: Magnetotelluric Data Plots................11

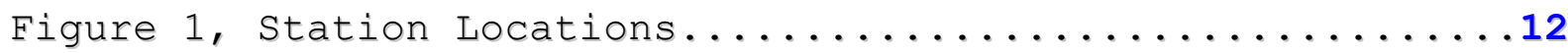




\section{Introduction}

Nuclear weapons are integral to the defense of the United States. The U.S. Department of Energy, as the steward of these devices, must continue to gauge the efficacy of the individual weapons. This could be accomplished by occasional testing at the Nevada Test Site (NTS) in Nevada, northwest of Las Vegas. Yucca Flat Basin is one of the testing areas at the NTS. One issue of concern is the nature of the somewhat poorly constrained pre-Tertiary geology and its effects on ground-water flow in the area subsequent to a nuclear test. Ground-water modelers would like to know more about the hydrostratigraphy and geologic structure to support a hydrostratigraphic framework model that is under development for the Yucca Flat Corrective Action Unit $(\mathrm{CAU})$.

During 2003, the U.S. Geological Survey (USGS) collected and processed Magnetotelluric (MT) and Audio-magnetotelluric (AMT) data at the Nevada Test Site in and near Yucca Flat to help characterize this pre-Tertiary geology. That work will help to define the character, thickness, and lateral extent of pre-Tertiary confining units. In particular, a major goal has been to define the upper clastic confining unit (UCCU) in the Yucca Flat area. Interpretation will include a three-dimensional (3-D) character analysis and two-dimensional (2-D) resistivity model. The purpose of this report is to release the MT sounding data for Frenchman Flat Profile 3, as shown in Figure 1. No interpretation of the data is included here.

\section{Magnetotelluric Method}

The MT method is a passive surface geophysical technique that uses the Earth's natural electromagnetic fields to investigate the electrical resistivity structure of the subsurface. The resistivity of geologic units is largely dependent upon their fluid content, porosity, degree of fracturing, temperature, and conductive mineral content (Keller, 1989). Saline fluids within pore spaces and fracture openings can reduce the resistivity of a resistive rock matrix. Resistivity also can be lowered by the presence of conductive clay minerals, carbon, and metallic mineralization. It is common for altered volcanic rocks to contain authigenic minerals that have resistivities of one tenth of those of the surrounding rocks (Nelson and Anderson, 1992). Increased temperatures cause higher ionic mobility and mineral activation energy, reducing rock resistivities significantly. Unaltered, unfractured igneous rocks are moderately to highly resistive (hundreds to thousands of ohm-m), whereas fault zones will show low resistivity (less than $100 \mathrm{ohm}-\mathrm{m}$ ) when they are composed of rocks that are fractured enough to have hosted fluid transport and consequent mineralogical alteration (Eberhart-Phillips and others, 1995). 
Carbonate rocks are moderately to highly resistive (hundreds to thousands of ohm-m) depending upon their fluid content, porosity, fracturing, and impurities. Marine shales, mudstones, and clay-rich alluvium normally are conductive (a few ohm-m to tens of ohm-m). Unaltered metamorphic rocks (non-graphitic) are moderately to highly resistive (hundreds to thousands of ohm-m). Tables of electrical resistivity for a variety of rocks, minerals, and geological environments are included in Keller (1987) and Palacky (1987).

The MT method can be used to probe the Earth's crust from depths of tens of meters to depths of tens of kilometers (Vozoff, 1991). Natural variations of the Earth's magnetic and electric field are measured and recorded at each MT station. The primary frequency bands used by the MT method are 10,000 to 1 hertz (Hz) from worldwide lightning activity and 1 to $0.0001 \mathrm{~Hz}$ from geomagnetic micro-pulsations. The natural electromagnetic waves propagate vertically in the Earth because the large resistivity contrast between the air and the Earth causes a vertical refraction of the electromagnetic wave transmitted into the Earth (Vozoff, 1972).

The natural electric and magnetic fields are recorded in two orthogonal, horizontal directions. The vertical magnetic field ("tipper") also is recorded. The resulting time-series signals are used to derive the tensor apparent resistivities and phases. First, the signals are converted to complex cross-spectra using Fast-Fourier-Transform (FFT) techniques. Then, least-squares, cross-spectral analysis (Bendat and Piersol, 1971) is used to solve for a transfer function that relates the observed electric fields to the magnetic fields with the assumption that the Earth consists of a two-input, two-output linear system. Prior to conversion to apparent resistivity and phase, the tensor normally is rotated. This rotation could be into principal directions that correspond to the direction of maximum and minimum apparent resistivity, or it may be made perpendicular to the survey traverses. For a two-dimensional (2-D) Earth, the MT fields then can be decoupled into a transverse electric (TE) mode (which uses the electric field perpendicular to the survey direction and the magnetic field parallel to the survey direction) and a transverse magnetic (TM) mode (which uses the electrical field parallel to the survey direction and the magnetic field perpendicular to the survey direction). Two-dimensional modeling generally is done to fit both modes. When the geology satisfies the 2-D assumption and the MT survey is perpendicular to the geologic strike, the MT data for the TE mode represent the electric field parallel to geologic strike, and the data for the TM mode represent the electric field across strike, along the survey direction. The MT method is well suited for studying complicated geological environments because the electric and magnetic relations are sensitive to vertical and horizontal variations in resistivity. The MT method is capable of establishing whether the 
electromagnetic fields are responding to subsurface terranes of effectively one, two, or three dimensions. An introduction to the MT method and references for a more advanced understanding are in Dobrin and Savit (1988) and Vozoff (1991).

\section{Magnetotelluric Survey}

From October to December of 2003, data were collected at 51 stations across the Yucca Flat Basin. The station locations were chosen to constrain the geologic/hydrostratigraphic interpretation, for proximity to roads, and to avoid, where possible, electrical noise from power lines and vehicles. The low frequency data $(0.0002$ to $200 \mathrm{~Hz})$ were collected with an Electromagnetic Instruments, Inc., (EMI) MT24/LF 24-bit system (EMI, 2002), and the high-frequency data (4 Hz to 23,000 Hz) were collected with a portable EMI MT-1 system (EMI, 1996). For the low frequency, horizontal electric fields were measured using three copper-copper sulfate porous pot electrodes placed in an L-shaped array with dipole lengths of 30 meters (m). Titanium electrodes were used in a similar array for the high-frequency data acquisition. The orthogonal, horizontal magnetic fields in the direction of the electric-field measurement array were sensed using EMI's high magnetic permeability, mu-metal-cored induction coils. For the low frequency data, two single-station recordings of the orthogonal, horizontal components of the electric and magnetic fields and the vertical magnetic field were acquired at Global Positioning System (GPS) referenced times and were used as remote references for each other. The high frequency data were recorded as non-remote referenced single stations.

The following table lists the 51 MT station locations as recorded using a GPS during field acquisition. Coordinates are referenced to the 1866 Clarke spheroid and North American 1983 Western United States datum. Longitude and latitude format is degrees, minutes, seconds. Universal Transverse Mercator (UTM) Zone 11 South and East units are in meters. Station elevation is given in meters. The accuracy of the $x, y, z$ component is $\pm 5 \mathrm{~m}$.

\begin{tabular}{|c|c|c|c|c|c|c|}
\hline Stations & Latitude & Longitude & Elevation & UTM 11S North & UTM 11S East & Remote \\
\hline $1 \mathrm{~m}$ & $36,53 ' 51.5$ & $116,11^{\prime} 50.3$ & 1456 & 4083613 & 571518 & 34 \\
\hline $2 m$ & $36,54^{\prime} 37.5$ & $116,10^{\prime} 21.7$ & 1373 & 4085052 & 573699 & None \\
\hline $3 m$ & $36,54^{\prime} 23.0$ & $116,08^{\prime} 57.9$ & 1304 & 4084623 & 575799 & None \\
\hline $4 m$ & $36,54^{\prime} 04.6$ & $116,07^{\prime} 37.5$ & 1254 & 4084071 & 577772 & 16 \\
\hline $5 m$ & $36,53 ' 27.0$ & $116,03^{\prime} 46.3$ & 1149 & 4082968 & 583504 & None \\
\hline $6 m$ & $36,53^{\prime} 36.9$ & $116,02^{\prime} 23.8$ & 1101 & 4083296 & 585543 & 36 \\
\hline $7 m$ & $36,53^{\prime} 52.5$ & $116,00^{\prime} 47.0$ & 1100 & 4083795 & 587935 & 30 \\
\hline $8 m$ & $36,54^{\prime} 18.2$ & $115,59 ' 08.6$ & 1229 & 4084622 & 590362 & None \\
\hline 9 & $36,57^{\prime} 36.4$ & $116,12 ' 18.9$ & 1536 & 4090540 & 570753 & 46 \\
\hline 10 & $36,58^{\prime} 01.6$ & 116,11'05.3 & 1437 & 4091331 & 572566 & 47 \\
\hline 11 & $36,58^{\prime} 44.4$ & $116,09 ' 53.3$ & 1445 & 4092663 & 574335 & 50 \\
\hline
\end{tabular}




\begin{tabular}{|c|c|c|c|c|c|c|}
\hline 12 & $36,59^{\prime} 36.6$ & $116,08^{\prime} 07.0$ & 1463 & 4094297 & 576949 & 49 \\
\hline 13 & $36,59 ' 25.0$ & $116,06^{\prime} 52.1$ & 1335 & 4093950 & 578802 & 48 \\
\hline 14 & $36,59 ' 27.5$ & $116,05^{\prime} 36.6$ & 1243 & 4094053 & 580670 & $48 a$ \\
\hline 15 & $36,57^{\prime} 34.8$ & $116,04^{\prime} 48.9$ & 1299 & 4090592 & 581884 & 51 \\
\hline 16 & $36,57^{\prime} 38.3$ & $116,04^{\prime} 02.2$ & 1236 & 4090710 & 583035 & 4 \\
\hline 17 & $36,57^{\prime} 26.7$ & $116,02^{\prime} 16.2$ & 1182 & 4090375 & 585659 & 29 \\
\hline 18 & $36,57 ' 26.5$ & $116,00 ' 34.3$ & 1186 & 4090400 & 588182 & None \\
\hline 19 & $36,57^{\prime} 22.4$ & $115,59^{\prime} 29.2$ & 1199 & 4090289 & 589792 & 35 \\
\hline 20 & $37,04^{\prime} 01.5$ & $116,12^{\prime} 12.2$ & 1528 & 4102410 & 570816 & 45 \\
\hline 21 & $37,03^{\prime} 20.9$ & $116,11^{\prime} 12.8$ & 1490 & 4101166 & 572297 & 44 \\
\hline 22 & $37,04{ }^{\prime} 02.3$ & $116,10^{\prime} 01.1$ & 1438 & 4102458 & 574055 & 43 \\
\hline 23 & $37,03 ' 23.6$ & $116,08 ' 16.2$ & 1347 & 4101290 & 576659 & 37 \\
\hline 24 & $37,03^{\prime} 06.3$ & $116,06 ' 52.0$ & 1306 & 4100781 & 578746 & 38 \\
\hline 25 & $37,02^{\prime} 54.4$ & $116,05^{\prime} 09.4$ & 1261 & 4100434 & 581280 & 39 \\
\hline 26 & $37,02^{\prime} 14.0$ & $116,04^{\prime} 05.3$ & 1240 & 4099202 & 582876 & 40 \\
\hline 27 & $37,01 ' 07.0$ & $116,02^{\prime} 00.7$ & 1209 & 4097167 & 585977 & 41 \\
\hline 28 & $37,01^{\prime} 06.3$ & $115,59^{\prime} 45.8$ & 1210 & 4097185 & 589311 & 42 \\
\hline 29 & $37,05^{\prime} 38.3$ & $116,09^{\prime} 55.2$ & 1501 & 4105419 & 574177 & 17 \\
\hline 30 & $37,04^{\prime} 54.5$ & $116,08^{\prime} 10.0$ & 1367 & 4104092 & 576786 & 7 \\
\hline 31 & $37,05^{\prime} 42.2$ & $116,06^{\prime} 51.2$ & 1334 & 4105583 & 578716 & None \\
\hline 32 & $37,05^{\prime} 41.0$ & $116,04^{\prime} 57.9$ & 1270 & 4105571 & 581515 & None \\
\hline 33 & $37,04 ' 56.9$ & $116,02^{\prime} 58.0$ & 1240 & 4104238 & 584489 & None \\
\hline 34 & $37,04{ }^{\prime} 07.5$ & $116,01 ' 20.1$ & 1241 & 4102745 & 586922 & 1 \\
\hline 35 & $37,04^{\prime} 04.8$ & $115,59 ' 59.5$ & 1265 & 4102681 & 588914 & 19 \\
\hline 36 & $37,04 ' 19.6$ & $115,58 ' 36.3$ & 1327 & 4103157 & 590961 & 6 \\
\hline 37 & $37,08^{\prime} 26.4$ & $116,08 ' 34.4$ & 1489 & 4110620 & 576125 & 23 \\
\hline 38 & $37,08^{\prime} 16.7$ & $116,06^{\prime} 16.5$ & 1331 & 4110353 & 579528 & 24 \\
\hline 39 & $37,07^{\prime} 46.8$ & $116,04^{\prime} 18.5$ & 1292 & 4109457 & 582448 & 25 \\
\hline 40 & $37,07^{\prime} 23.2$ & $116,02 ' 24.8$ & 1275 & 4108755 & 585262 & 26 \\
\hline 41 & $37,07^{\prime} 06.8$ & $116,01 ' 19.1$ & 1317 & 4108275 & 586891 & 27 \\
\hline 42 & $37,06^{\prime} 45.2$ & $115,59 ' 55.7$ & 1373 & 4107623 & 588955 & 28 \\
\hline 43 & $37,10^{\prime} 03.5$ & $116,08 ' 33.9$ & 1531 & 4113609 & 576110 & 22 \\
\hline 44 & $37,10^{\prime} 07.6$ & $116,08^{\prime} 00.0$ & 1486 & 4113742 & 576943 & 21 \\
\hline 45 & $37,13^{\prime} 13.1$ & $116,08^{\prime} 41.3$ & 1612 & 4119451 & 575875 & 20 \\
\hline 46 & $37,12^{\prime} 25.4$ & $116,07 ' 13.2$ & 1564 & 4118000 & 578059 & 9 \\
\hline 47 & $37,13^{\prime} 35.5$ & $116,04 ' 19.3$ & 1575 & 4120201 & 582325 & 10 \\
\hline 48 & $37,13^{\prime} 50.6$ & $116,03 ' 39.5$ & 1560 & 4120677 & 583300 & 13 \\
\hline 49 & $37,13^{\prime} 54.7$ & $116,02^{\prime} 25.7$ & 1486 & 4120819 & 585119 & 12 \\
\hline 50 & $37,13^{\prime} 12.2$ & $116,03 ' 52.7$ & 1536 & 4119490 & 582990 & 11 \\
\hline 51 & $37,11^{\prime} 50.5$ & $116,02^{\prime} 44.8$ & 1356 & 4116992 & 584688 & 15 \\
\hline
\end{tabular}

\section{Magnetotelluric Data}

The recorded time-series data were transformed to the frequency domain and processed to determine a 2-D apparent resistivity and phase tensor at each site. Rotation of the impedance tensor allows for decoupling into the TE and TM modes. During the analysis and interpretation process, each station is 
rotated to a fixed angle determined by the given nominal profile orientation. The data provided here have not been rotated from the original north-south, east-west acquisition orientation. Low frequency time series data were edited, and cross-power files were created with Egbert's (1997) multiple-station, magnetotelluric data-processing algorithms using remote references. Cross-power files were sorted to select optimal signal-to-noise time-series data sets (see Appendix at the back of this report).

The effects of near-surface resistivity anomalies can cause what are known as "static shifts" in the data (Sternberg and others, 1988). There were no significant static shifts in these data. Cultural features also can affect the measured magnetotelluric responses. These include fences, pipelines, communication lines, railways, and other manmade conductors.

The figures in the Appendix represent the field-processed MT data for each station (shown in boldface type in the table above) after the time-series data were converted to the frequency domain, and the tensor-transfer function was developed.

For each station, nine separate plots are given:

1. Apparent Resistivity ( $x$ and $y$ symbols are $x y$ and $y x$ components)

2. Impedance Phase ( $x$ and $y$ symbols are $x y$ and $y x$ components)

3. Rotation Angle

4. Impedance Skew

5. Multiple Coherency ( $x$ and $y$ symbols are $x y$ and $y x$ components)

6. Impedance Polar Plots

7. Tipper Magnitude

8. Tipper Strike

9. $\mathrm{HzHx}$ (x symbol) and HzHy (o symbol) Coherency

Error bars (], [) on the Apparent Resistivity, Impedance Phase, Skew, Tipper Magnitude, and Tipper Strike plots represent probable errors within one standard deviation of the sample variance (Gamble and others, 1979).

Apparent resistivity is the approximate ratio of the electric-field strength to the magnetic-field strength at a given frequency. The impedance phase is proportional to the slope of the apparent resistivity curve on a log-log plot, but from baselines at \pm 45 degrees (Vozoff, 1991). A measure of the dimensionality for MT data is provided by the impedance skew of the impedance tensor (Vozoff, 1972). If the effective measured resistivity response to the geology beneath a MT station truly is one or two dimensional, then the skew will be zero. Instrument and environmental sources of electrical noise can cause non-zero skew values. Skew values typically are small (about 0.1) for relatively low-noise recordings. Higher skews (above 0.2) are an indication of either the resistivity response to 3-D geology or higher levels of noise. Manmade electrical noise, such as power lines, power generators, and moving vehicles and trains, can have 
a negative effect on MT data quality. All of these local disturbances can produce incoherent noise that mainly affects frequencies above $1 \mathrm{~Hz}$. Other manmade electrical noise, such as direct current electric trains and active cathodic protection of pipelines, produces coherent electromagnetic signals that mainly affect frequencies below $1 \mathrm{~Hz}$.

In the survey area, noise from a number of small power lines and small moving vehicles was negligible at distances greater than $0.4 \mathrm{~km}$ from the noise source. Power-line signal levels were measured at each site and typically were less than 20 percent of the maximum recordable signals. Noise from larger power lines, power generators, pipelines, and trains was negligible at distances greater than $5 \mathrm{~km}$. Local lightning, wind, and rainstorms also can degrade data quality. Burying the magnetic induction coils and the electric dipole wires minimized wind noise.

Predicted values of the electric field can be computed from the measured values of the magnetic field (Vozoff, 1991). The coherence of the predicted electric field with the measured electric field is a measure of the signal-to-noise ratio provided in the multiple coherency plots. Values are normalized between 0 and 1; values at 0.5 signify signal levels equal to noise levels. For this data set, coherencies generally were at an acceptable level, except at times in the frequency ranges of 0.01 to $5 \mathrm{~Hz}$ (often referred to as the "dead band").

The field-processed MT data include some scatter and poor signal-to-noise ratios. Spectral results were inspected visually for noisy data, and the best signal-to-noise field data were combined into the final plots.

The magnetotelluric impedance polar plots provide a measure of MT data dimensionality (Reddy and others, 1977). For 1-D resistivity structures, the principal impedance polar diagram (dashed line) is a circle. For 2-D or 3-D resistivity structures, the principal impedance polar diagram (dashed line) elongates either parallel or perpendicular to strike direction. Over resistors, the principal impedance polar diagram elongates perpendicular to strike direction, and over conductors, it elongates parallel to strike direction. For 2-D resistivity structures, the additional impedance polar diagram (solid line) attains the shape of a symmetric clover leaf. For 3-D resistivity structures, the additional impedance polar diagram (solid line) elongates in one direction, and its amplitude is comparable to that of the principal impedance polar diagram (dashed line).

The magnetotelluric "tipper" is calculated from the vertical component of the magnetic field. The tipper magnitude is a measure of the "tipping" of the magnetic field out of the horizontal plane (Vozoff, 1991). It will be equal to zero for the 1-D case. It typically increases to values between 0.1 to 0.5 and seldom approaches 1 , as it responds primarily to vertical 
and subvertical structures. The tipper magnitude of the stations discussed in this report ranged from 0.1 to 0.6 over the lower frequencies, indicating some vertical structure at depth. The tipper strike is used to help resolve the 90-degree ambiguity in the impedance rotation angle. The $\mathrm{HzHx}$ and $\mathrm{HzHy}$ coherency is a measure of the signal-to-noise ratio of the vertical magnetic field with respect to each of the orthogonal, horizontal magnetic-field directions. Values are normalized between 0 and 1; values at 0.5 signify signal levels equal to noise levels. These three-component magnetic-field coherencies provide a check on the signal-to-noise ratio of the measured values in the tipper magnitude and tipper strike plots.

\section{References Cited}

Bendat, J.S., and Piersol, A.G., 1971, Random data-analysis and measurement procedures: New York, Wiley Interscience, $407 \# \mathrm{p}$.

Dobrin, M.D., and Savit, C.H., 1988, Introduction to geophysical prospecting (4th ed.): New York, McGraw-Hill, $867 \# \mathrm{p}$.

Eberhart-Phillips, Donna, Stanley, W.D., Rodriguez, B.D., and Lutter, W.J., 1995, Surface seismic and electrical methods to detect fluids related to faulting: Journal of Geophysical Research, v. 100, no. B7, p. 12919-12936.

Egbert, G.D., 1997, Robust multiple station magnetotelluric data processing: Geophysics Journal International, 130, p. 475-496.

EMI, 1996, MT-1 magnetotelluric system operation manual, version 3.2: Richmond, Calif., ElectroMagnetic Instruments, Inc., $220 \# p$.

EMI, 2002, MT24/LF System Operation and Maintenance Manual, version 1.0: Richmond, Calif., ElectroMagnetic Instruments, Inc., $72 \#$ p.

Gamble, T.D., Goubau, W.M., and Clarke, J., 1979, Error analysis for remote reference magnetotellurics: Geophysics, v. 44, no. 5, p. 959-968.

Keller, G.V., 1987, Rock and mineral properties, in Nabighian, M.N., ed., Electromagnetic methods in applied geophysics theory: Tulsa, Okla., Society of Exploration Geophysicists, v. 1, p. 13-51. 
Keller, G.V., 1989, Electrical properties, in Carmichael, R.S., ed., Practical handbook of physical properties of rocks and minerals: Boca Raton, Fla., CRC Press, p. 359-427.

Nelson, P.H., and Anderson, L.A., 1992, Physical properties of ash flow tuff from Yucca Mountain, Nevada: Journal of Geophysical Research, v. 97, no. B5, p. 823-6841.

Palacky, G.J., 1987, Resistivity characteristics of geologic targets, in Nabighian, M.N., ed., Electromagnetic methods in applied geophysics theory: Tulsa, Okla., Society of Exploration Geophysicists, v. 1, p. 53-129.

Reddy, I.K., Rankin, David, and Phillips, R.J., 1977, Three-dimensional modelling in magnetotelluric and magnetic variational sounding: Geophysics Journal of the Royal Astronomical Society, v. 51, p. 313-325.

Sternberg, B.K., Washburne, J.C., and Pellerin, Louise, 1988, Correction for the static shift in magnetotellurics using transient electromagnetic soundings: Geophysics, v. 53, p.1459-1468.

Vozoff, Keeva, 1972, The magnetotelluric method in the exploration of sedimentary basins: Geophysics, v. 37, p. $980-141$.

Vozoff, Keeva, 1991, The magnetotelluric method, in Nabighian, M.N., Electromagnetic methods in applied geophysics: Tulsa, Okla., Society of Exploration Geophysicists, v. 2, pt. B, p. 641-711. 


\section{Appendix}

\section{Magnetotelluric Data Plots}

There are nine separate plots for each station:

1. Apparent Resistivity for the rotated maximum (x symbol) and minimum (o symbol) modes

2. Impedance Phase for the rotated maximum ( $x$ symbol) and minimum (o symbol) modes

3. Rotation Angle for the impedance tensor (corresponds to the direction of maximum apparent resistivity)

4. Impedance skew for the impedance tensor

5. Multiple Coherency for the rotated maximum (x symbol) and minimum (o symbol) modes of the electric field

6. Impedance Polar Plots (at 12 selected frequencies)

7. Tipper Magnitude for the vertical magnetic field

8. Tipper Strike for the vertical magnetic field

9. $\mathrm{HzHx}$ (x symbol) and HzHy (o symbol) Coherency

Refer to the "Magnetotelluric Data" section in this report for an explanation of these plots. The "priorities" listed on the plots were determined prior to data acquisition. During post-processing the priority ranking was changed. 


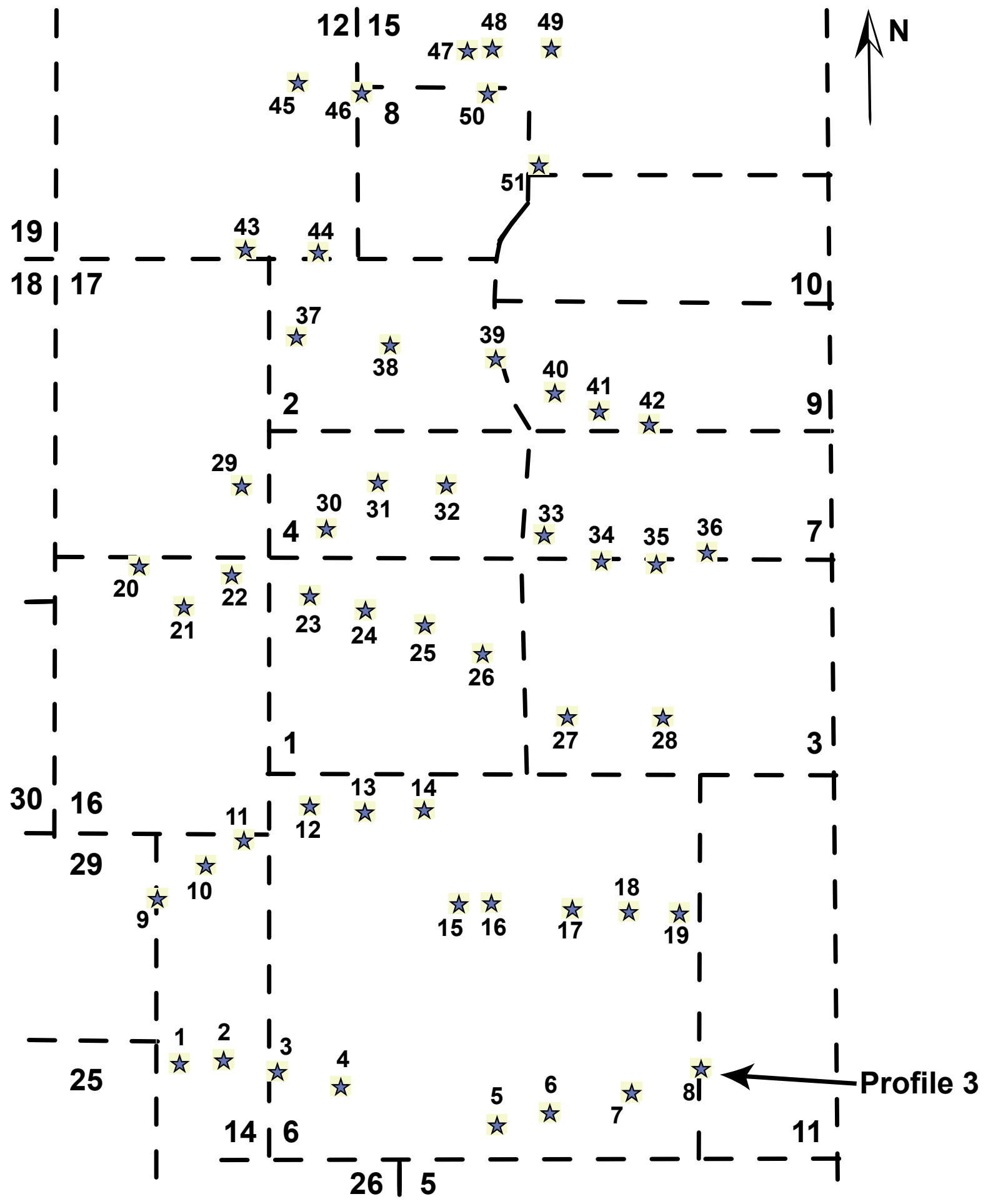

Figure 1. Index map showing magnetotelluric (MT) stations in and near Yucca Flat, Nevada. MT stations acquired in 2003 are numbered stars 1-51. Dashed lines are Nevada Test Site Areas 1-30. 


\section{APPARENT RESISTIVITY}

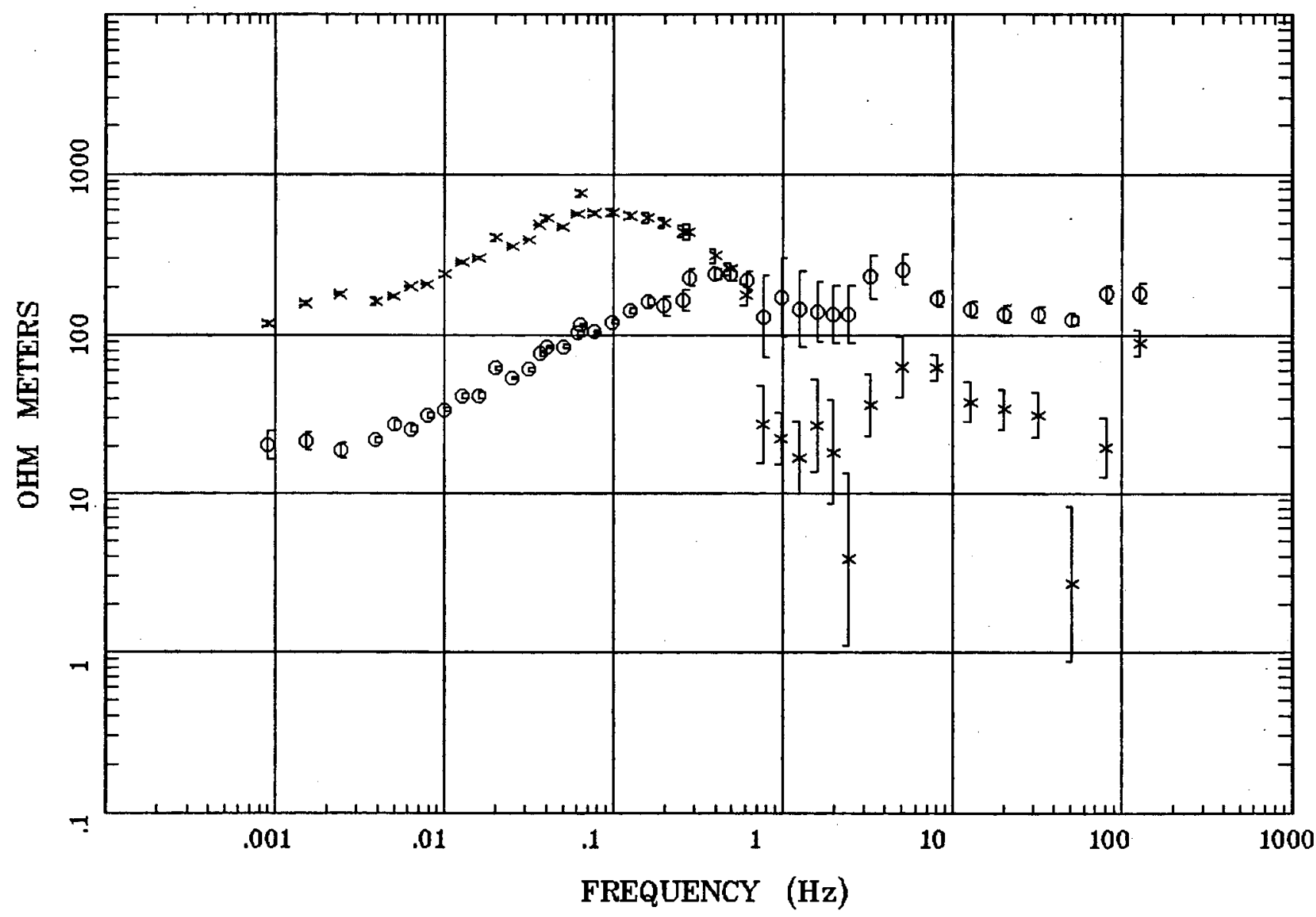

Client:

Remote:

Acquired: 19:5 Mar 08, 1998

Survey Co:
Rotation:

Filename: s1r34.a01

Channels: Ch1 Ch2 Ch3 Ch4 Ch5 Ch3 Ch4 Plotted: 10:56 Dec 07, 2004

$<$ EMI - ElectroMagnetic Instruments > 


\section{IMPEDANCE PHASE}

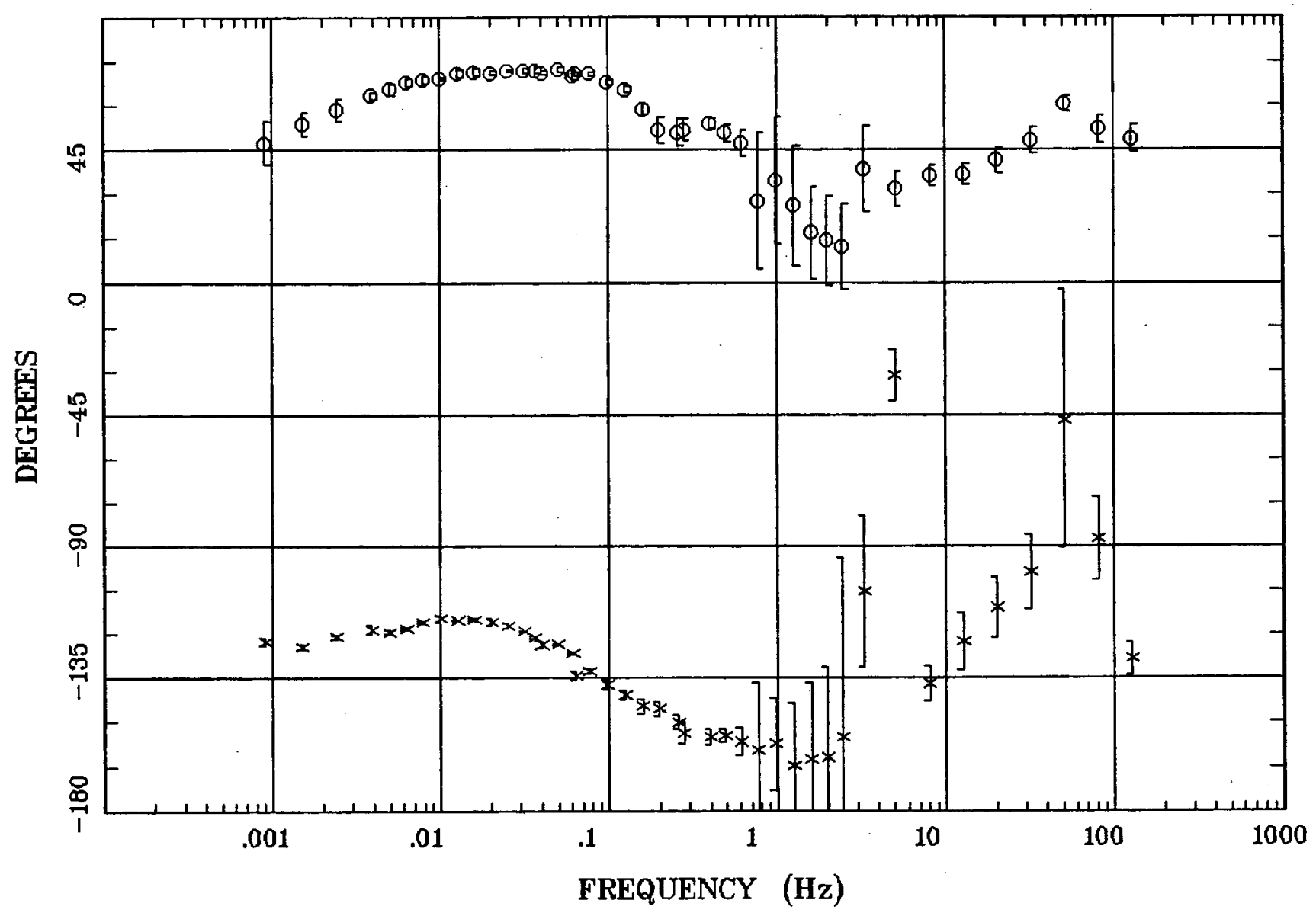

Client:

\section{Remote:}

Acquired: 19:5 Mar 08, 1998 Survey Co:
Rotation:

Filename: s1r34.a01

Channels: Ch1 Ch2 Ch3 Ch4 Ch5 Ch3 Ch4

Plotted: 10:56 Dec 07, 2004

< EMI - ElectroMagnetic Instruments > 
Station 1

\section{ROTATION ANGLE}

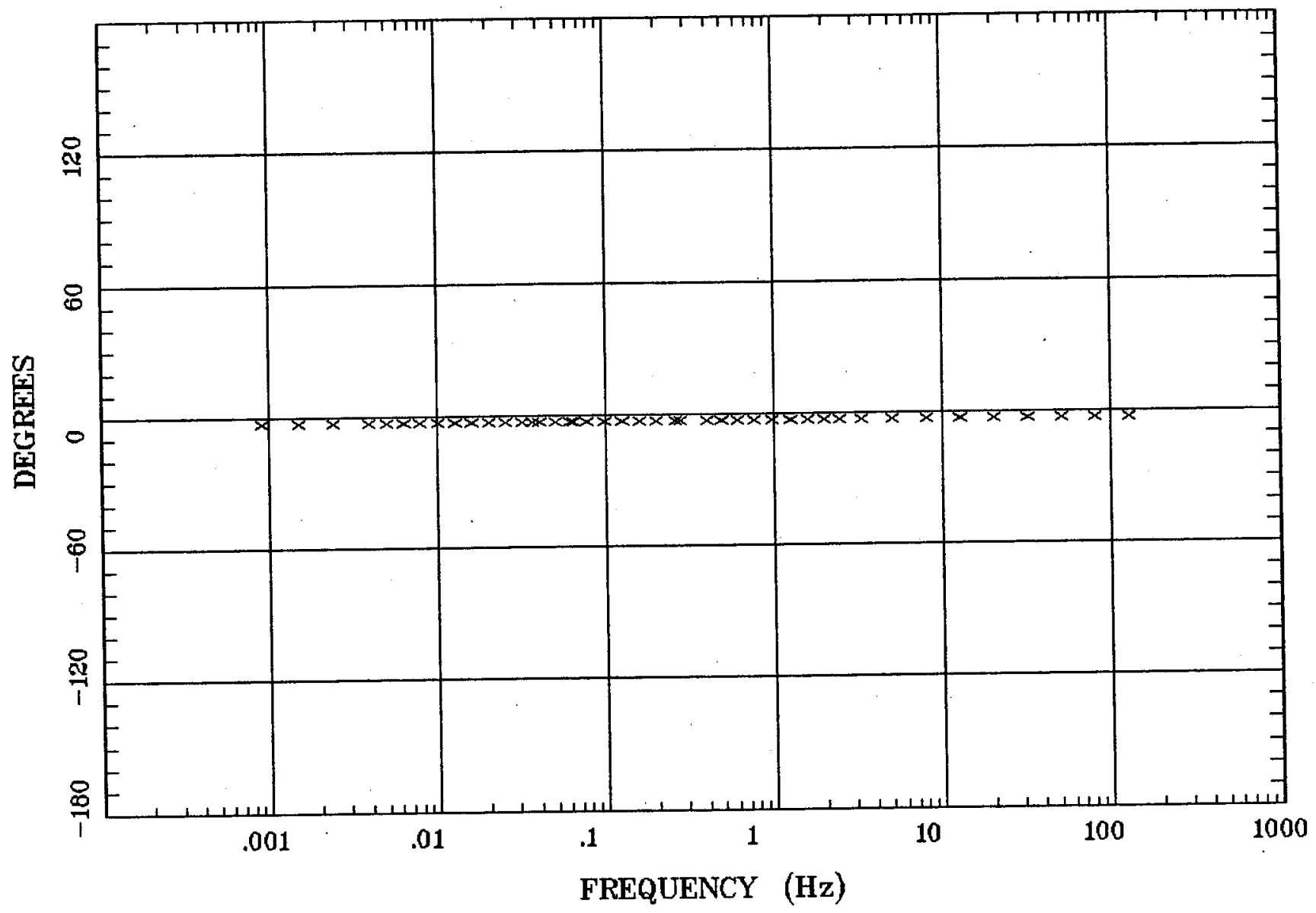

Client:

Remote:

Acquired: 19:5 Mar 08, 1998

Survey Co:
Rotation:

Filename: s1r34.a01

Channels: Ch1 Ch2 Ch3 Ch4 Ch5 Ch3 Ch4

Platted: 10:56 Dec 07, 2004

$<$ EMI - ElectroMagnetic Instruments > 
IMPEDANCE SKEW

Station 1

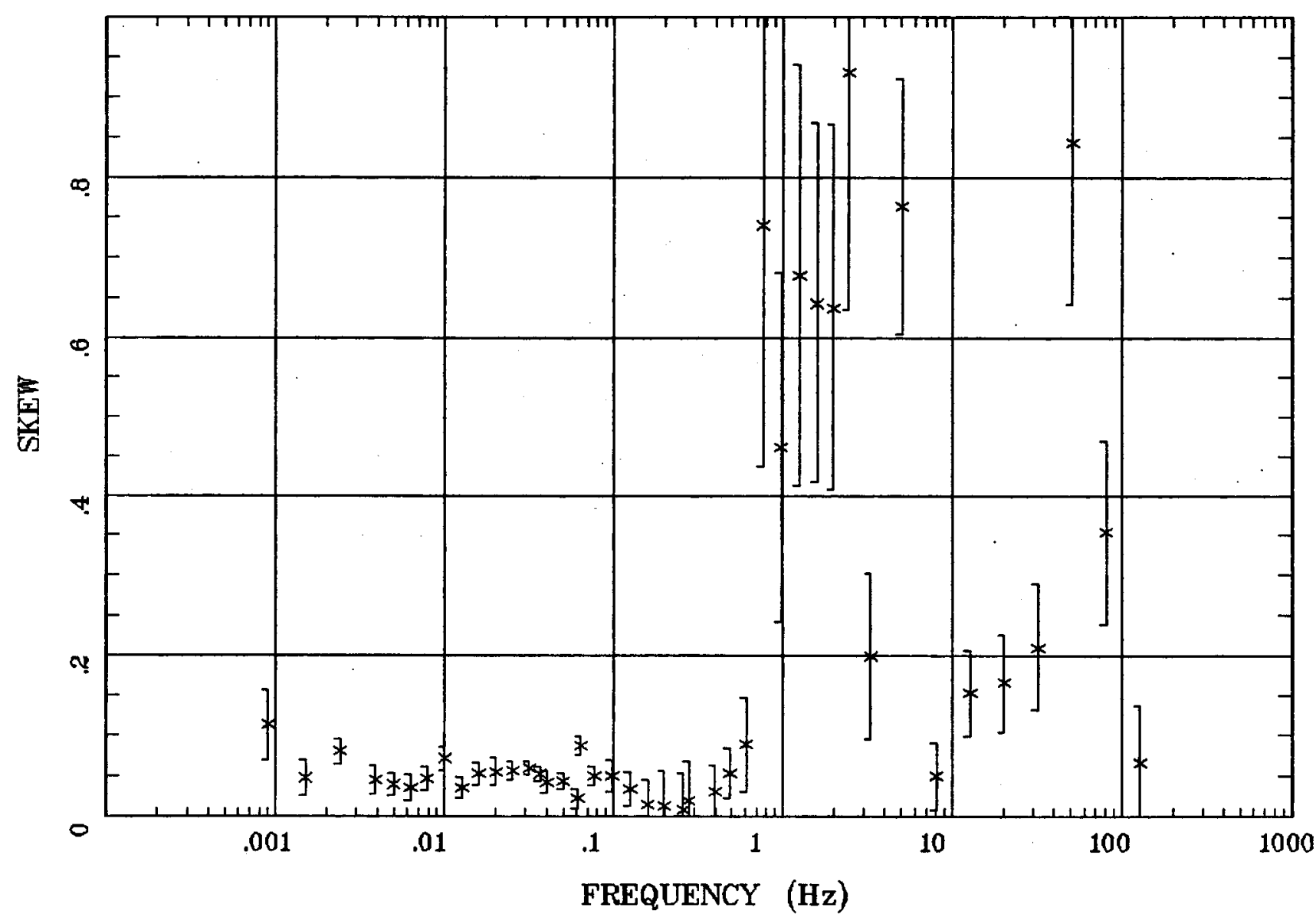

Client:

Remote:

Acquired: 19:5 Mar 08, 1998 Survey Co:
Rotation:

Filename: s1r34.a01

Channels: Ch1 Ch2 Ch3 ch4 Ch5 Ch3 Ch4 Plotted: 10:56 Dec 07, 2004

$<$ EMI - ElectroMagnetic Instruments > 
E MULT Coh.

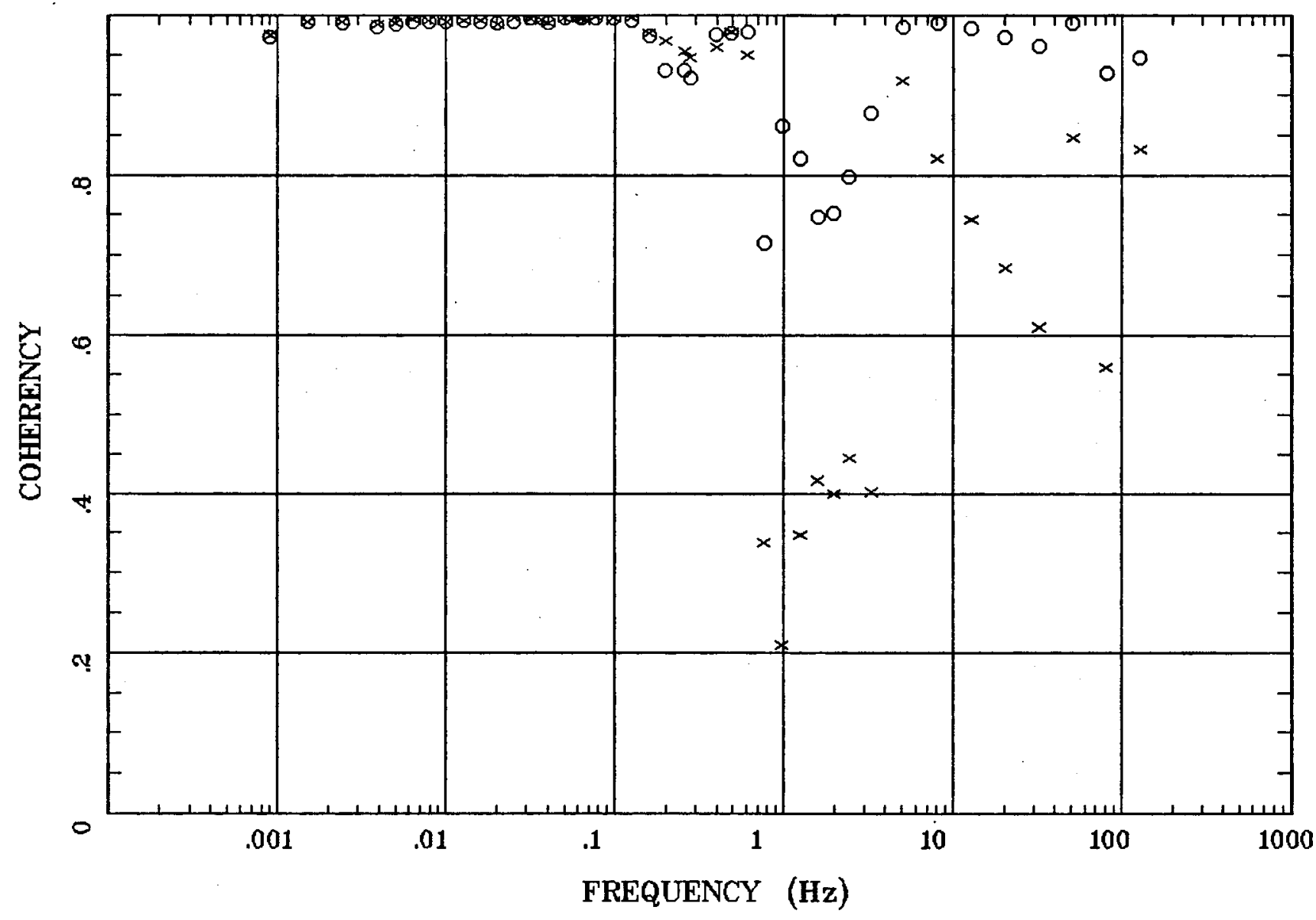

Client:

Remote:

Acquired: 19:5 Mar 08, 1998 Survey Co:
Rotation:

Filename: s1r34.a01

Channels: Ch1 Ch2 Ch3 Ch4 Ch5 Ch3 Ch4

Plotted: 10:56 Dec 07, 2004

< EMI - ElectroMagnetic Instruments > 


\section{POLAR PLOTS}

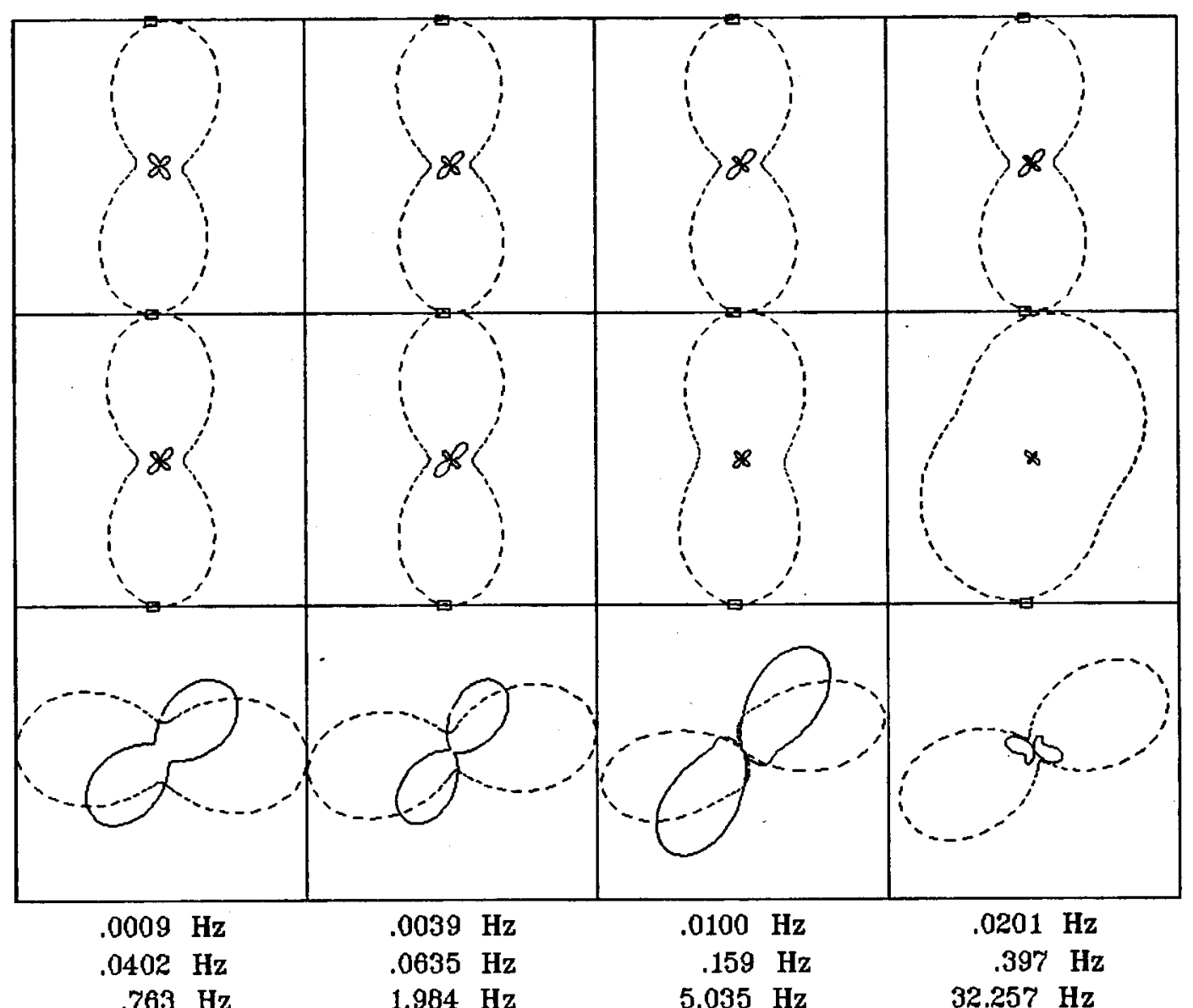

Client:

Remote:

Acquired: 19:5 Mar 0B, 1998 Survey Co:
Rotation:

Filename: s1r34.001

Channels: Ch1 Ch2 Ch3 Ch4 Ch5 Ch3 Ch4

Platted: 10:56 Dec 07, 2004

$<$ EMI - ElectroMagnetic Instruments 


\section{TIPPER MAGNITUDE}

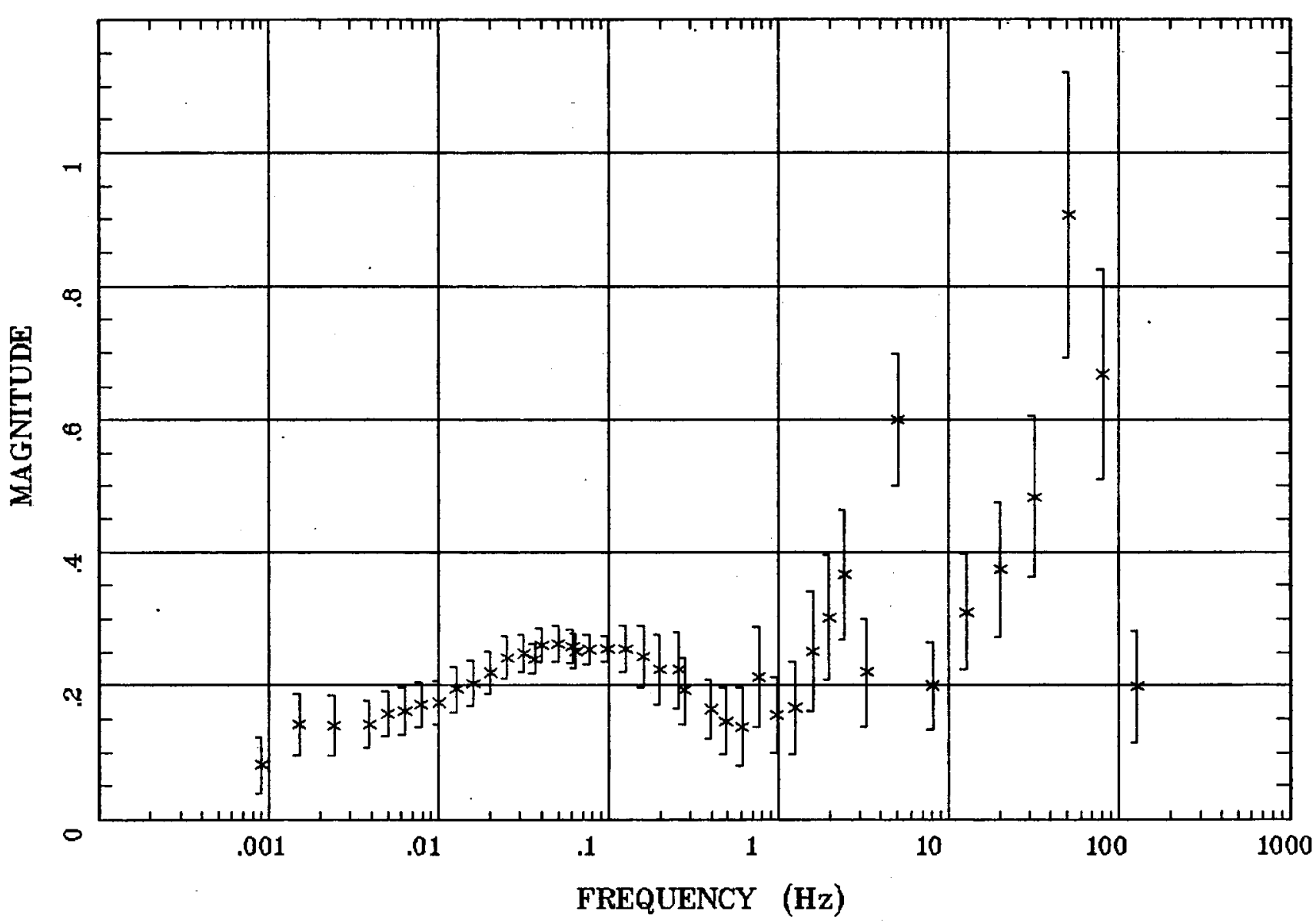

Client:

Remote:

Acquired: 19:5 Mar 08, 1998 Survey Co:
Rotation:

Filename: s1r34.a01

Channels: Ch1 Ch2 Ch3 Ch4 Ch5 Ch3 Ch4 Plotted: 10:56 Dec 07, 2004

< EMI - ElectroMagnetic Instruments > 


\section{TIPPER STRIKE}

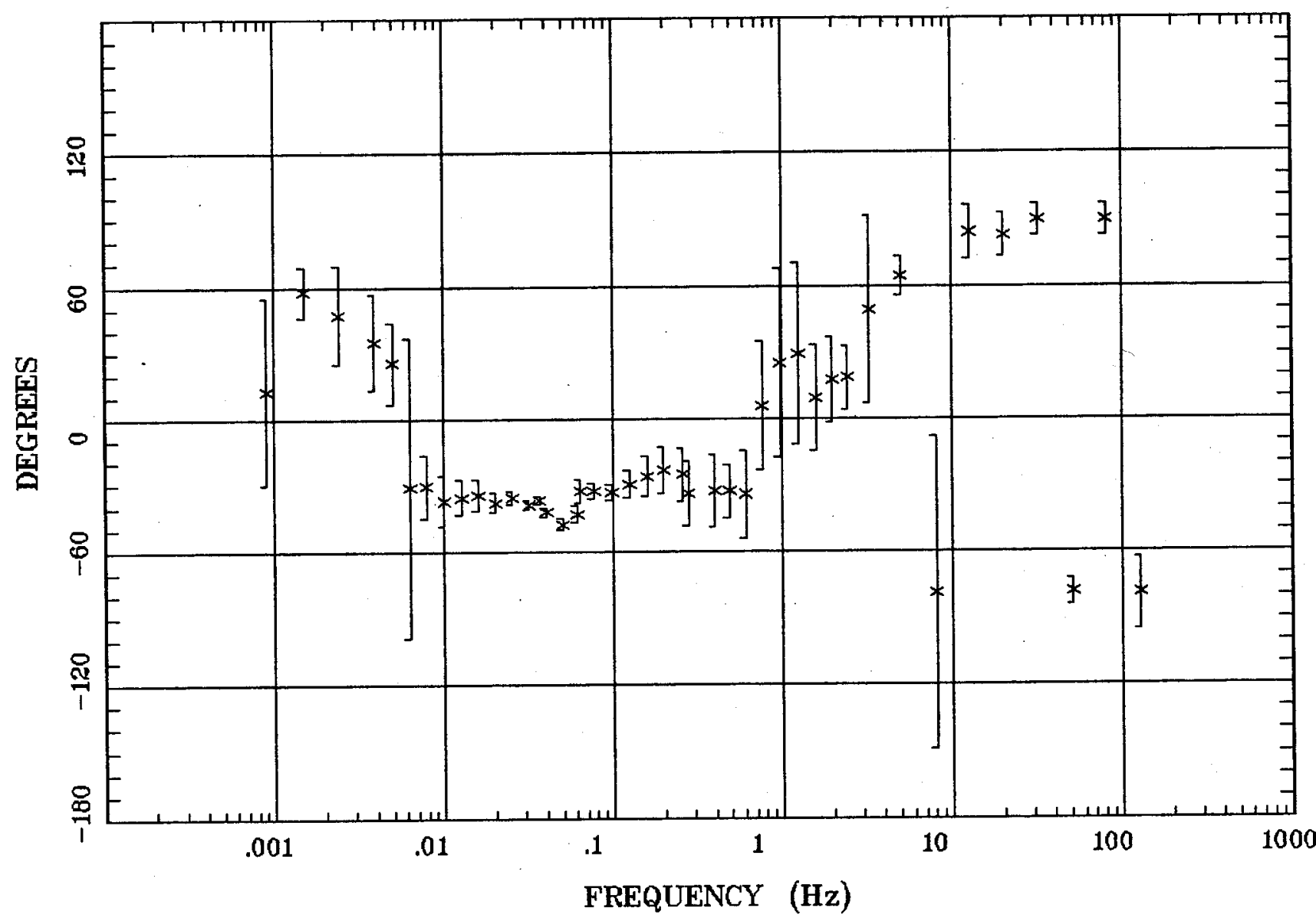

Client:

Remote:

Acquired: $19: 5$ Mar 08, 1998 Survey Co:
Rotation:

Filename: s1r34.a01

Channels: Ch1 Ch2 Ch3 Ch4 Ch5 Ch3 Ch4

Plotted: 10:56 Dec 07, 2004

< EMI - ElectroMagnetic Instruments 
HzHx.x Coh HzHy.o

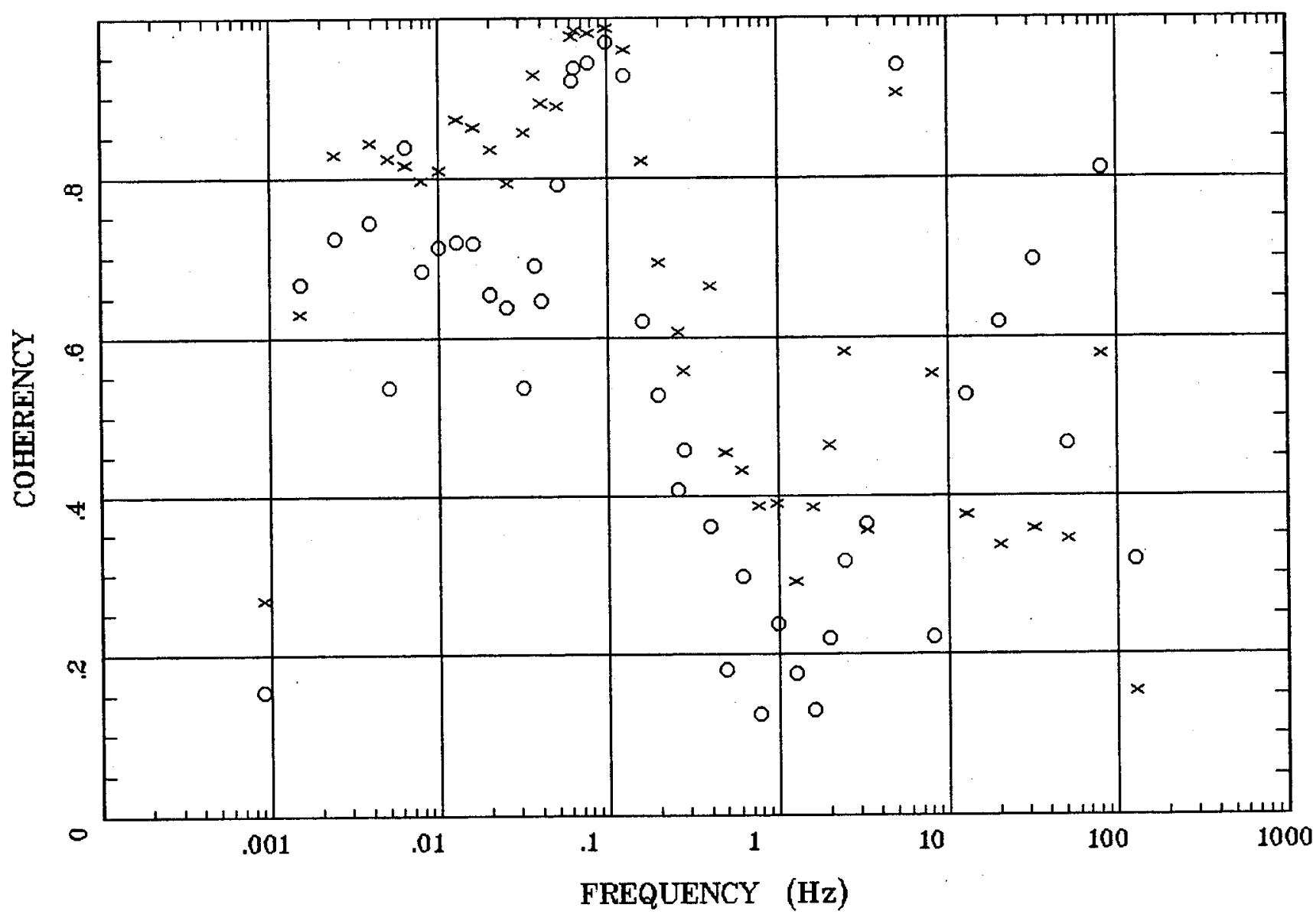

Client:

Remote:

Acquired: 19:5 Mar 08, 1998 Survey Co:
Rotation:

Filename: s1r34.a01

Channels: Ch1 Ch2 Ch3 Ch4 Ch5 Ch3 Ch4 Plotted: 10:56 Dec 07, 2004

$<$ EMI - ElectroMagnetic Instruments 


\section{APPARENT RESISTIVITY}

Station 2

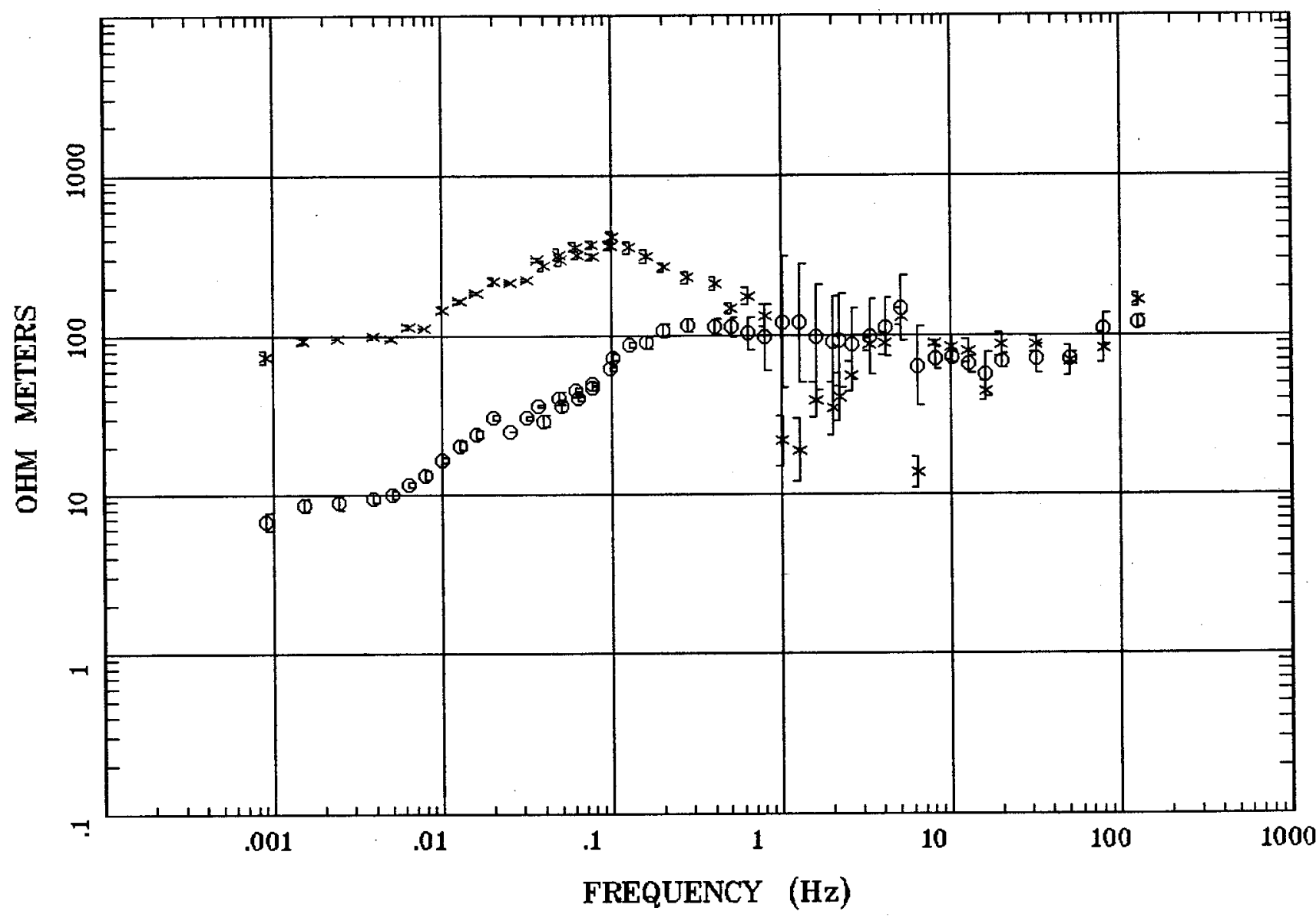

Client:

Remote:

Acquired: 19:5 Mar 08, 1998

Survey Co:
Rotation:

Filename: s2nr.a01

Channels: Ch1 Ch2 Ch3 Ch4 Ch5 Ch3 Ch4

Plotted: 11:03 Dec 07, 2004

< EMI - ElectroMagnetic Instruments > 


\section{IMPEDANCE PHASE}

Station 2

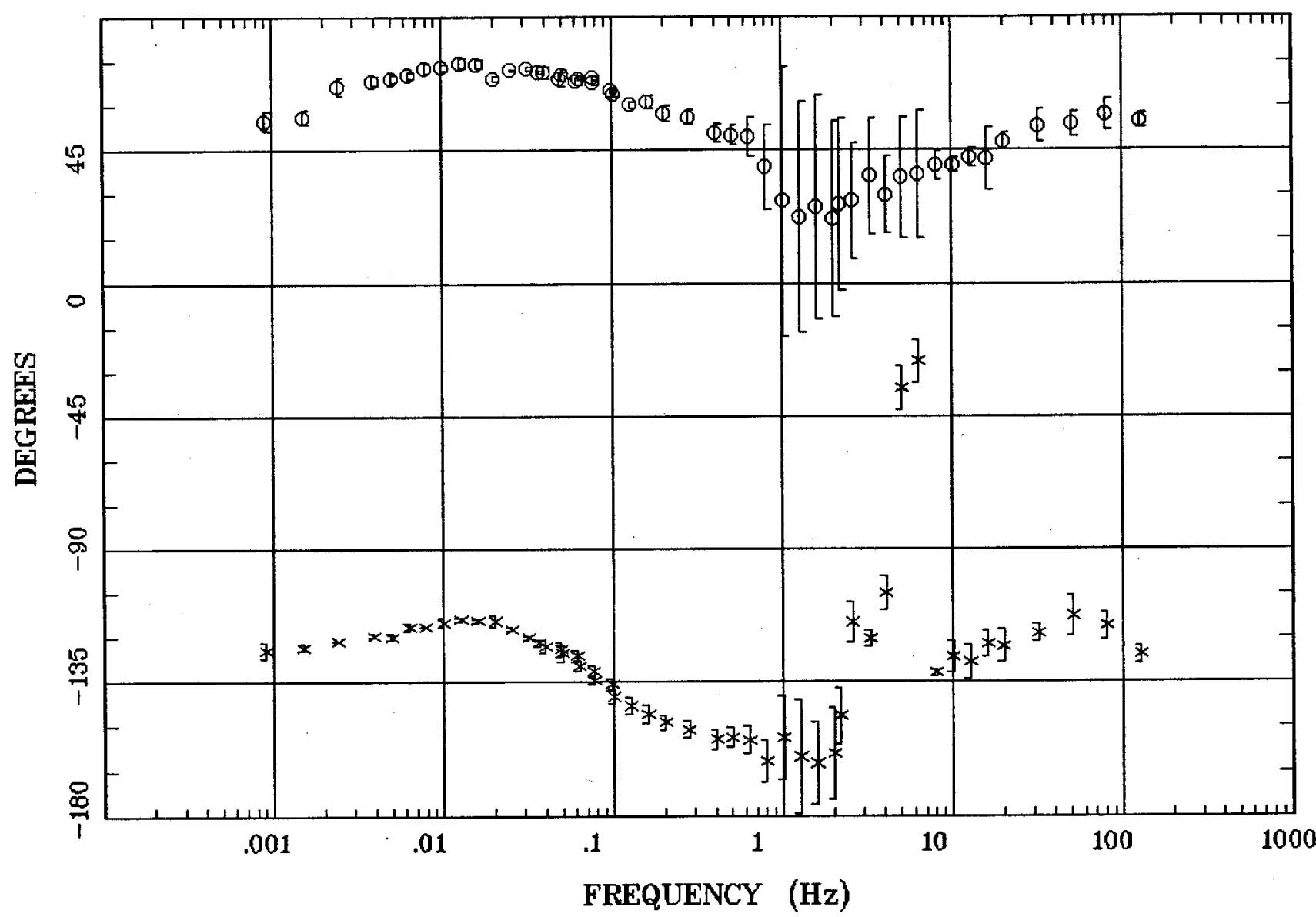

Client:

Remote:

Acquired: 19:5 Mar 08, 1998

Survey Ca:
Rotation:

Filename: s2nr.a01

Channels: Ch1 Ch2 Ch3 Ch4 Ch5 Ch3 Ch4

Plotted: 11:03 Dec 07, 2004

$<$ EMI - ElectroMagnetic Instruments > 
ROTATION ANGLE

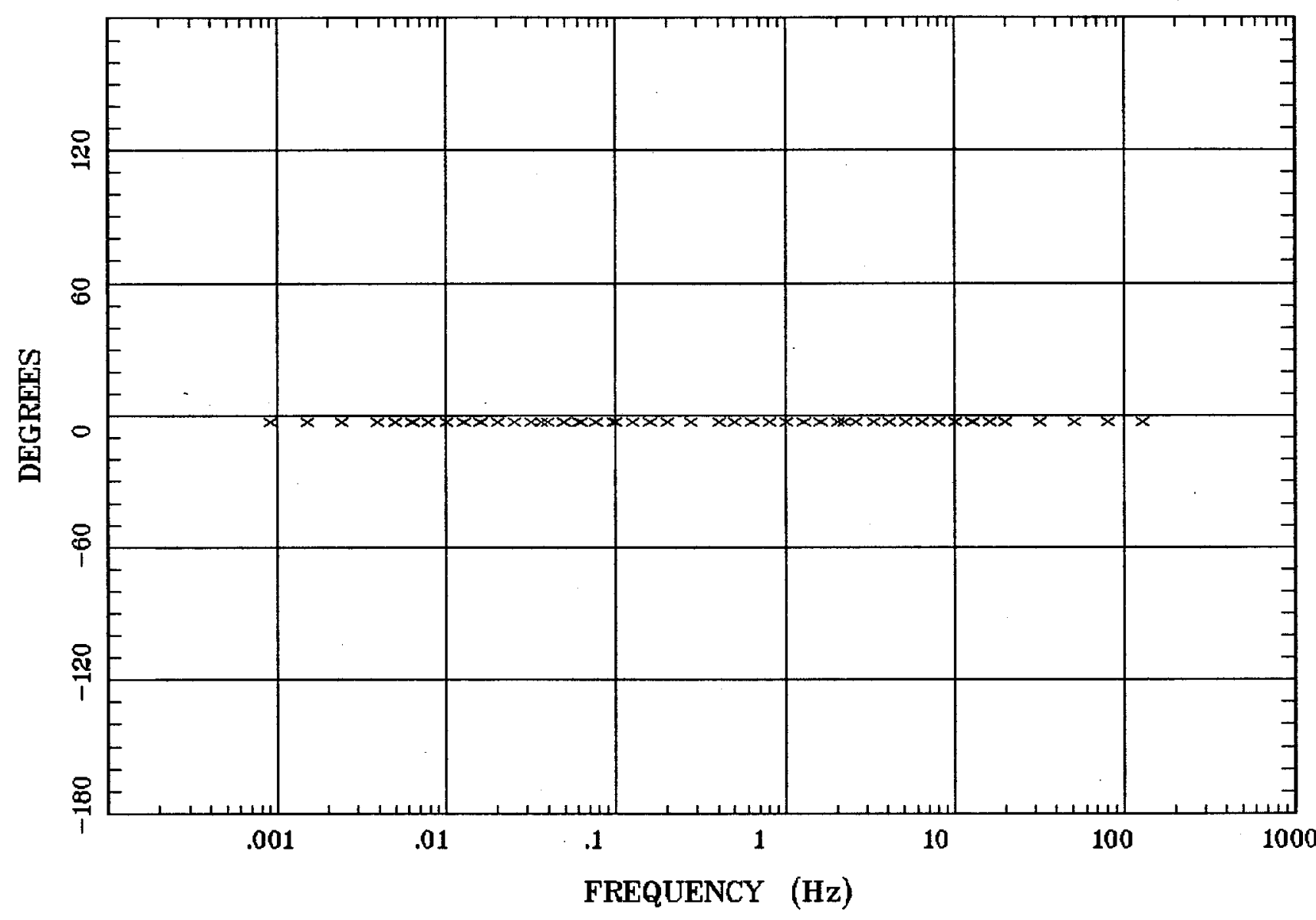

Client:

Remote:

Acquired: 19:5 Mar 08, 1998 Survey Co:
Rotation:

Filename: s2nr.a01

Channels: Ch1 Ch2 Ch3 Ch4 Ch5 Ch3 Ch4 Plotted: 11:03 Dec 07, 2004

< EMI - ElectroMagnetic Instruments 


\section{IMPEDANCE SKEW}

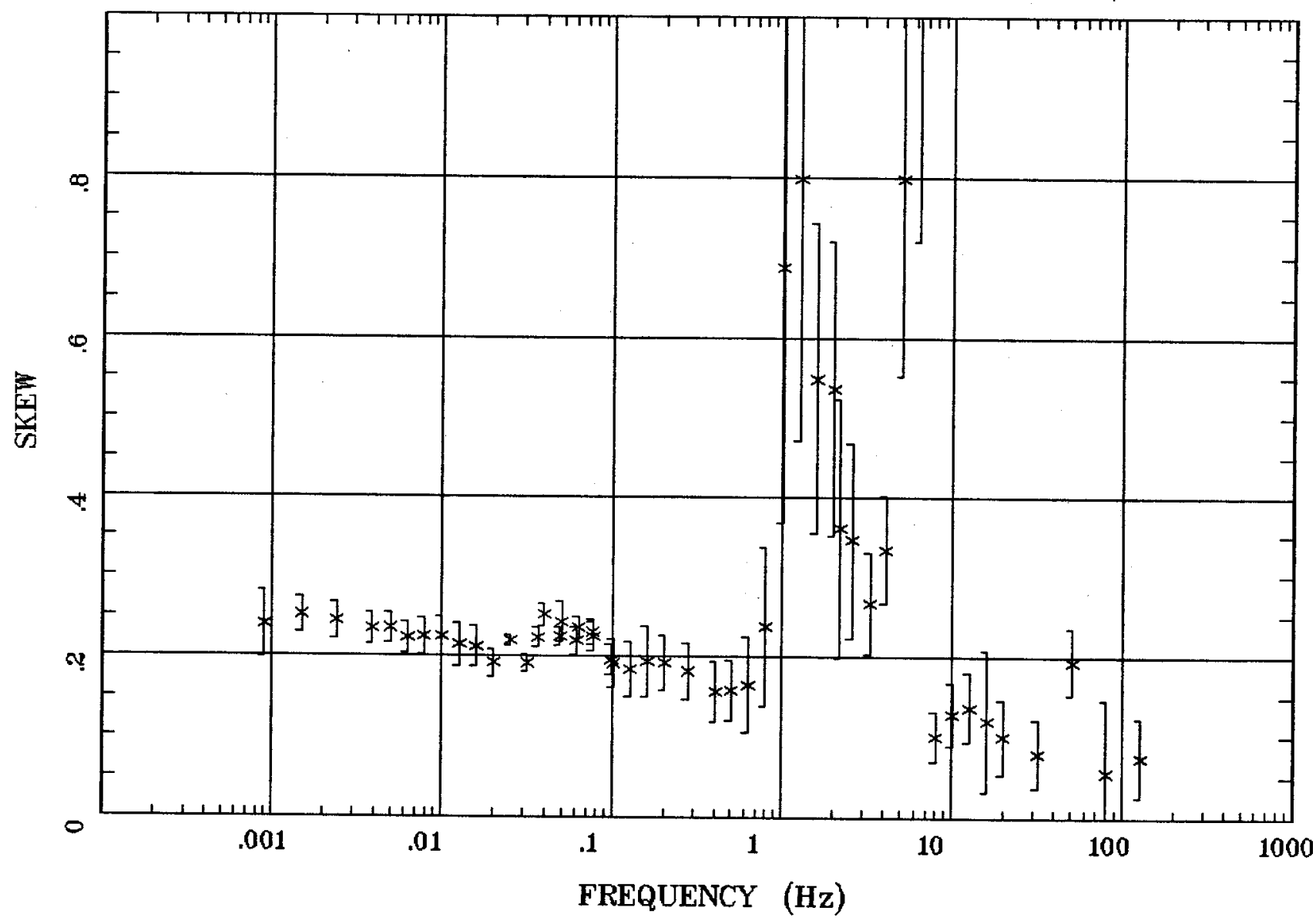

Client:

Remote:

Acquired: 19:5 Mar 08, 1998 Survey Co:

\section{Rotation:}

Filename: s2nr.a01

Channels: Ch1 Ch2 Ch3 Ch4 Ch5 Ch3 Ch4

Platted: 11:03 Dec 07, 2004

< EMI - ElectroMagnetic Instruments > 


\section{E MULT Coh.}

Station 2

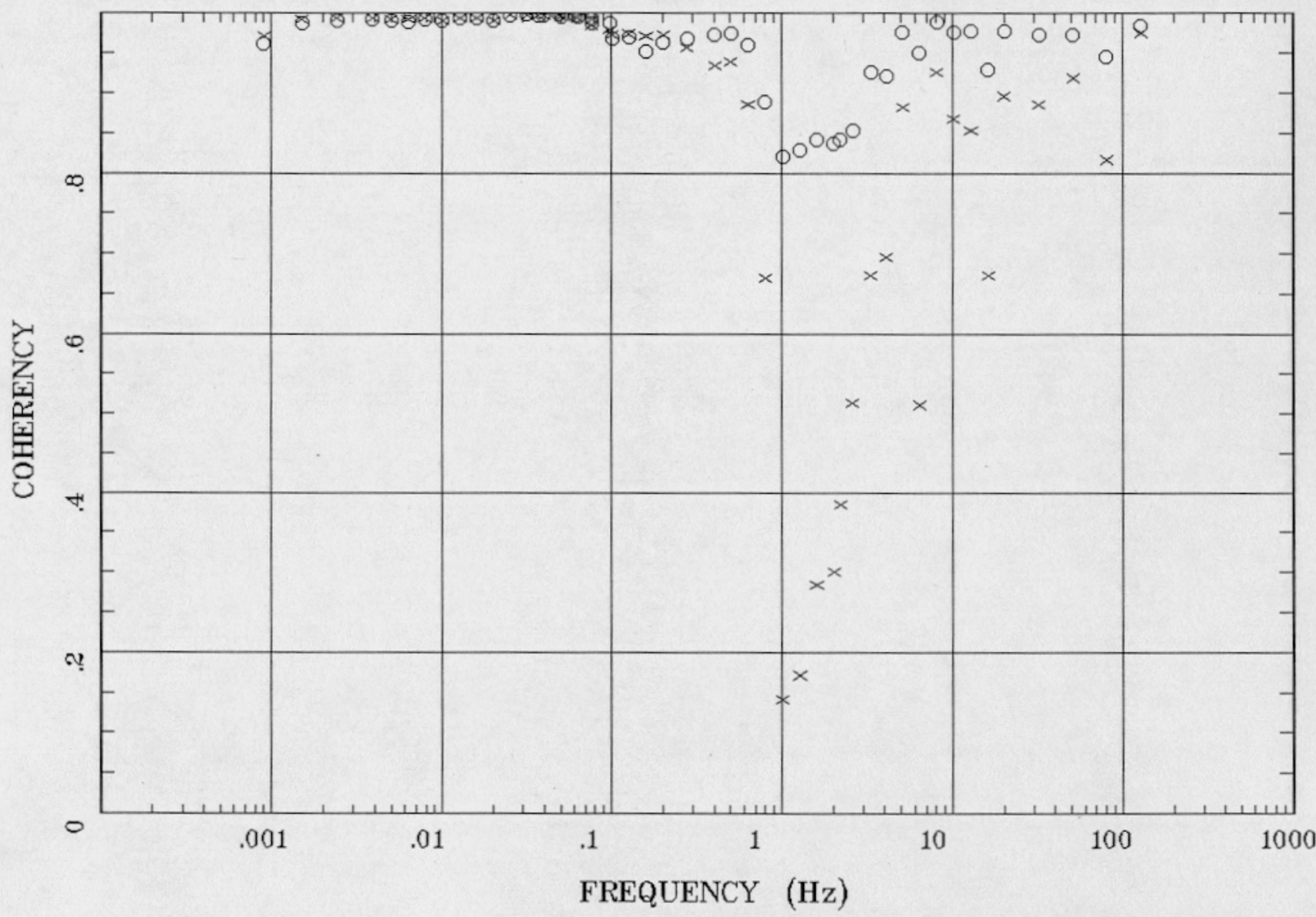

Client:

Remote:

Acquired: 19:5 Mar 08, 1998

Survey Co:
Rotation:

Filename: s2nr.a01

Channels: Ch1 Ch2 Ch3 Ch4 Ch5 Ch3 Ch4

Plotted: 11:03 Dec 07, 2004

< EMI - ElectroMagnetic Instruments > 


\section{POLAR PLOTS}

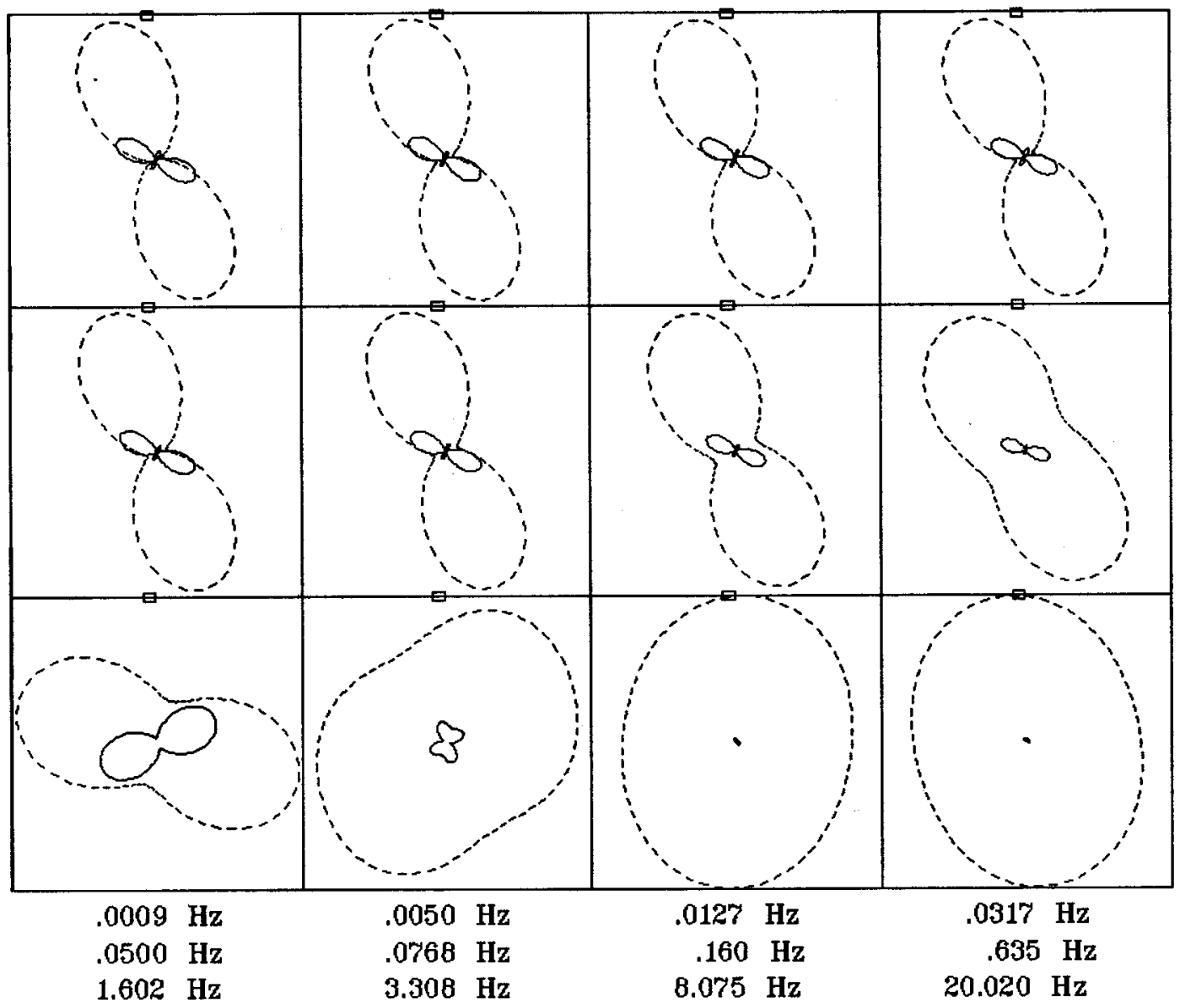

Client:

Remote:

Acquired: 19:5 Mar 08, 1998 Survey Co:

\section{Rotation:}

Filename: s2nr.a01

Channels: Ch1 Ch2 Ch3 Ch4 Ch5 Ch3 Ch4 Plotted: 11:03 Dec 07, 2004

$<$ EMI - ElectroMagnetic Instruments 


\section{TIPPER MAGNITUDE}

Station 2

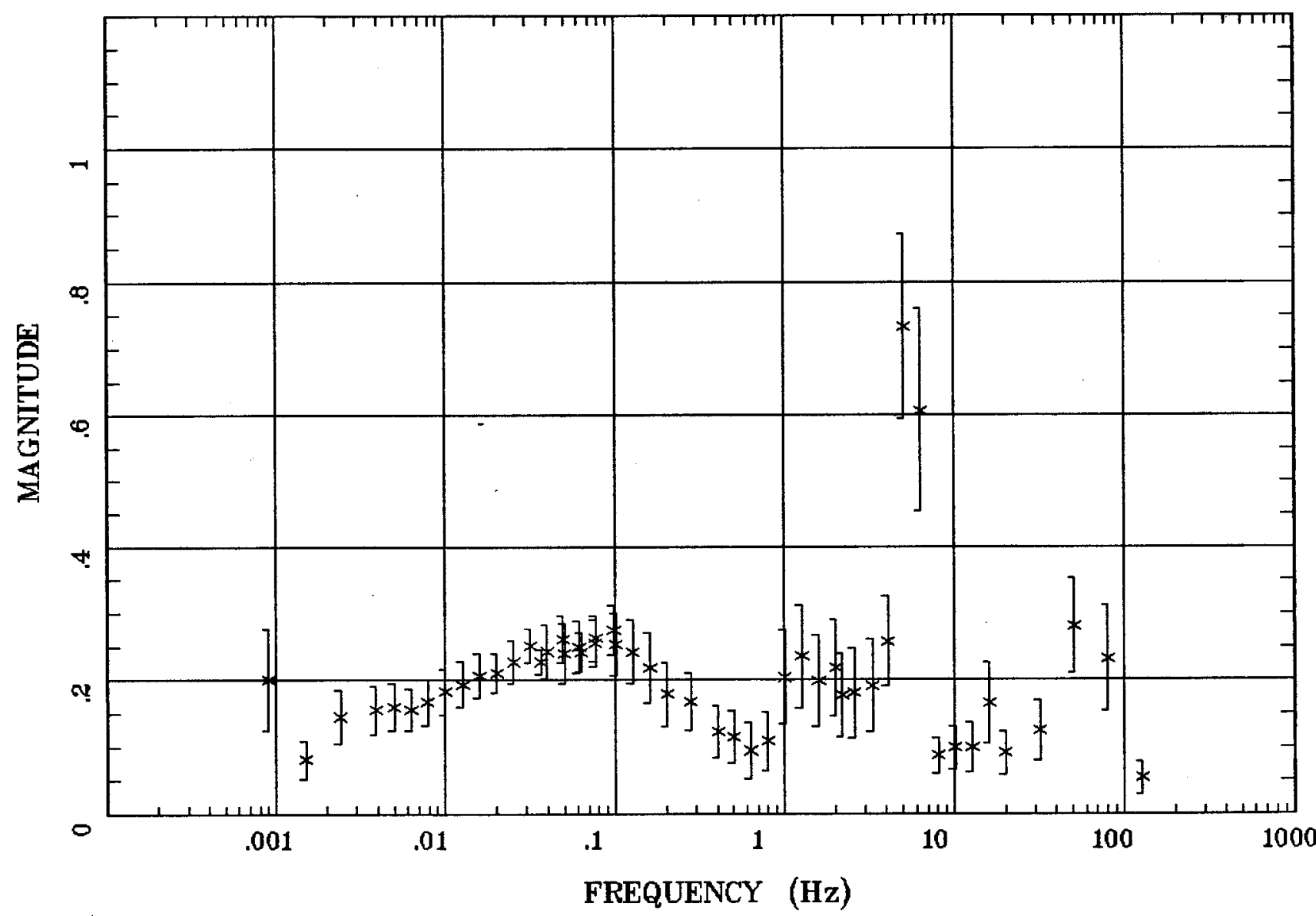

Client:

Remote:

Acquired: 19:5 Mar 08, 1998

Survey Co:
Rotation:

Filename: s2nr.a01

Channels: Ch1 Ch2 Ch3 Ch4 Ch5 Ch3 Ch4

Plotted: 11:03 Dec 07, 2004

< EMI - ElectraMagnetic Instruments 


\section{TIPPER STRIKE}

Station 2

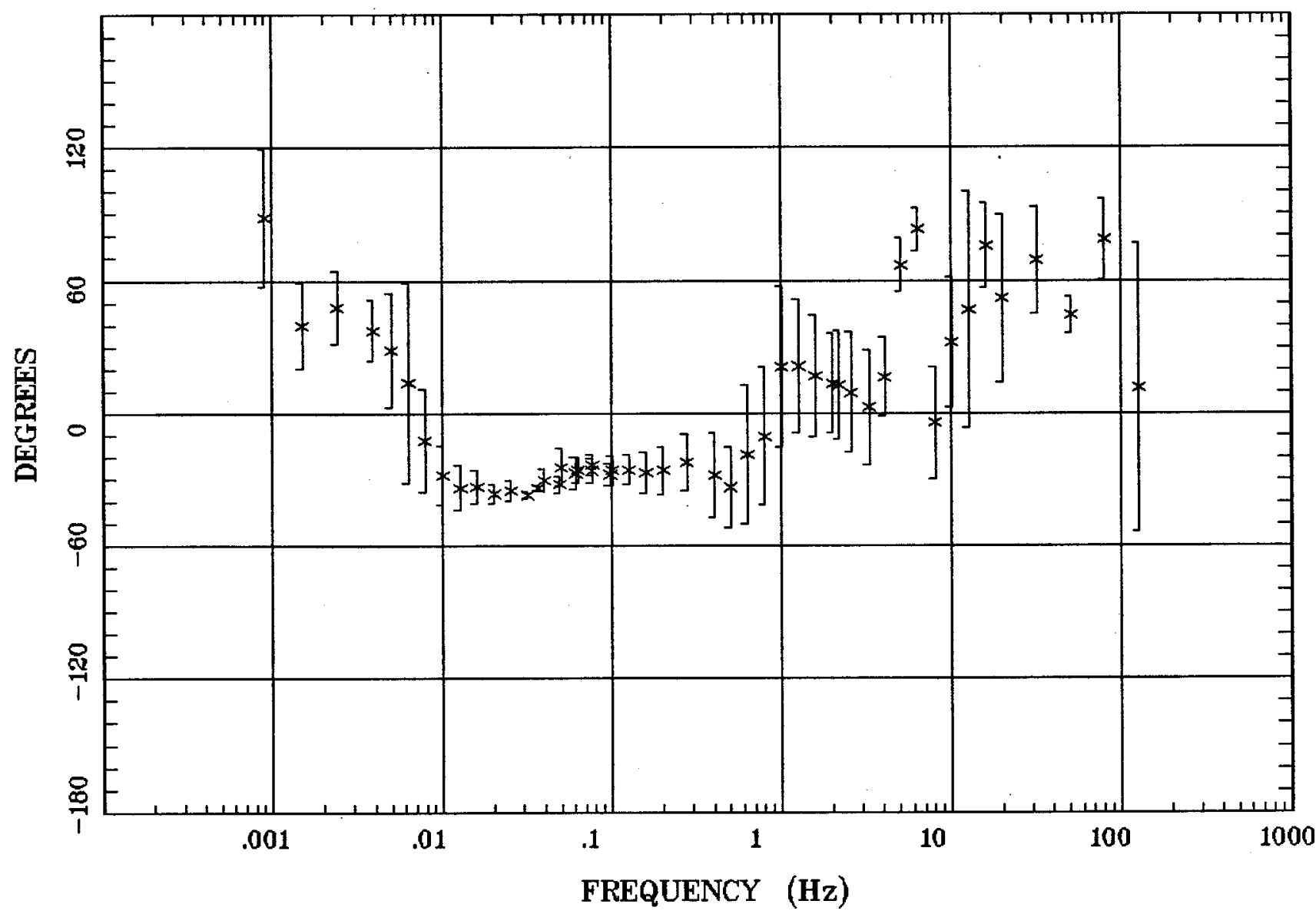

Client:

Remote:

Acquired: 19:5 Mar 08, 1998 Survey Co:
Rotation:

Filename: s2nr.a01

Channels: Ch1 Ch2 Ch3 Ch4 Ch5 Ch3 Ch4

Plotted: 11:03 Dec 07, 2004

< EMI - ElectroMagnetic Instruments 


\section{HzHx.x Coh HzHy.o}

Station 2

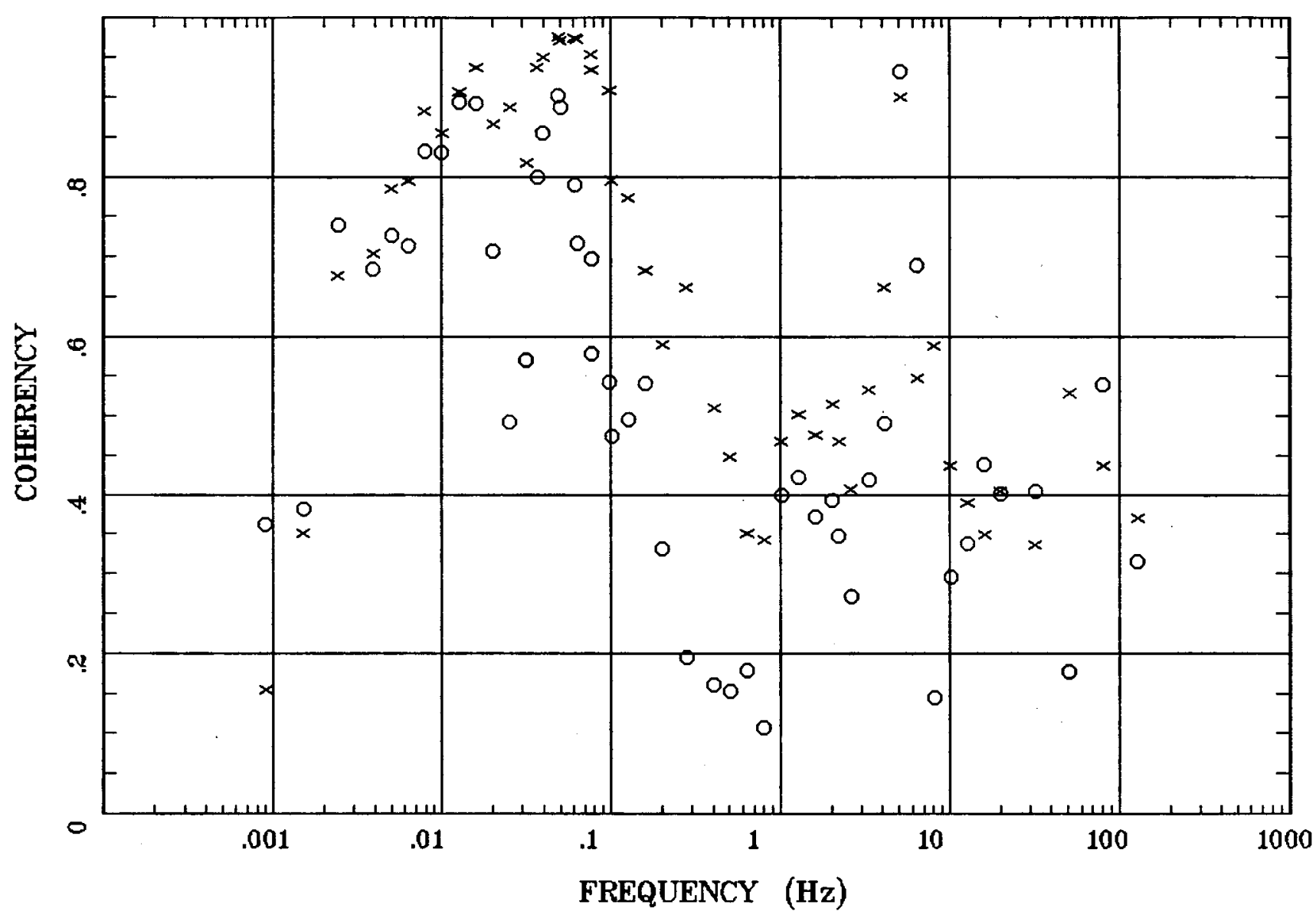

Client:

Remote:

Acquired: 19:5 Mar 08, 1998

Survey Co:
Rotation:

Filename: s2nr.a01

Channels: Ch1 Ch2 Ch3 Ch4 Ch5 Ch3 Ch4

Plotted: 11:03 Dec 07, 2004

< EMI - ElectroMagnetic Instruments > 


\section{APPARENT RESISTIVITY}

Station 3

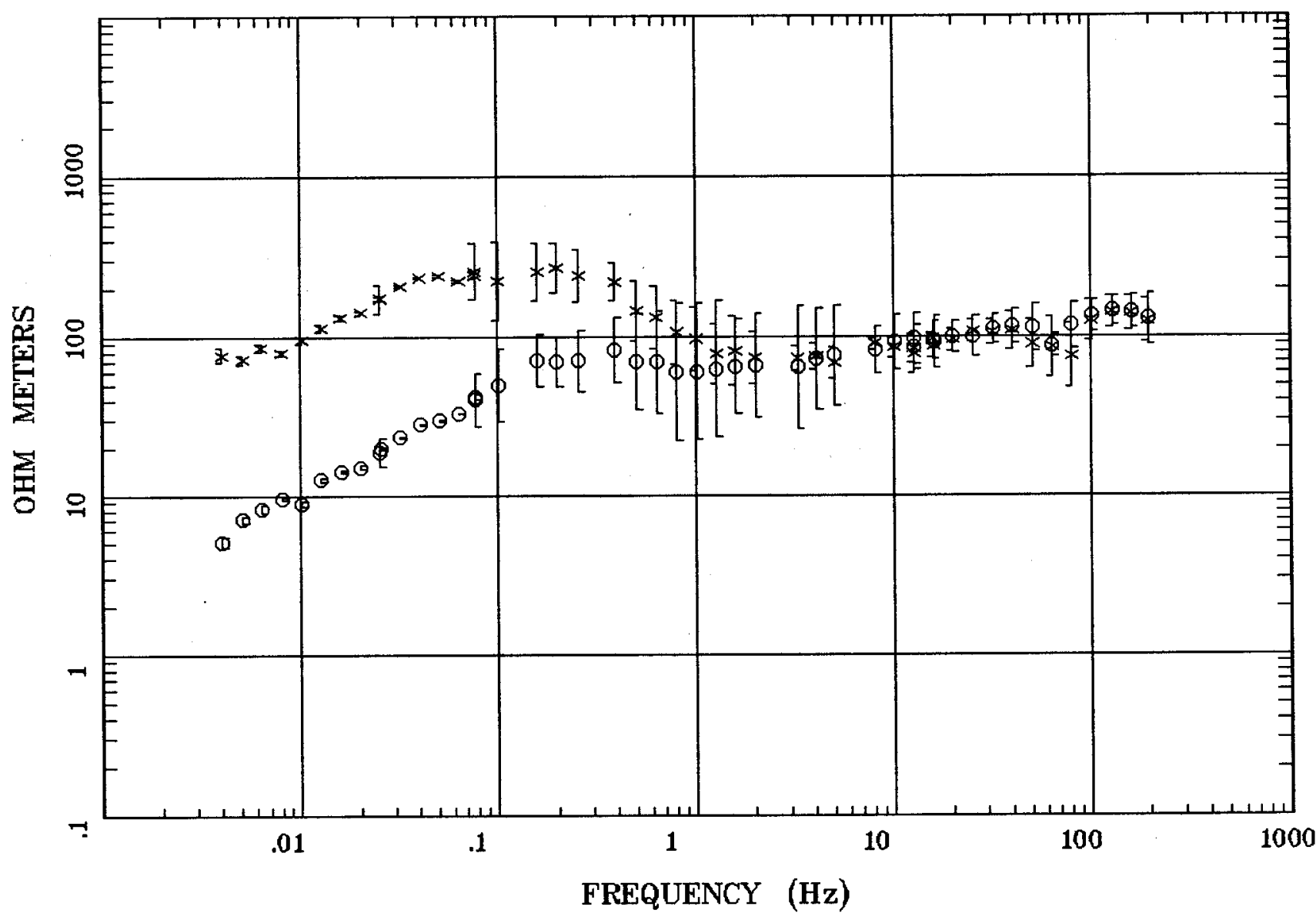

Client:

Remote:

Acquired: 19:5 Mar 08, 1998

Survey Co:
Rotation:

Filename: s3test.avg

Channels: Ch1 Ch2 Ch3 Ch4 Ch5 Ch3 Ch4

Plotted: 10:16 Dec 13, 2004

< EMI - ElectroMagnetic Instruments 


\section{IMPEDANCE PHASE}

Station 3

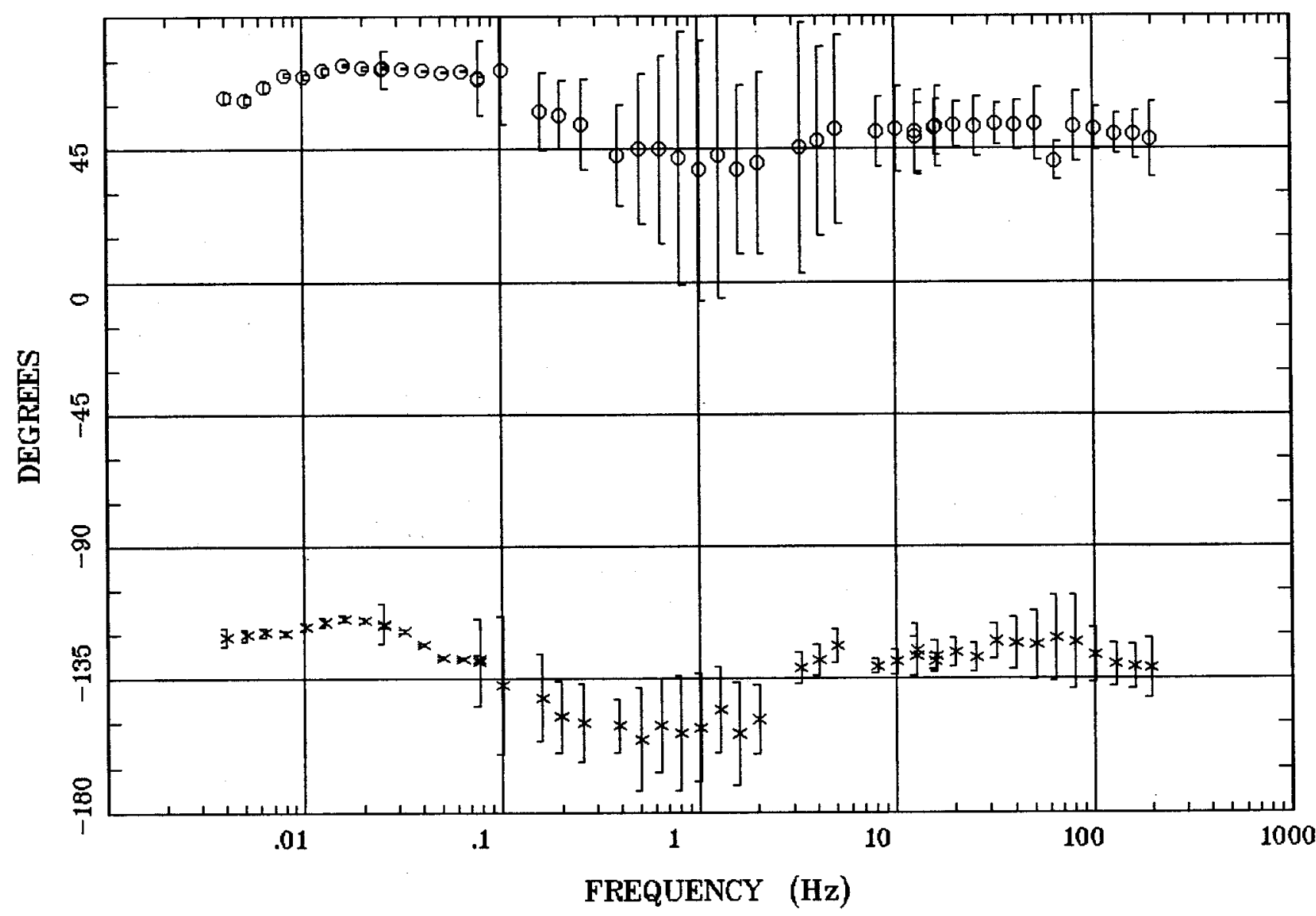

Client:

Remote:

Acquired: 19:5 Mar 08, 1998 Survey Co:
Rotation:

Filename: s3test.avg

Channels: Ch1 Ch2 Ch3 Ch4 Ch5 Ch3 Ch4 Plotted: 10:16 Dec 13, 2004

< EMI - ElectroMagnetic Instruments > 


\section{ROTATION ANGLE}

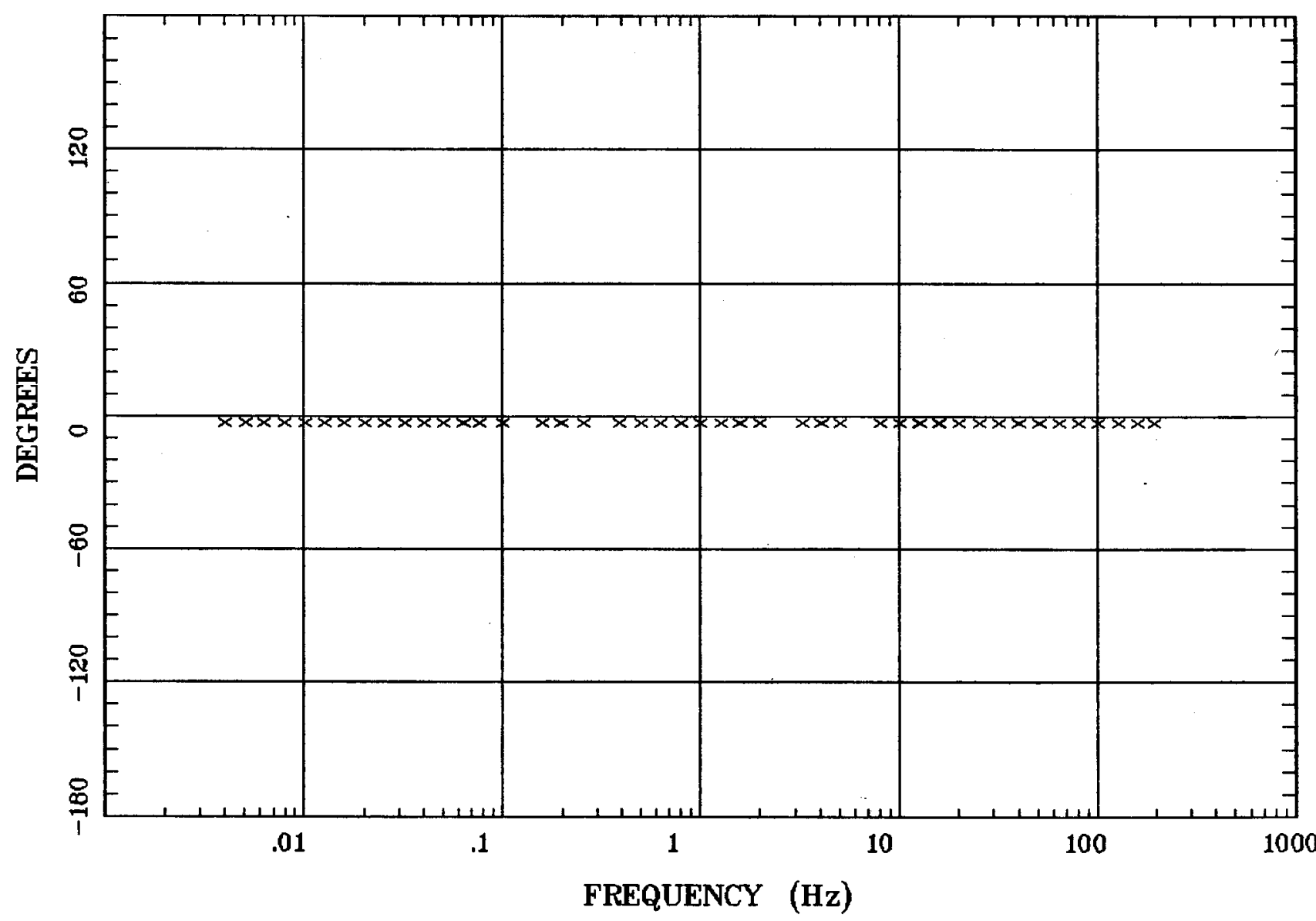

Client:

Remote:

Acquired: 19:5 Mar 08, 1998 Survey Co:
Rotation:

Filename: s3test.avg

Channels: Ch1 Ch2 Ch3 ch4 Ch5 Ch3 Ch4

Plotted: 10:16 Dec 13, 2004

$<$ EMI - ElectroMagnetic Instruments 
IMPEDANCE SKEW

Station 3

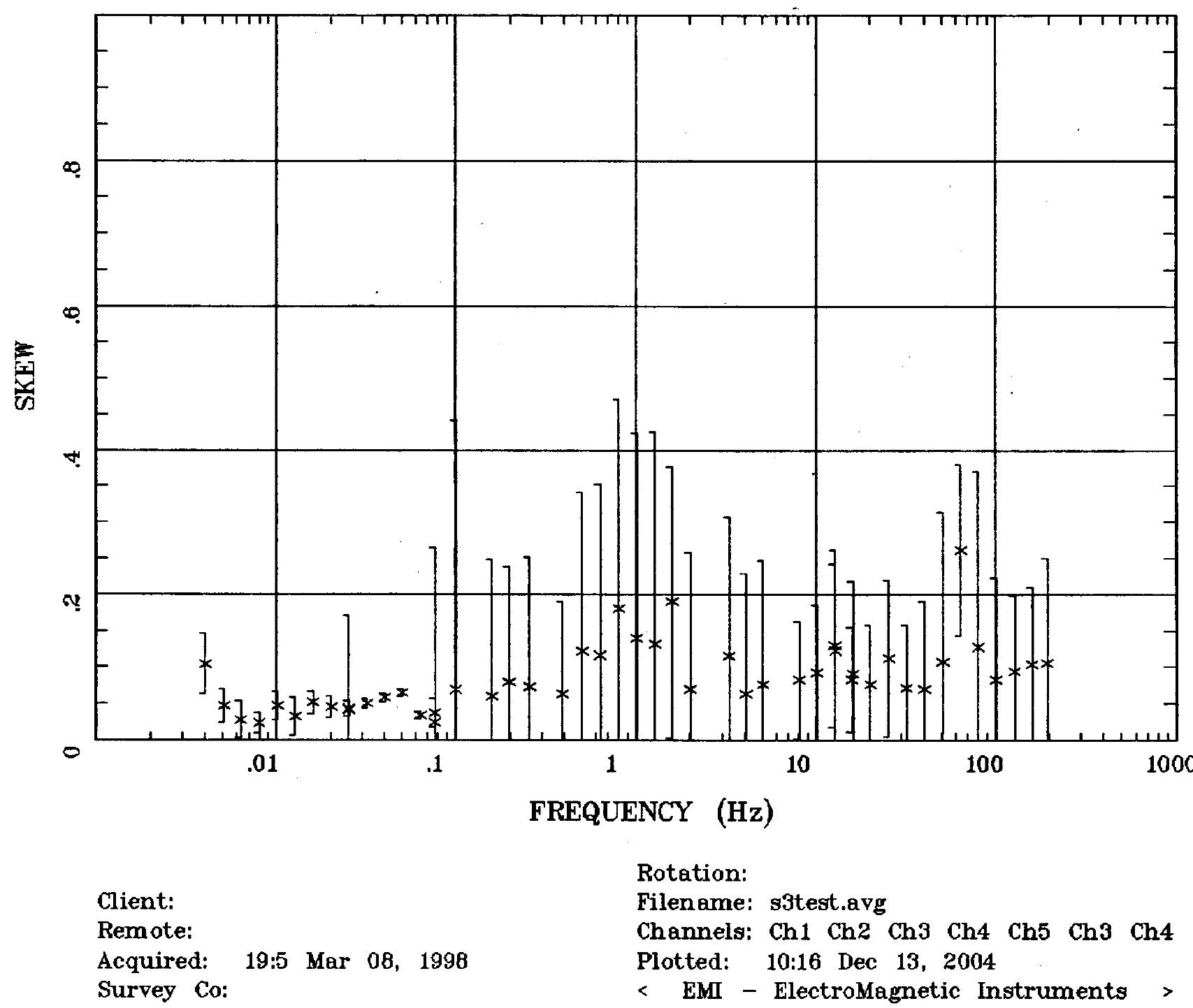




\section{E MULT Coh.}

Station 3

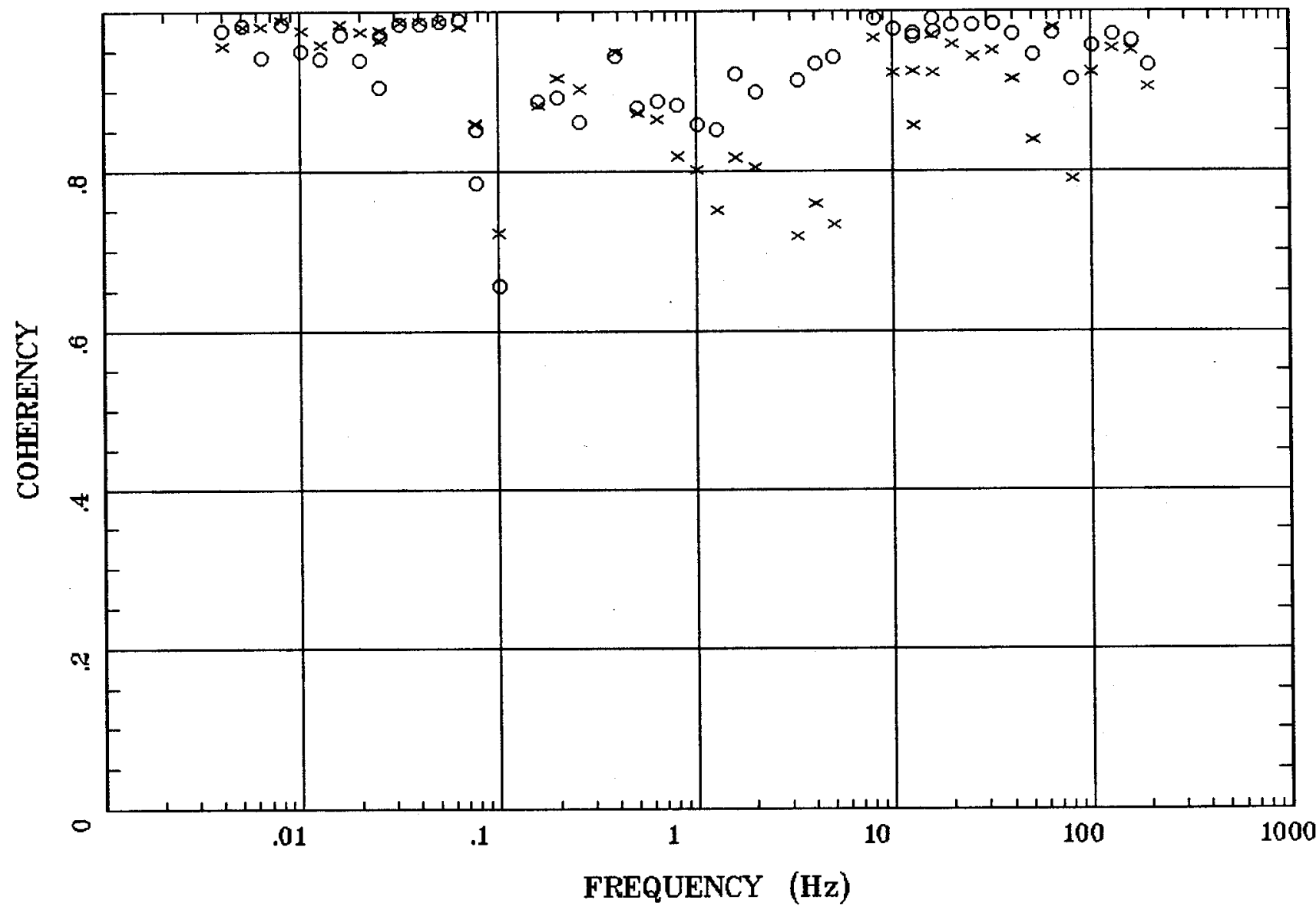

Client:

Remote:

Acquired: $19: 5$ Mar 08, 1998 Survey Co:
Rotation:

Filename: s3test.avg

Channels: Ch1 Ch2 Ch3 Ch4 Ch5 Ch3 Ch4 Plotted: 10:16 Dec 13, 2004

$<$ EMI - ElectroMagnetic Instruments 


\section{POLAR PLOTS}

Station 3

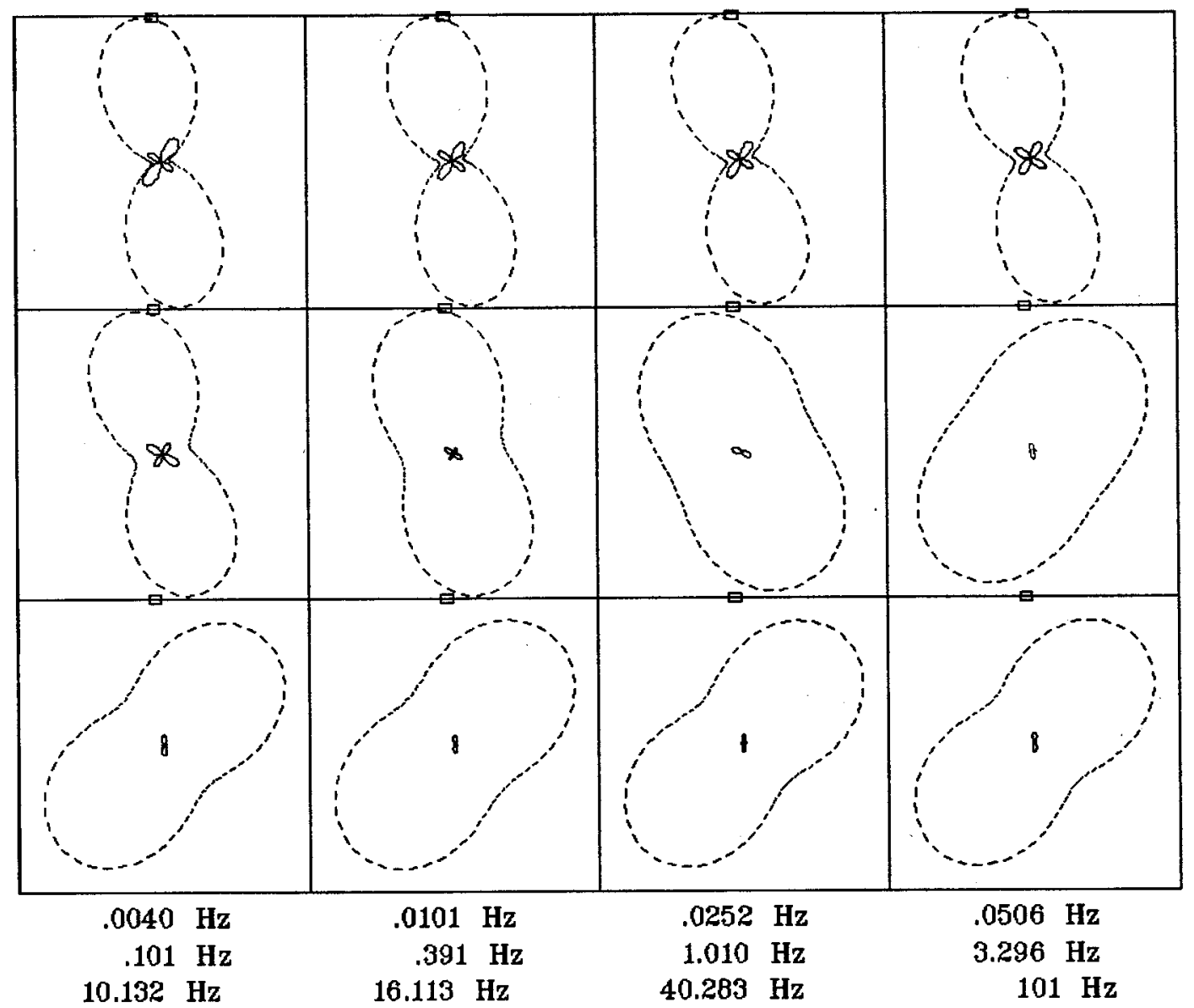

Client:

Remote:

Acquired: 19:5 Mar 08, 1998 Survey Co:
Rotation:

Filename: s3test.avg

Channels: Ch1 Ch2 Ch3 Ch4 Ch5 Ch3 Ch4 Plotted: 10:16 Dec 13, 2004

< EMI - ElectroMagnetic Instruments 
TIPPER MAGNITUDE

Station 3

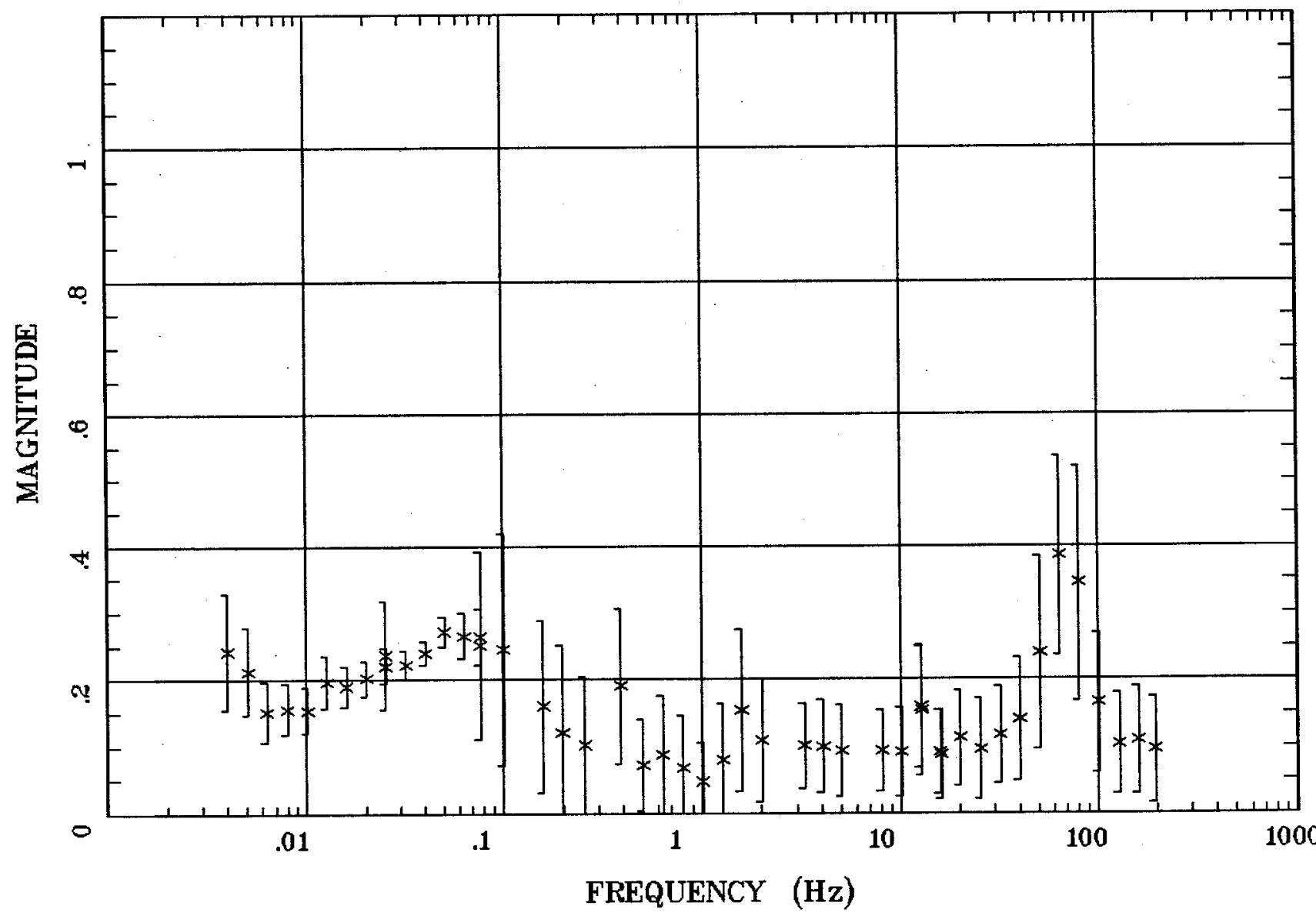

Client:

Remote:

Acquired: 19:5 Mar 08, 1998

Survey Ca:
Rotation:

Filename: s3test.avg

Channels: Ch1 Ch2 Ch3 Ch4 Ch5 Ch3 Ch4

Plotted: 10:16 Dec 13, 2004

< EMI - ElectroMagnetic Instruments > 
TIPPER STRIKE

Station 3

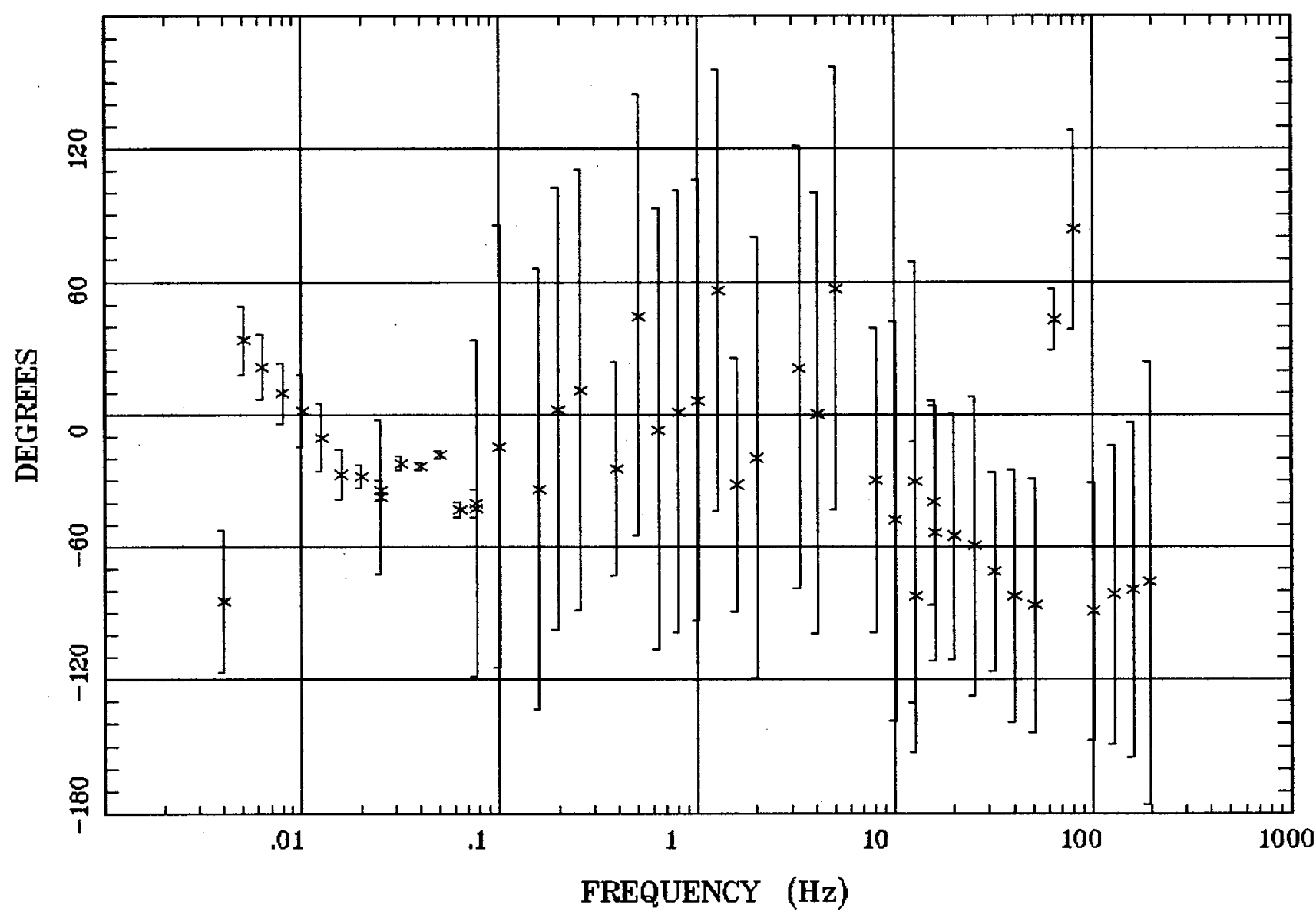

Client:

Remote:

Acquired: 19:5 Mar 08, 1998

Survey Co:
Rotation:

Filename: s3test.avg

Channels: Ch1 Ch2 Ch3 Ch4 Ch5 Ch3 Ch4

Plotted: 10:16 Dec 13, 2004

$<$ EMI - ElectroMagnetic Instruments > 
HzHx.x Coh HzHy.o

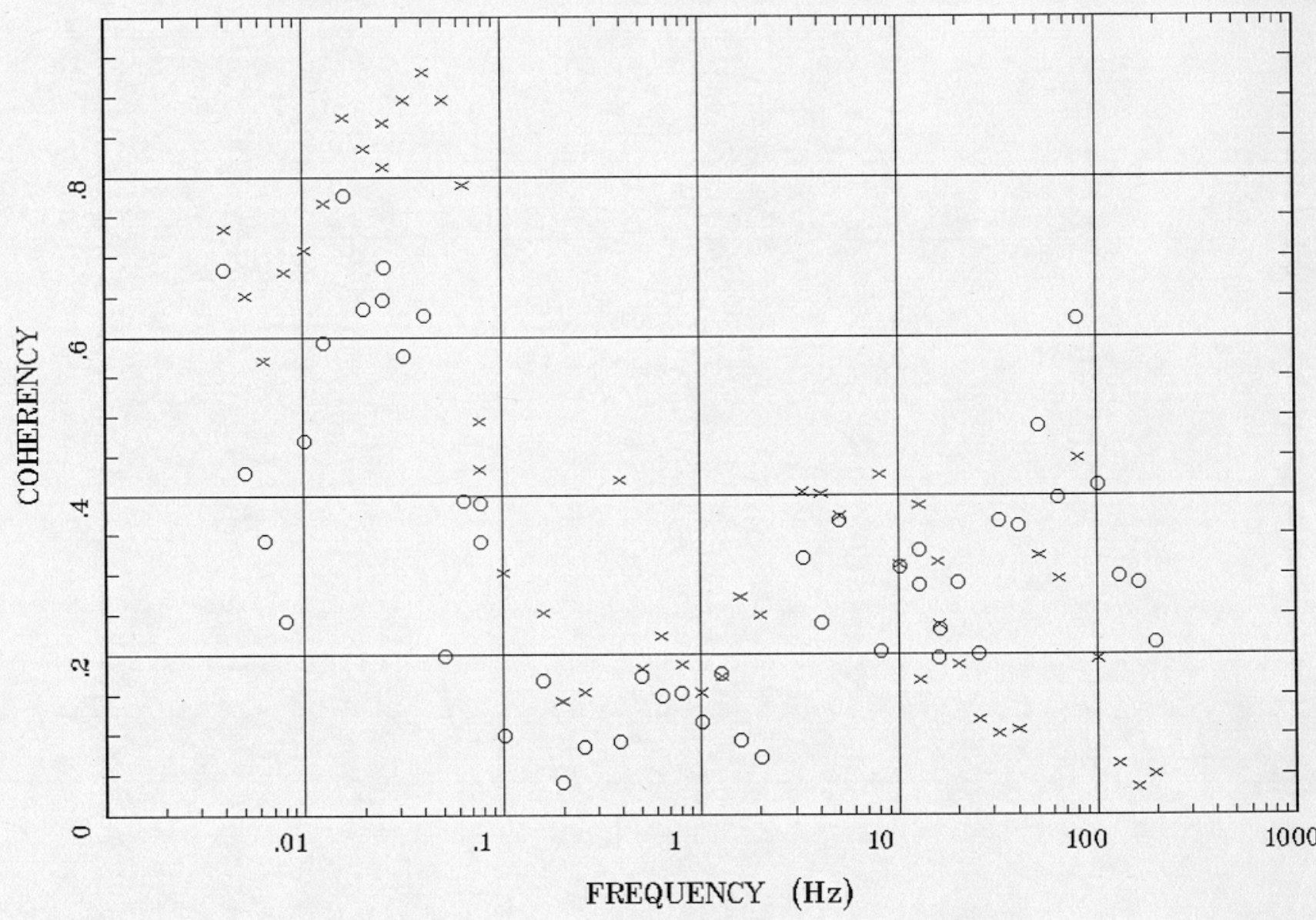

Client:

Remote:

Acquired: 19:5 Mar 08, 1998

Survey Co:
Rotation:

Filename: s3test.avg

Channels: Ch1 Ch2 Ch3 Ch4 Ch5 Ch3 Ch4

Plotted: 10:16 Dec 13, 2004

$<$ EMI - ElectroMagnetic Instruments 
APPARENT RESISTIVITY

Station 4

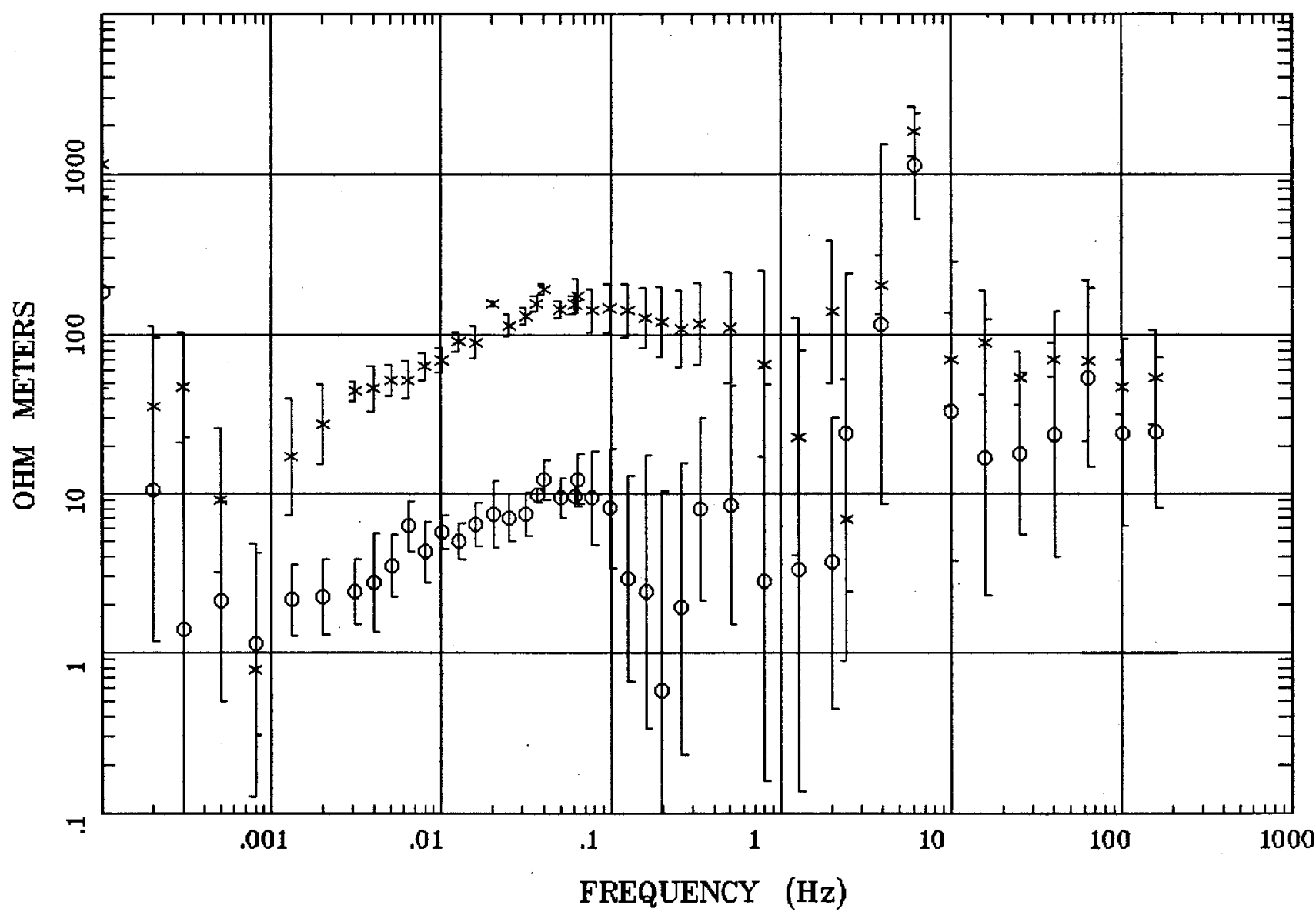

Client:

Remote:

Acquired: 19:5 Mar 08, 1998 Survey Co:
Rotation:

Filename: s4r16.a02

Channels: Ch1 Ch2 Ch3 ch4 ch5 Ch3 Ch4 Plotted: 10:58 Dec 07, 2004

< EMI - ElectroMagnetic Instruments > 


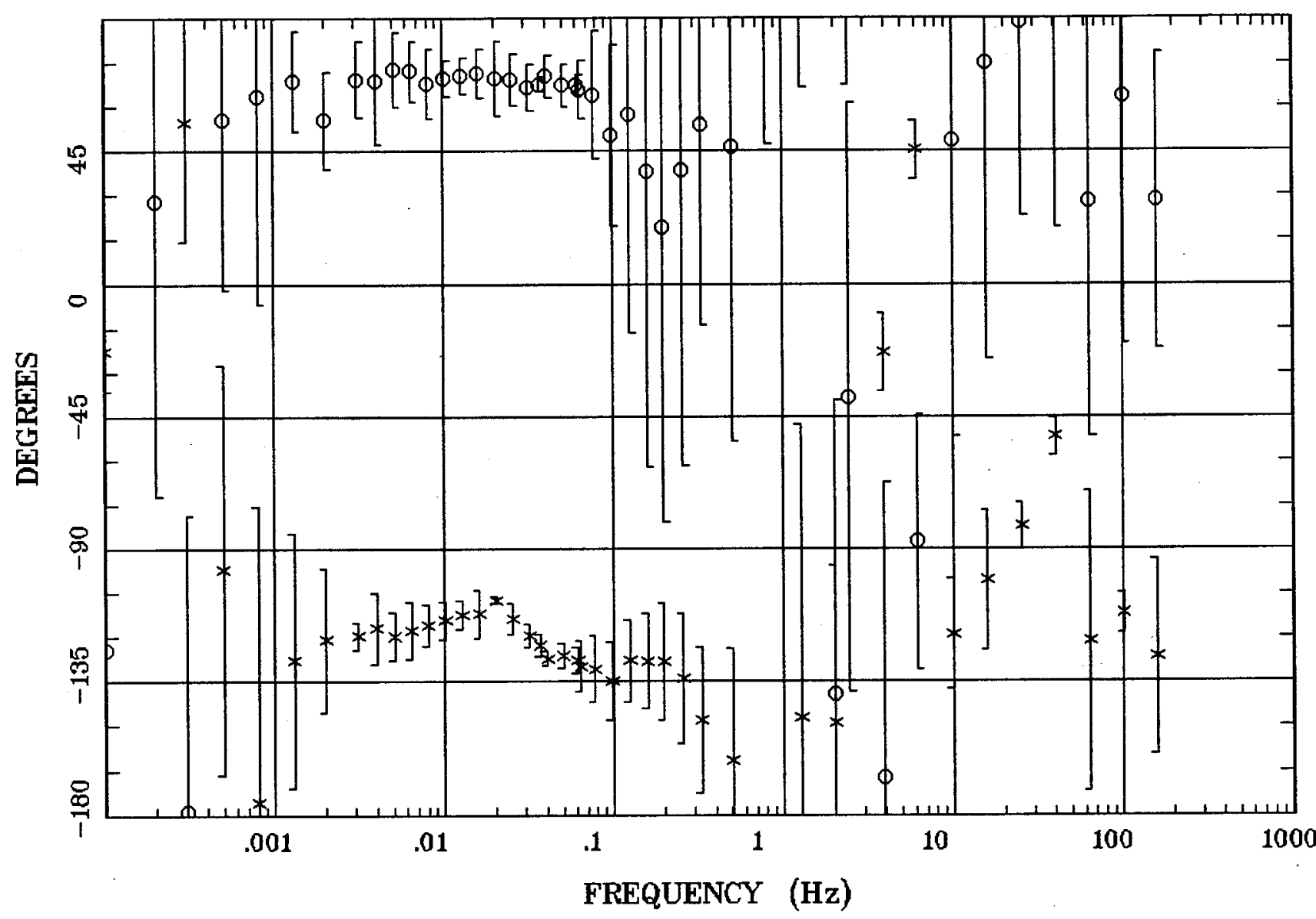

Client:

Remote:

Acquired: 19:5 Mar 08, 1998 Survey Co:
Rotation:

Filename: $54 r 16 . a 02$

Channels: Ch1 Ch2 Ch3 Ch4 Ch5 Ch3 Ch4 Plotted: 10:58 Dec 07, 2004

$<$ EMI - ElectroMagnetic Instruments > 


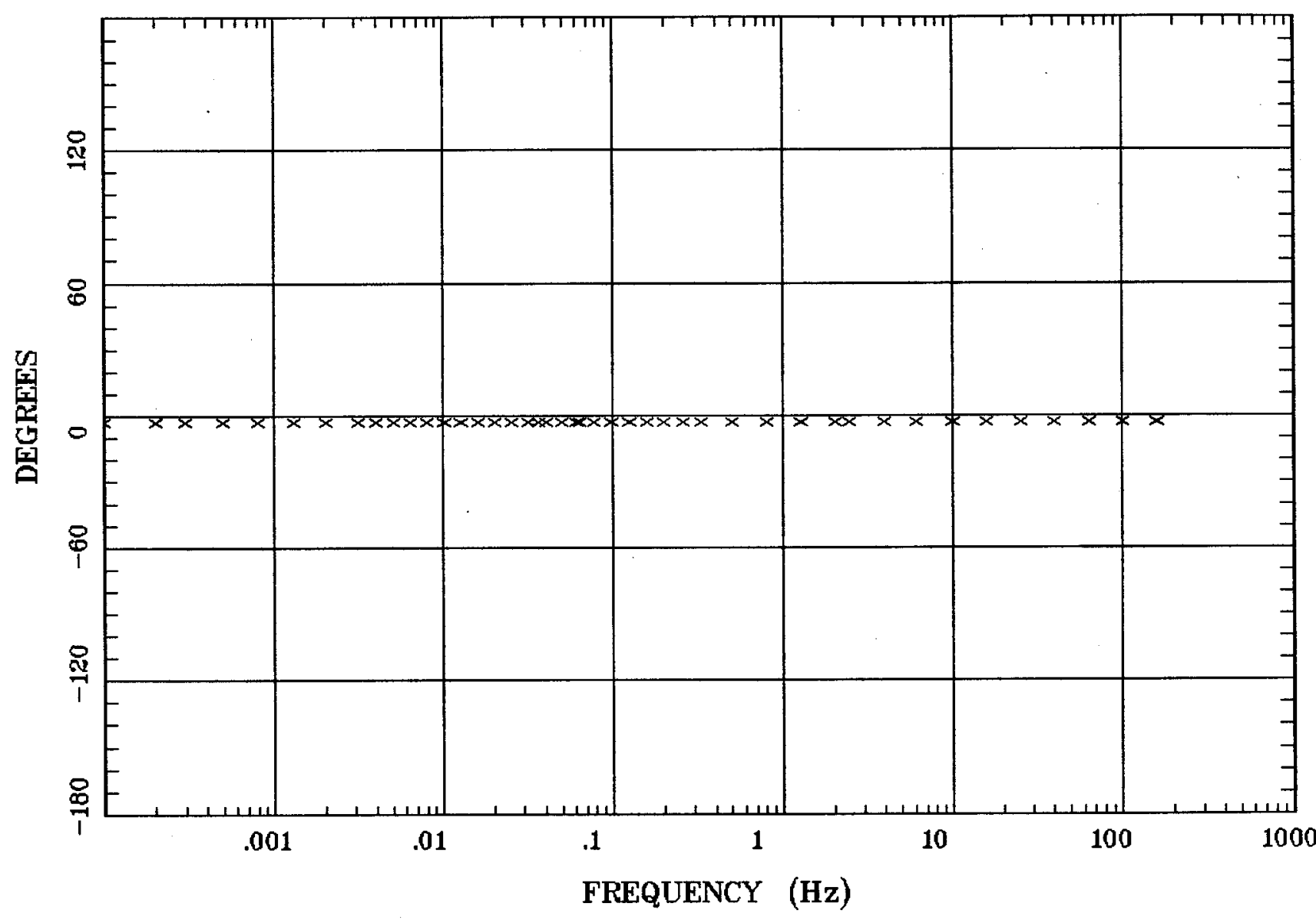

Client:

Remote:

Acquired: 19:5 Mar 08, 1998

Survey Co:
Rotation:

Filename: s4r16.e02

Channels: Ch1 Ch2 Ch3 Ch4 Ch5 Ch3 Ch4

Plotted: 10:58 Dec 0\%, 2004

$<$ EMI - ElectroMagnetic Instruments 
IMPEDANCE SKEW

Station 4

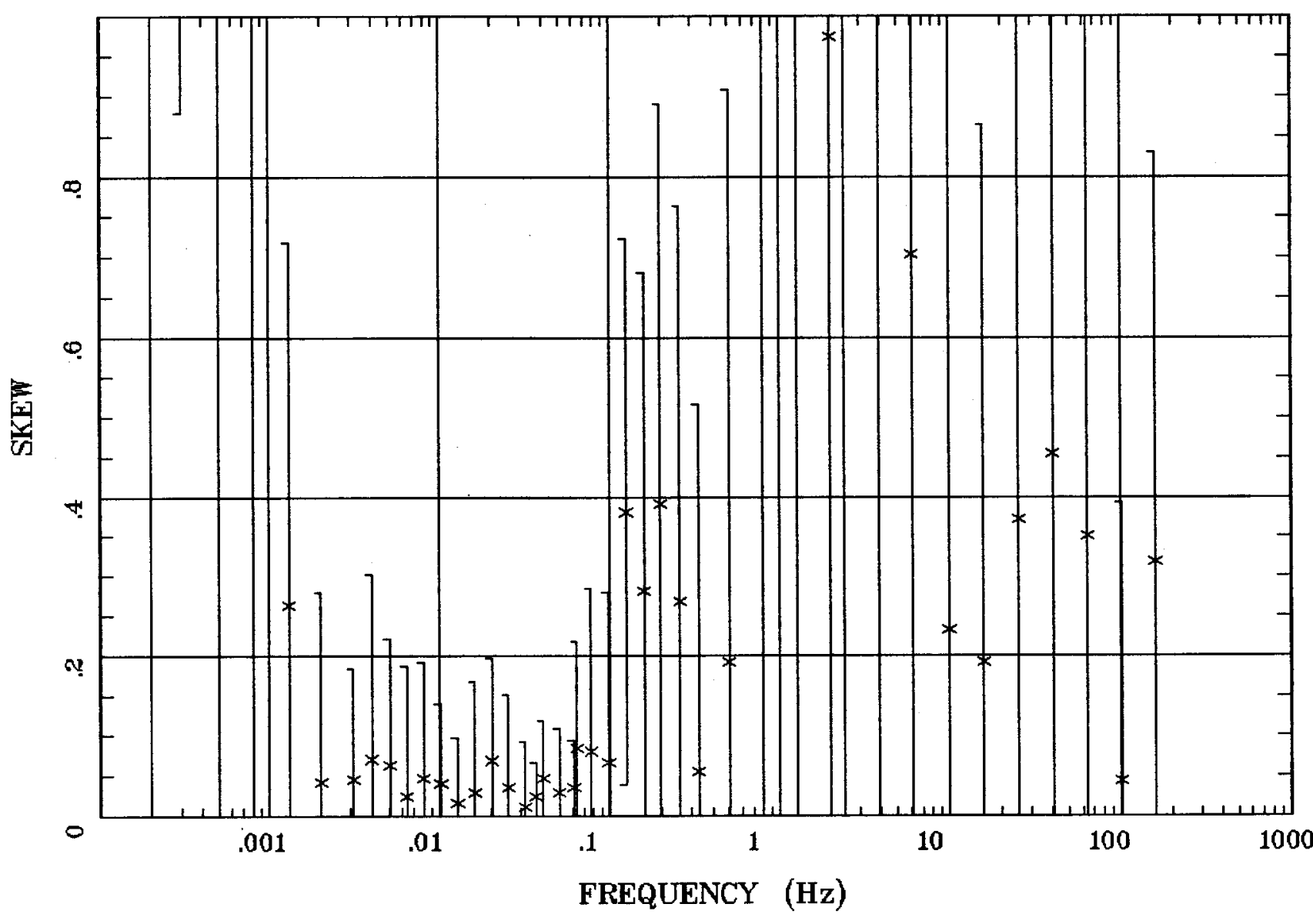

Client:

Remote:

Acquired: 19:5 Mar 08, 1998 Survey Co:
Rotation:

Filename: s4r16.a02

Channels: Ch1 Ch2 Ch3 Ch4 Ch5 Ch3 Ch4 Plotted: 10:58 Dec 07, 2004

< EMI - ElectroMagnetic Instruments > 


\section{E MULT Coh.}

\section{Station 4}

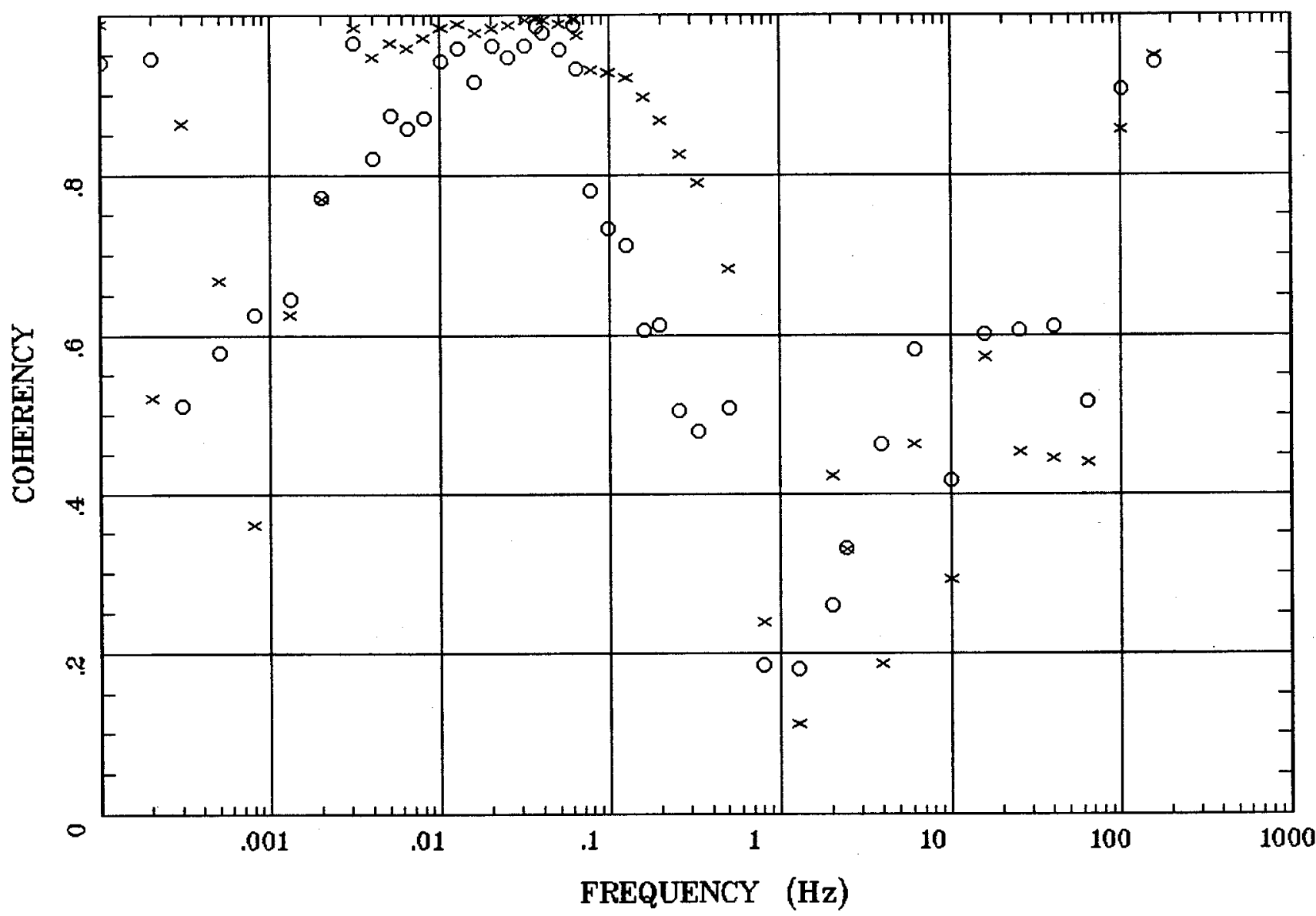

Client:

Remote:

Acquired: 19:5 Mar 08, 1998 Survey Co:
Rotation:

Filename: $s 4 r 16 . a 02$

Channels: Ch1 Ch2 Ch3 Ch4 Ch5 Ch3 Ch4 Plotted: 10:58 Dec 07, 2004

< EMI - ElectroMagnetic Instruments 


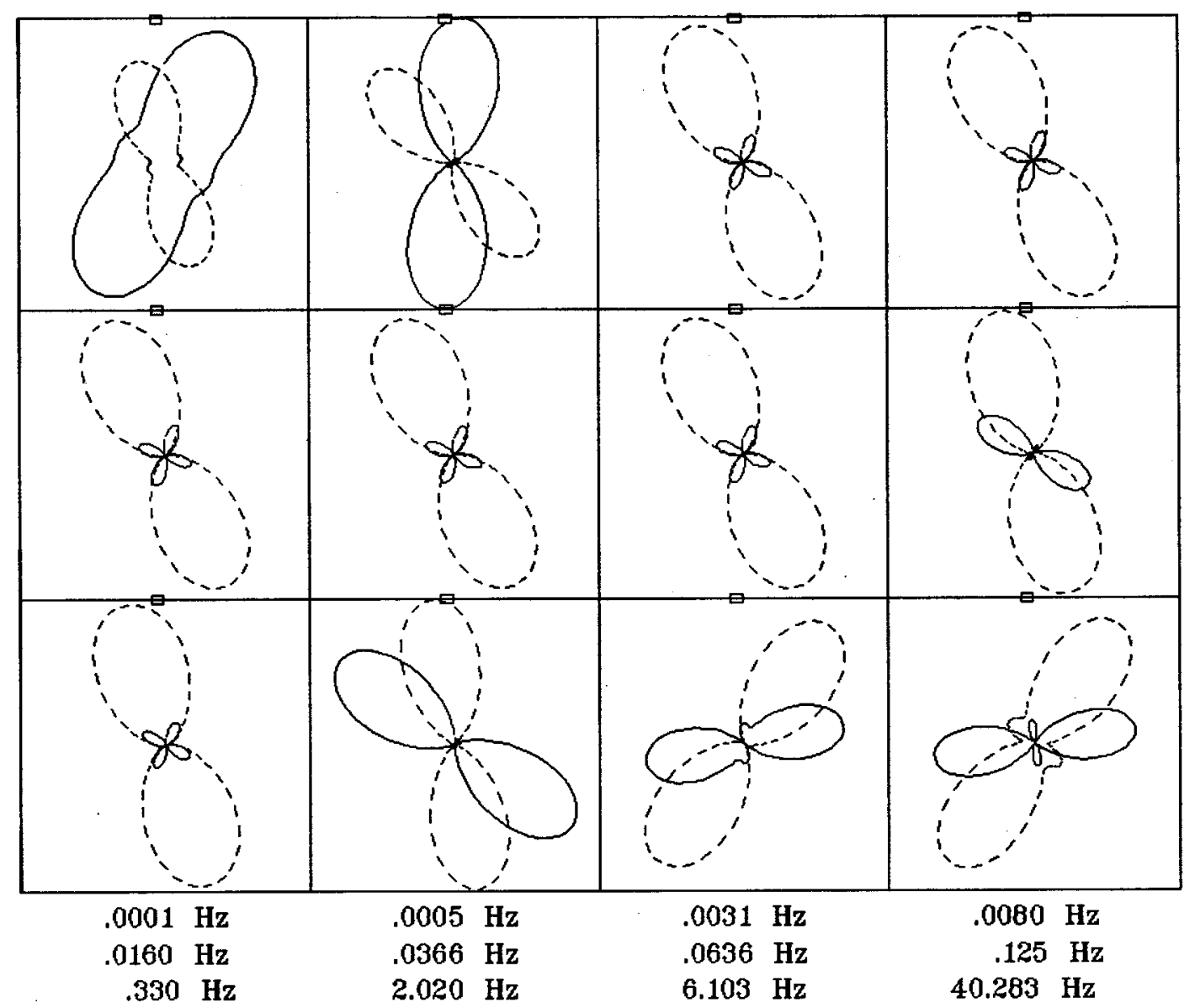

Rotation:

Client:

Remote:

Acquired: 19:5 Mar 08, 1998 Survey Co:
Filename: s4r16.a02

Channels: Ch1 Ch2 Ch3 Ch4 Ch5 Ch3 Ch4 Plotted: 10:59 Dec 07, 2004

< EMI - ElectroMagnetic Instruments 


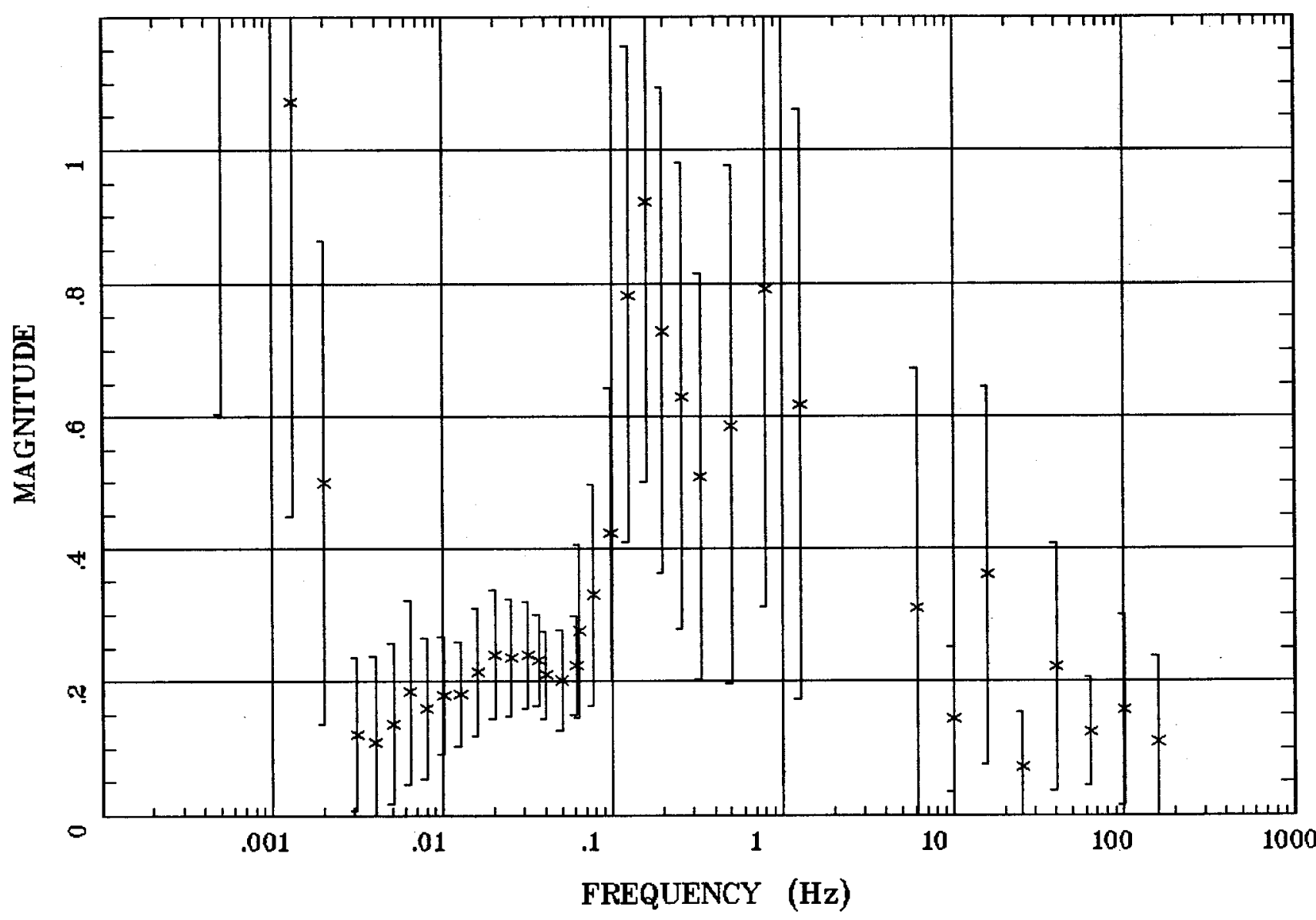

Client:

Remote:

Acquired: 19:5 Mar 08, 1998 Survey Co:
Rotation:

Filename: $\mathrm{s} 4 \mathrm{r} 16 . \mathrm{a} 02$

Channels: Ch1 Ch2 Ch3 Ch4 Ch5 Ch3 Ch4 Plotted: 10:59 Dec 07, 2004

< EMI - ElectroMagnetic Instruments 


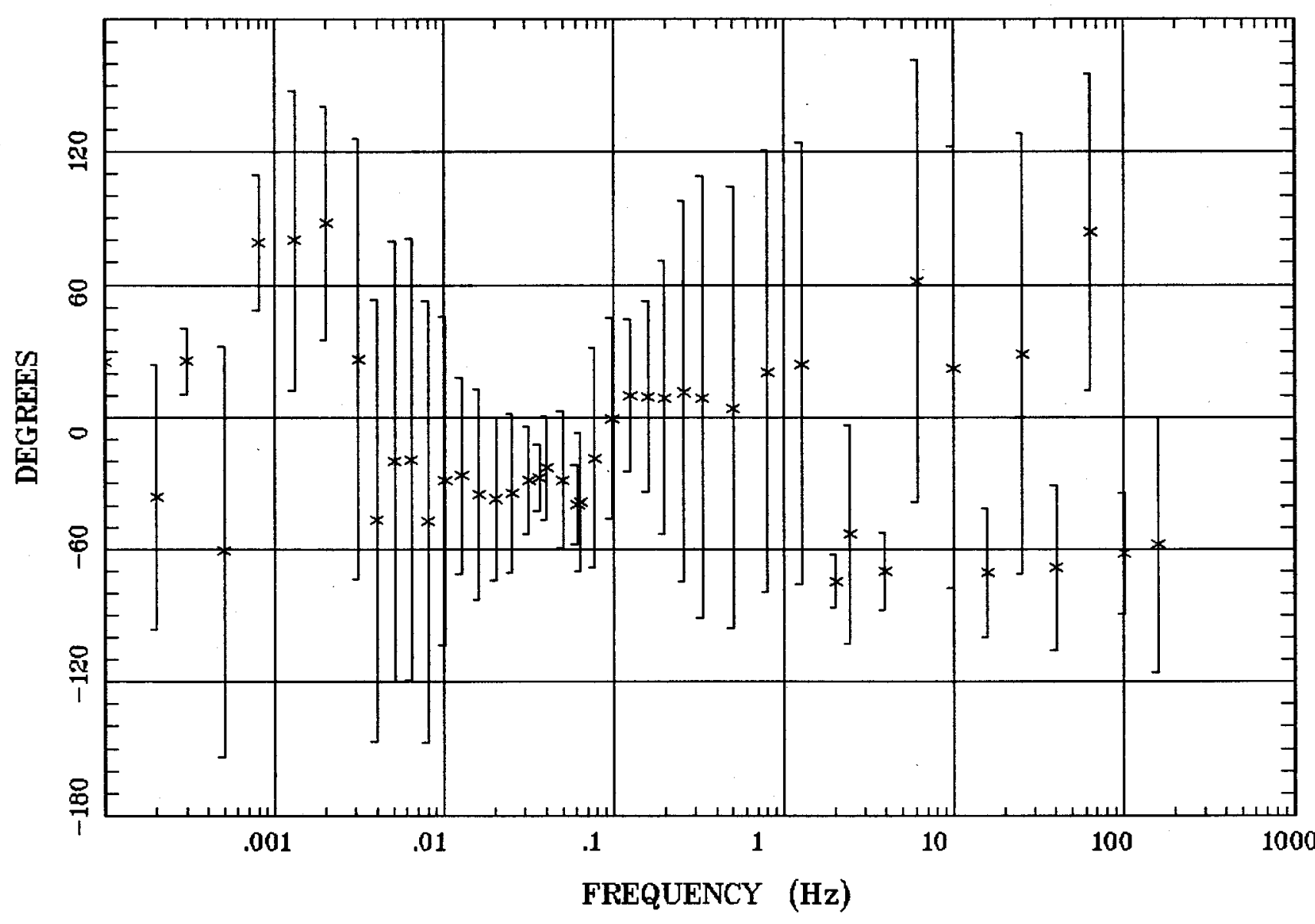

Client:

Remote:

Acquired: 19:5 Mar 08, 1998 Survey Co:
Rotation:

Filename: s4r16.a02

Channels: Ch1 Ch2 Ch3 Ch4 Ch5 Ch3 Ch4 Plotted: 10:59 Dec 07, 2004

$<$ EMI - ElectroMagnetic Instruments 


\section{HzHx.x Coh HzHy.o}

\section{Station 4}

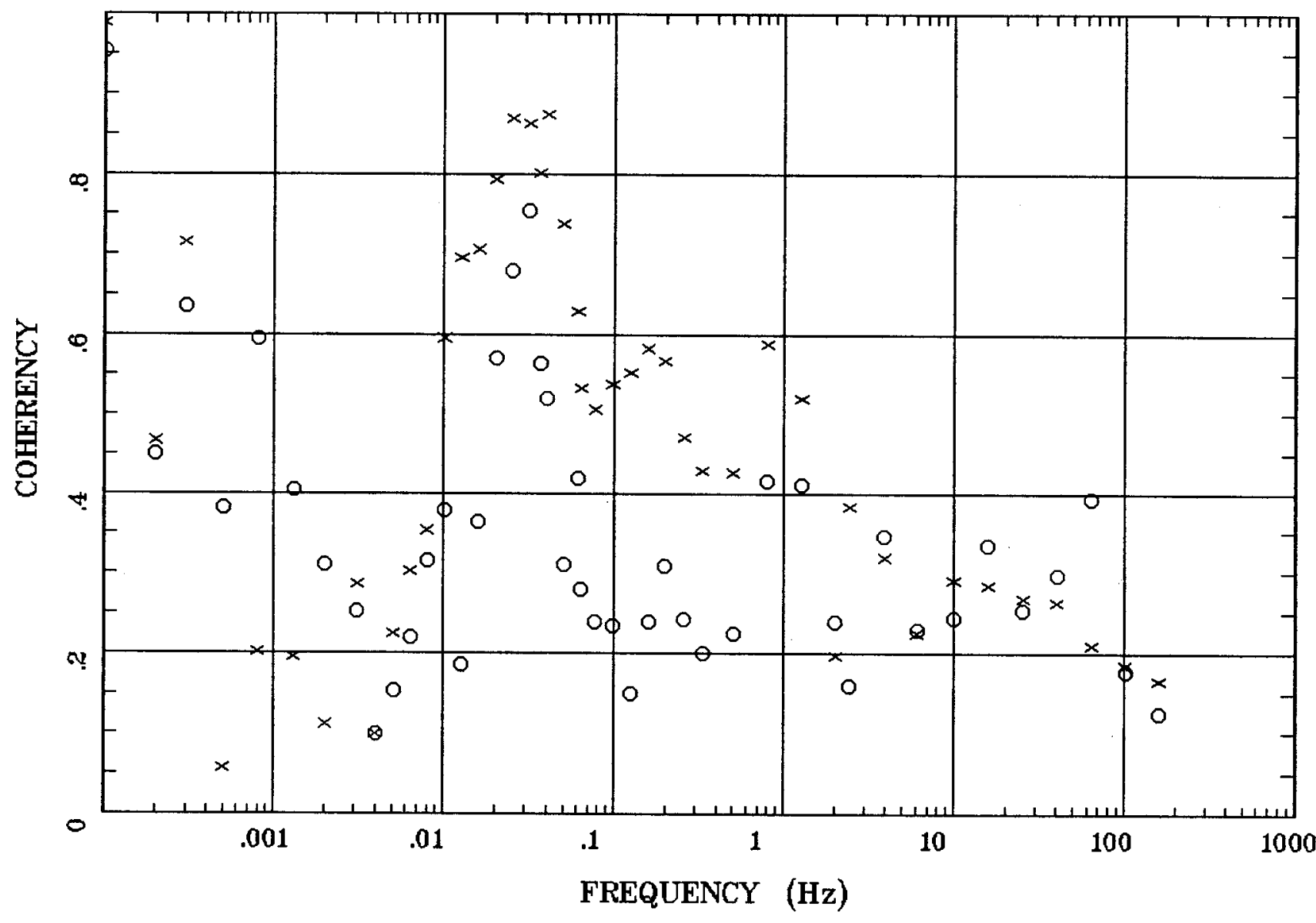

Client:

Remote:

Acquired: 19:5 Mar 08, 1998 Survey Co:
Rotation:

Filename: s4r16.a02

Channels: Ch1 Ch2 Ch3 Ch4 Ch5 Ch3 Ch4 Plotted: 10:59 Dec 07, 2004

< EMI - ElectroMagnetic Instruments 
APPARENT RESISTIVITY

Station 5

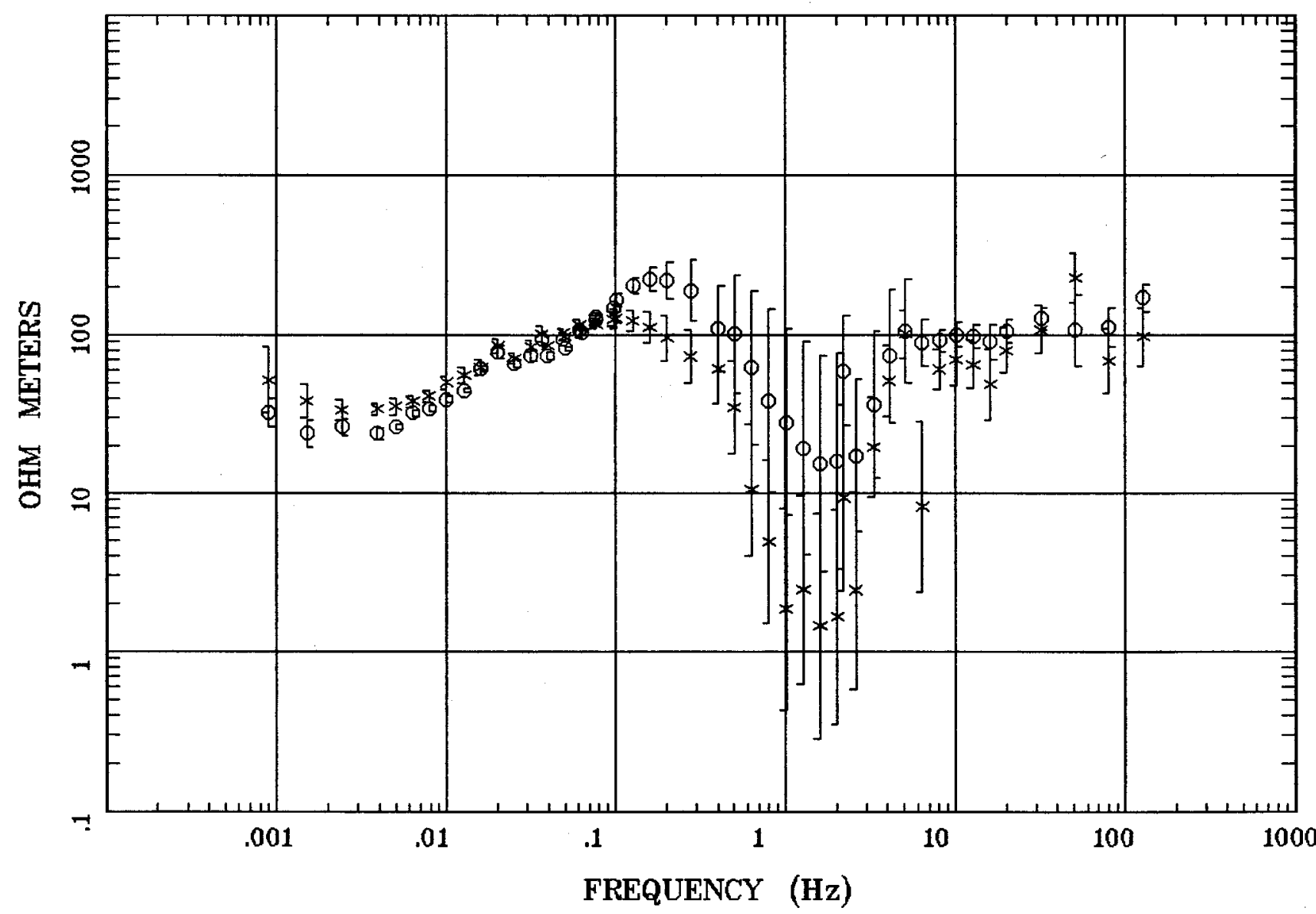

Client:

Remote:

Acquired: 19:5 Mar 08, 1998 Survey Co:
Rotation:

Filename: s5nr.a01

Channels: Ch1 Ch2 Ch3 Ch4 Ch5 Ch3 Ch4

Plotted: 11:02 Dec 07, 2004

$<$ EMI - ElectroMagnetic Instruments 


\section{IMPEDANCE PHASE}

Station 5

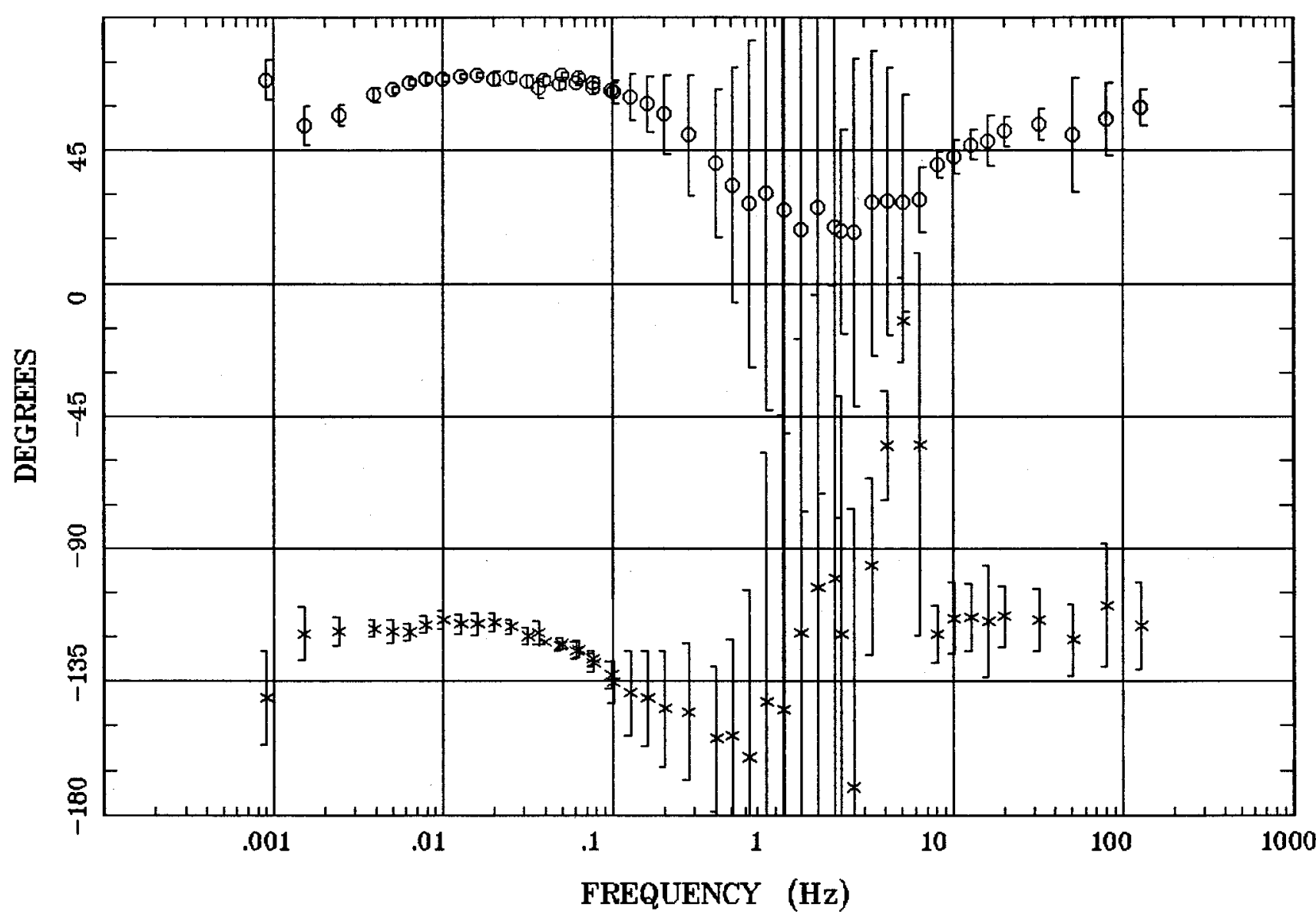

Client:

Remote:

Acquired: 19:5 Mar 08, 1998

Survey Co:
Rotation:

Filename: s5nr.a01

Channels: Ch1 Ch2 Ch3 Ch4 Ch5 Ch3 Ch4

Platted: 11:02 Dec 07, 2004

< EMI - ElectroMagnetic Instruments > 


\section{ROTATION ANGLE}

Station 5

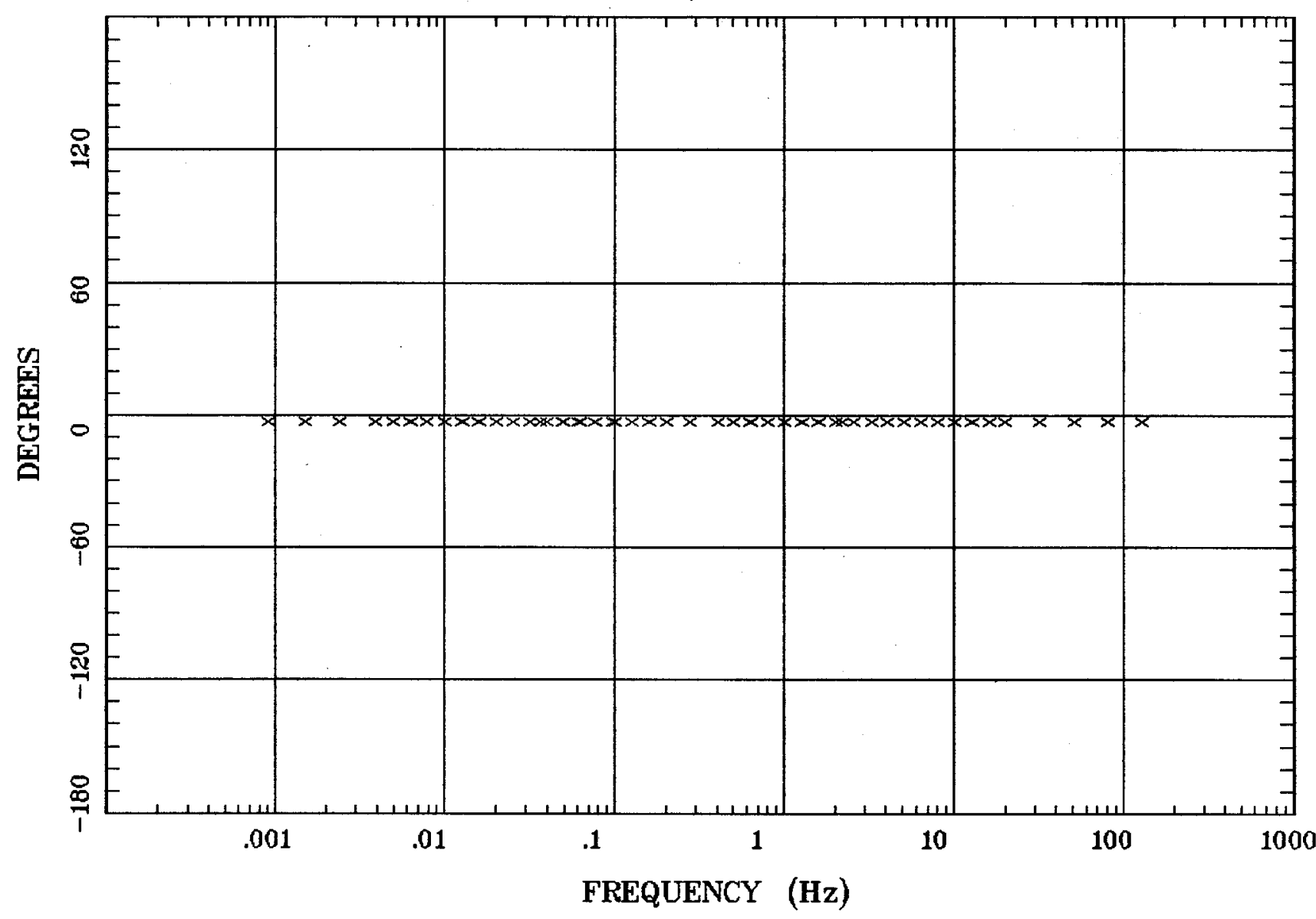

Client:

Remote:

Acquired: 19:5 Mar 08, 1998

Survey Ca:
Rotation:

Filename: s5nr.a01

Channels: Ch1 Ch2 Ch3 Ch4 Ch5 Ch3 Ch4

Plotted: 11:02 Dec 07, 2004

< EMI - ElectroMagnetic Instruments 


\section{IMPEDANCE SKEW}

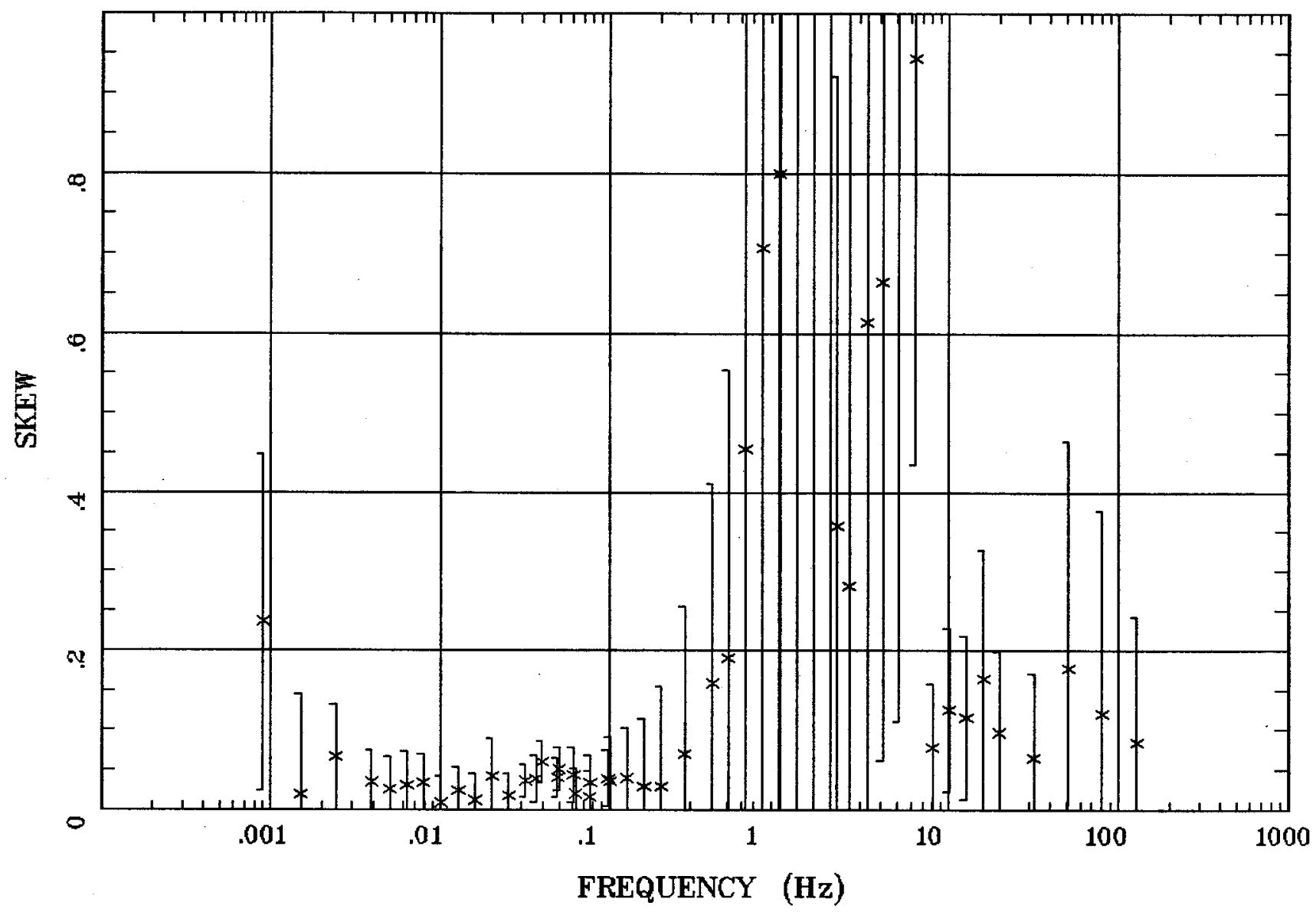

Client:

Remote:

Acquired: 19:5 Mar 08, 1998

Survey Co:
Rotation:

Filename: s5nr.a01

Channels: Ch1 Ch2 Ch3 Ch4 Ch5 Ch3 Ch4

Plotted: 11:02 Dec 0r, 2004

$<$ EMI - ElectroMagnetic Instruments > 
E MULT Coh.

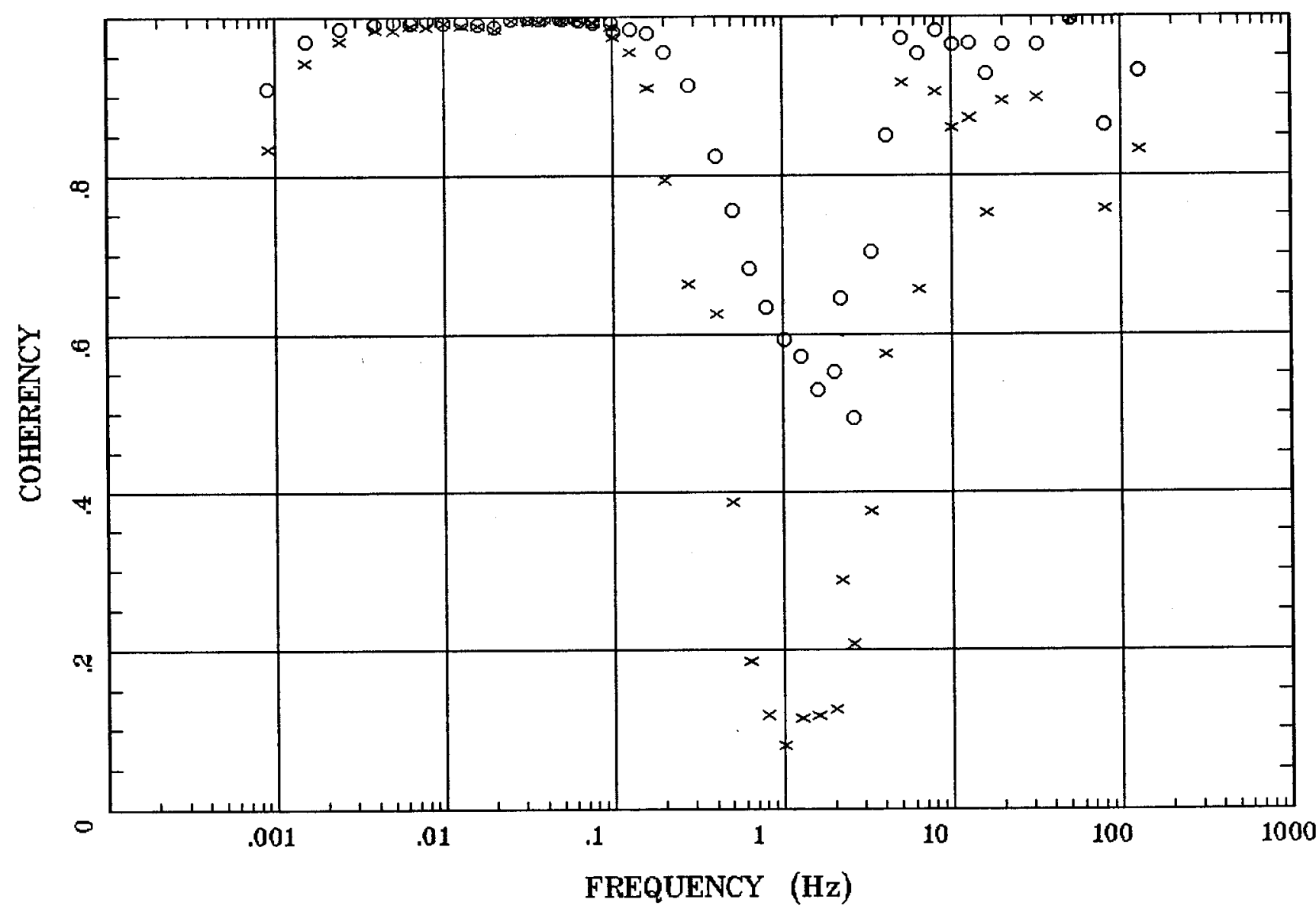

Client:

Remote:

Acquired: 19:5 Mar 08, 1998

Survey Co:
Ratation:

Filename: s5nr.a01

Channels: Ch1 Ch2 Ch3 Ch4 Ch5 Ch3 Ch4

Plotted: 11:02 Dec 07, 2004

$<$ EMI - ElectroMagnetic Instruments > 


\section{POLAR PLOTS}

Station 5

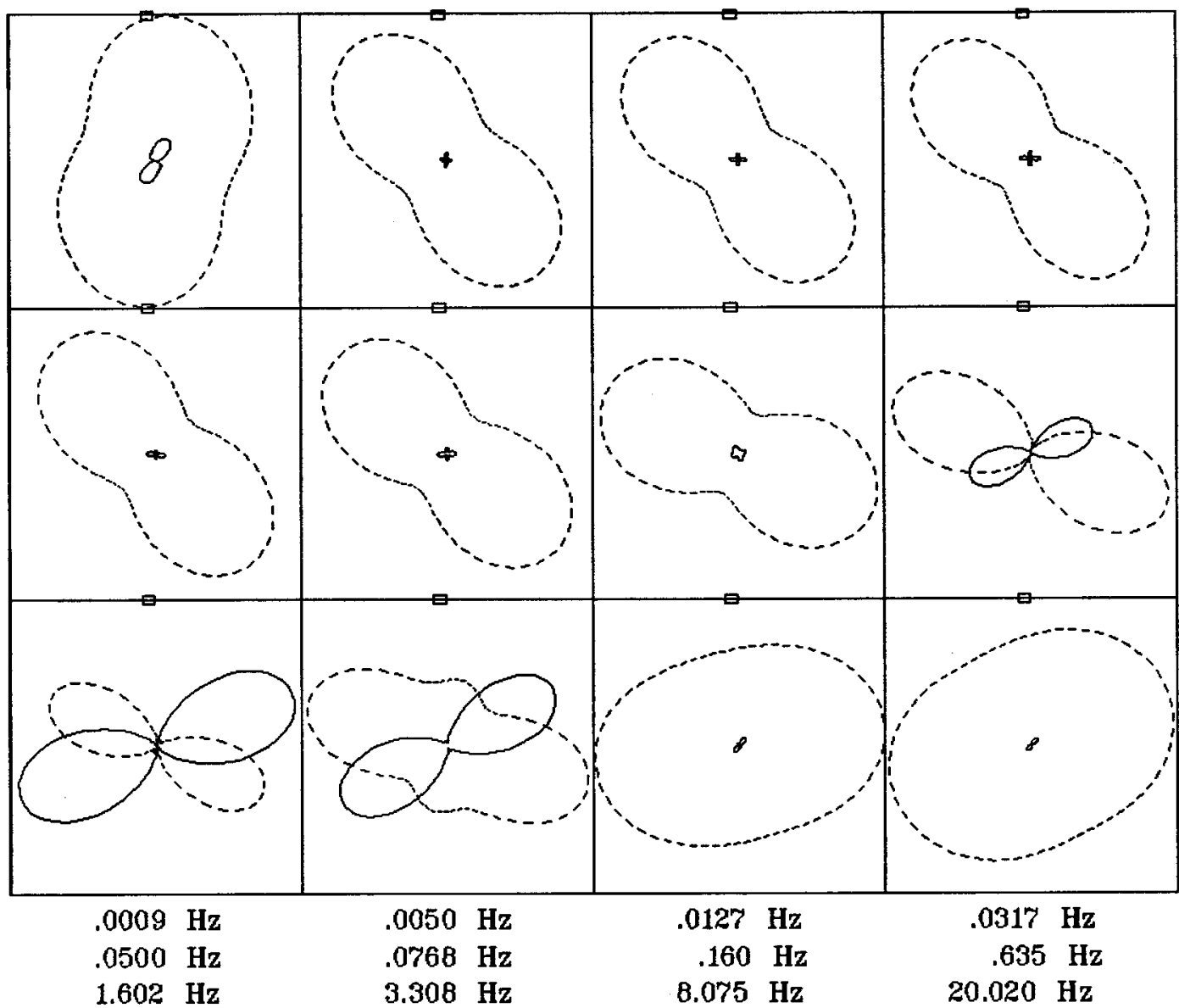

Client:

Remote:

Acquired: 19:5 Mar 08, 1998 Survey Co:
Rotation:

Filename: s5nr.a01

Channels: Ch1 Ch2 Ch3 Ch4 Ch5 Ch3 Ch4 Plotted: 11:02 Dec 07, 2004

< EMI - ElectroMagnetic Instruments > 
TIPPER MAGNITUDE

Station 5

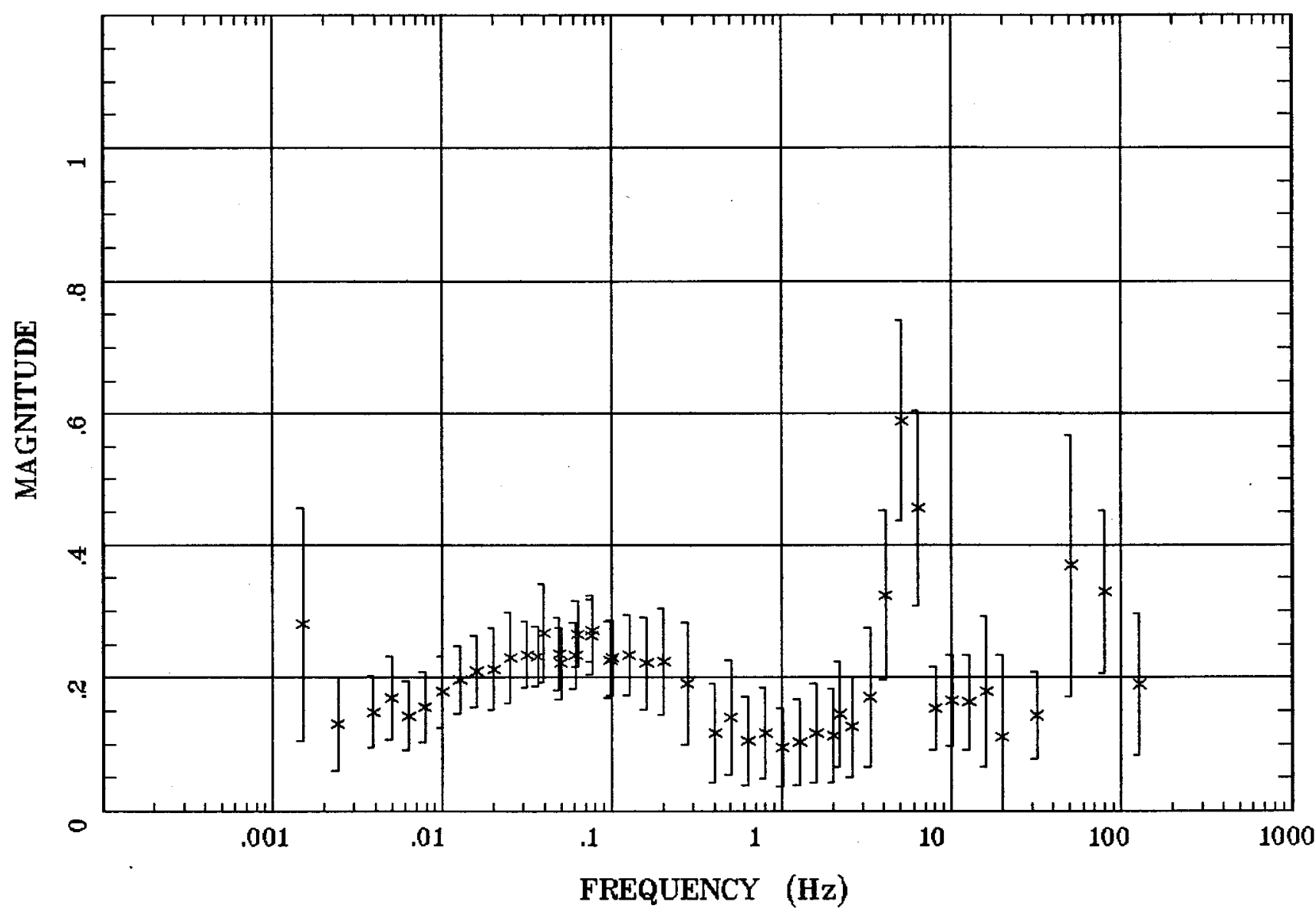

Client:

Remote:

Acquired: $19: 5$ Mar 08, 1998 Survey Co:

\section{Rotation:}

Filename: s5nr.a01

Channels: Ch1 Ch2 Ch3 Ch4 Ch5 Ch3 Ch4 Plotted: 11:02 Dec 07, 2004

< EMI - ElectroMagnetic Instruments 
TIPPER STRIKE

\section{Station 5}

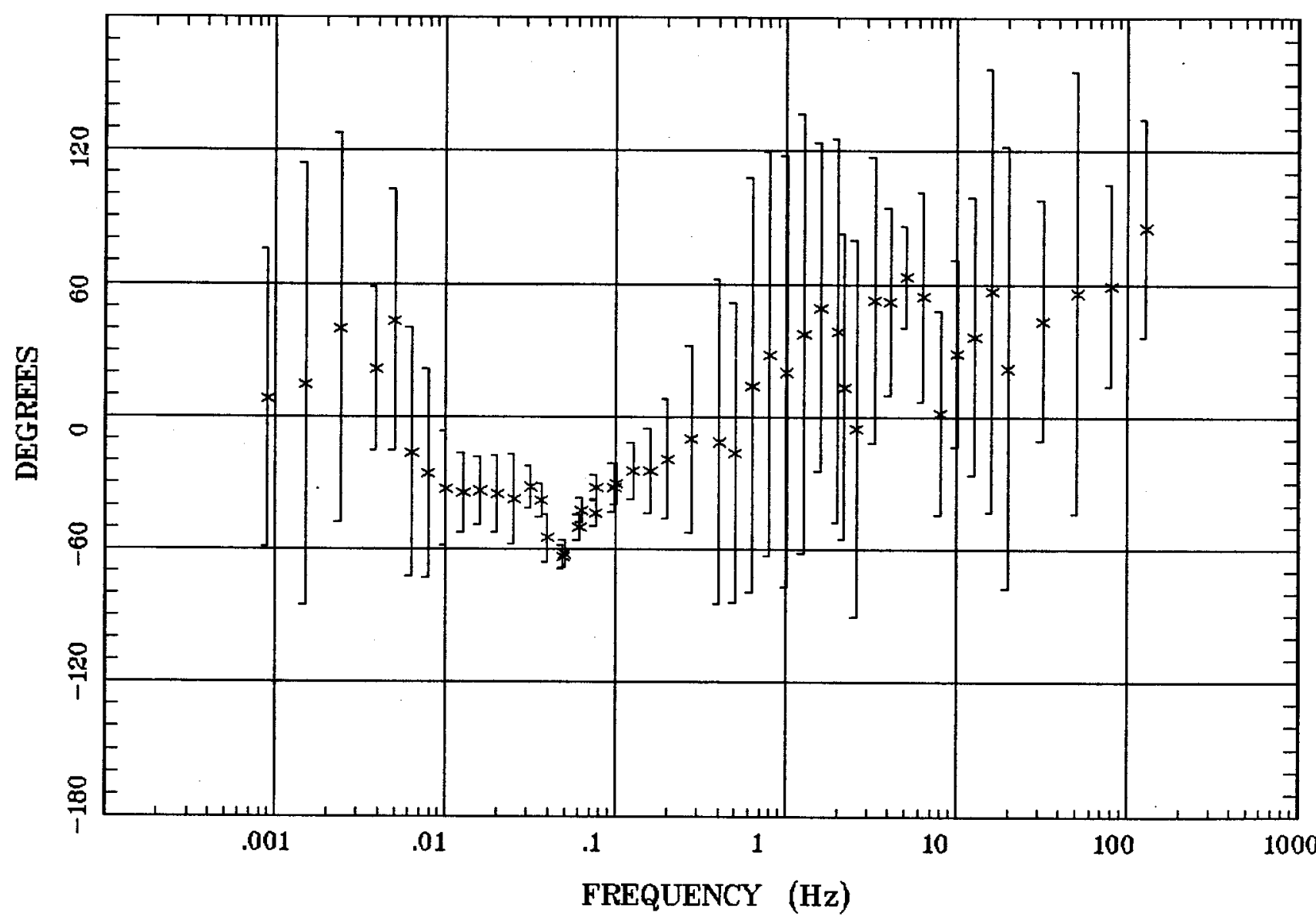

Client:

Remote:

Acquired: 19:5 Mar 08, 1998

Survey Co:
Rotation:

Filename: s5nr.a01

Channels: Ch1 Ch2 Ch3 Ch4 Ch5 Ch3 Ch4

Plotted: 11:02 Dec 07, 2004

< EMI - ElectroMagnetic Instruments 
HzHx.x Coh HzHy.o

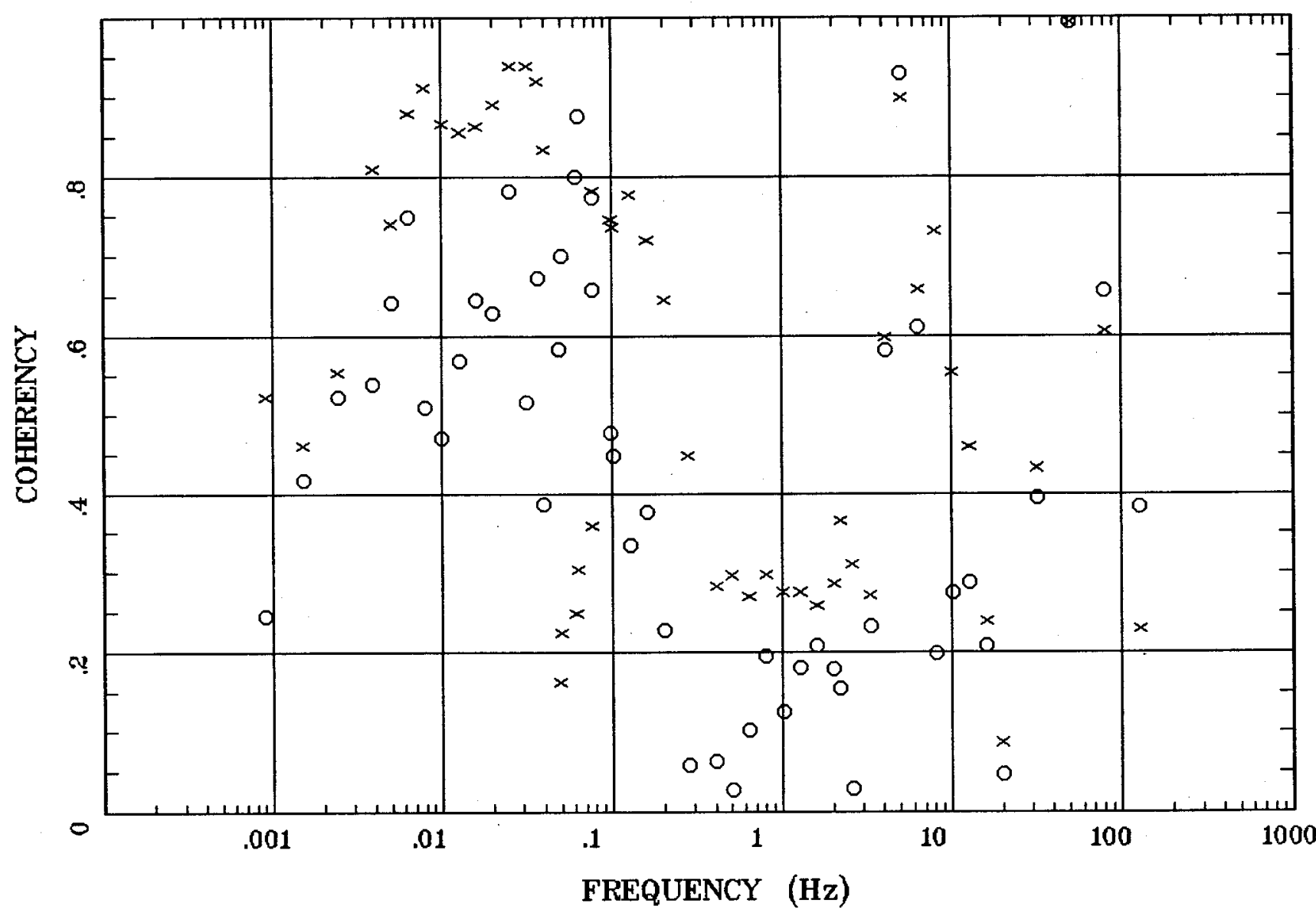

Client:

Remote:

Acquired: 19:5 Mar 08, 1998

Survey Co:
Rotation:

Filename: s5nr.a01

Channels: Ch1 Ch2 Ch3 Ch4 Ch5 Ch3 Ch4

Plotted: 11:02 Dec 07, 2004

< EMI - ElectroMagnetic Instruments 


\section{APPARENT RESISTIVITY}

Station 6

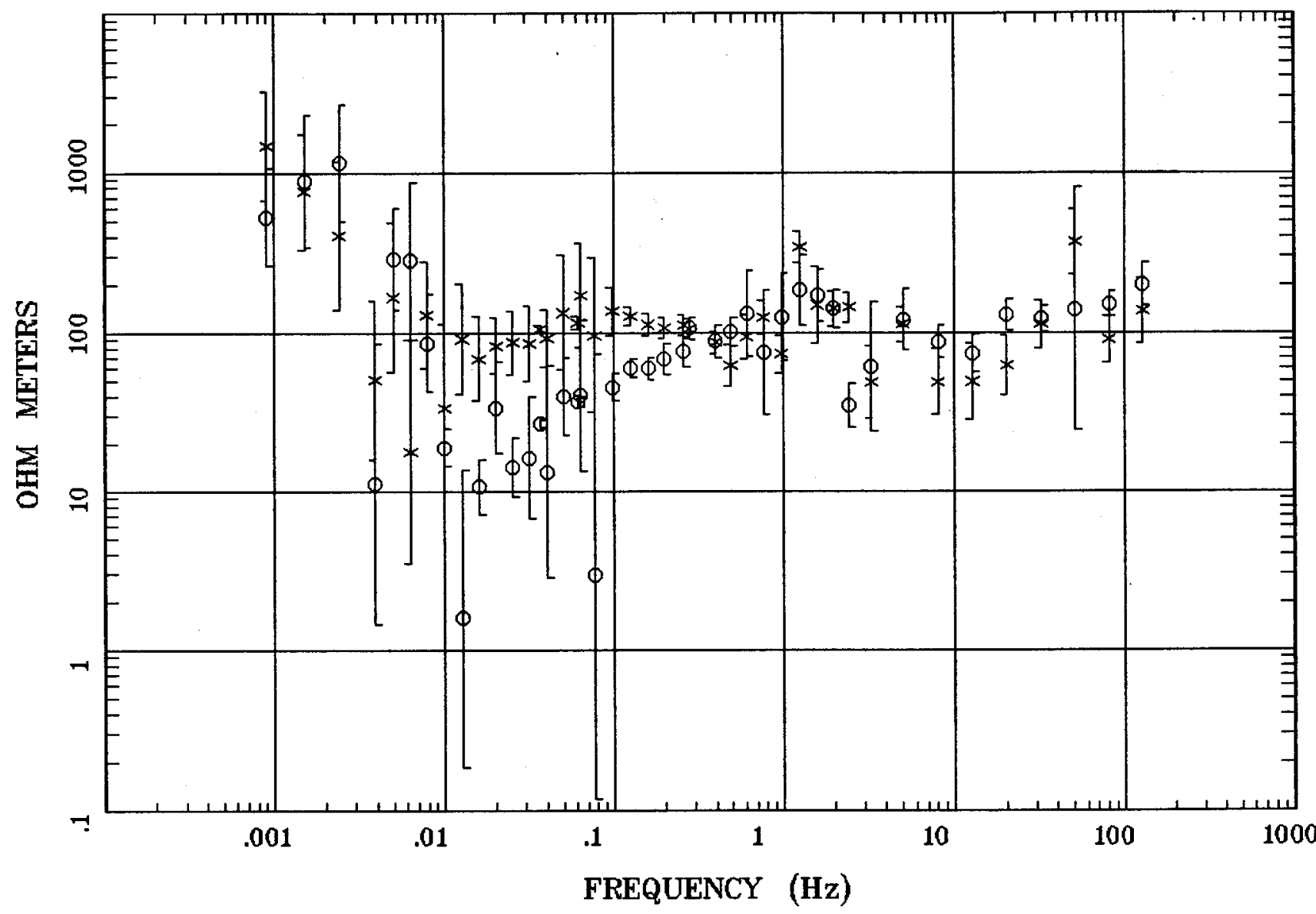

Client:

Remote:

Acquired: 19:5 Mar 08, 1998 Survey Co:
Rotation:

Filename: s6r36.a01

Channels: Ch1 Ch2 Ch3 Ch4 Ch5 Ch3 Ch4 Plotted: 10:59 Dec 07, 2004

$<$ EMI - ElectroMagnetic Instruments 


\section{IMPEDANCE PHASE}

\section{Station 6}

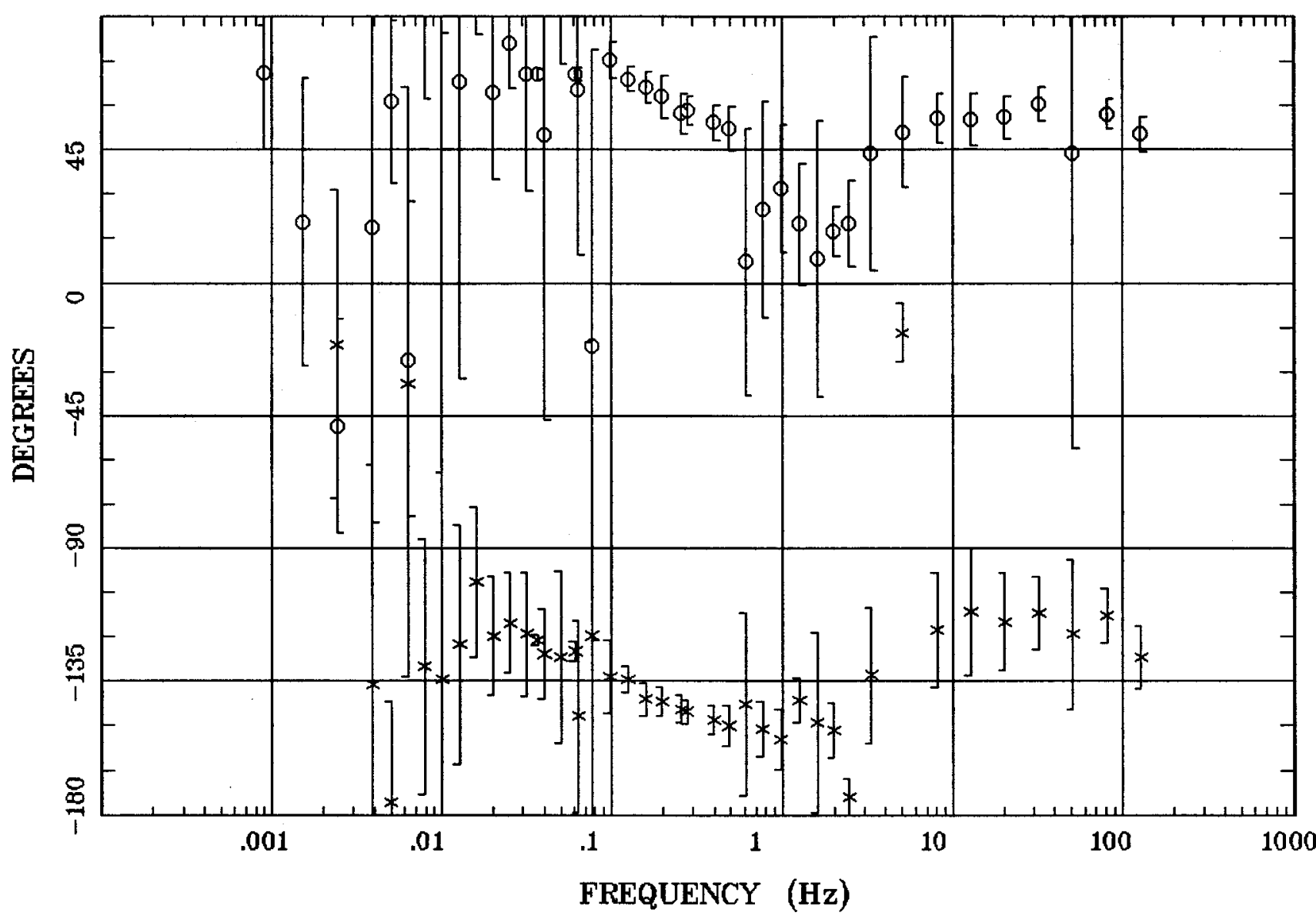

Client:

Remote:

Acquired: 19:5 Mar 08, 1998 Survey Co:
Rotation:

Filename: s6r36.a01

Channels: Ch1 Ch2 Ch3 Ch4 Ch5 Ch3 Ch4 Platted: 10:59 Dec 07, 2004

$<$ EMI - ElectroMagnetic Instruments 


\section{ROTATION ANGLE}

Station 6

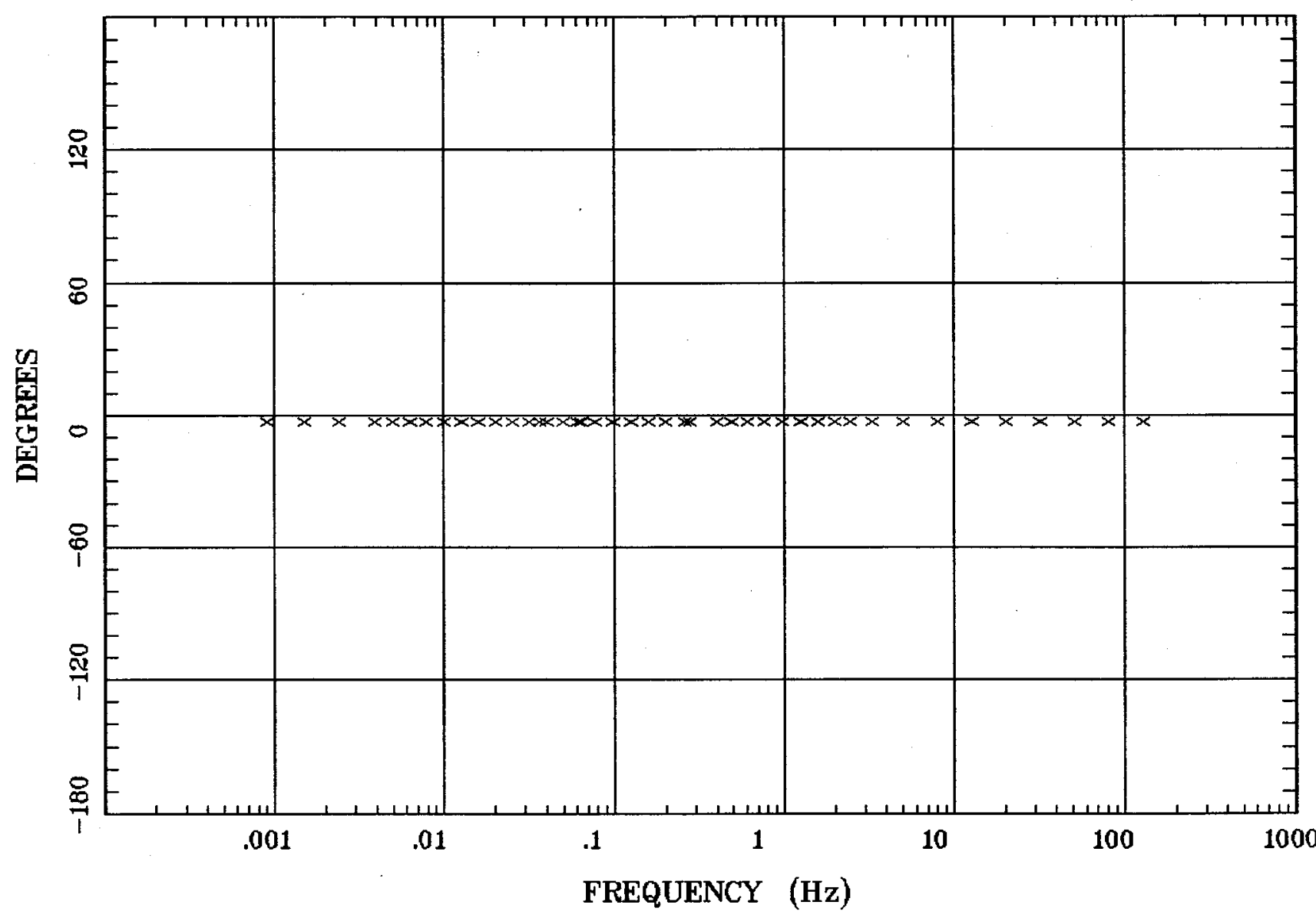

Client:

Remote:

Acquired: 19:5 Mar 08, 1998

Survey Co:
Rotation:

Filename: s6r36.a01

Channels: Ch1 Ch2 Ch3 Ch4 Ch5 Ch3 Ch4

Plotted: 10:59 Dec 07, 2004

<EMI - ElectroMagnetic Instruments > 


\section{IMPEDANCE SKEW}

Station 6

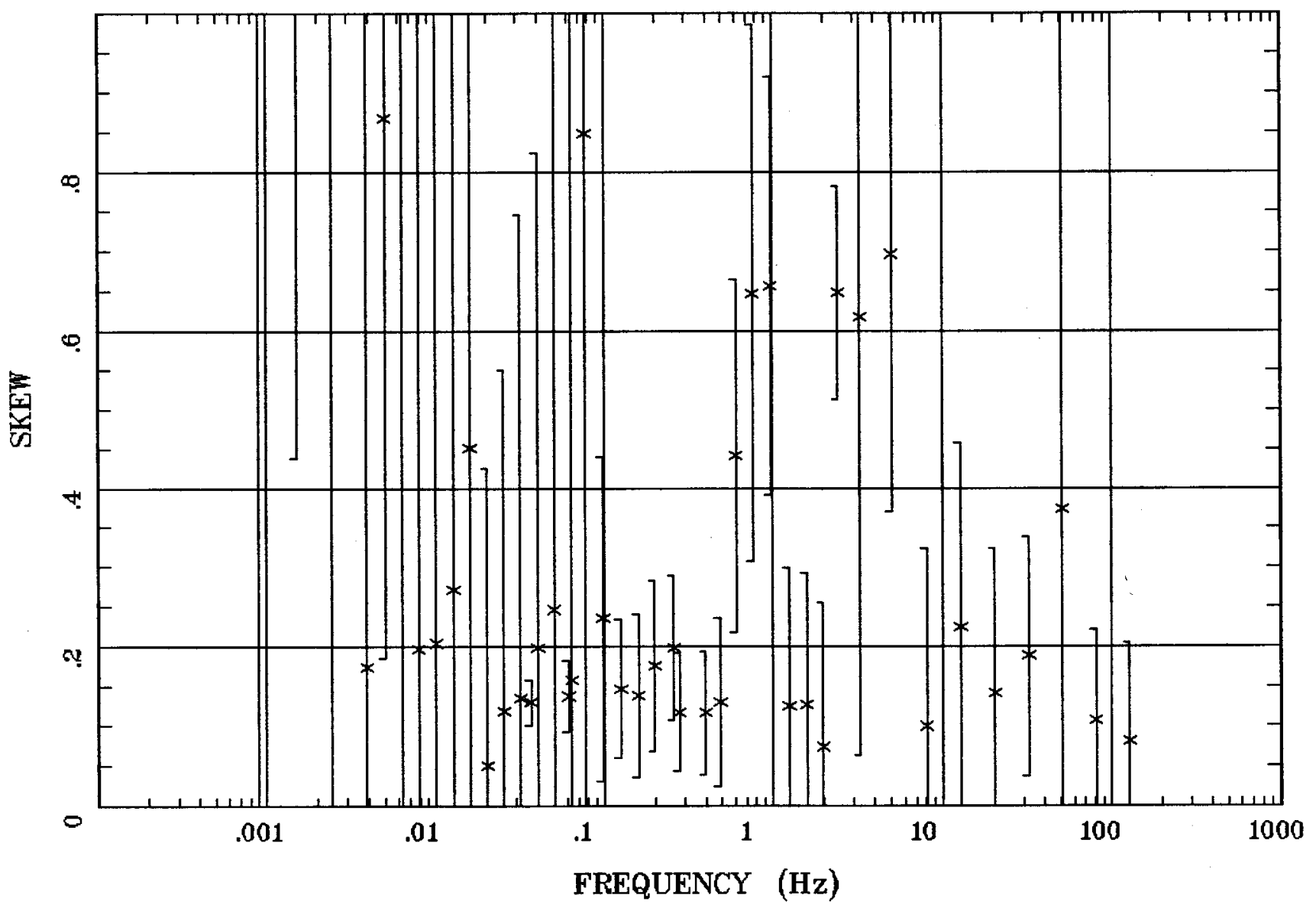

Client:

Rotation:

Remote:

Filename: s6r36.a01

Acquired: 19:5 Mar 08, 1998

Channels: Ch1 Ch2 Ch3 Ch4 Ch5 Ch3 Ch4

Platted: 10:59 Dec 07, 2004

Survey Co:

< EMI - ElectroMagnetic Instruments > 
E MULT Coh.

Station 6

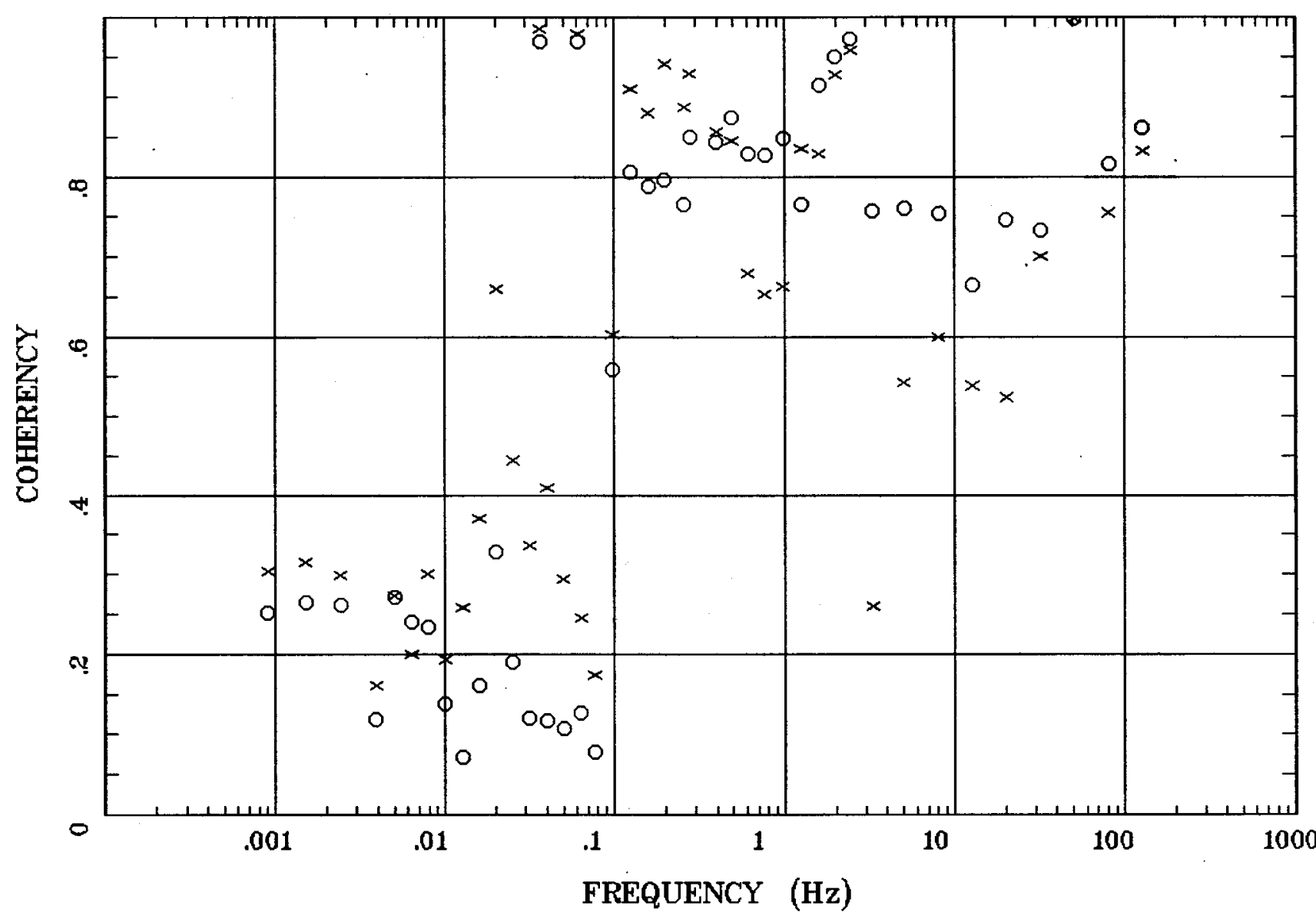

Client: Remote:

Acquired: 19:5 Mar 08, 1998 Survey Co:
Rotation:

Filename: $56 \mathrm{r} 36 . \mathrm{a} 01$

Channels: Ch1 Ch2 Ch3 Ch4 Ch5 Ch3 Ch4 Plotted: 10:59 Dec 07, 2004

< EMI - ElectroMagnetic Instruments 
POLAR PLOTS

Station 6

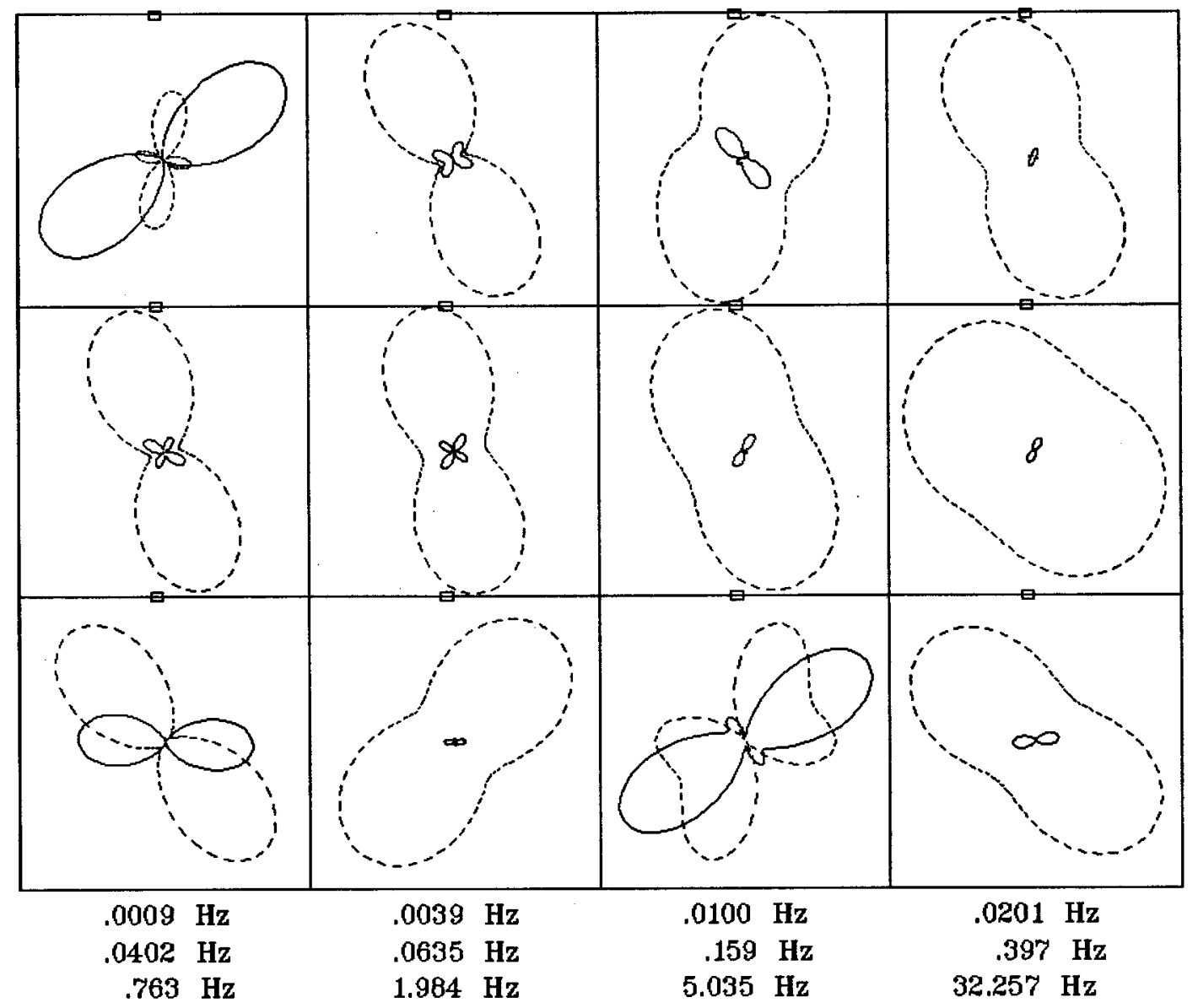

Client:

Remote:

Acquired: 19:5 Mar 08, 1998 Survey Co:
Rotation:

Filename: s6r36.a01

Channels: Ch1 Ch2 Ch3 Ch4 Ch5 Ch3 Ch4

Plotted: 10:59 Dec 07, 2004

< EMI - ElectroMagnetic Instruments 


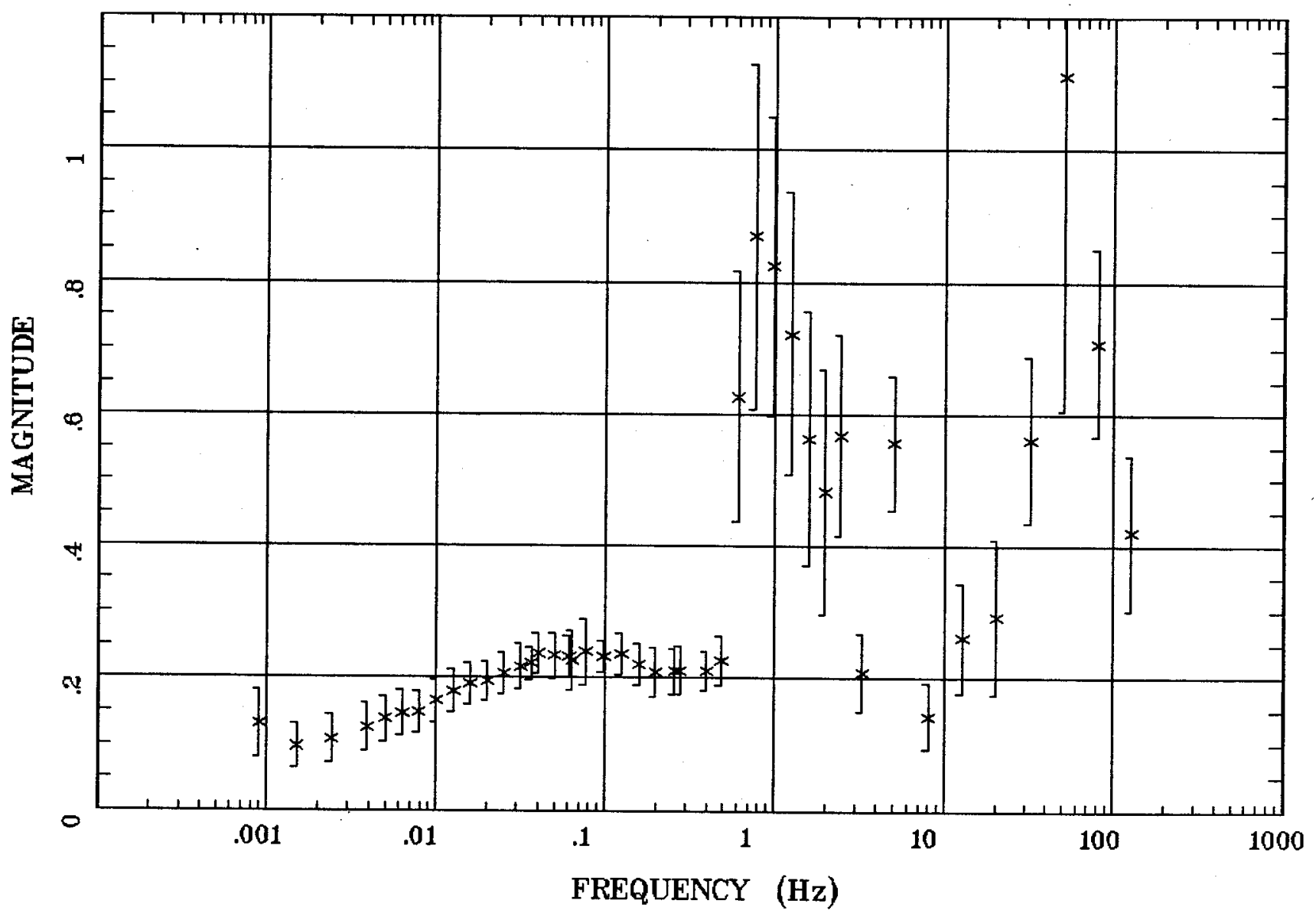

Client:

Remote:

Acquired: 19:5 Mar 08, 1998

Survey $\mathrm{Co}$
Rotation:

Filename: s6r36.a01

Channels: Ch1 Ch2 Ch3 Ch4 Ch5 Ch3 Ch4

Plotted: 10:59 Dec 07, 2004

< EMI - ElectroMagnetic Instruments 
TIPPER STRIKE

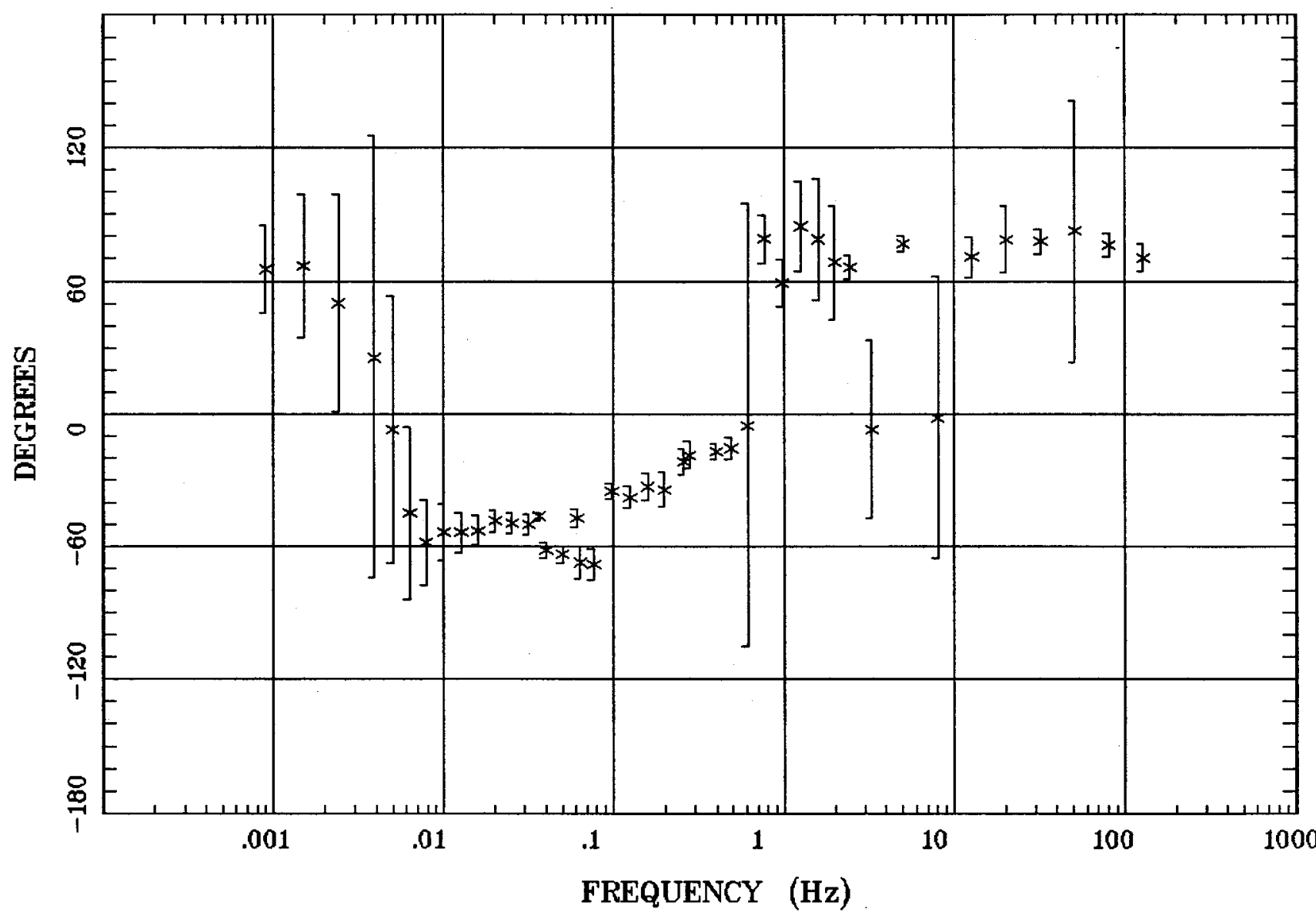

Client:

Remote:

Acquired: 19:5 Mar 08, 1998 Survey Co:
Rotation:

Filename: s6r36.a01

Channels: Ch1 Ch2 Ch3 Ch4 Ch5 Ch3 Ch4 Plotted: 11:00 Dec 07, 2004

$<$ EMI - ElectroMagnetic Instruments 


\section{Station 6}

\section{HzHx.x Coh HzHy.o}

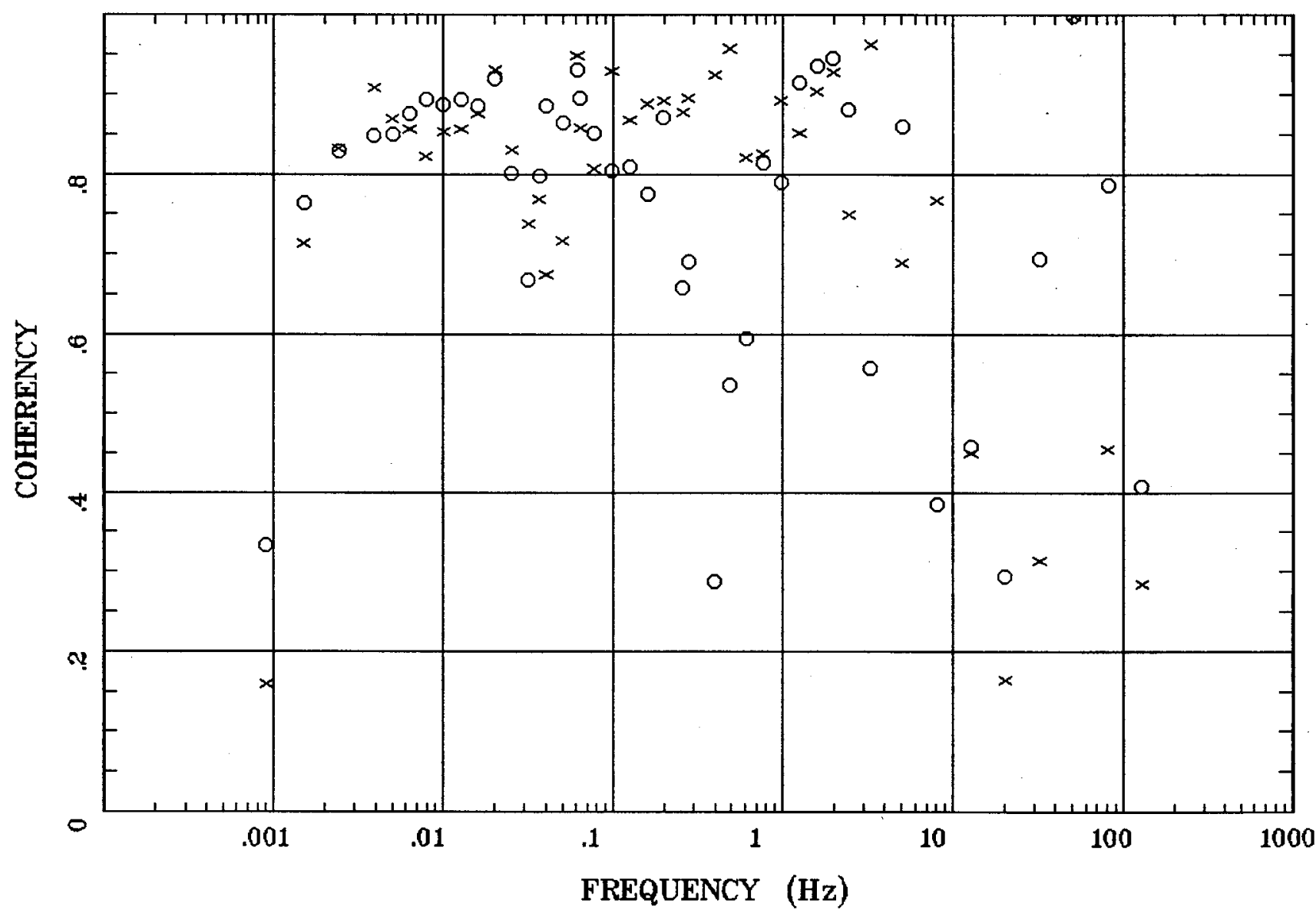

Client:

Remote:

Acquired: 19:5 Mar 08, 1998 Survey Co:
Rotation:

Filename: s6r36.a01

Channels: Ch1 Ch2 Ch3 Ch4 Ch5 Ch3 Ch4 Plotted: 11:00 Dec 07, 2004

< EMI - ElectroMagnetic Instruments 


\section{APPARENT RESISTIVITY}

Station 7

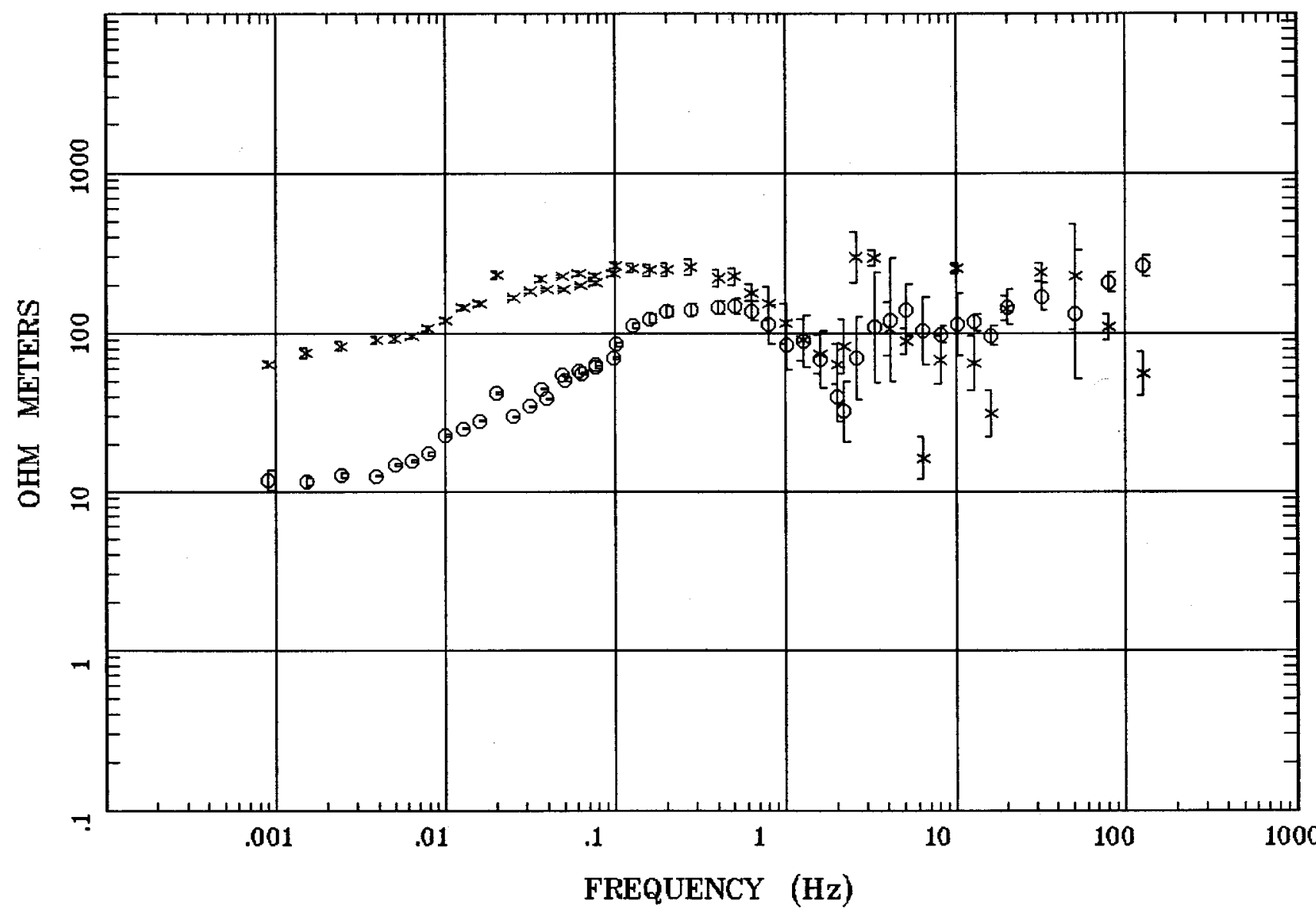

Client:

Remote:

Acquired: 19:5 Mar 08, 1998 Survey Co:
Rotation:

Filename: s7r30.a01

Channels: Ch1 Ch2 Ch3 Ch4 Ch5 Ch3 Ch4 Plotted: 11:00 Dec 07, 2004

< EMI - ElectroMagnetic Instruments 


\section{IMPEDANCE PHASE}

Station 7

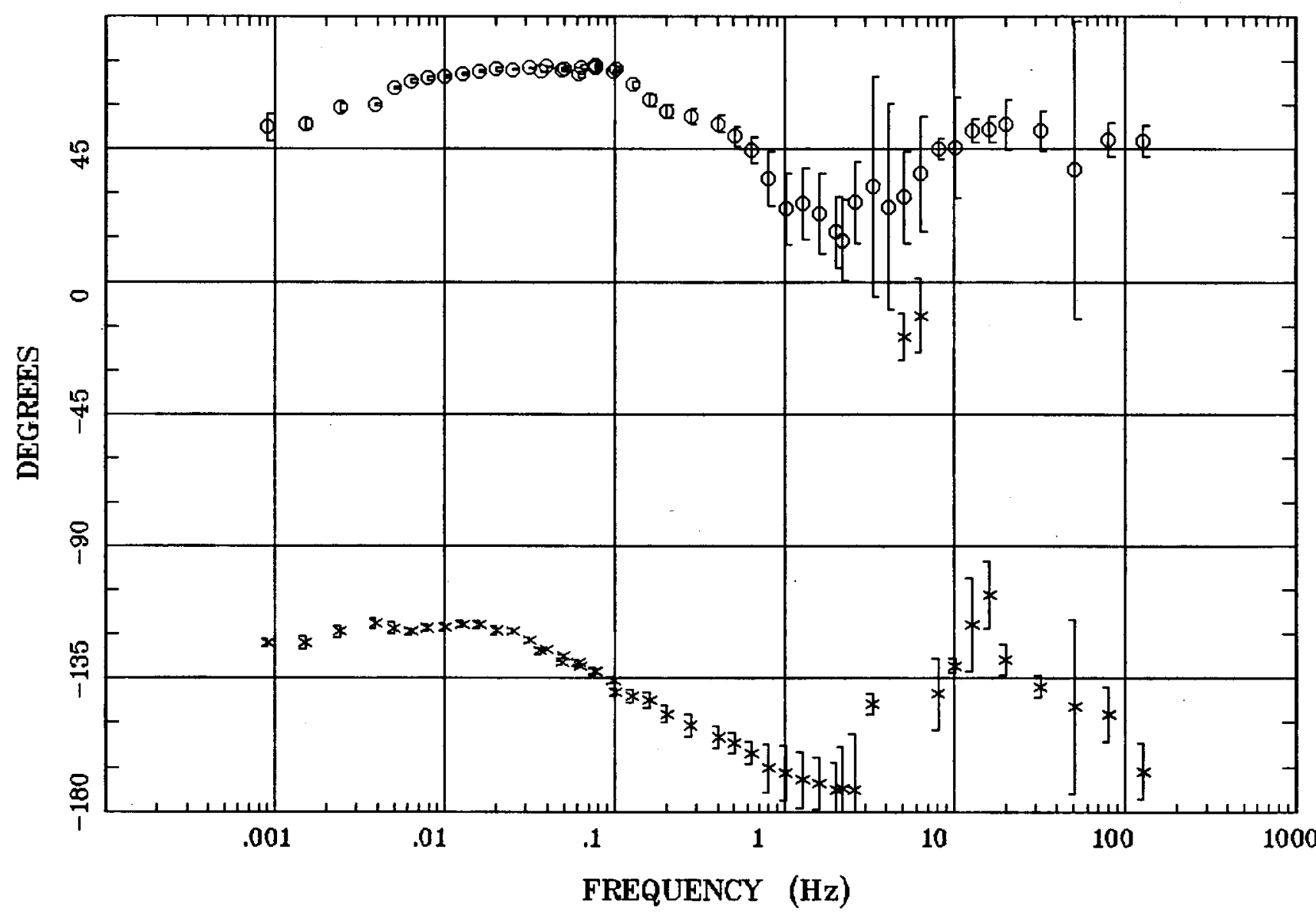

Client:

Remote:

Acquired: 19:5 Mar 08, 1998 Survey Co:
Rotation:

Filename: s7r30.a01

Channels: Ch1 Ch2 Ch3 Ch4 Ch5 Ch3 Ch4 Plotted: 11:00 Dec 07, 2004

< EMI - ElectroMagnetic Instruments 


\section{ROTATION ANGLE}

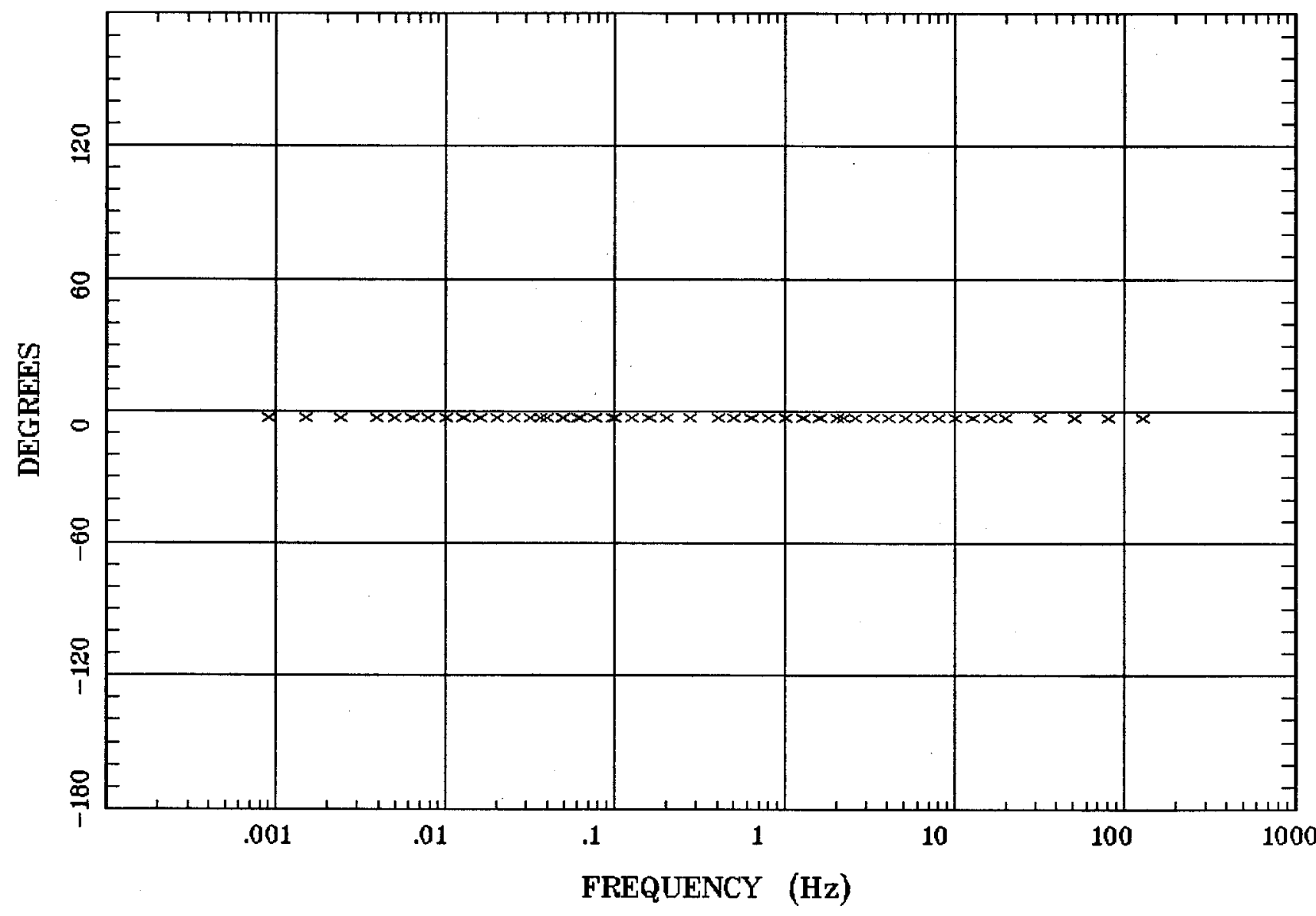

Client:

Remote:

Acquired: 19:5 Mar 08, 1998 Survey Co:
Rotation:

Filename: s7r30.a01

Channels: Ch1 Ch2 Ch3 Ch4 Ch5 Ch3 Ch4 Plotted: 11:00 Dec 07, 2004

< EMI - ElectroMagnetic Instruments 
Station 7

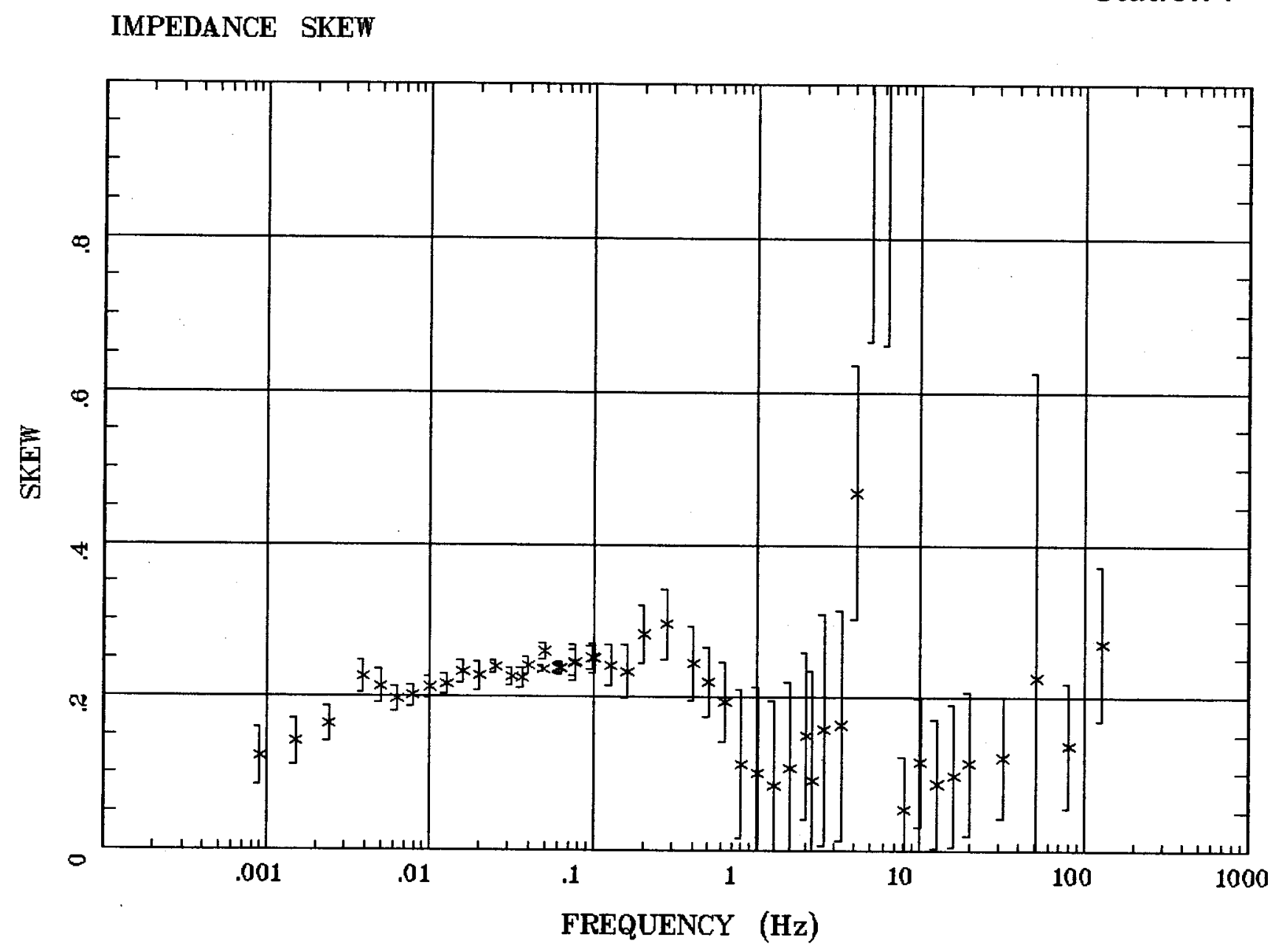

Client:

Remote:

Acquired: 19:5 Mar 08, 1998

Survey Co:
Rotation:

Filename: s7r30.a01

Channels: Ch1 Ch2 Ch3 Ch4 Ch5 Ch3 Ch4

Plotted: 11:00 Dec 07, 2004

$<$ EMI - ElectroMagnetic Instruments 


\section{E MULT Coh.}

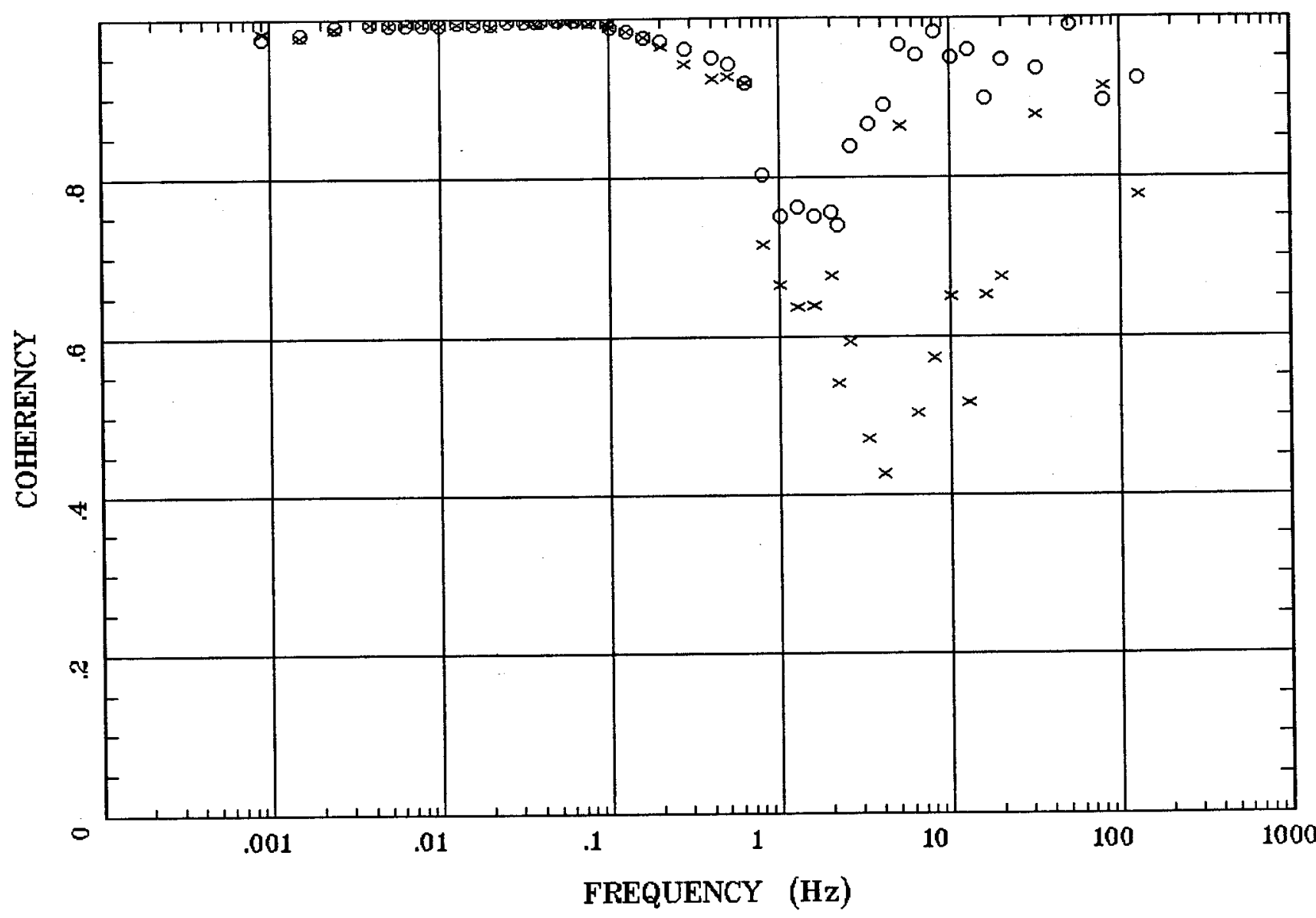

Client:

Remote:

Acquired: 19:5 Mar 08, 1998

Survey Co:

\section{Rotation:}

Filename: $s 7 \mathrm{r} 30 . \mathrm{a} 01$

Channels: Ch1 Ch2 Ch3 Ch4 Ch5 Ch3 Ch4 Plotted: 11:00 Dec 07, 2004

< EMI - ElectroMagnetic Instruments 


\section{POLAR PLOTS}

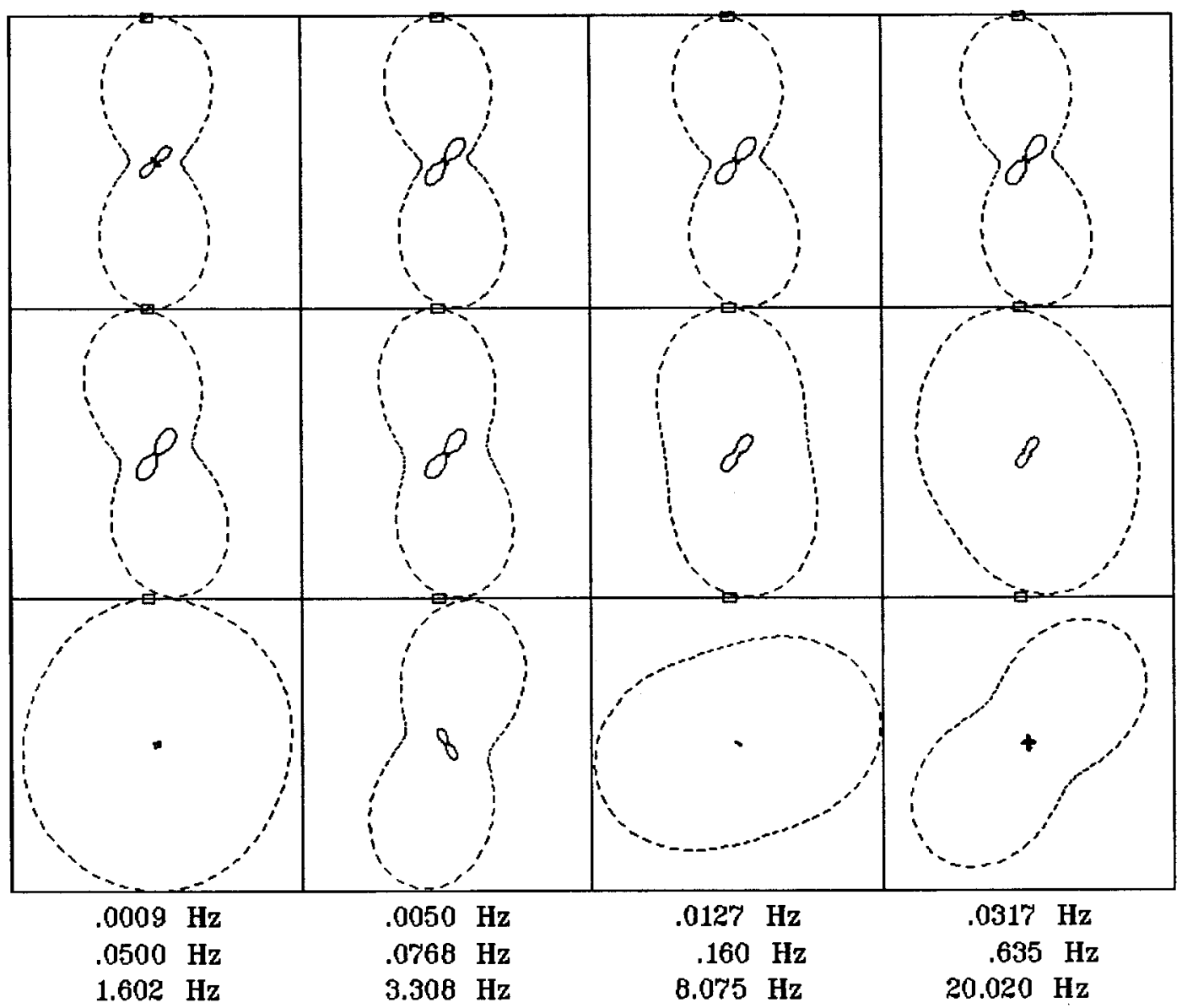

Client:

Remote:

Acquired: 19:5 Mar 08, 1998 Survey Co:
Rotation:

Filename: s7r30.a01

Channels: Ch1 Ch2 Ch3 Ch4 Ch5 Ch3 Ch4 Platted: 11:00 Dec 07, 2004

$<$ EMI - ElectroMagnetic Instruments > 


\section{TIPPER MAGNITUDE}

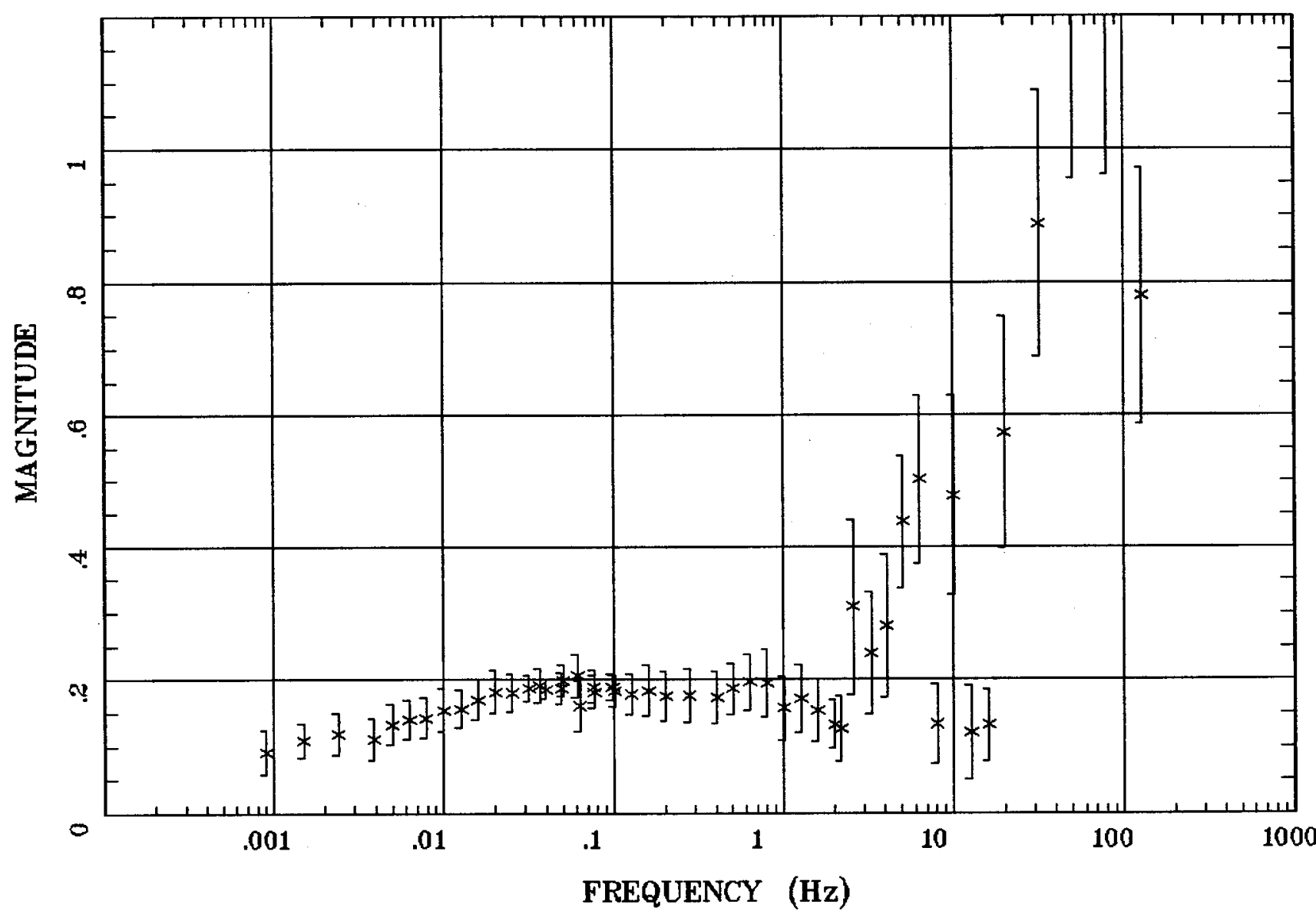

Client:

Remote:

Acquired: 19:5 Mar 08, 1998 Survey Co:
Rotation:

Filename: s7r30.a01

Channels: Ch1 Ch2 Ch3 Ch4 Ch5 Ch3 Ch4 Plotted: 11:00 Dec 07, 2004

< EMI - ElectroMagnetic Instruments 


\section{TIPPER STRIKE}

Station 7

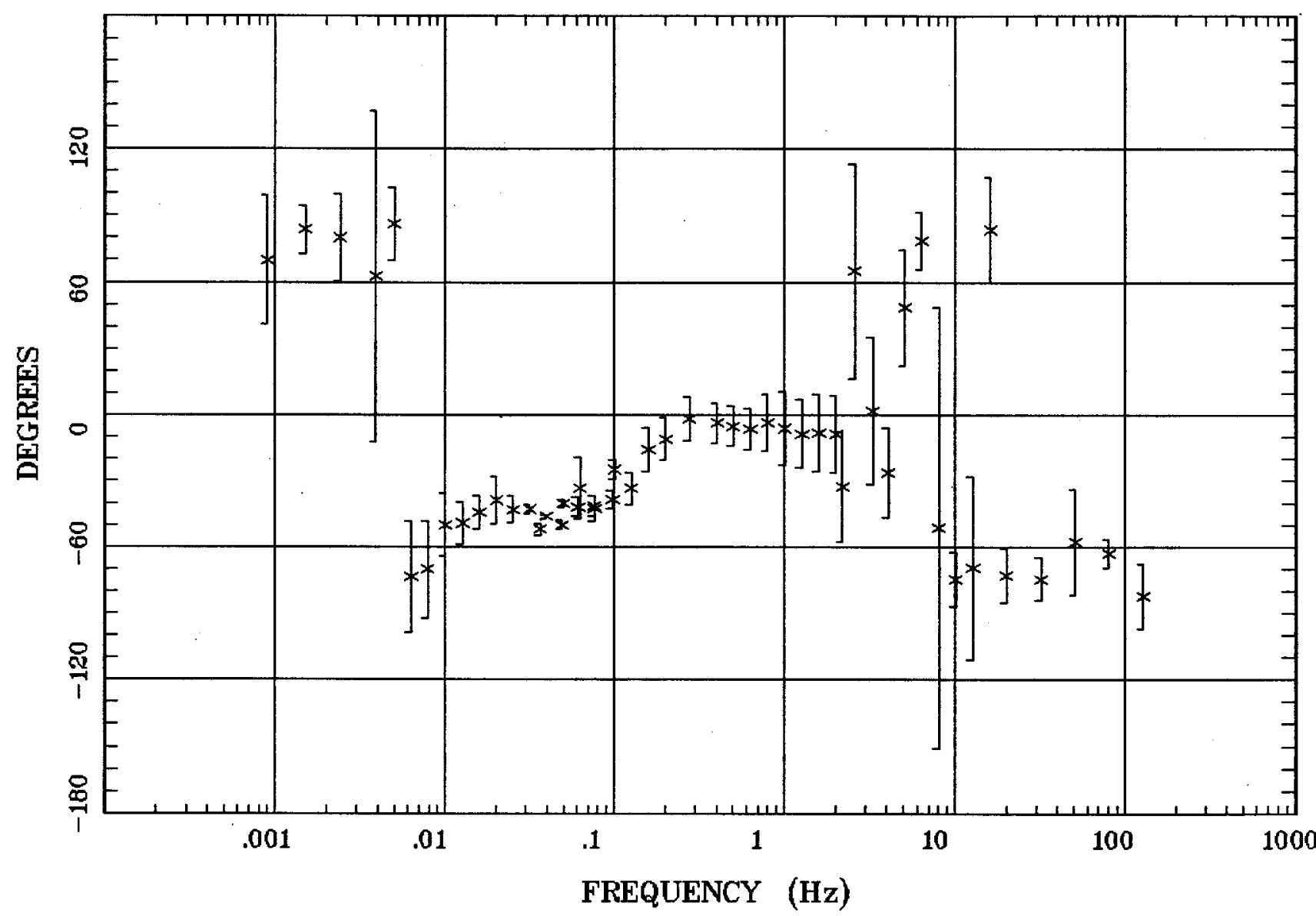

Client:

Remote:

Acquired: 19:5 Mar 08, 1998 Survey Co:
Rotation:

Filename: s7r30.a01

Channels: Ch1 Ch2 Ch3 Ch4 Ch5 Ch3 Ch4

Plotted: 11:00 Dec 07, 2004

\& EMI - ElectroMagnetic Instruments 


\section{HzHx.x Coh HzHy.o}

\section{Station 7}

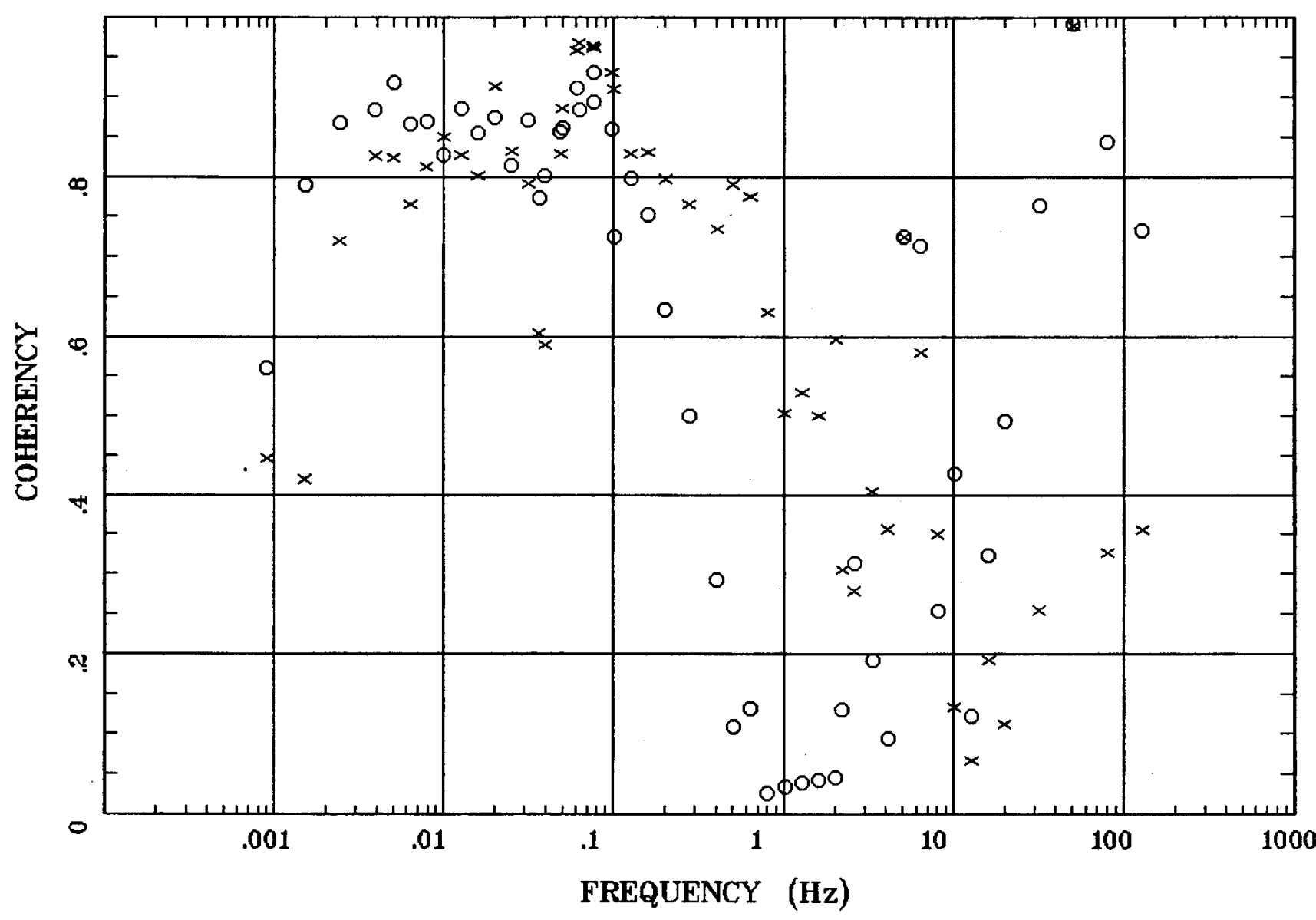

Client:

Remote:

Acquired: 19:5 Mar 08, 1998 Survey Co:
Rotation:

Filename: s7r30.a01

Channels: Ch1 Ch2 Ch3 Ch4 Ch5 Ch3 Ch4

Plotted: 11:00 Dec 07, 2004

< EMI - ElectroMagnetic Instruments 


\section{APPARENT RESISTIVITY}

Station 8

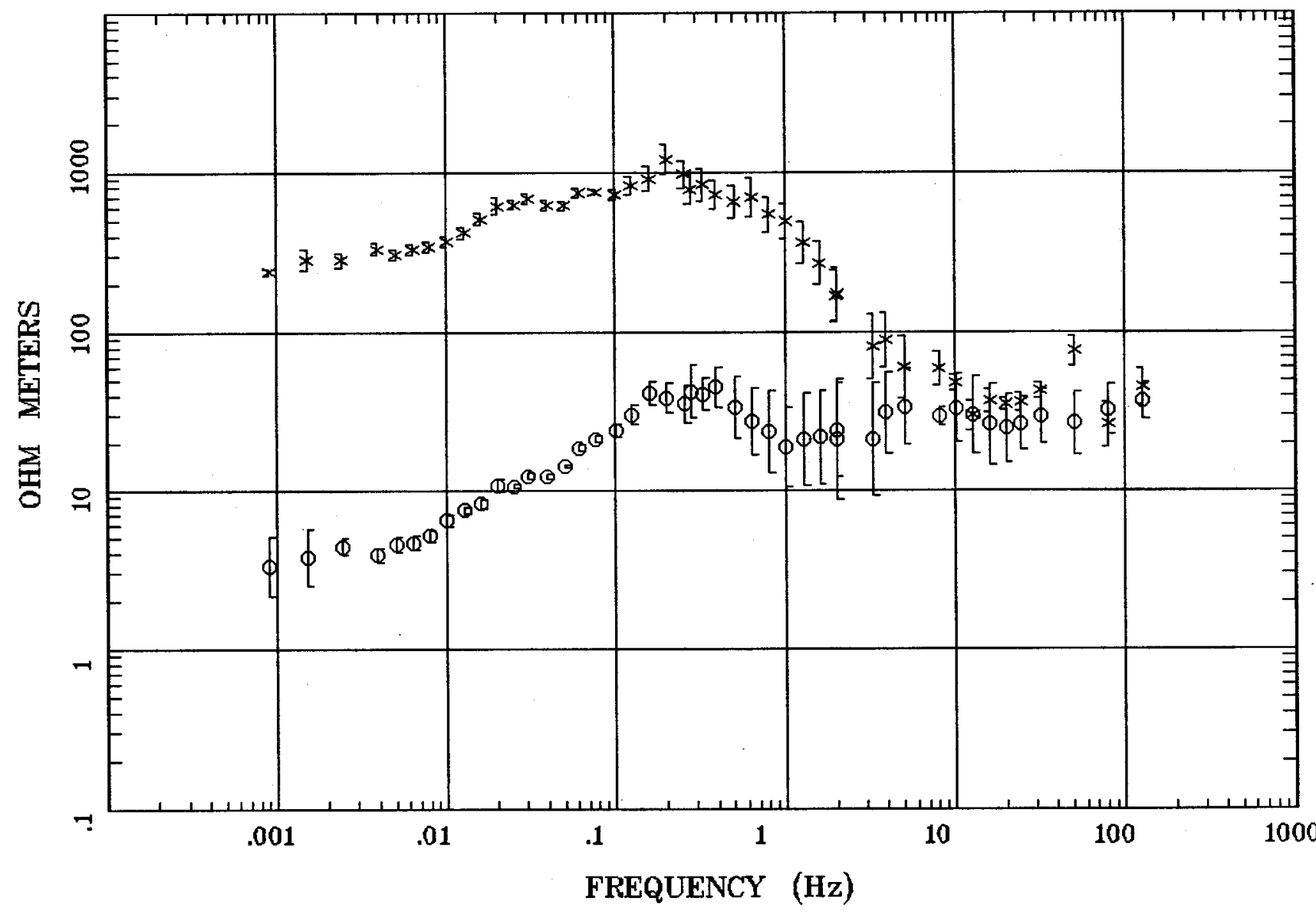

Client:

Remote:

Acquired: 19:5 Mar 08, 1998 Survey Co:
Rotation:

Filename: s8ed.avg

Channels: Ch1 Ch2 Ch3 Ch4 Ch5 Ch3 Ch4 Plotted: 10:18 Dec 13, 2004

$<$ EMI - ElectroMagnetic Instruments > 


\section{IMPEDANCE PHASE}

Station 8

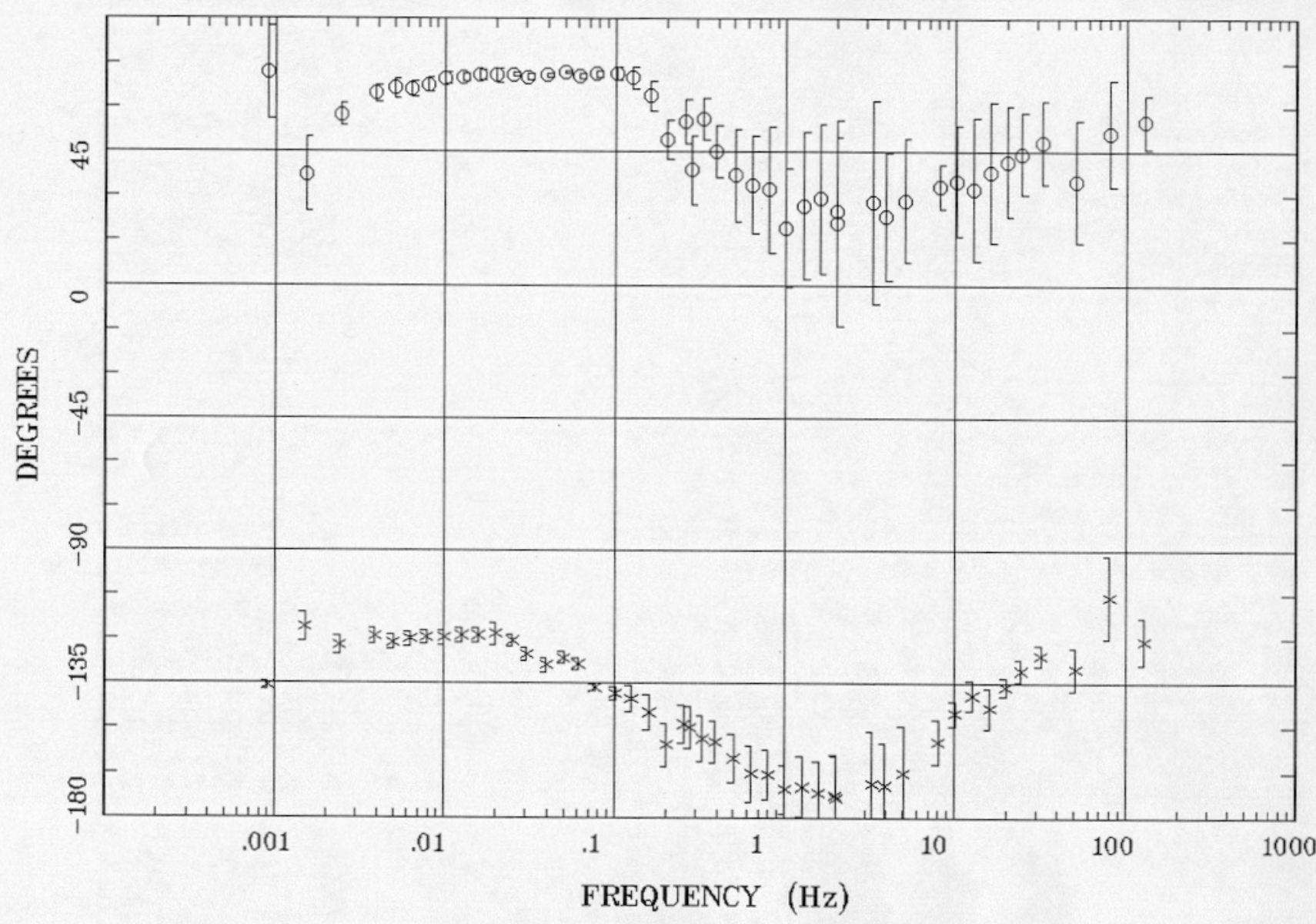

Client:

Remote:

Acquired: 19:5 Mar 08, 1998

Survey Co:
Rotation:

Filename: sBed.avg

Channels: Ch1 Ch2 Ch3 Ch4 Ch5 Ch3 Ch4

Plotted: 10:18 Dec 13, 2004

$<$ EMI - ElectroMagnetic Instruments 


\section{ROTATION ANGLE}

Station 8

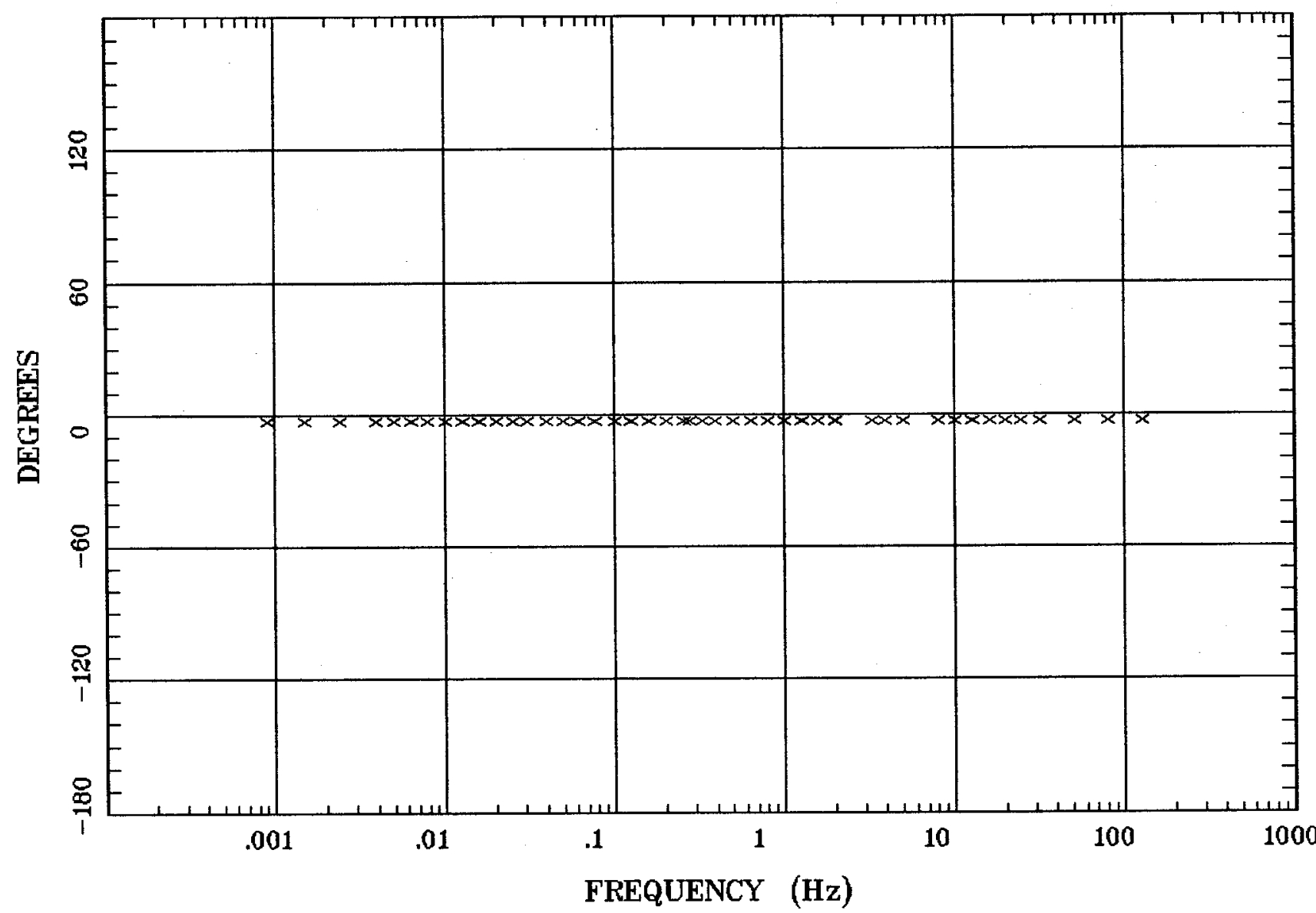

Client:

Remote:

Acquired: 19:5 Mar 08, 1998

Survey Co:
Rotation:

Filename: s8ed.avg

Channels: Ch1 Ch2 Ch3 ch4 Ch5 Ch3 Ch4

Plotted: 10:18 Dec 13, 2004

$<$ EMI - ElectroMagnetic Instruments 


\section{IMPEDANCE SKEW}

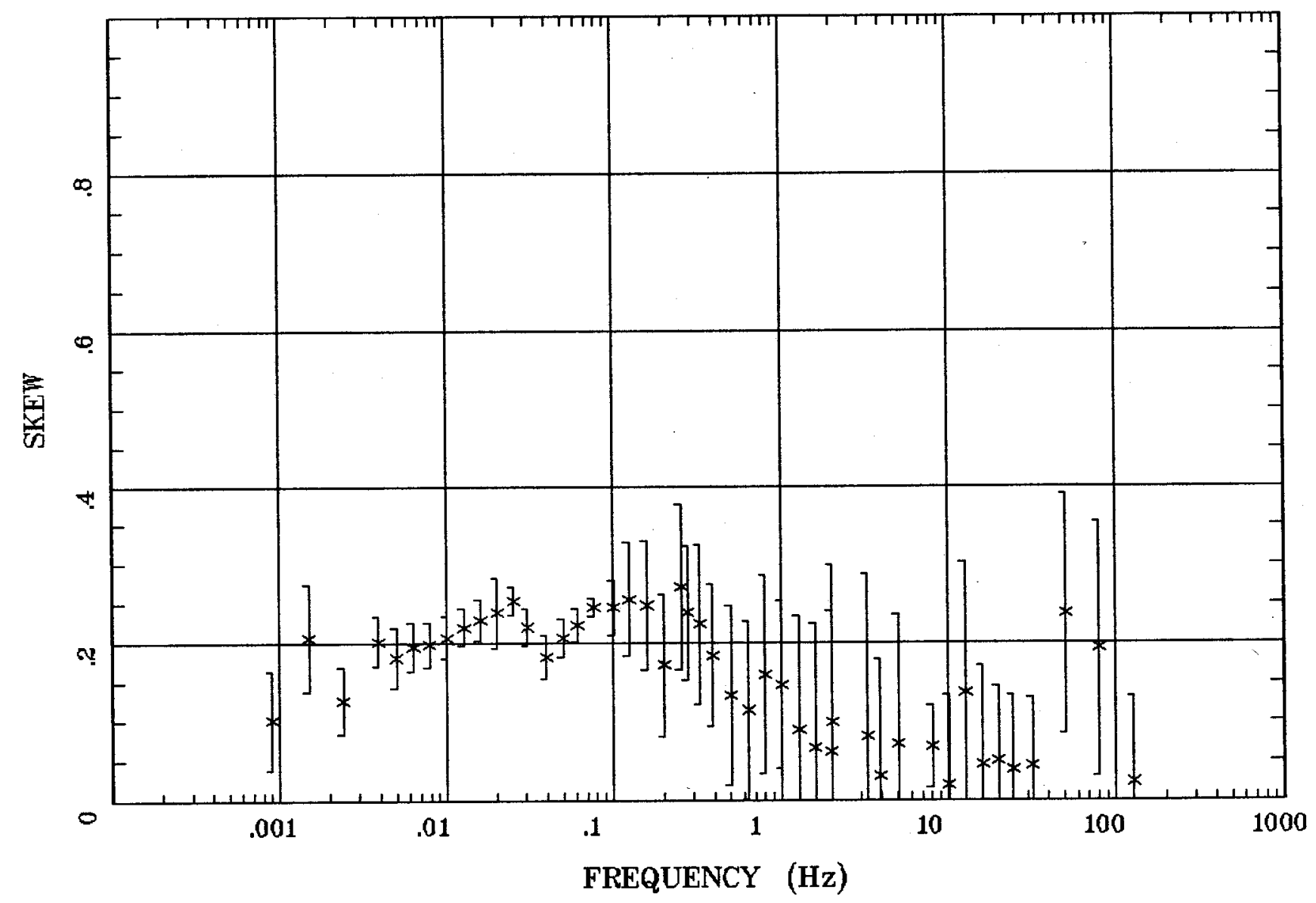

Client:

Remote:

Acquired: 19:5 Mar 08, 1998 Survey Co:
Rotation:

Filename: sBed.avg

Channels: Ch1 Ch2 Ch3 Ch4 Ch5 Ch3 Ch4 Plotted: 10:18 Dec 13, 2004

< EMI - ElectroMagnetic Instruments > 


\section{Station 8}

\section{E MULT Coh.}

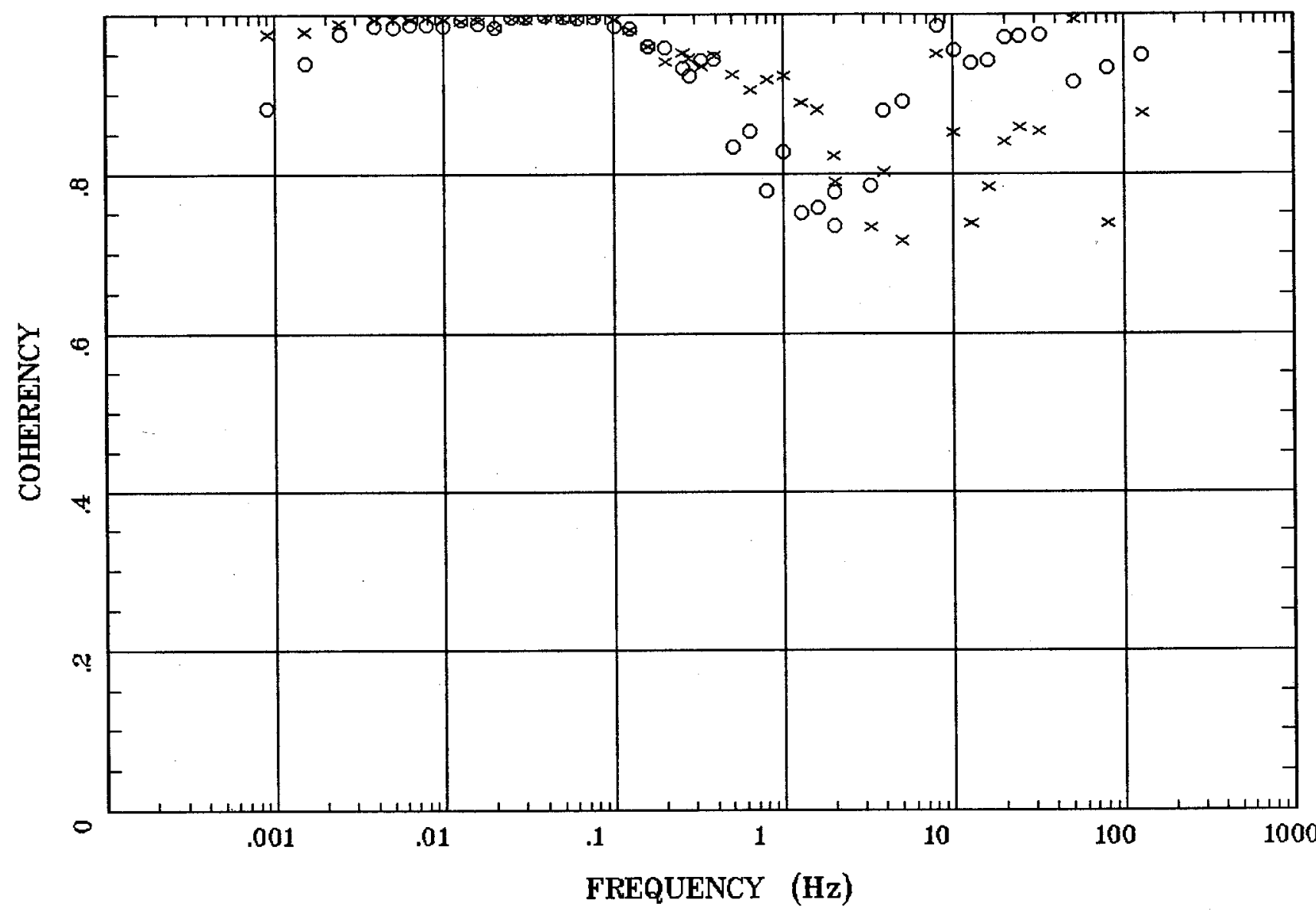

Client:

Remote:

Acquired: 19:5 Mar 08, 1998 Survey Co:
Rotation:

Filename: sBed.avg

Channels: Ch1 Ch2 Ch3 Ch4 Ch5 Ch3 Ch4 Plotted: 10:18 Dec 13, 2004

< EMI - ElectroMagnetic Instruments 


\section{Station 8}

\section{POLAR PLOTS}

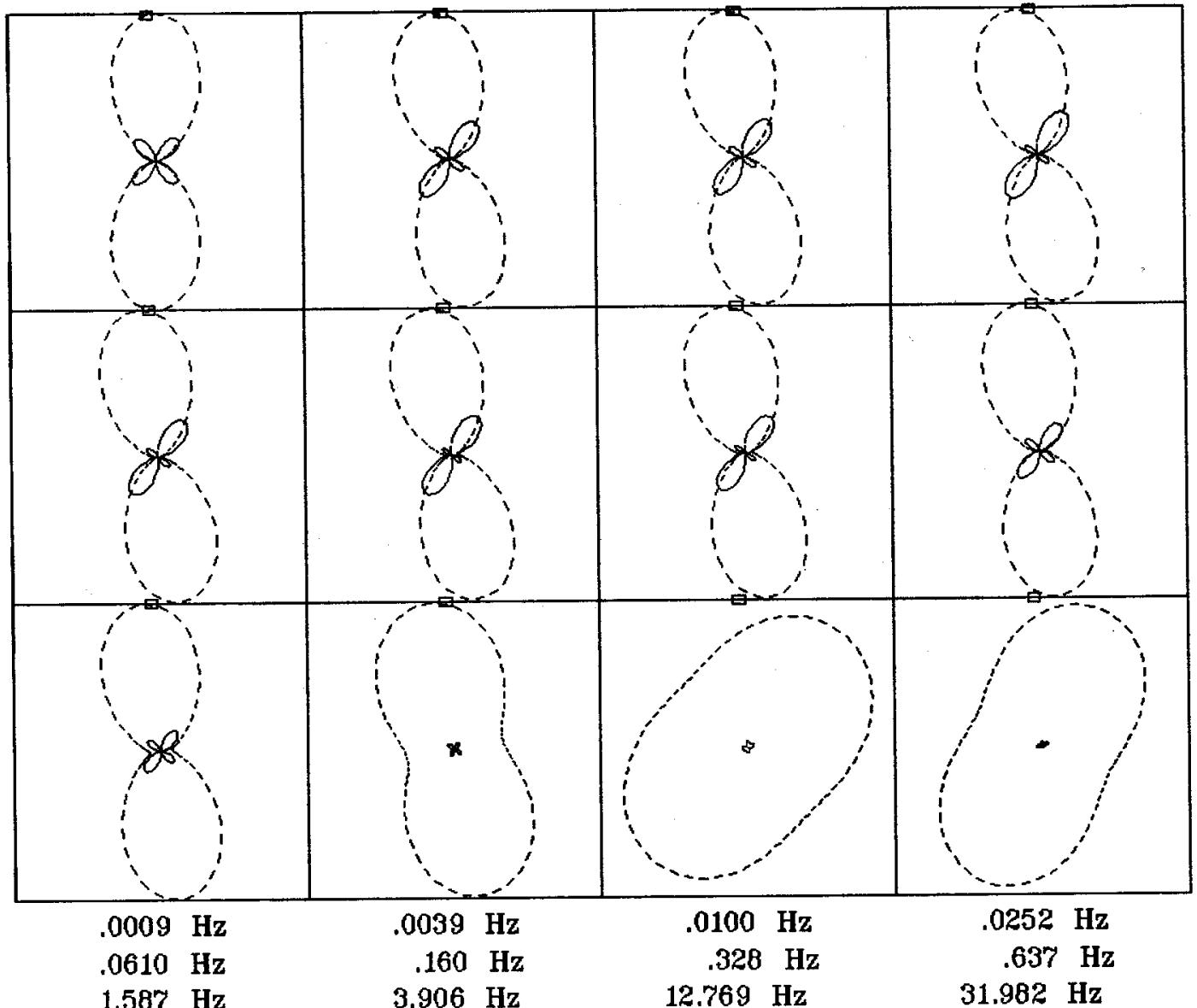

\section{Client:}

Remote:

Acquired: 19:5 Mar 08, 1998

Survey Co:
Rotation:

Filename: sbed.avg

Channels: Ch1 Ch2 Ch3 Ch4 Ch5 Ch3 Ch4

Plotted: 10:18 Dec 13, 2004

< EMI - ElectroMagnetic Instruments > 


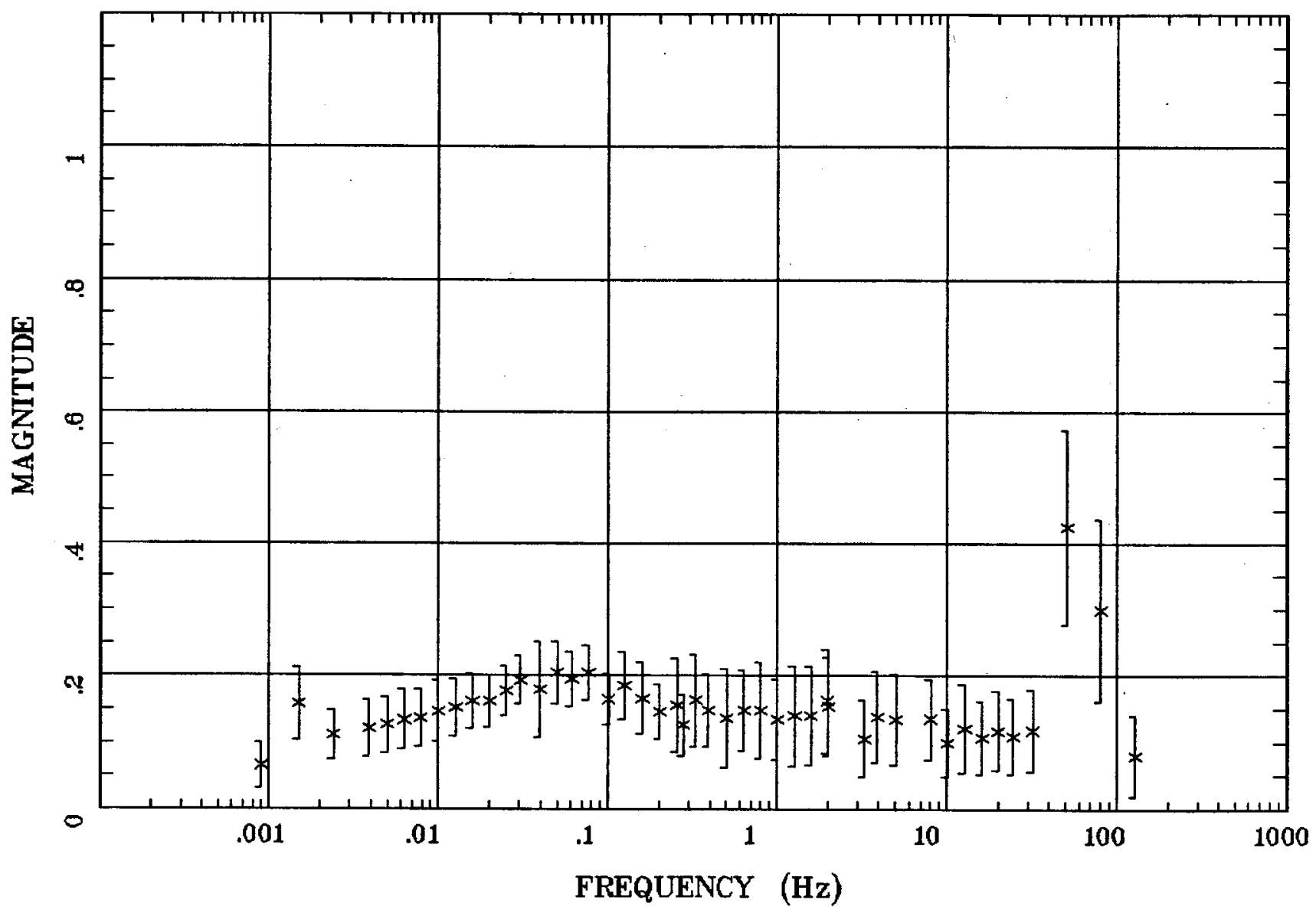

Client:

Remote:

Acquired: 19:5 Mar 08, 1998

Survey Co:
Rotation:

Filename: sbed.avg

Channels: Ch1 Ch2 Ch3 Ch4 Ch5 Ch3 Ch4

Plotted: 10:18 Dec 13, 2004

< EMI - ElectroMagnetic Instruments > 


\section{TIPPER STRIKE}

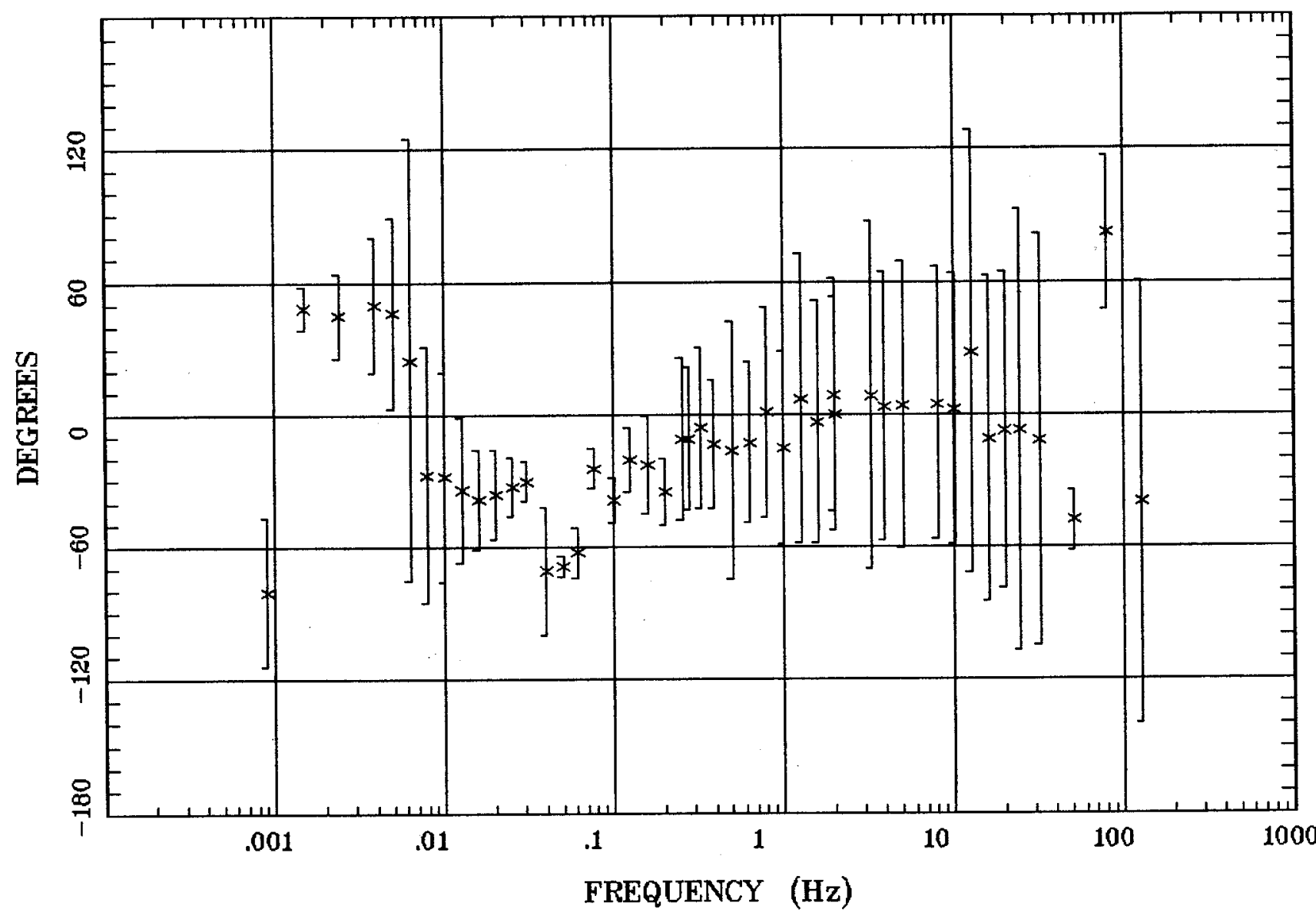

Client:

Remote:

Acquired: 19:5 Mar 08, 1998 Survey Co:
Rotation:

Filename: sbed.avg

Channels: Ch1 Ch2 Ch3 Ch4 Ch5 Ch3 Ch4 Plotted: 10:18 Dec 13, 2004

< EMI - ElectroMagnetic Instruments 


\section{HzHx.x Coh HzHy.o}

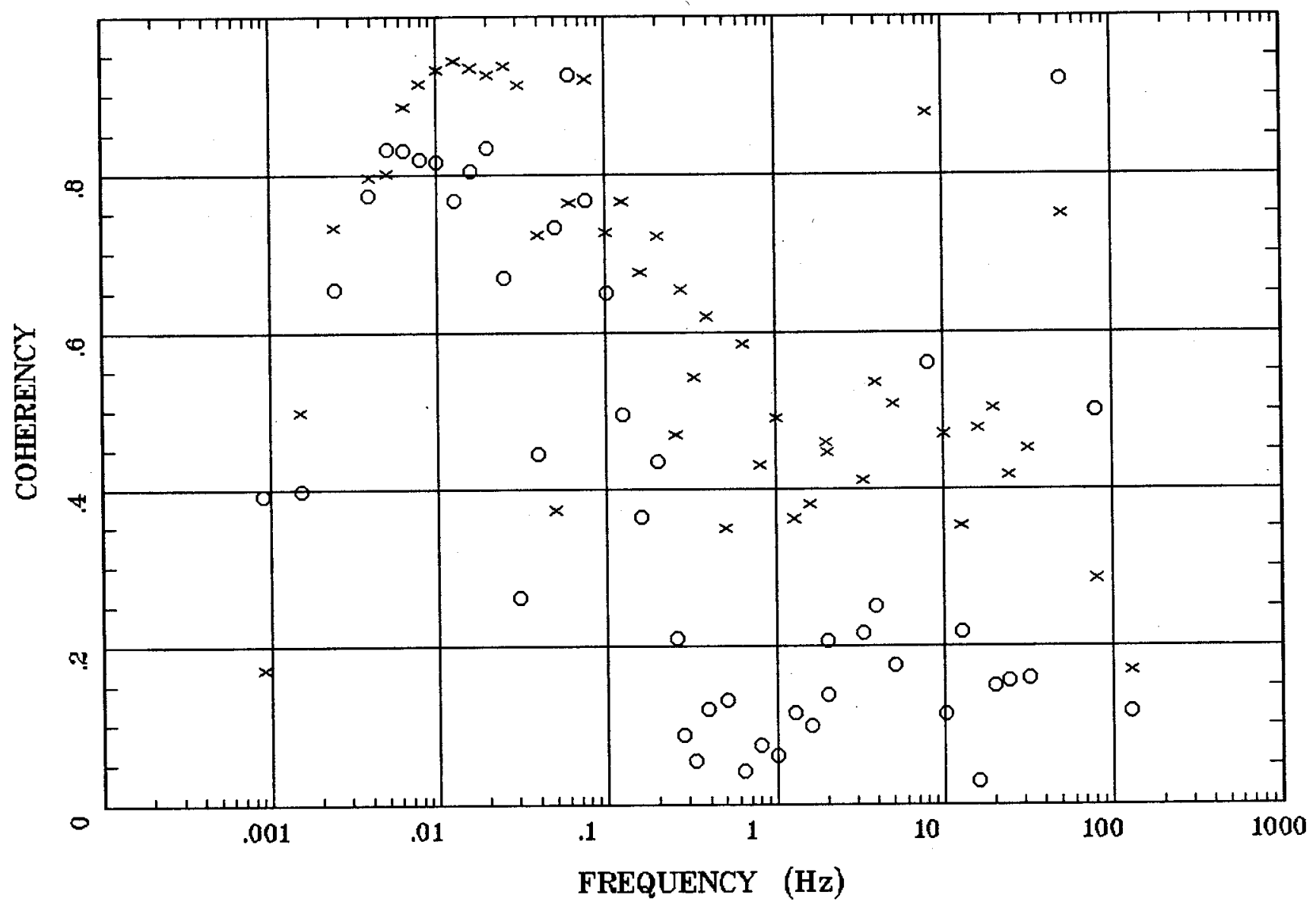

Client:

Remote:

Acquired: 19:5 Mar 08, 1998 Survey Co:

\section{Rotation:}

Filename: sbed.avg

Channels: Ch1 Ch2 Ch3 Ch4 Ch5 Ch3 Ch4 Plotted: 10:18 Dec 13, 2004

< EMI - ElectroMagnetic Instruments > 


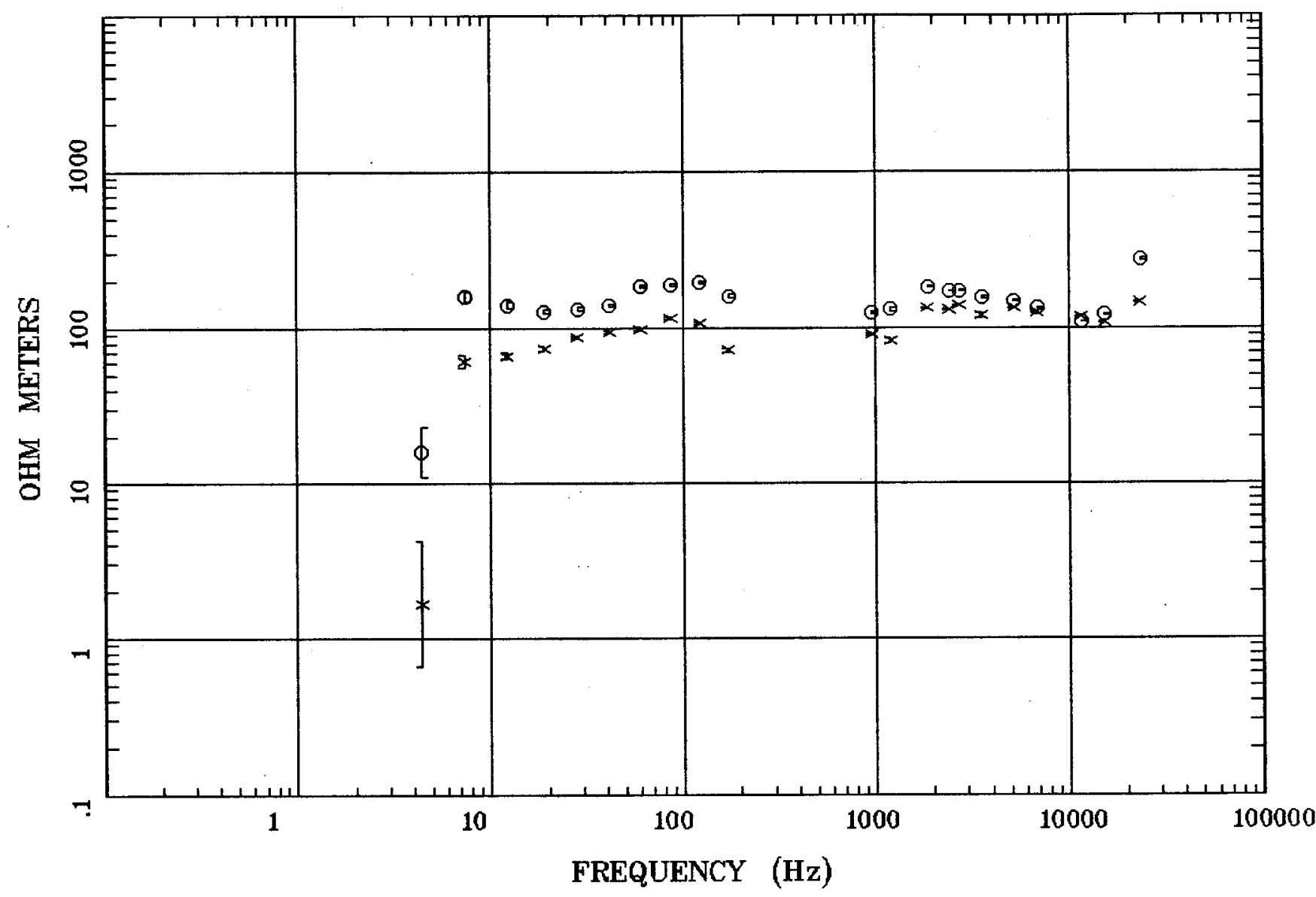

Client: Water Resources Remote: none Acquired: 13:5 Dec 07, 2003 Survey Co:USGS
Rotation:

Filename: nts1.avg

Channels: Ch1 Ch2 Ch3 ch4 Ch5 Ch3 Ch4 Plotted: 08:42 Dec 07, 2004

< EMI - ElectroMagnetic Instruments > 


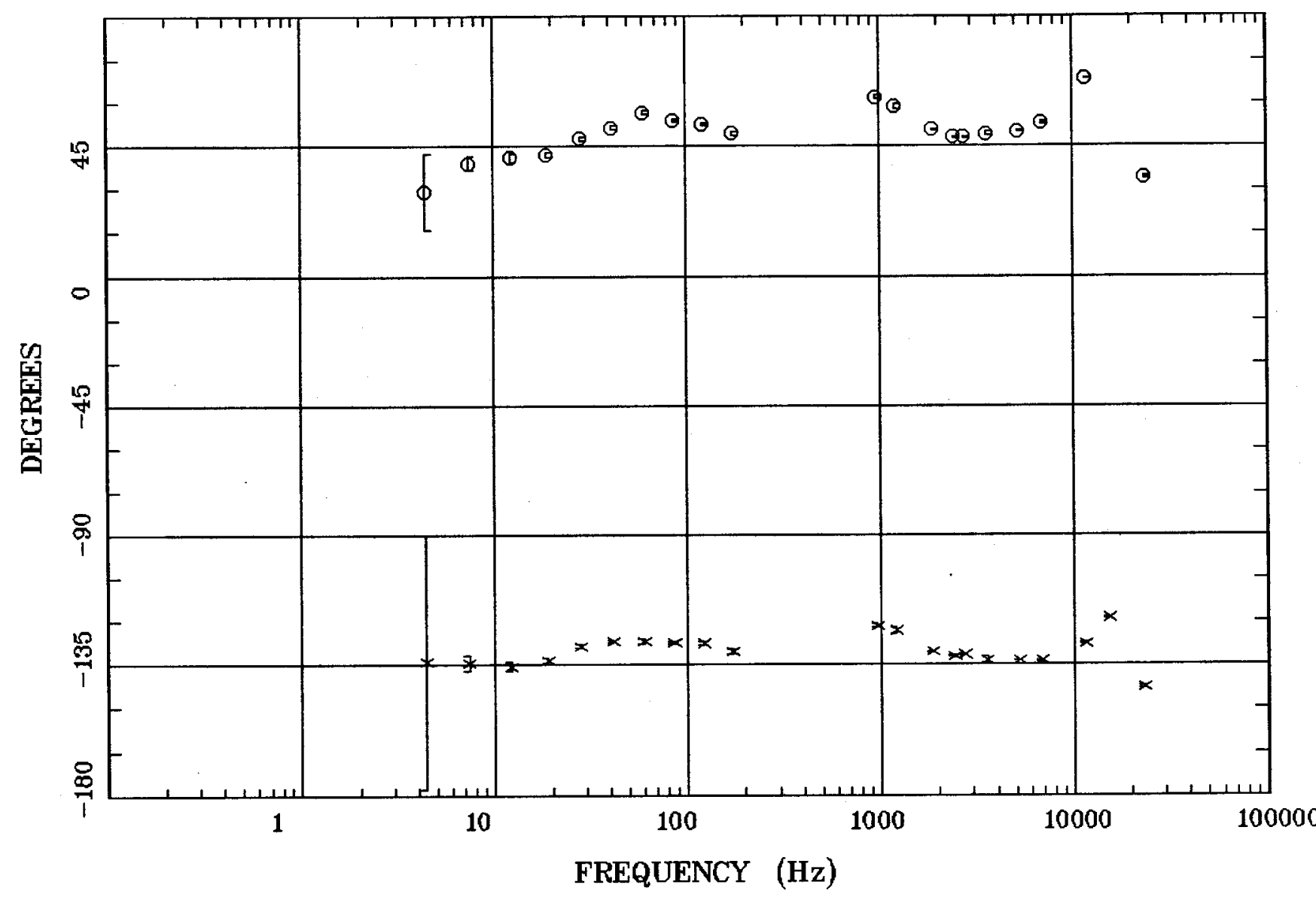

Client: Water Resources

Remote: none

Acquired: 13:5 Dec 07, 2003

Survey Co:USGS
Rotation:

Filename: nts1.avg

Channels: Ch1 Ch2 Ch3 Ch4 Ch5 Ch3 Ch4 Plotted: 08:42 Dec 07, 2004

< EMI - ElectroMagnetic Instruments 


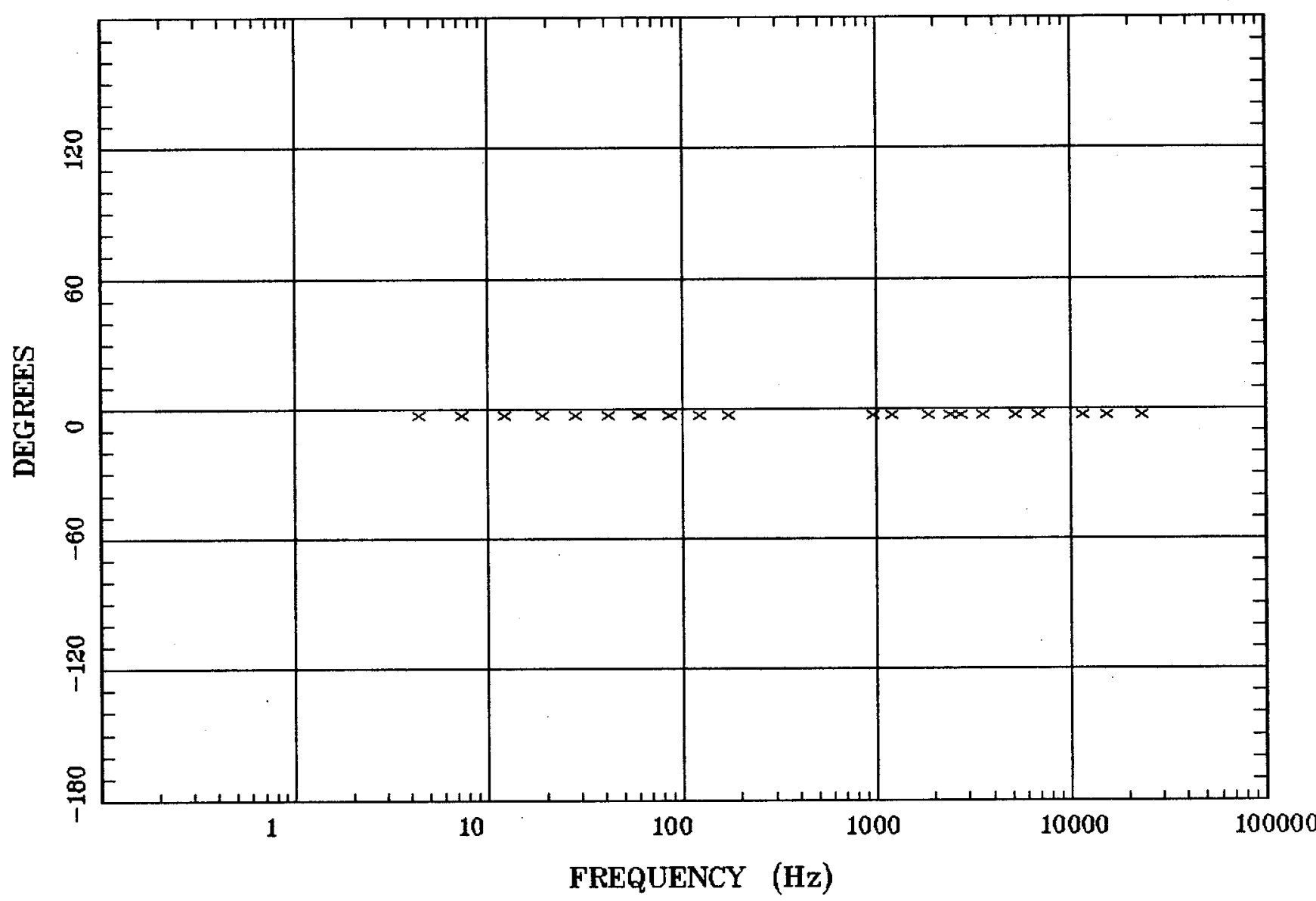

Client: Water Resources Remote: none Acquired: 13:5 Dec 07, 2003 Survey Co:USGS
Rotation:

Filename: nts1.avg

Channels: Ch1 Ch2 Ch3 Ch4 Ch5 Ch3 Ch4 Plotted: 08:42 Dec 07, 2004

< EMI - ElectroMagnetic Instruments > 


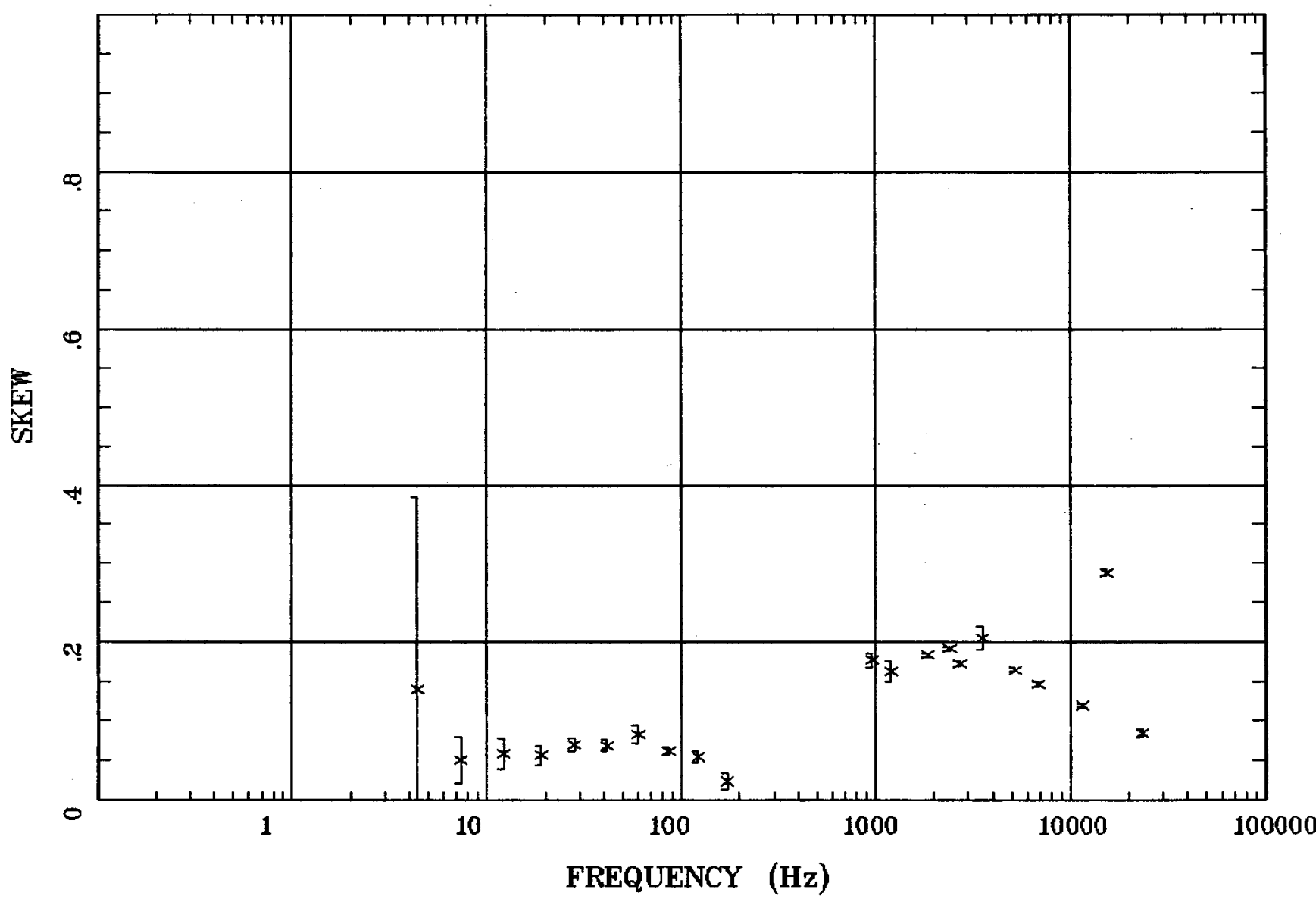

Client: Water Resources Remote: none Acquired: 13:5 Dec 07, 2003 Survey Co:USGS
Rotation:

Filename: nts1.avg

Channels: Ch1 Ch2 Ch3 Ch4 Ch5 Ch3 Ch4 Plotted: 08:42 Dec 07, 2004

< EMI - ElectroMagnetic Instruments > 


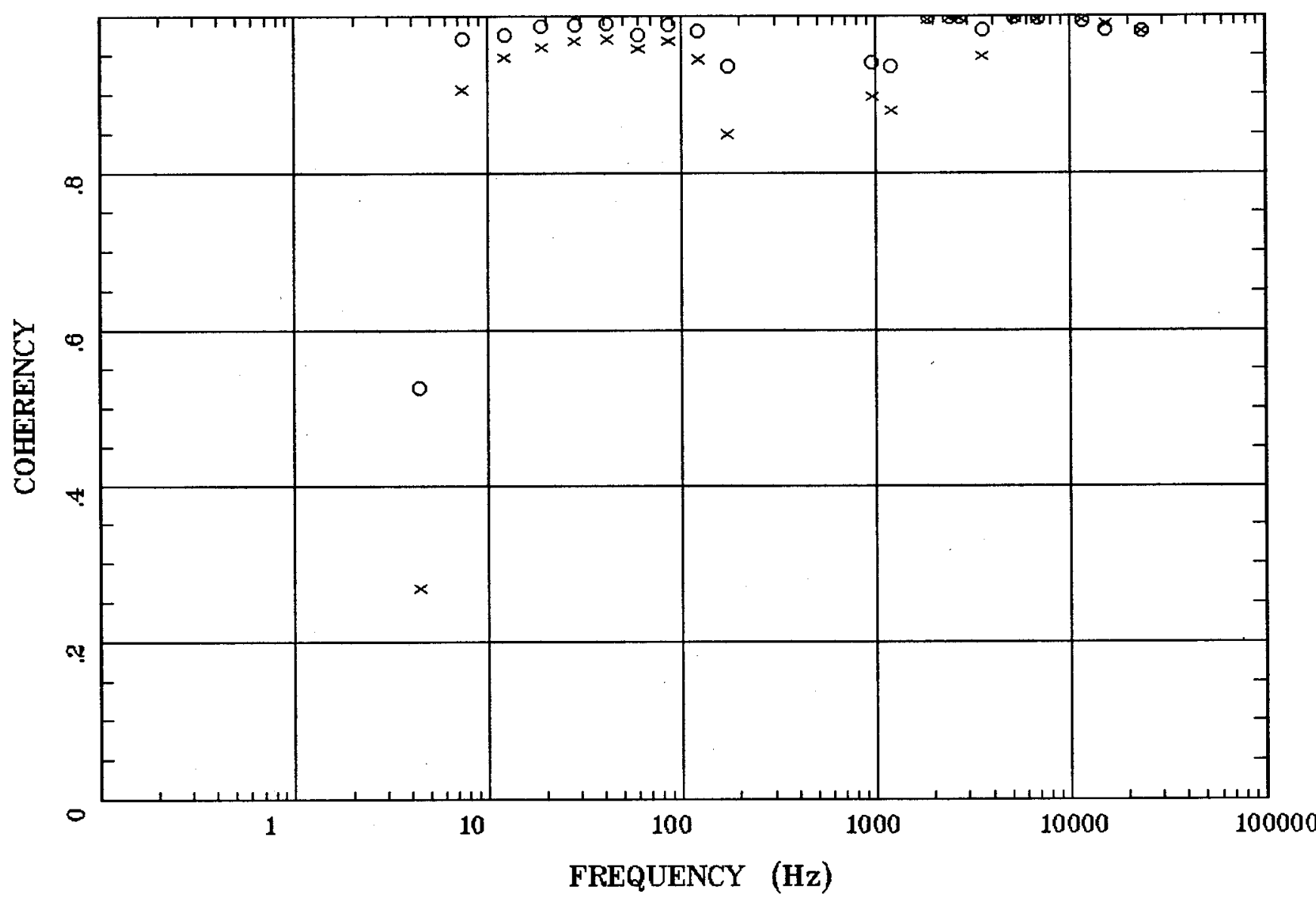

Client: Fater Resources

Remote: none

Acquired: 13:5 Dec 07, 2003

Survey Co:USGS
Rotation:

Filename: nts1.avg

Channels: Ch1 Ch2 Ch3 Ch4 Ch5 Ch3 Ch4

Plotted: 08:42 Dec 07, 2004

$<$ EMI - ElectroMagnetic Instruments 


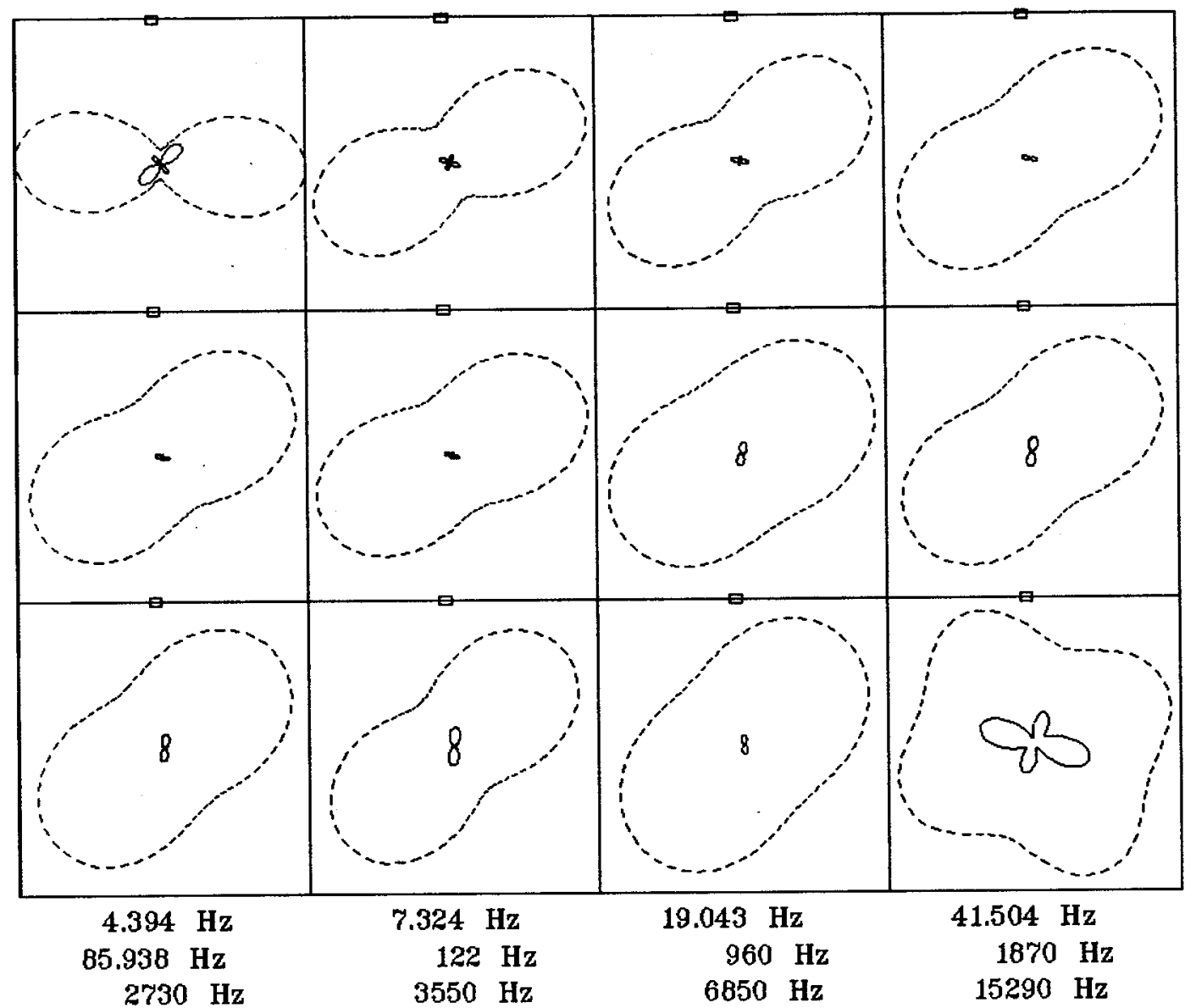

Client: Water Resources

Rotation:

Filename: ntsl.avg

Remote: none

Channels: Ch1 Ch2 Ch3 Ch4 Ch5 Ch3 Ch4

Acquired: 13:5 Dec 07, 2003

Plotted: 08:42 Dec 07, 2004

Survey Co:USGS

< EMI - ElectroMagnetic Instruments 


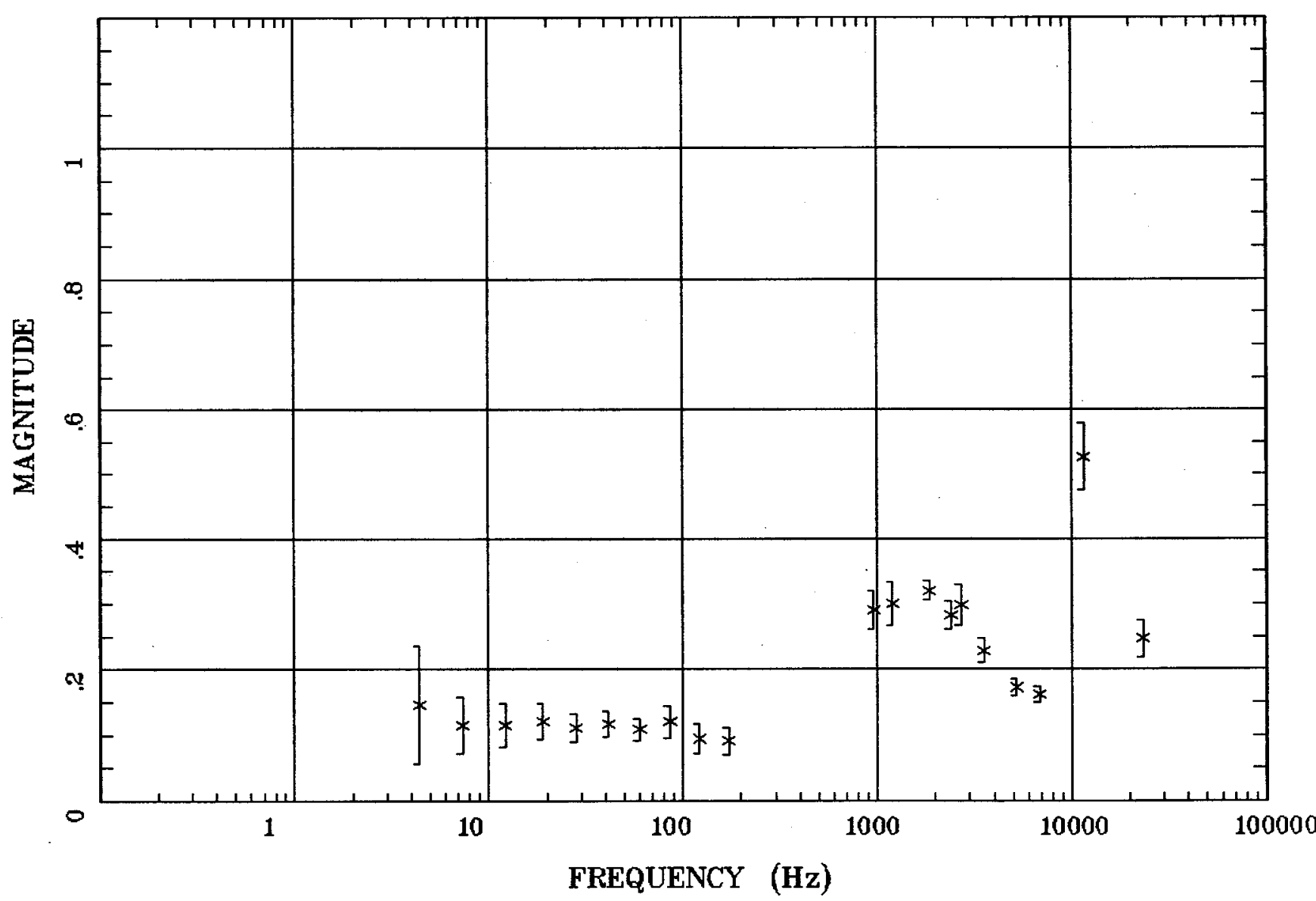

Client: Fater Resources Remote: none Acquired: 13:5 Dec 07, 2003 Survey Co:USGS
Rotation:

Filename: nts1.avg

Channels: Ch1 Ch2 Ch3 Ch4 Ch5 Ch3 Ch4 Plotted: 08:42 Dec 07, 2004

$<$ EMI - ElectroMagnetic Instruments 


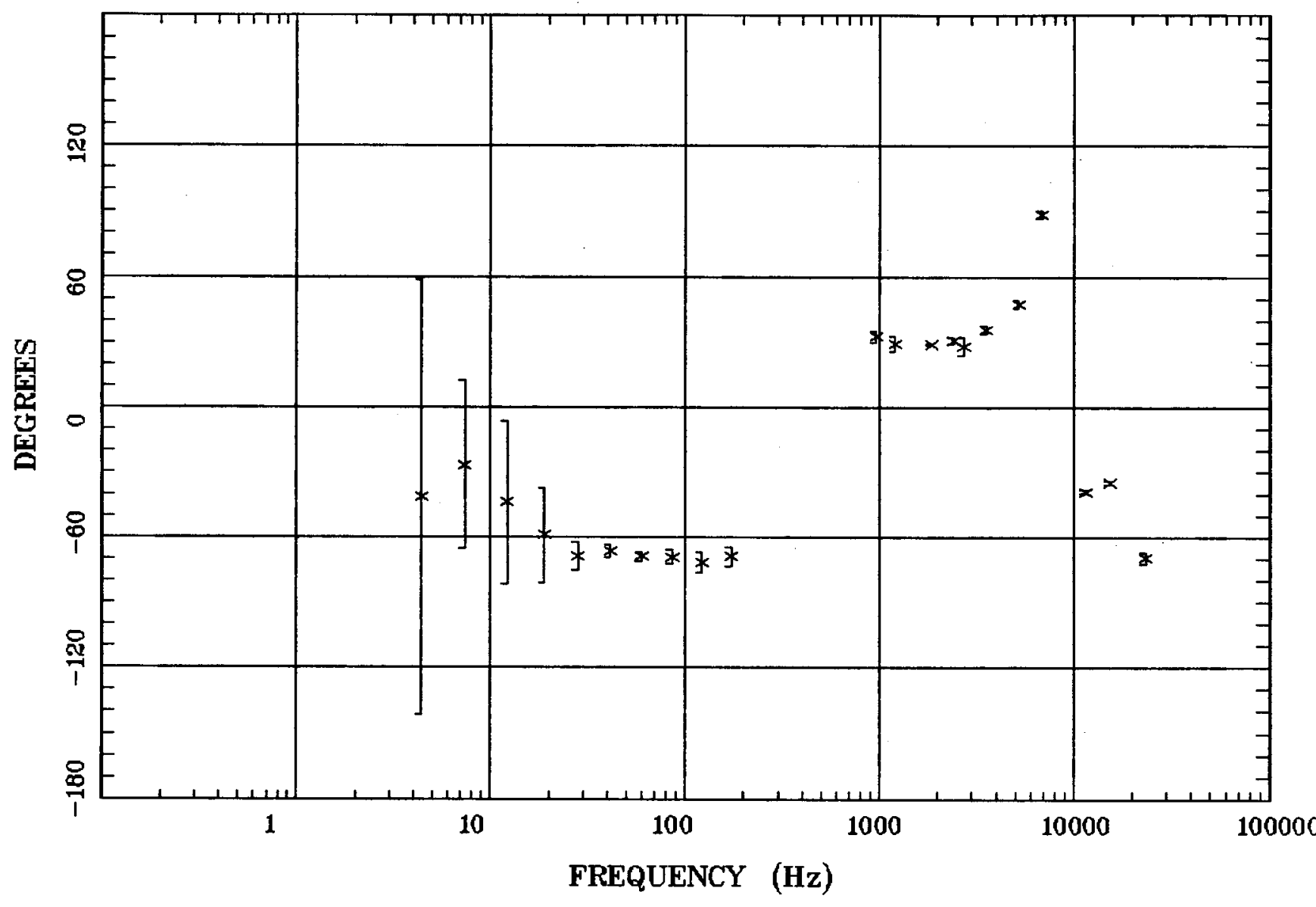

Client: Water Resources

Remote: none

Acquired: 13:5 Dec 07, 2003

Survey Co:USGS
Rotation:

Filename: nts1.avg

Channels: Ch1 Ch2 Ch3 Ch4 Ch5 Ch3 Ch4 Plotted: 08:42 Dec 07, 2004

< EMI - ElectroMagnetic Instruments 


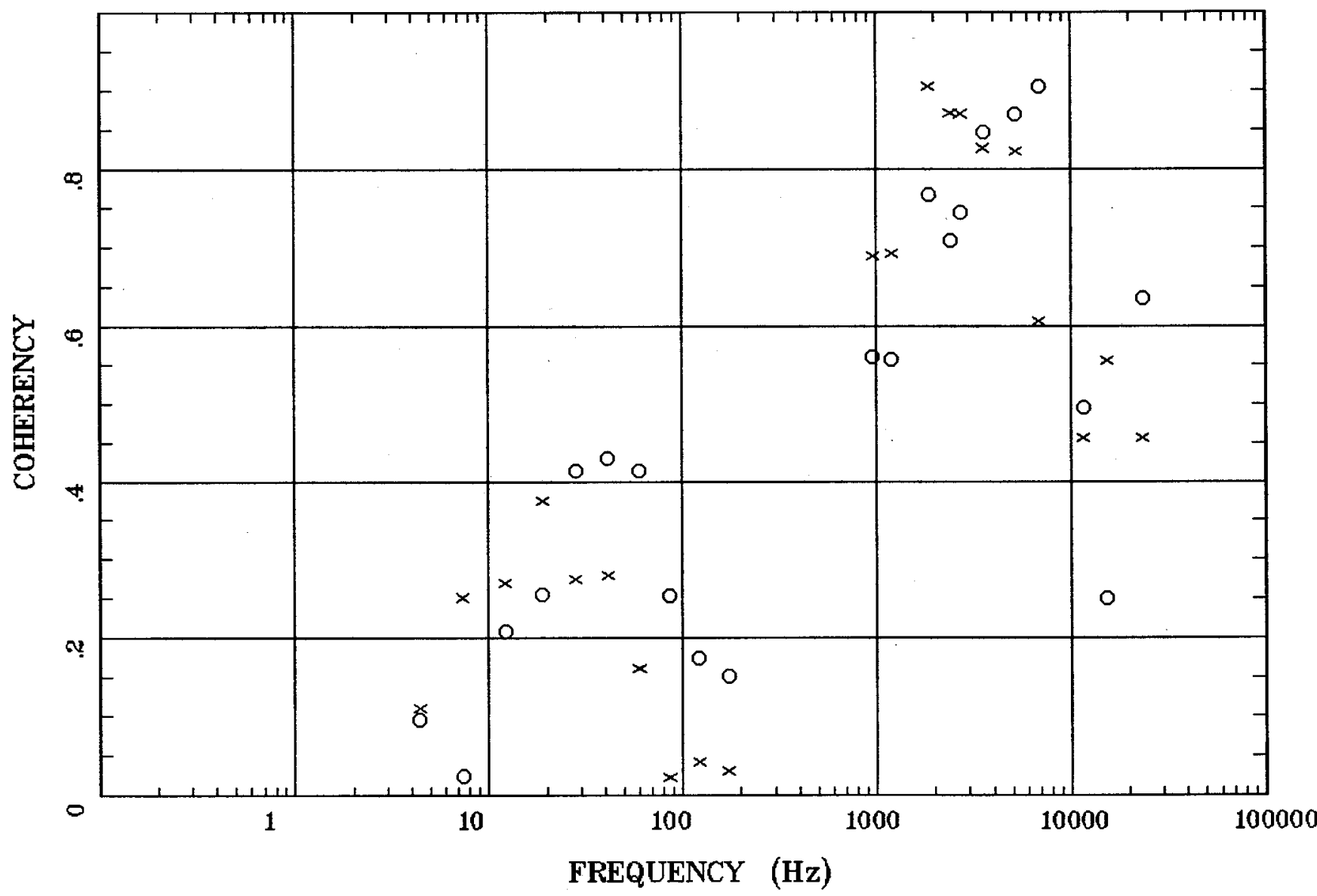

Client: Water Resources Remote: none Acquired: 13:5 Dec 07, 2003 Survey Co:USGS
Rotation:

Filename: nts1.avg

Channels: Ch1 Ch2 Ch3 Ch4 Ch5 Ch3 Ch4 Plotted: 08:42 Dec 07, 2004

$<$ EMI - ElectroMagnetic Instruments 


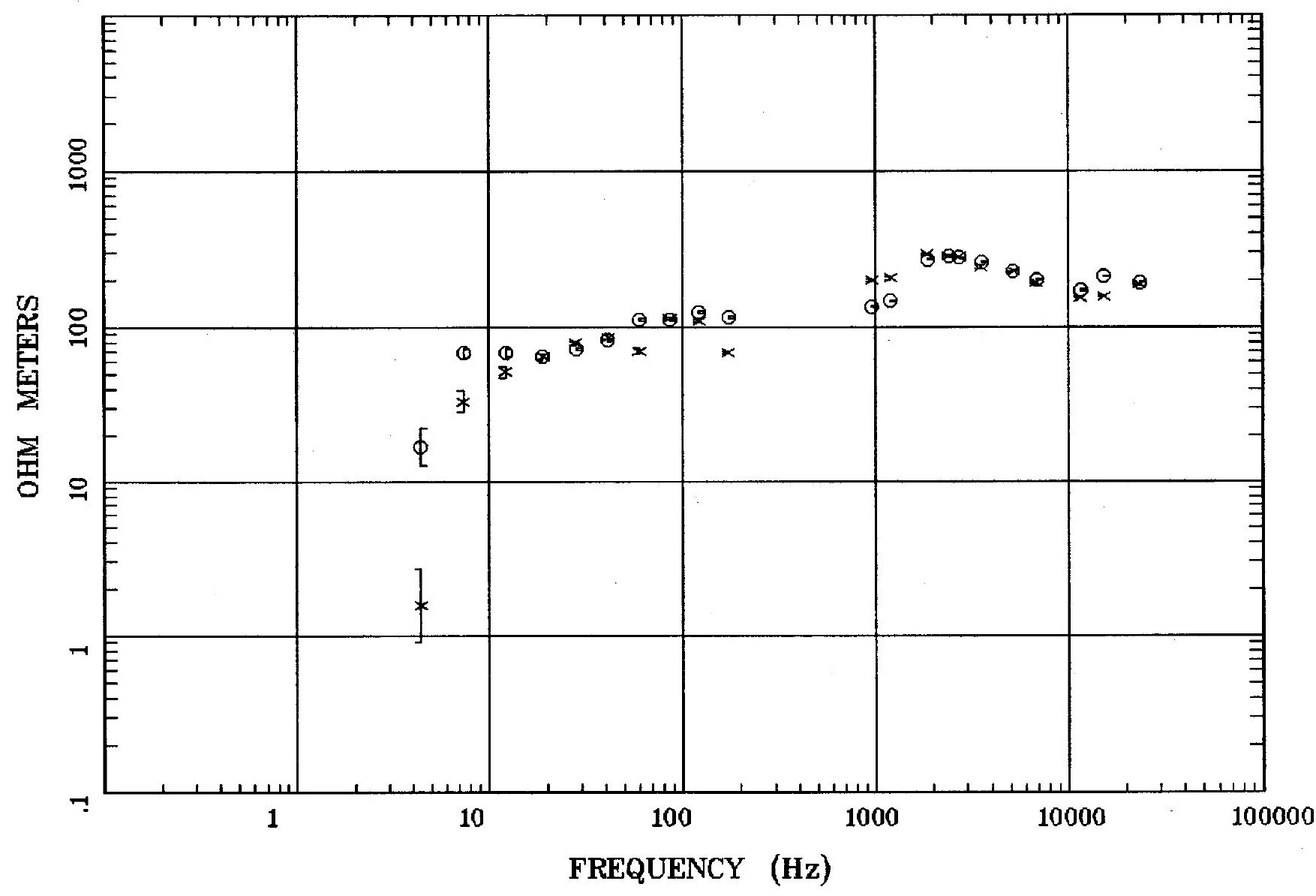

Client: Water Resources Remote: none Acquired: 11:2 Dec 07, 2003 Survey Co:USGS
Rotation:

Filename: nts2.avg

Channels: Ch1 Ch2 Ch3 Ch4 Ch5 Ch3 Ch4 Plotted: 08:43 Dec 07, 2004

< EMI - ElectroMagnetic Instruments 


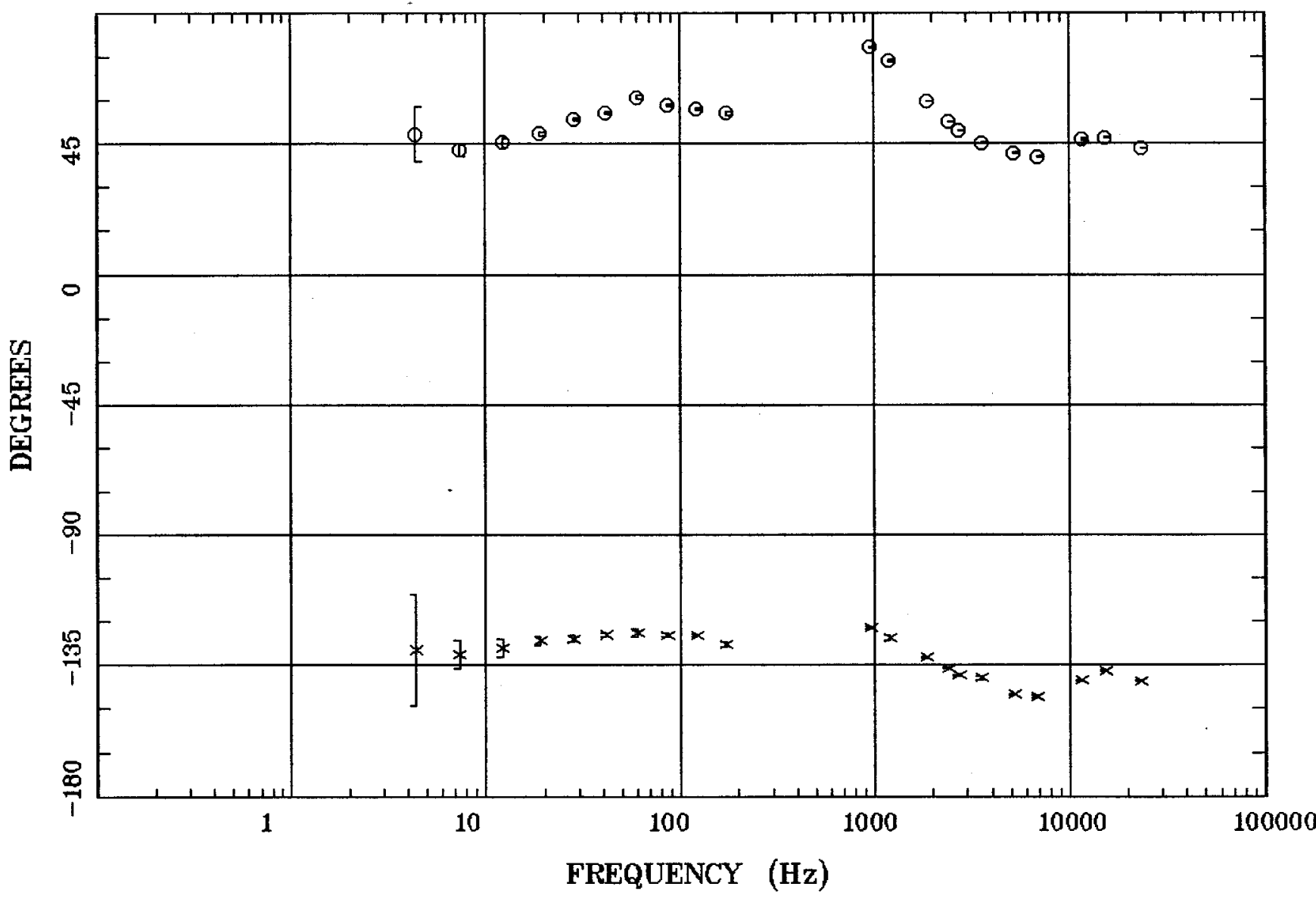

Client: Water Resources Remote: none Acquired: 11:2 Dec 07, 2003 Survey Co:USGS
Rotation:

Filename: nts2.avg

Channels: Ch1 Ch2 Ch3 Ch4 Ch5 Ch3 Ch4 Plotted: 08:43 Dec 07, 2004

< EMI - ElectroMagnetic Instruments > 


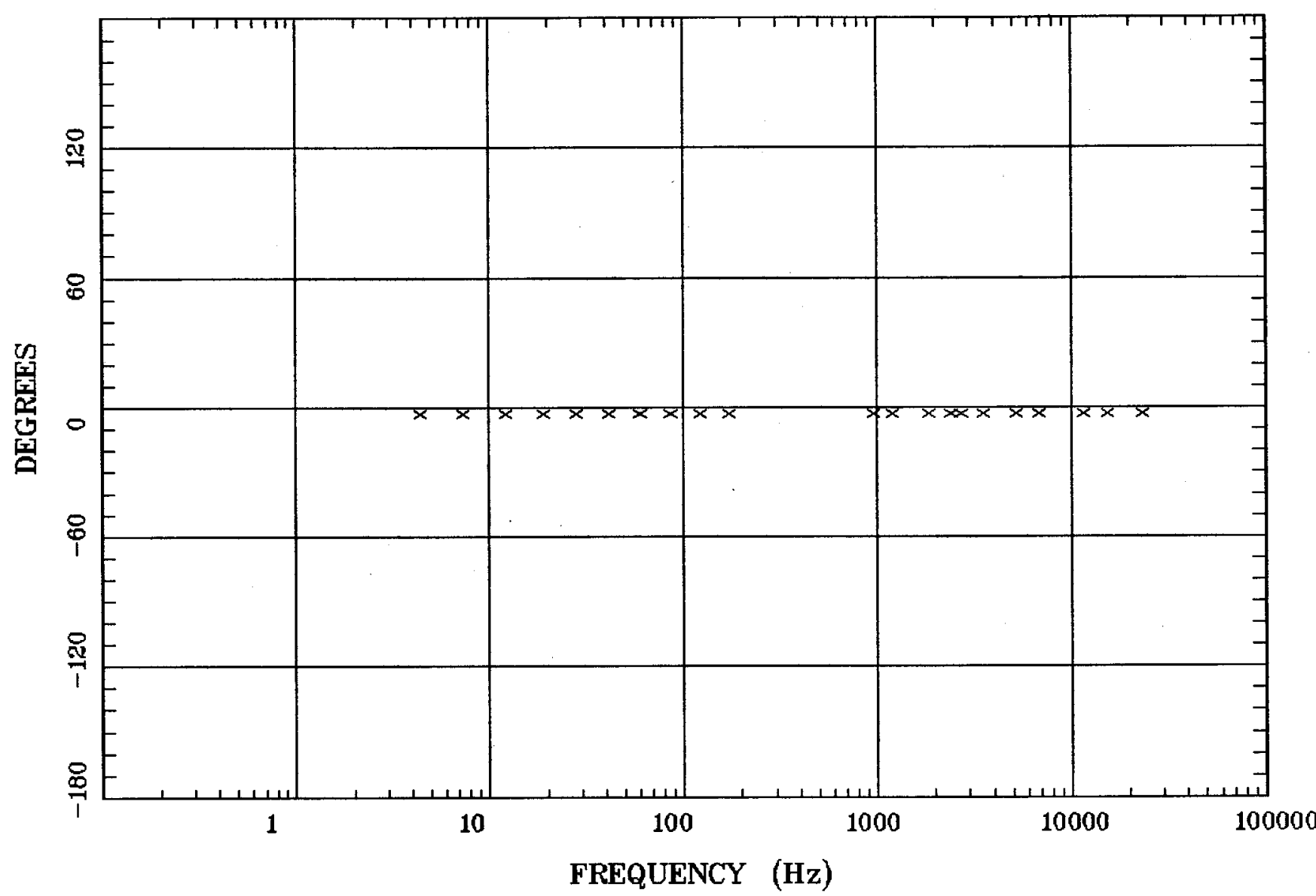

Client: Water Resources Remote: none Acquired: 11:2 Dec 07, 2003 Survey Ca:USGS
Rotation:

Filename: nts2.avg

Channels: Ch1 Ch2 Ch3 Ch4 Ch5 Ch3 Ch4 Plotted: 08:43 Dec 07, 2004

< EMI - ElectroMagnetic Instruments 


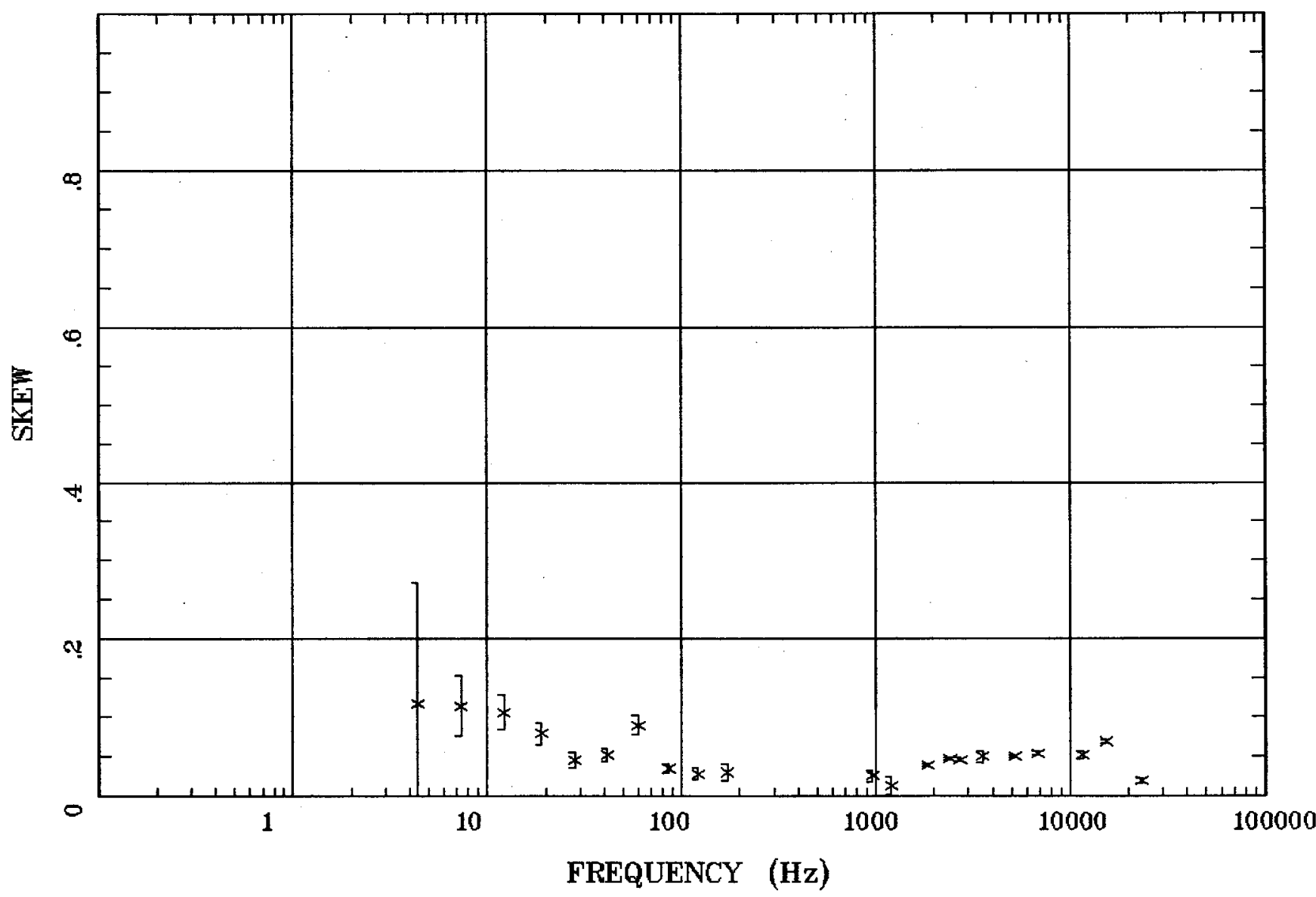

Client: Water Resources Remote: none Acquired: 11:2 Dec 07, 2003 Survey Co:USGS
Rotation:

Filename: nts2.avg

Channels: Ch1 Ch2 Ch3 Ch4 Ch5 Ch3 Ch4 Plotted: 08:43 Dec 07, 2004

< EMI - ElectroMagnetic Instruments 


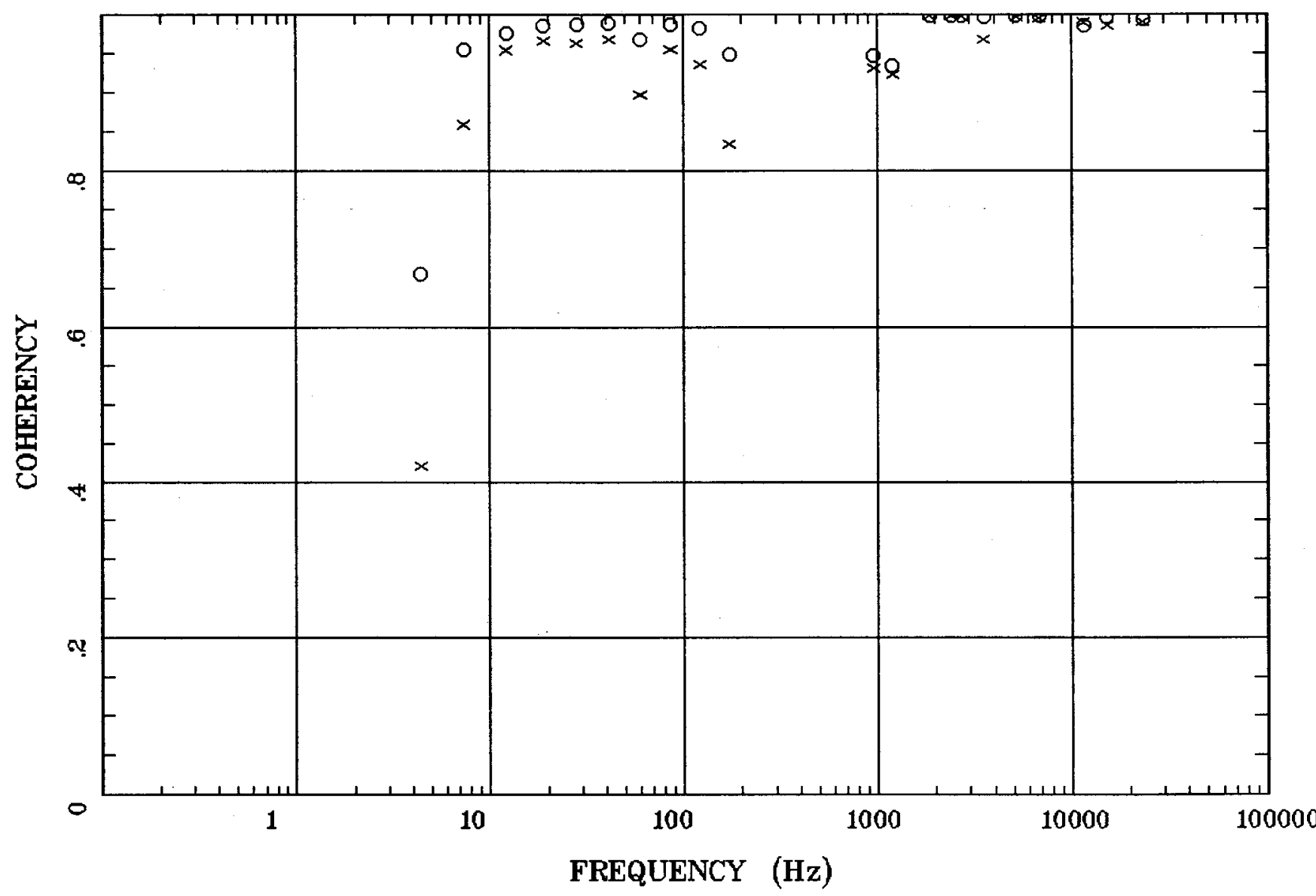

Client: Water Resources

Remote: none

Acquired: 11:2 Dec 07, 2003

Survey Co:USGS
Rotation:

Filename: nts2.avg

Channels: Ch1 Ch2 Ch3 Ch4 Ch5 Ch3 Ch4

Plotted: 08:43 Dec 07, 2004

< EMI - ElectroMagnetic Instruments > 


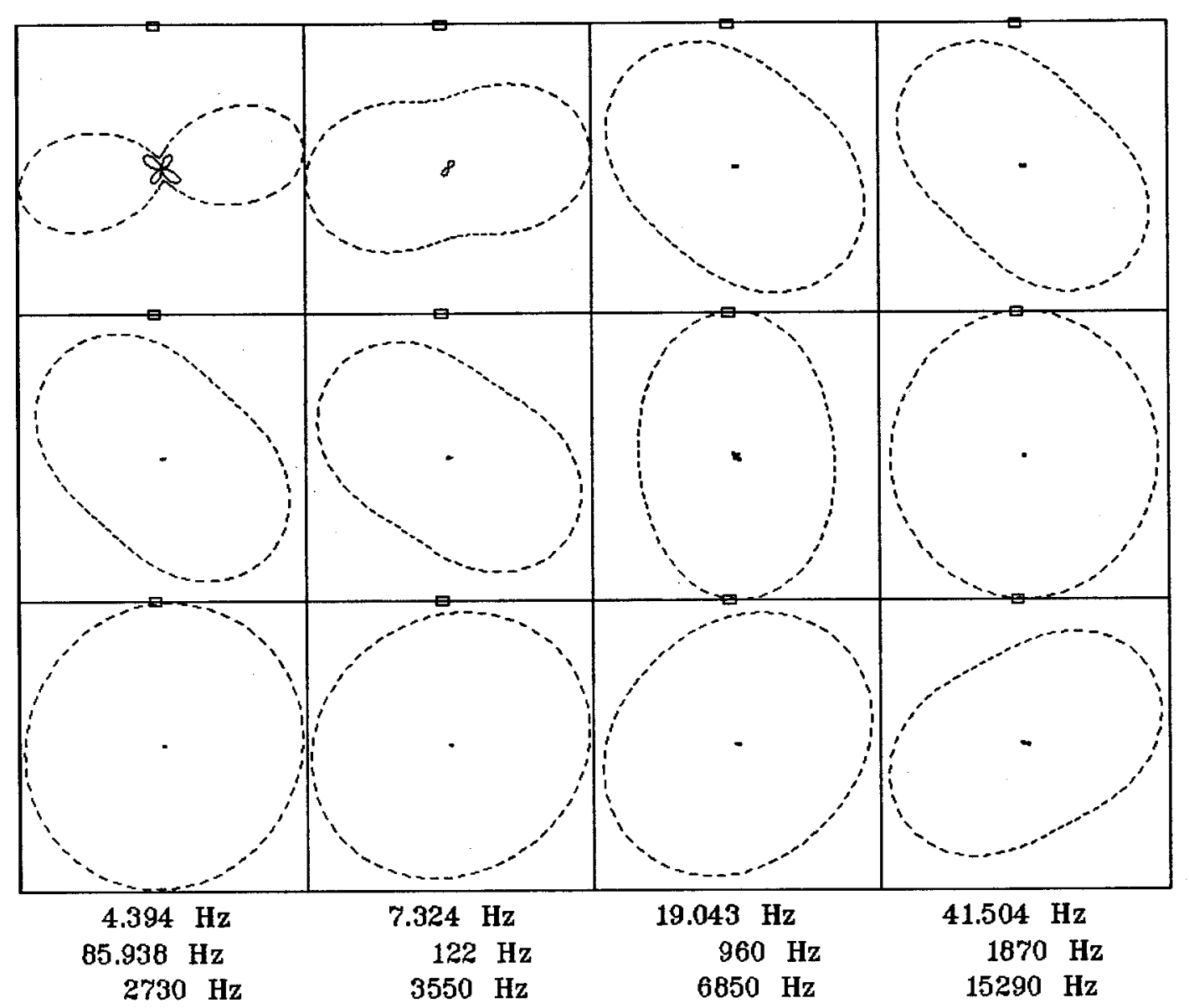

Client: Water Resources

Rotation:

Remote: none

Acquired: 11:2 Dec 07, 2003 Survey Co:USGS
Filename: nts2.avg

Channels: Ch1 Ch2 Ch3 Ch4 Ch5 Ch3 Ch4 Plotted: 08:43 Dec 07, 2004

< EMI - ElectroMagnetic Instruments 


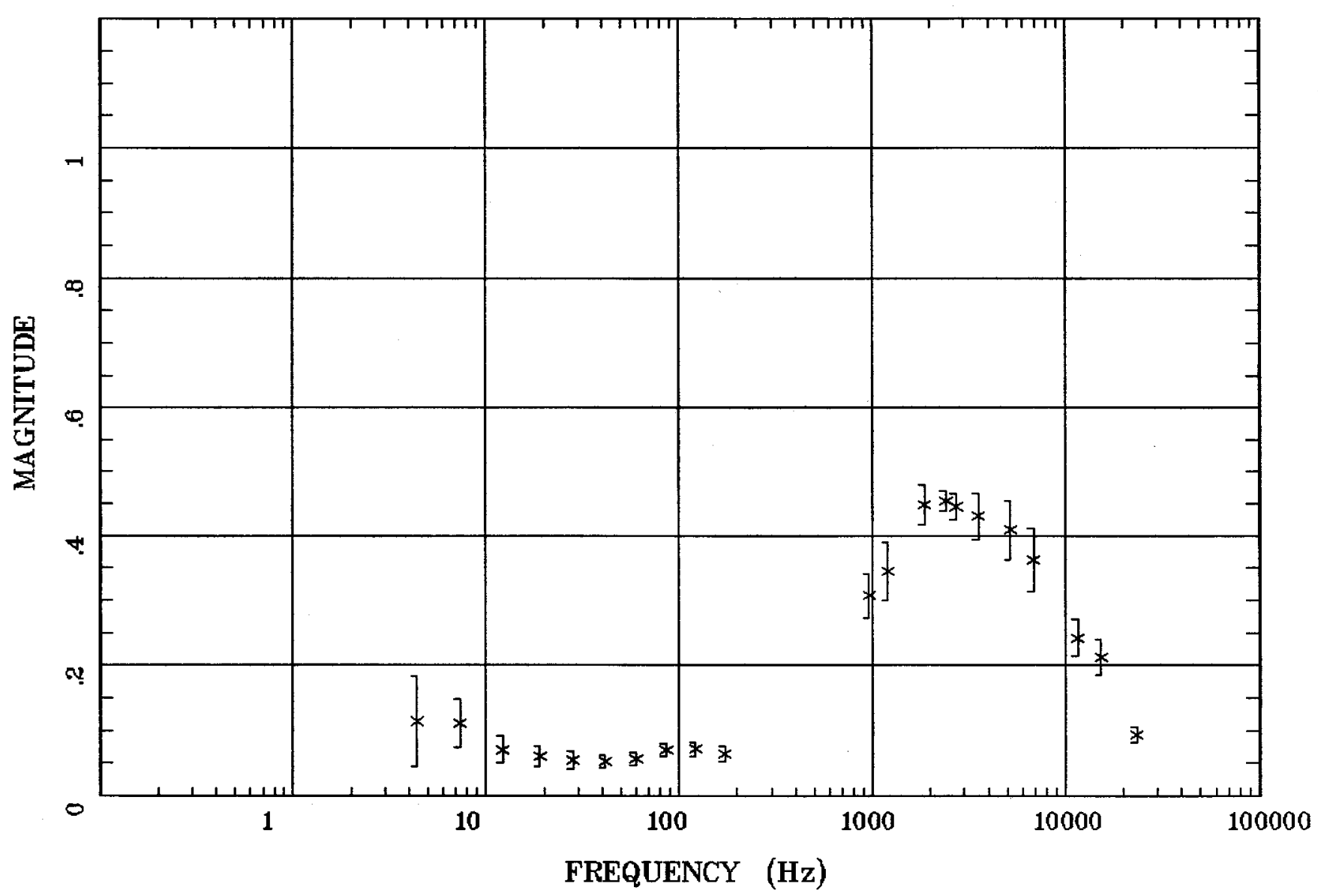

Client: Water Resources

Remote: none

Acquired: 11:2 Dec 07, 2003

Survey Co:USGS

\section{Rotation:}

Filename: nts2.avg

Channels: Ch1 Ch2 Ch3 Ch4 Ch5 Ch3 Ch4

Plotted: 08:43 Dec 07, 2004

< EMI - ElectroMagnetic Instruments > 


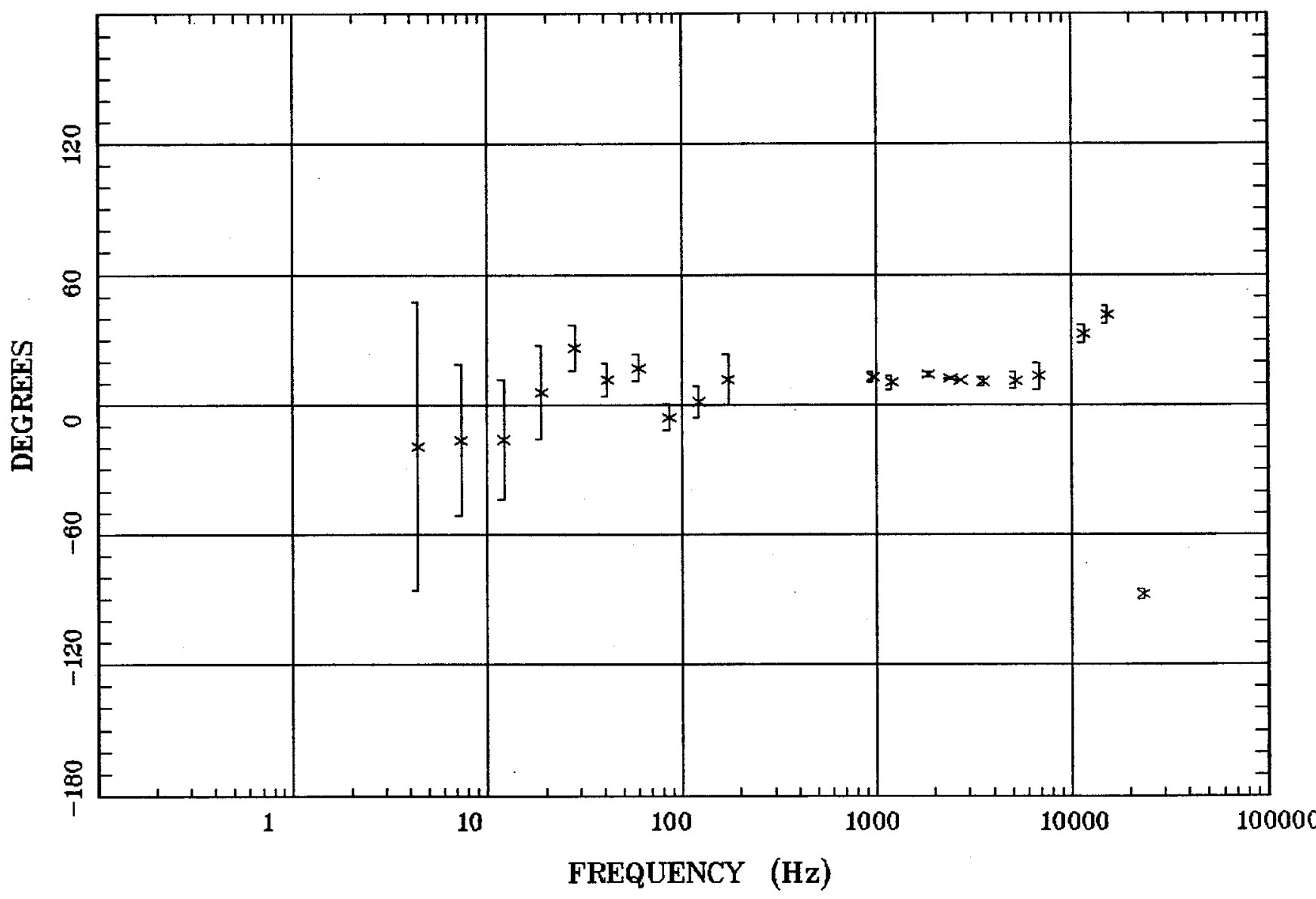

Client: Fater Resources Remote: none Acquired: 11:2 Dec 07, 2003 Survey Co:USGS
Rotation:

Filename: nts2.avg

Channels: Ch1 Ch2 Ch3 Ch4 Ch5 Ch3 Ch4 Plotted: 08:43 Dec 07, 2004

< EMI - ElectroMagnetic Instruments > 


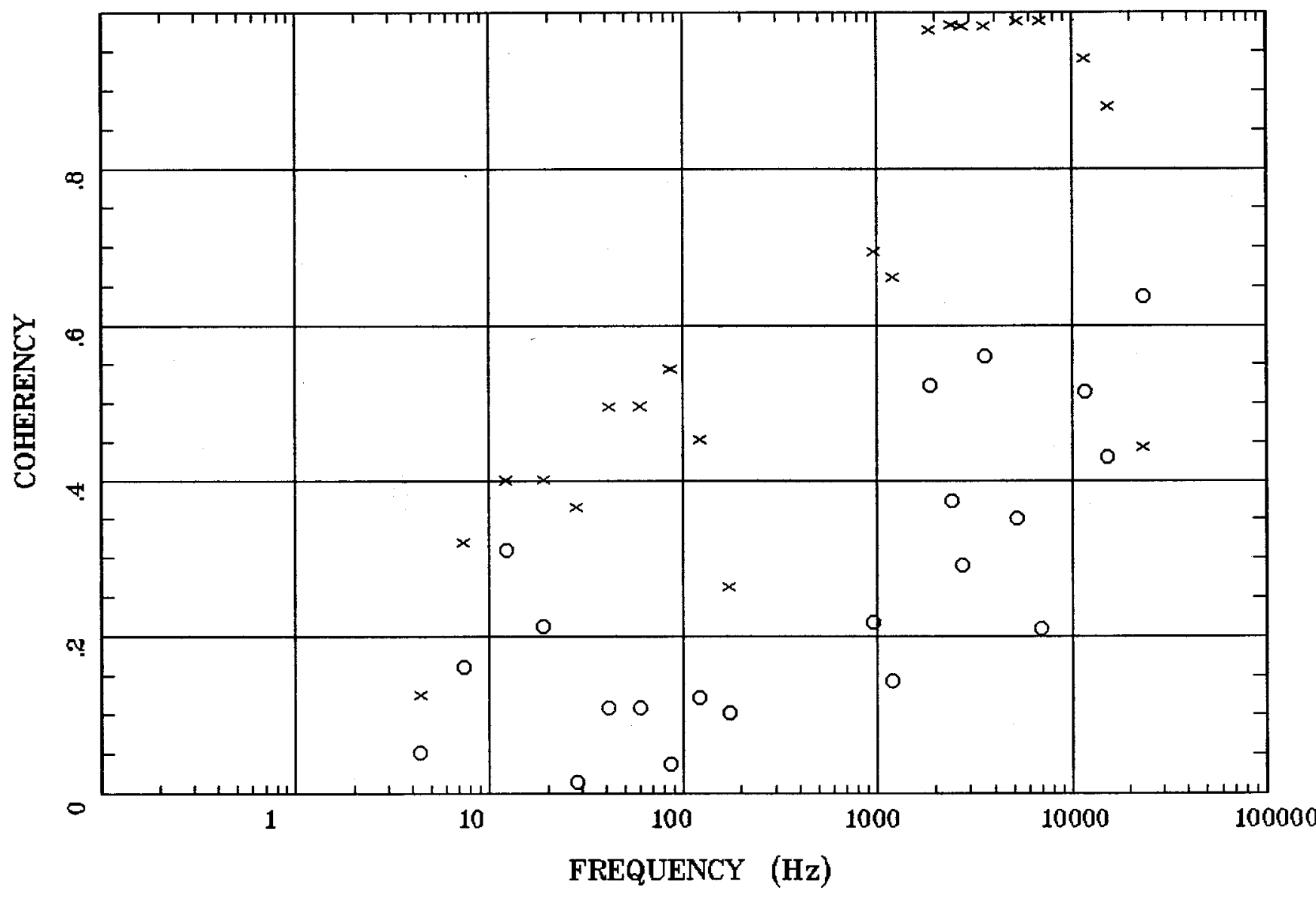

Client: Water Resources Remote: none Acquired: 11:2 Dec 07, 2003 Survey Co:USGS
Rotation:

Filename: nts2.avg

Channels: Ch1 Ch2 Ch3 Ch4 Ch5 Ch3 Ch4 Plotted: 08:43 Dec 07, 2004

< EMI - ElectroMagnetic Instruments > 


\section{APPARENT RESISTIVITY}

Priority 6

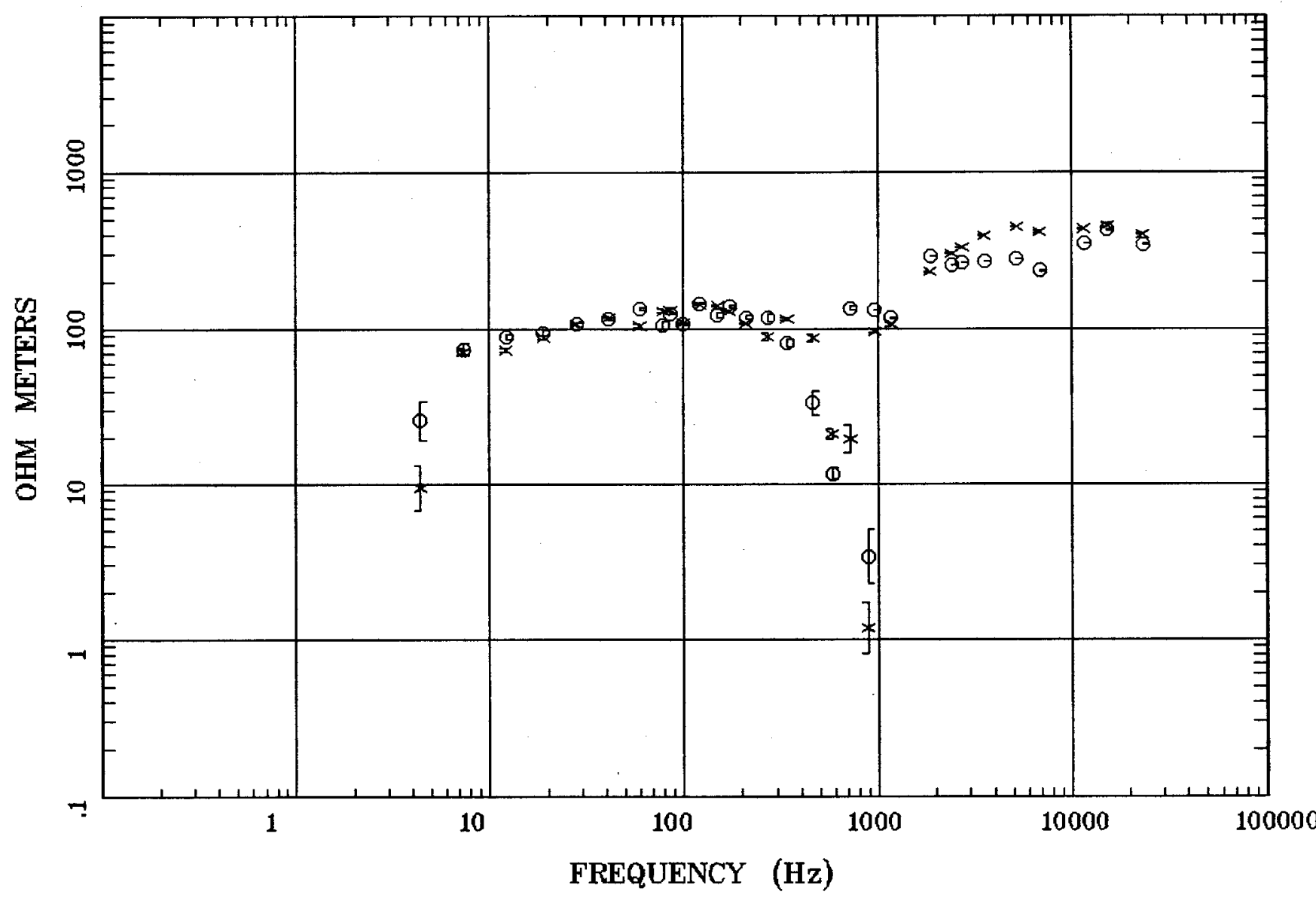

Client: Water Resources Remote: none

Acquired: 12:5 Nor 09, 2003 Survey Co:USGS
Rotation:

Filename: nts03.avg

Channels: Ch1 Ch2 Ch3 Ch4 Ch5 Ch3 Ch4 Plotted: 08:44 Dec 07, 2004

$<$ EMI - ElectroMagnetic Instruments > 


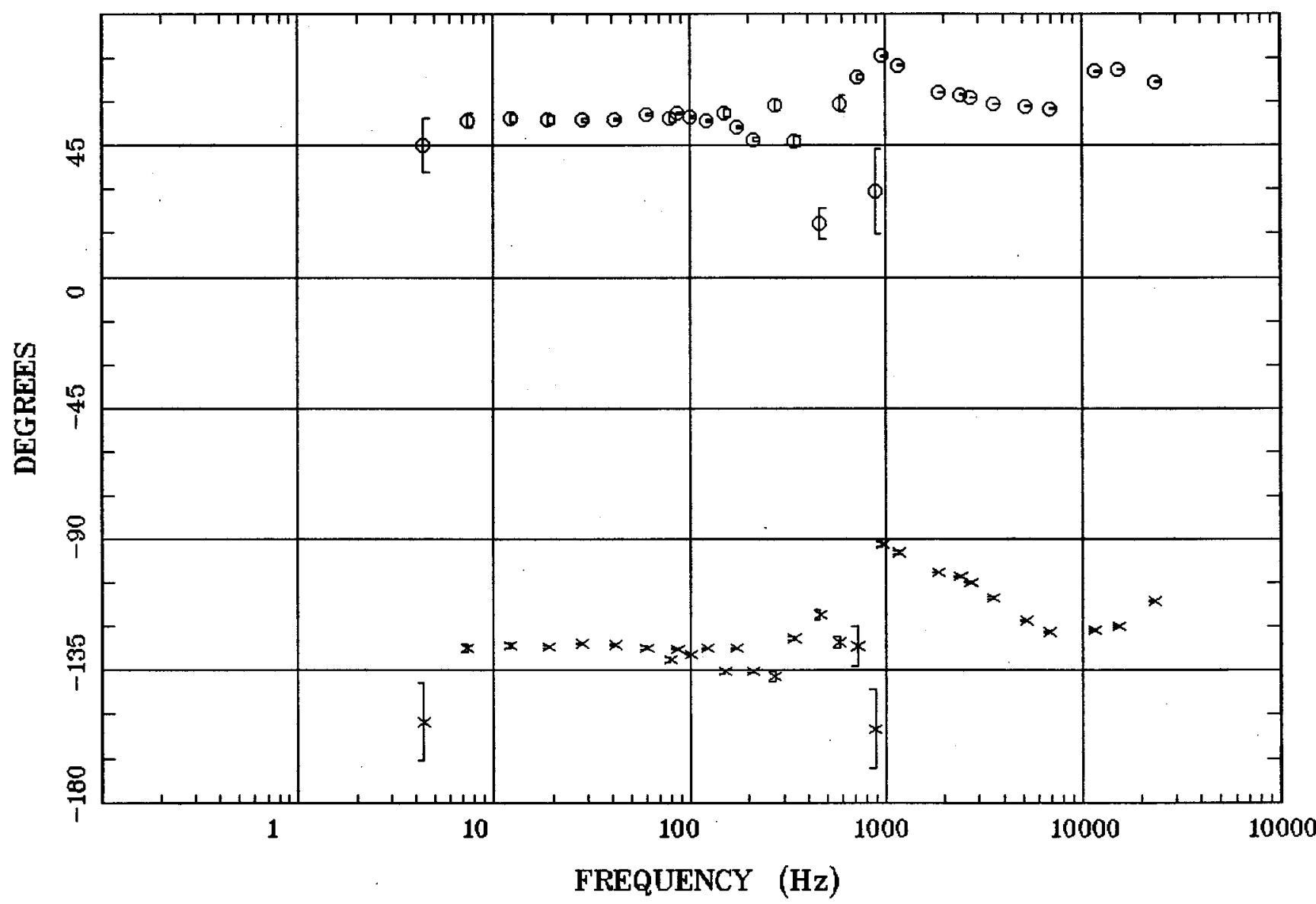

Client: Water Resources Remote: none Acquired: 12:5 Nov 09, 2003 Survey Co:USGS
Rotation:

Filename: nts03.avg

Channels: Ch1 Ch2 Ch3 Ch4 Ch5 Ch3 Ch4 Plotted: 08:44 Dec 07, 2004

< EMI - ElectroMagnetic Instruments > 


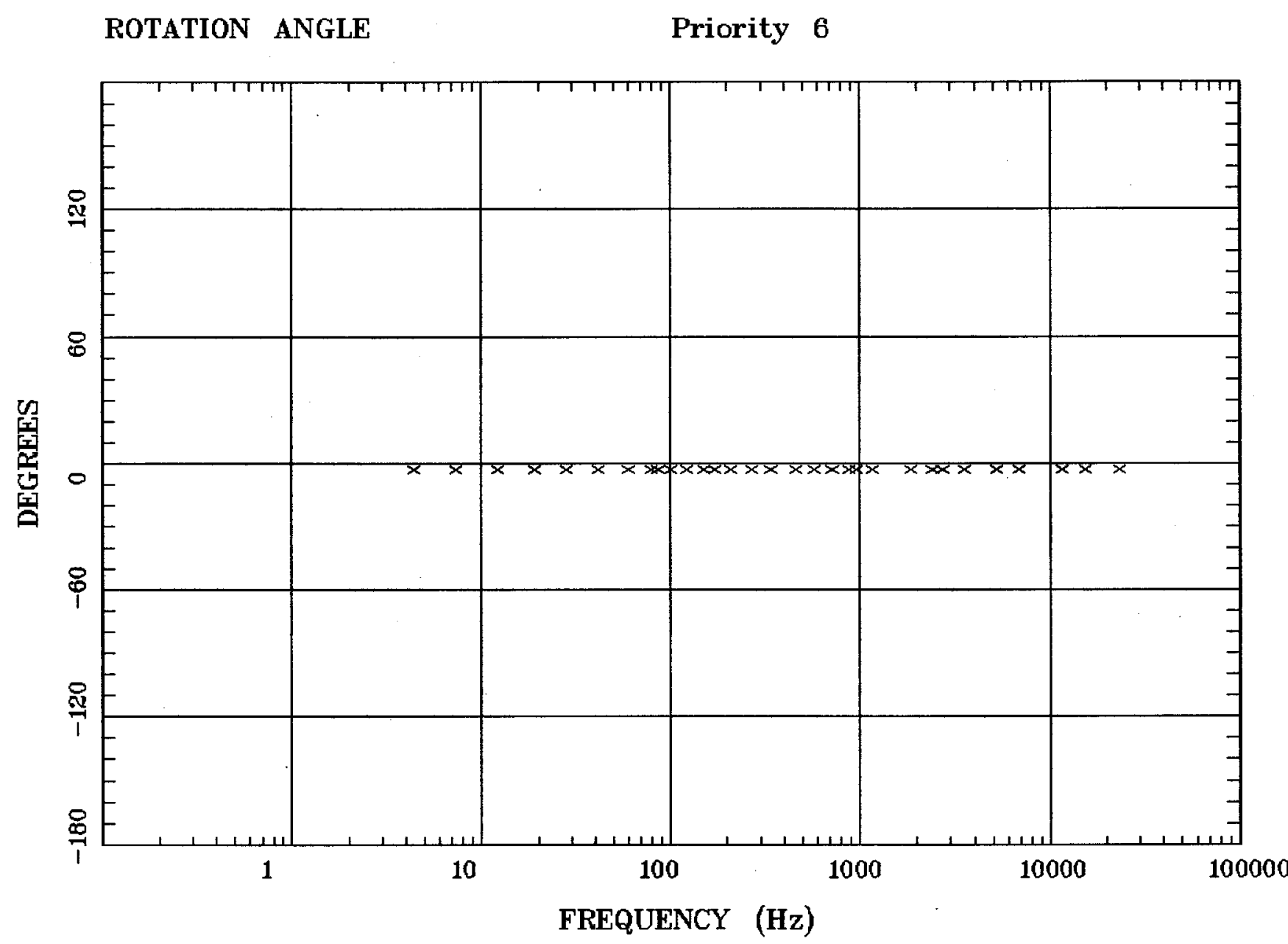

Client: Water Resources

Remote: none

Acquired: 12:5 Nov 09, 2003

Survey Co:USGS
Rotation:

Filename: nts03.avg

Channels: Ch1 Ch2 Ch3 Ch4 Ch5 Ch3 Ch4

Plotted: 08:44 Dec 07, 2004

< EMI - ElectroMagnetic Instruments 


\section{IMPEDANCE SKEW}

Priority 6

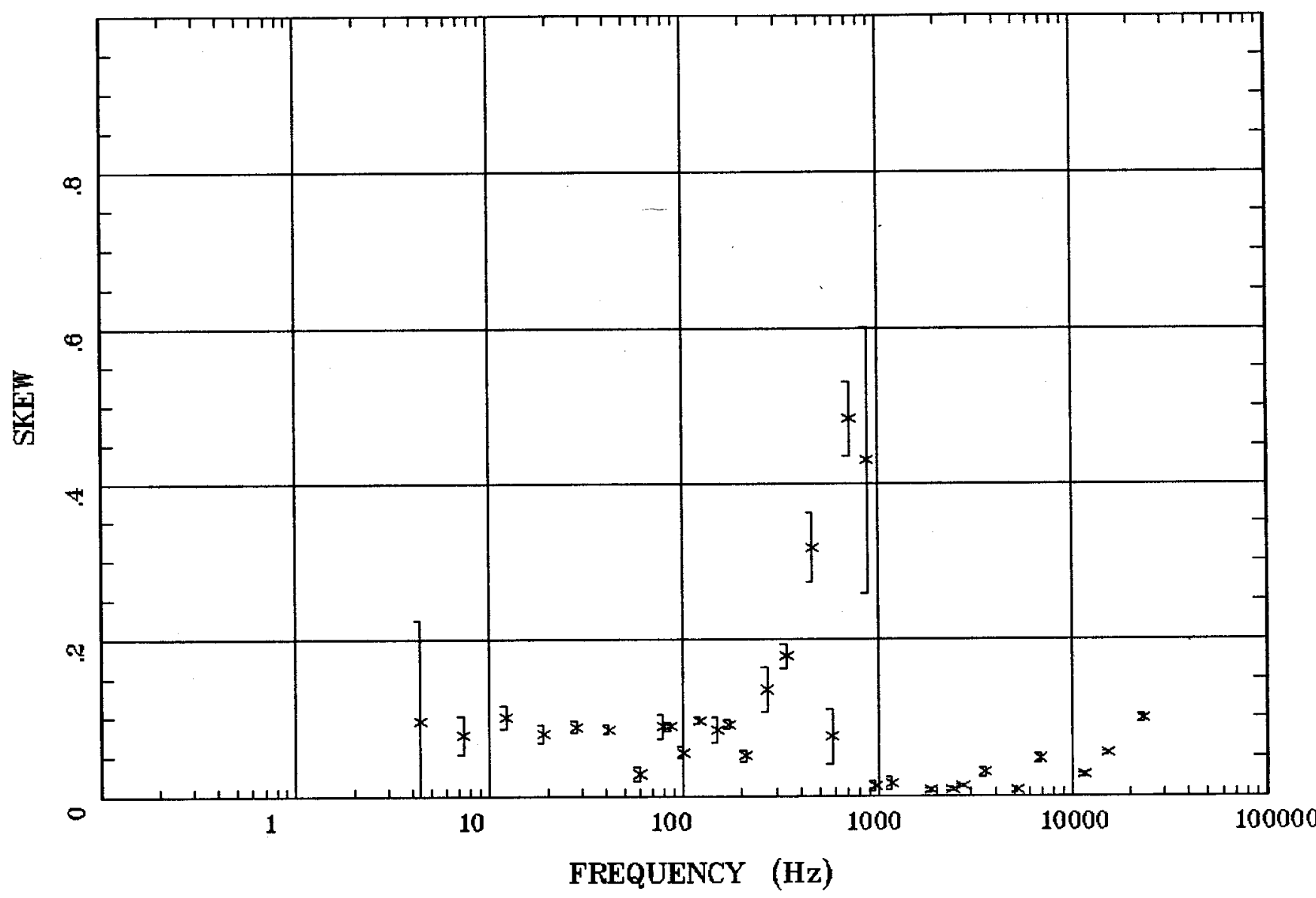

Client: Water Resources

Remote: none

Acquired: 12:5 Nov 09, 2003

Survey Co:USGS
Rotation:

Filename: nts03.avg

Channels: Ch1 Ch2 Ch3 Ch4 Ch5 Ch3 Ch4 Plotted: 08:44 Dec 07, 2004

< EMI - ElectroMagnetic Instruments > 


\section{E MULT Coh.}

Priority 6

Station 3

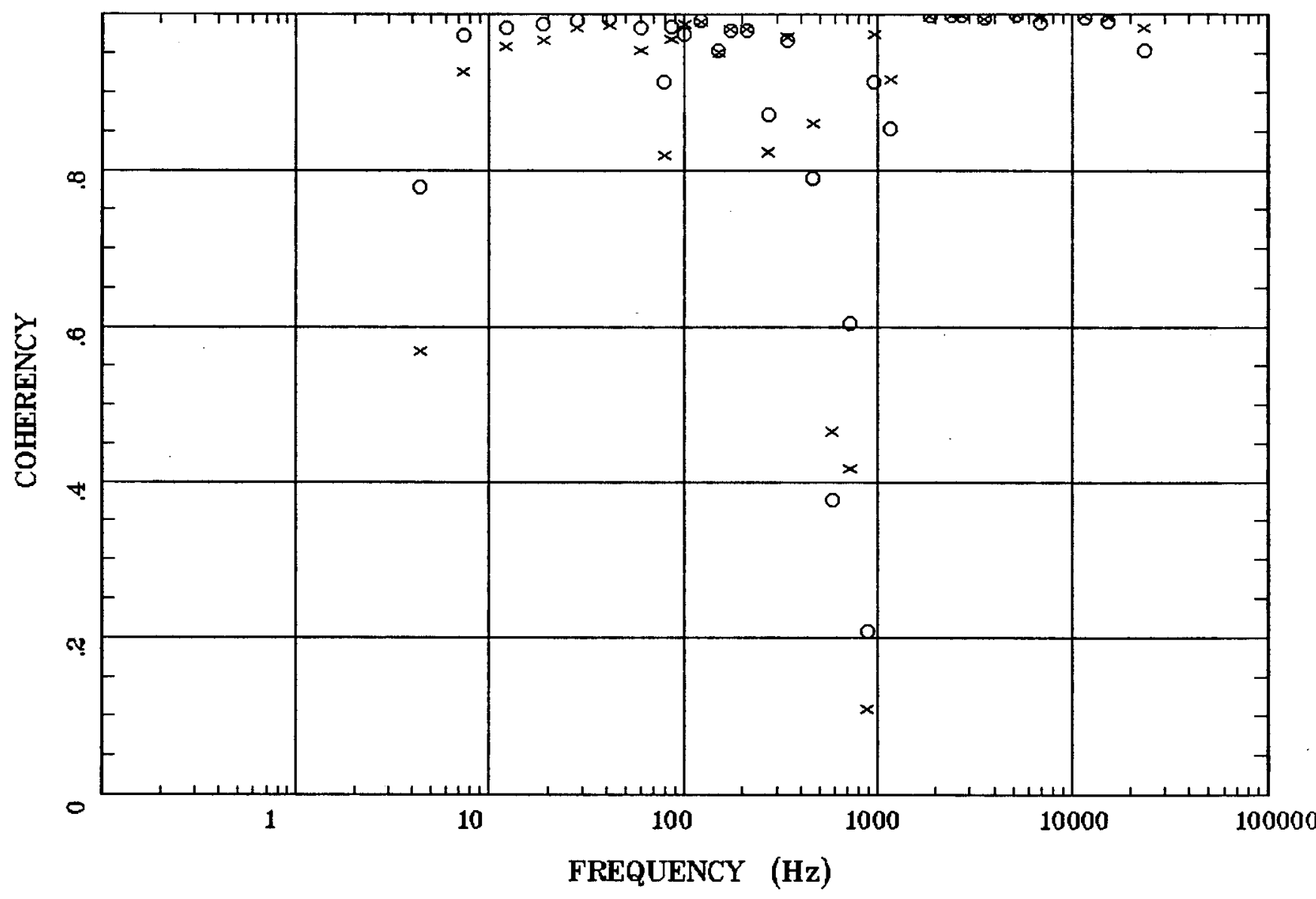

Client: Water Resources Remate: none Acquired: 12:5 Nov 09, 2003 Survey Co:USGS
Rotation:

Filename: nts03.avg

Channels: Ch1 Ch2 Ch3 Ch4 Ch5 Ch3 Ch4 Plotted: 08:44 Dec 07, 2004

< EMI - ElectroMagnetic Instruments 


\section{POLAR PLOTS}

\section{Priority 6}

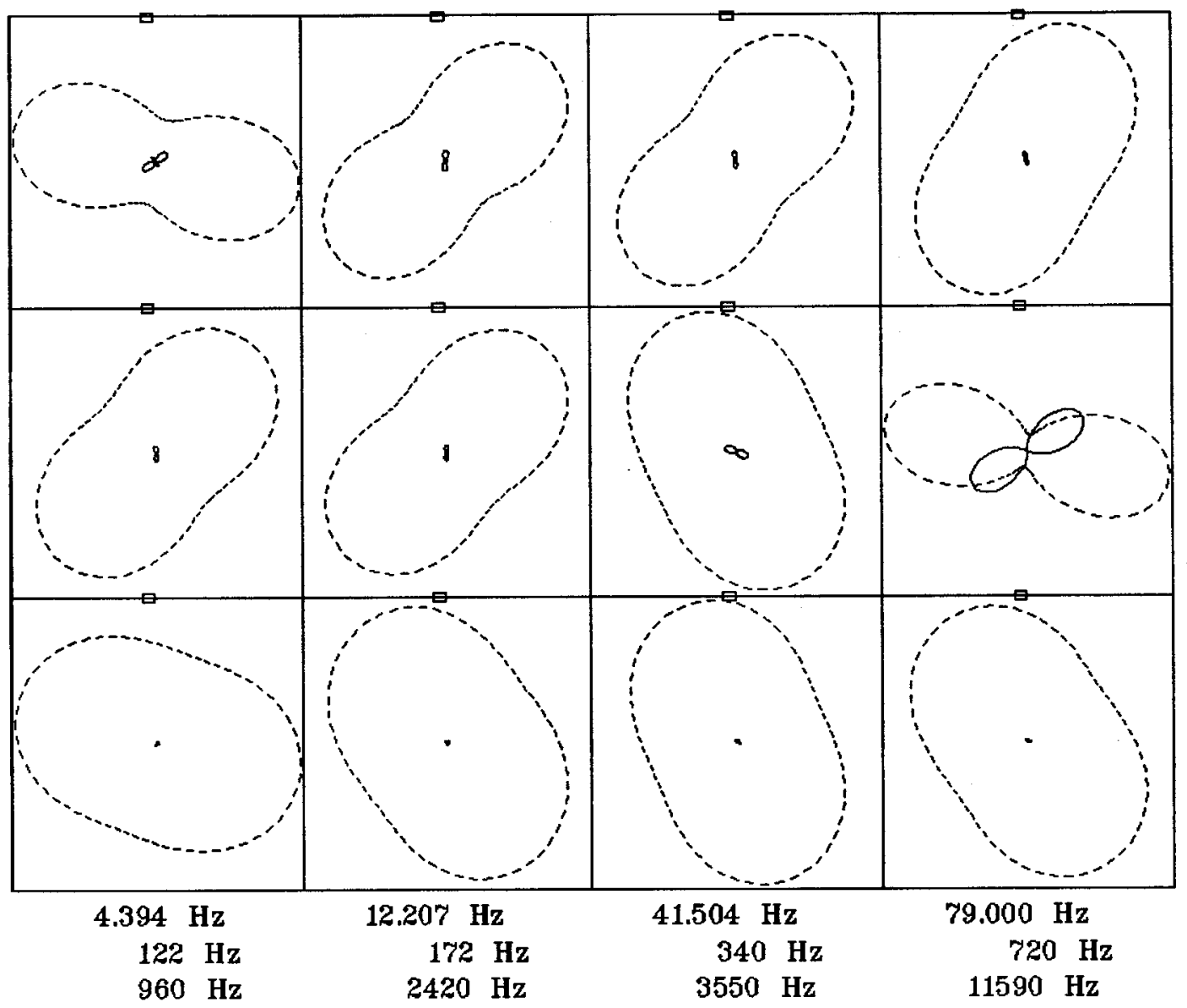

Rotation:

Client: Water Resources Remote: none

Acquired: 12:5 Nov 09, 2003 Survey Co:USGS
Filename: nts03.avg

Channels: Ch1 Ch2 Ch3 Ch4 Ch5 Ch3 Ch4 Plotted: 08:44 Dec 07, 2004

< EMI - ElectroMagnetic Instruments 


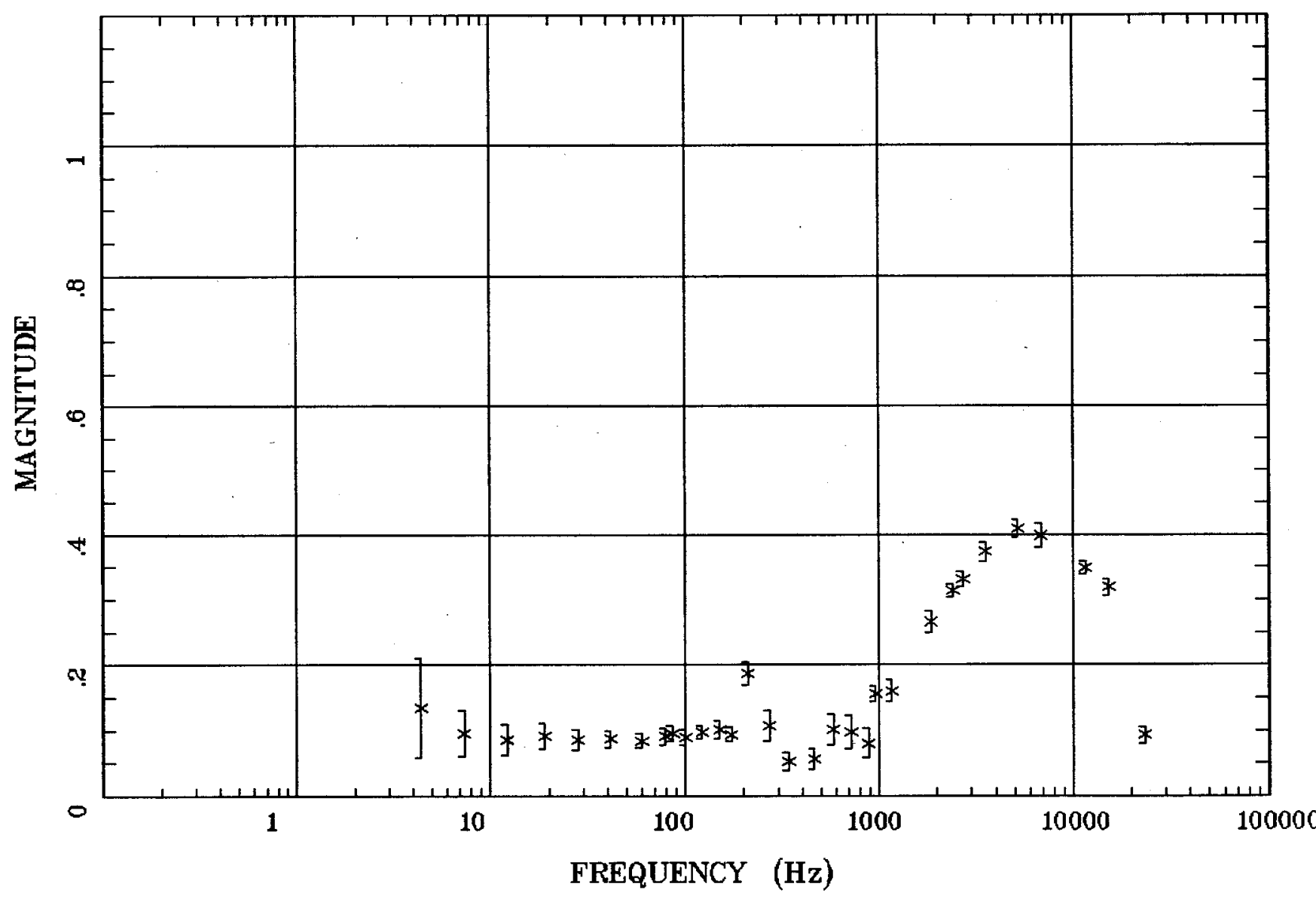

Client: Water Resources Remote: none Acquired: 12:5 Nov 09, 2003 Survey Co:USGS

\section{Rotation:}

Filename: nts03.avg

Channels: Ch1 Ch2 Ch3 ch4 Ch5 Ch3 Ch4 Plotted: 08:44 Dec 07, 2004

< EMI - ElectroMagnetic Instruments > 


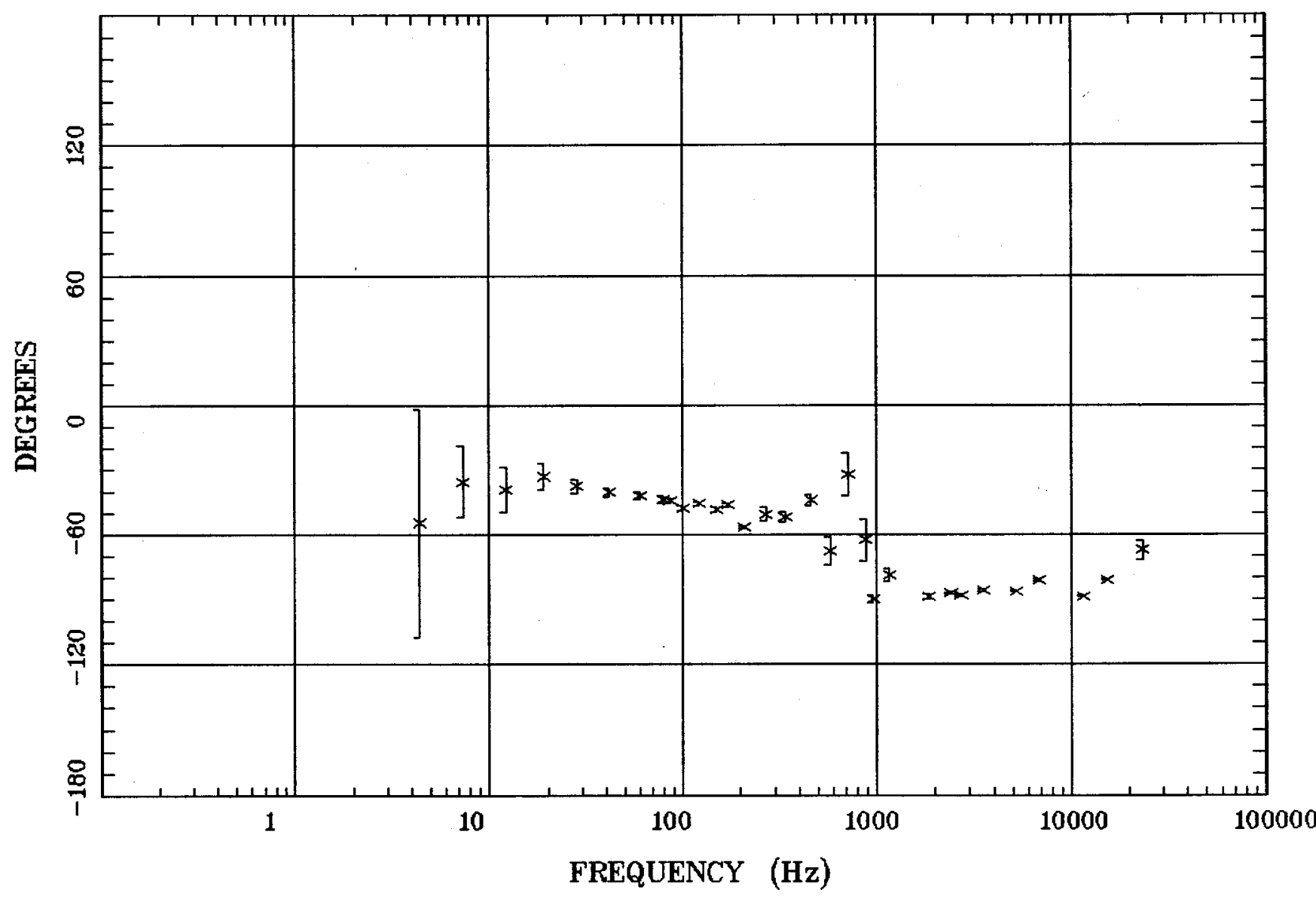

Client: Water Resources Remote: none

Acquired: 12:5 Nov 09, 2003 Survey Co:USGS
Rotation:

Filename: nts03.avg

Channels: Ch1 Ch2 Ch3 Ch4 Ch5 Ch3 Ch4

Plotted: 08:44 Dec 07, 2004

< EMI - ElectroMagnetic Instruments > 
HzHx.x Coh HzHy.o

Priority 6

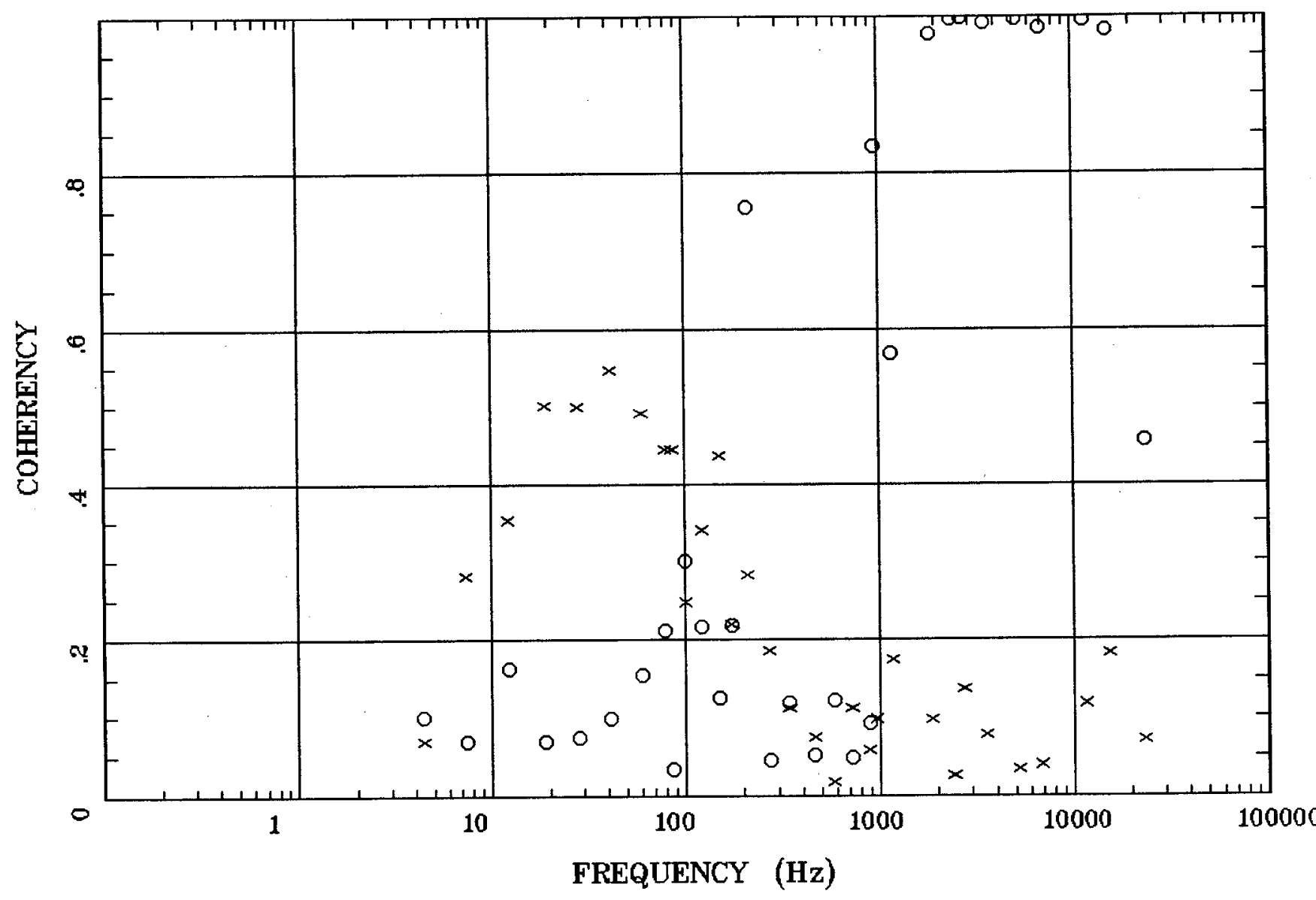

Client: Water Resources Remote: none

Acquired: 12:5 Nov 09, 2003 Survey Co:USGS
Rotation:

Filename: nts03.avg

Channels: Ch1 Ch2 Ch3 Ch4 Ch5 Ch3 Ch4

Platted: 08:44 Dec 0r, 2004

$<$ EMI - ElectroMagnetic Instruments > 
APPARENT RESISTIVITY

Priority 6

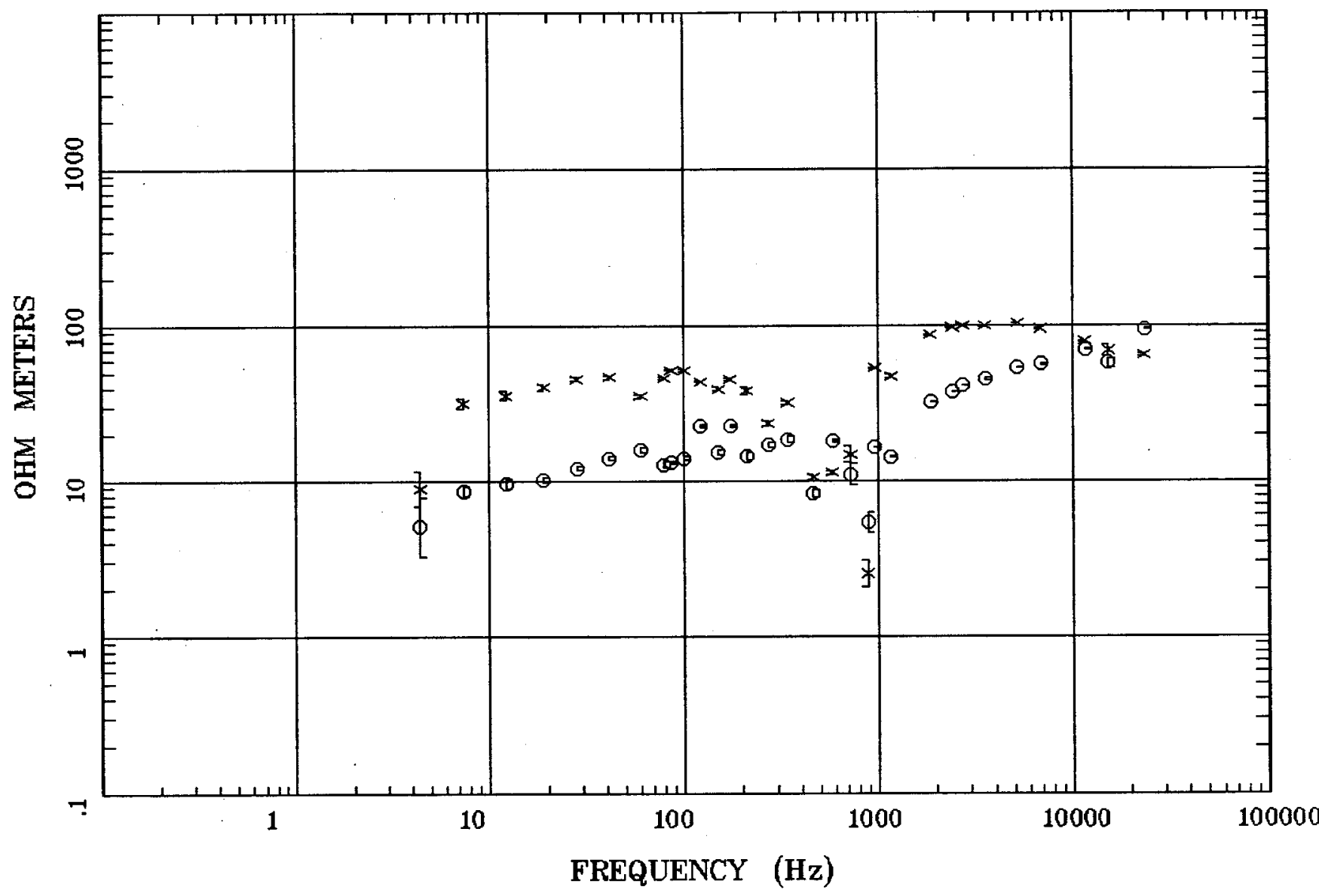

Client: Water Resources Remote: none Acquired: 15:3 Nov 09, 2003 Survey Co:USGS
Rotation:

Filename: nts04.avg

Channels: Ch1 Ch2 Ch3 Ch4 Ch5 Ch3 Ch4 Plotted: 08:44 Dec 07, 2004

< EMI - ElectroMagnetic Instruments 


\section{IMPEDANCE PHASE}

\section{Priority 6}

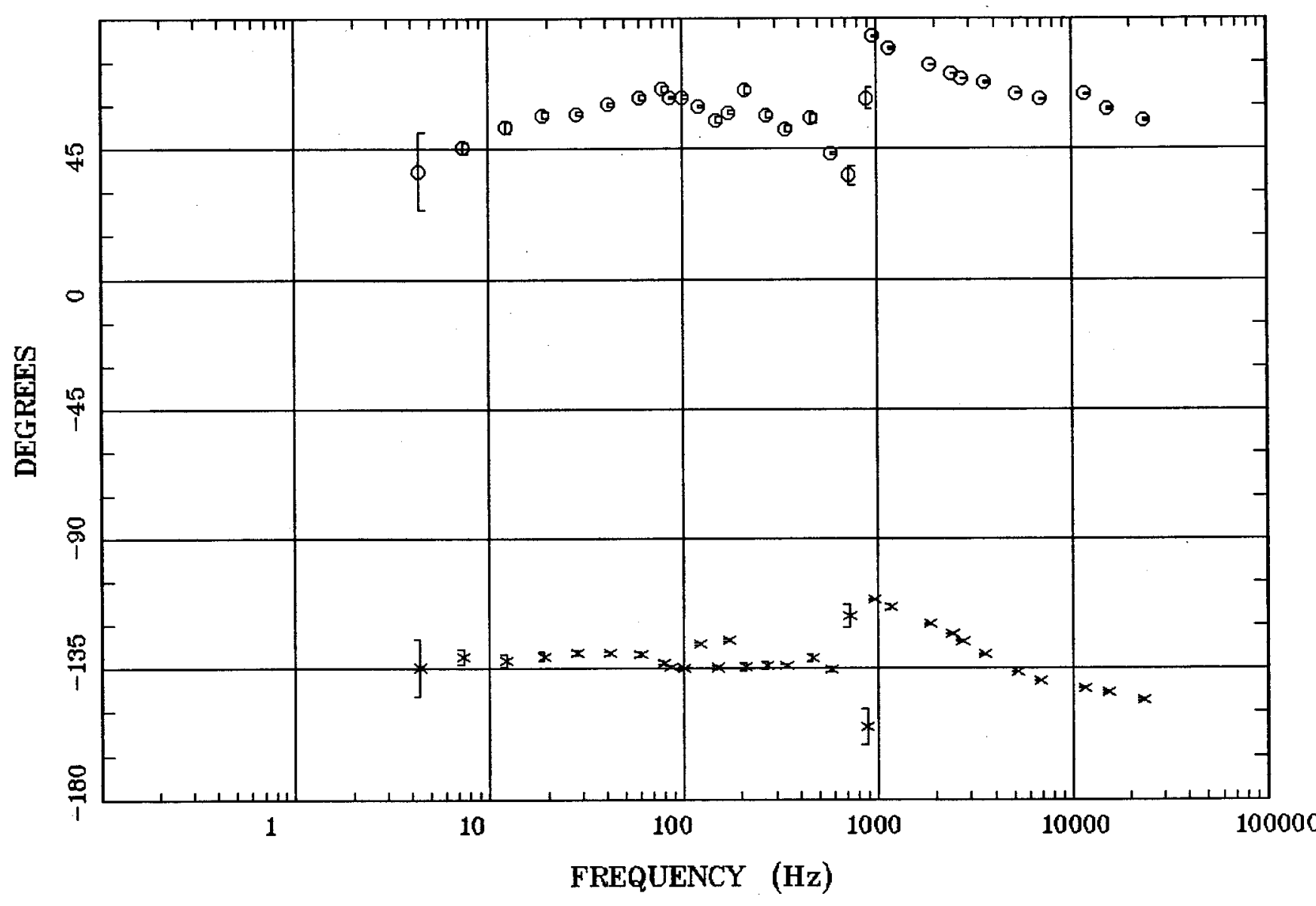

Client: Water Resources Remote: none Acquired: 15:3 Nav 09, 2003 Survey Co:USGS
Rotation:

Filename: nts04.avg

Channels: Ch1 Ch2 Ch3 Ch4 Ch5 Ch3 Ch4 Plotted: 08:44 Dec 07, 2004

< EMI - ElectroMagnetic Instruments > 


\section{ROTATION ANGLE}

Priority 6

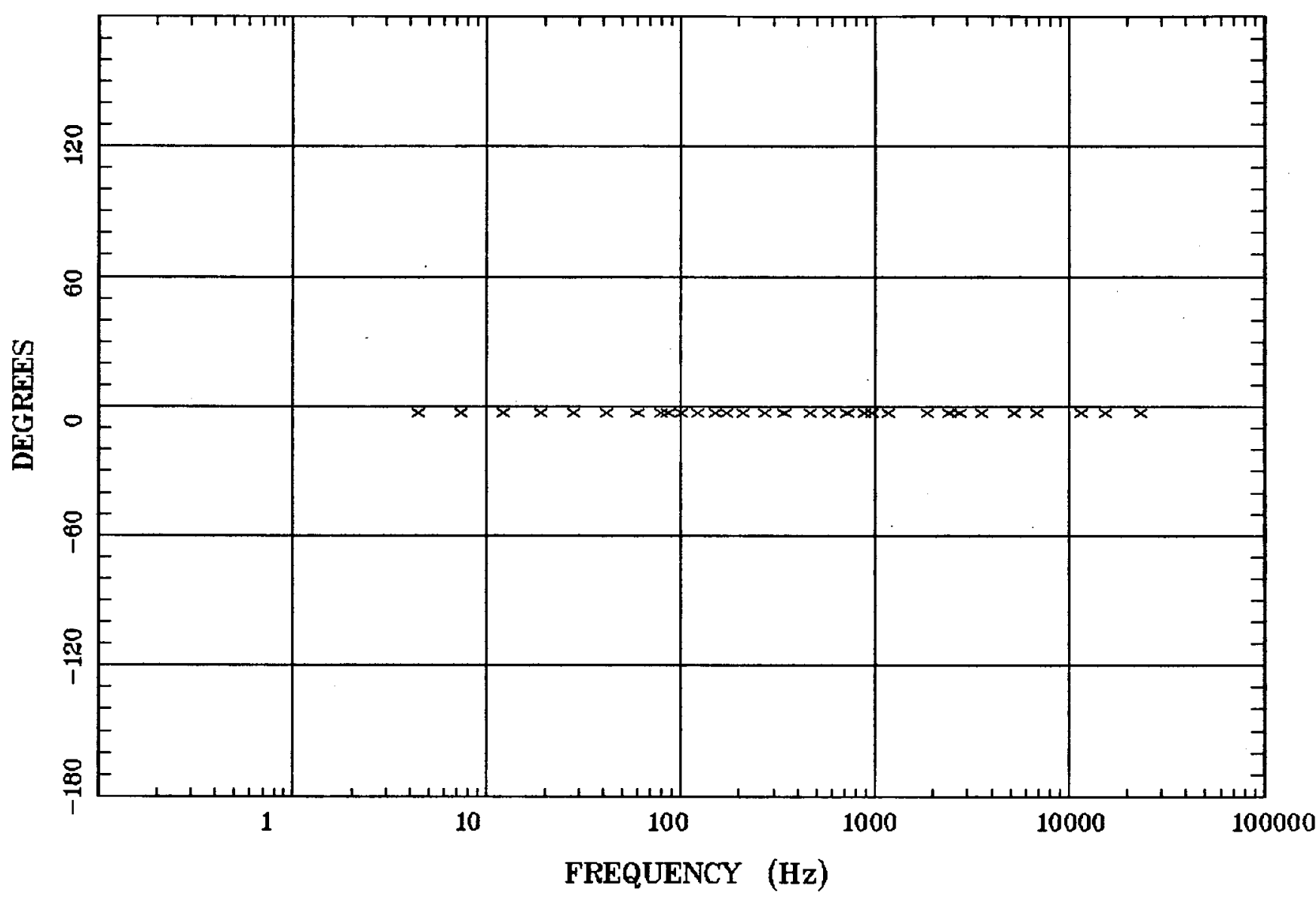

Client: Water Resources

Remote: none

Acquired: 15:3 Nov 09, 2003

Survey Co:USGS
Rotation:

Filename: nts04.avg

Channels: Ch1 Ch2 Ch3 Ch4 Ch5 Ch3 Ch4

Plotted: 08:45 Dec 07. 2004

< EMI - ElectroMagnetic Instruments > 


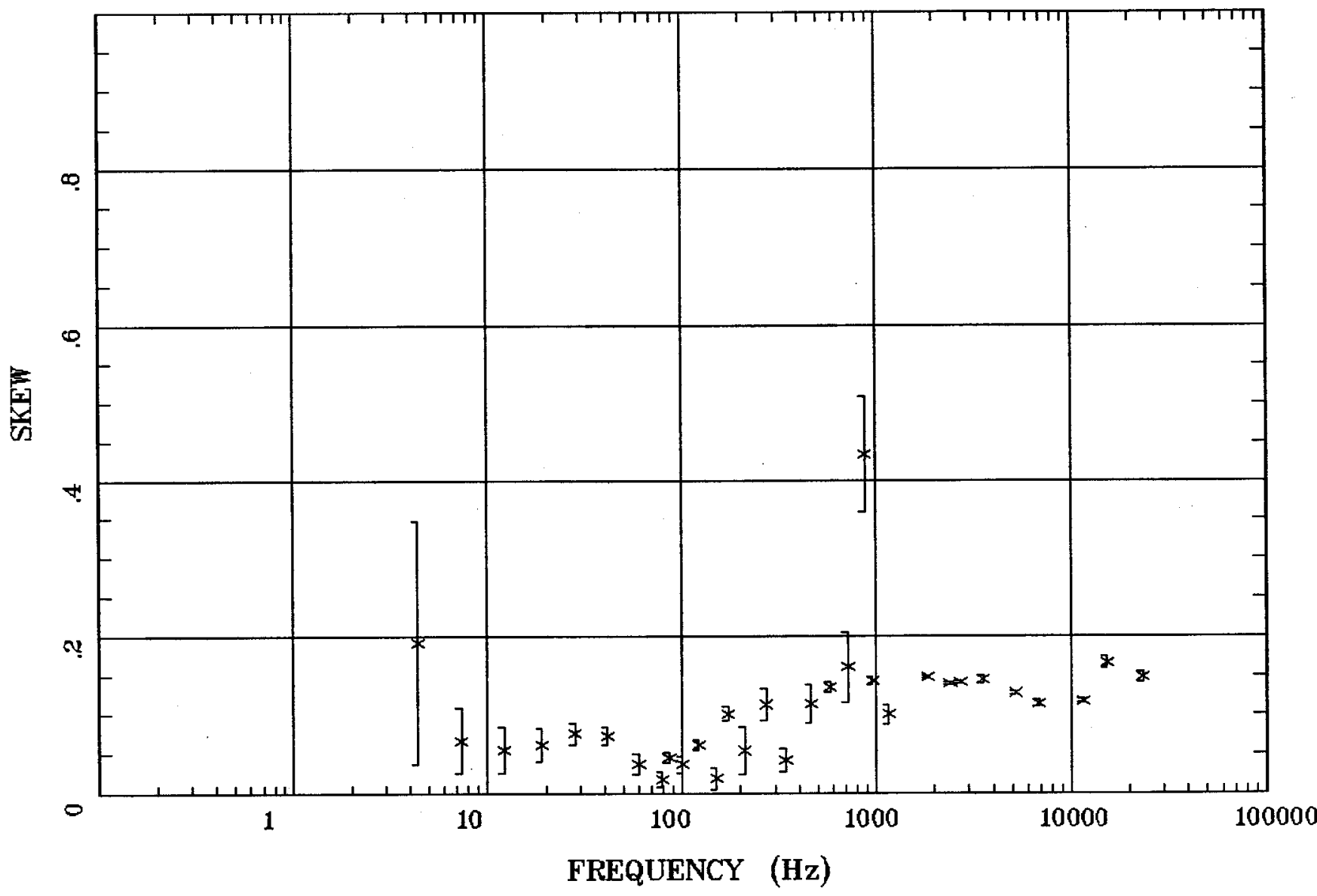

Client: Water Resources

Remote: none

Acquired: $\quad 15: 3$ Nov 09, 2003

Survey ca:UsGS
Rotation:

Filename: nts04.avg

Channels: Ch1 Ch2 Ch3 Ch4 Ch5 Ch3 Ch4

Plotted: 08:45 Dec 07, 2004

$<$ EMI - ElectroMagnetic Instruments 


\section{E MULT Coh. Priority 6}

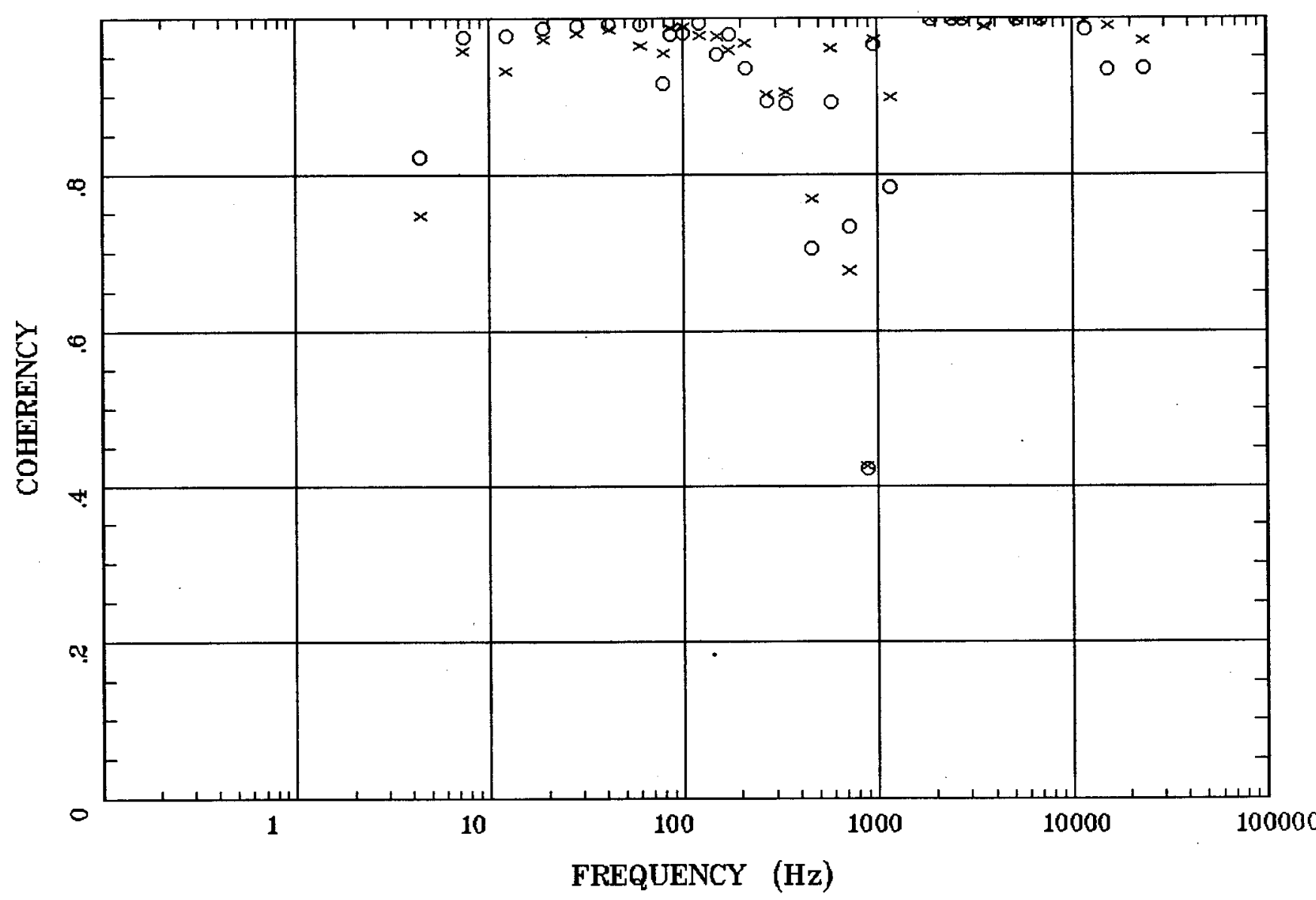

Client: Water Resources

Remote: none

Acquired: 15:3 Nov 09, 2003 Survey Co:USGS
Rotation:

Filename: nts04.avg

Channels: Ch1 Ch2 Ch3 Ch4 Ch5 Ch3 Ch4 Plotted: 08:45 Dec 07, 2004

< EMI - ElectroMagnetic Instruments > 
Station 4

POLAR PLOTS

Priority 6

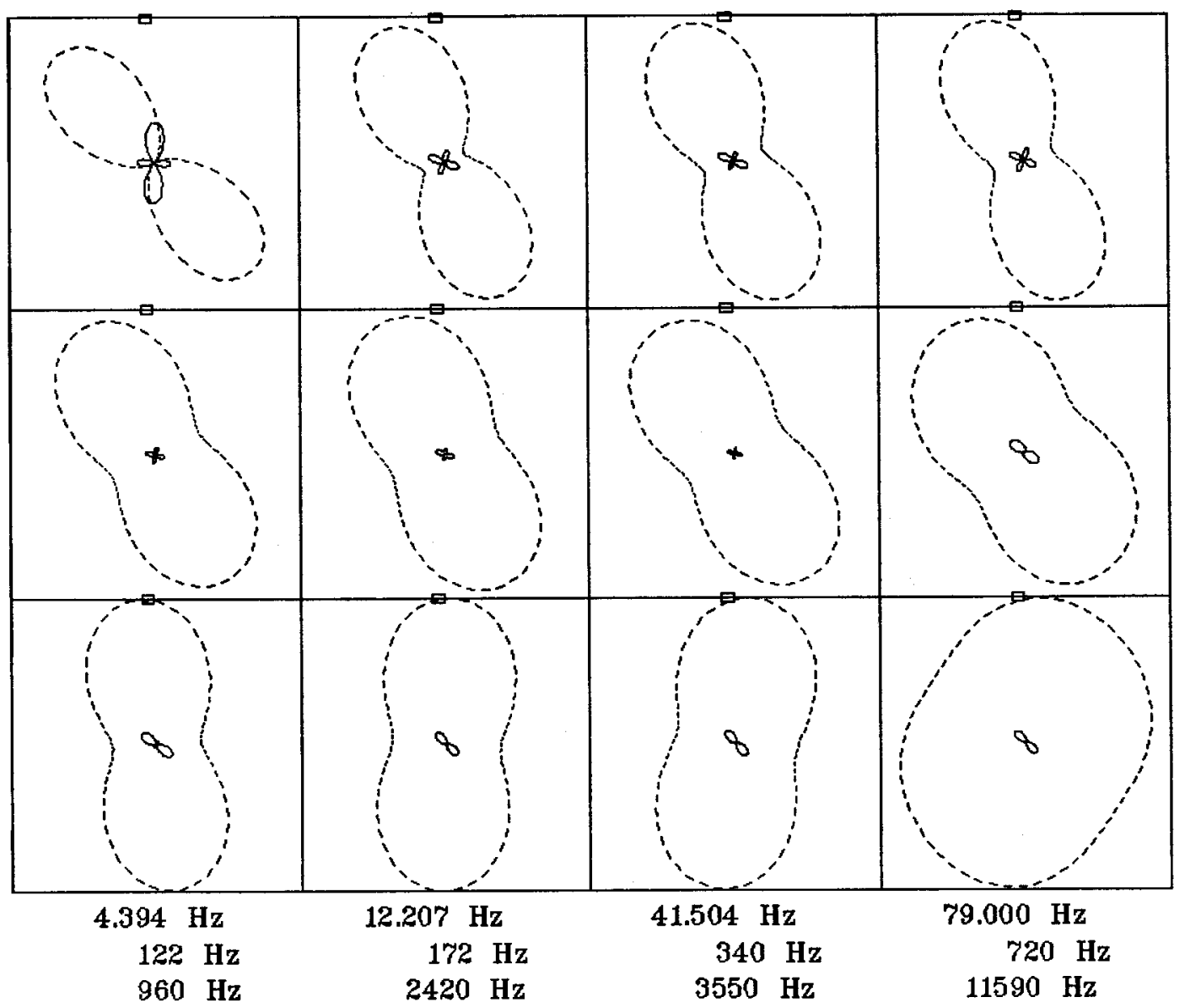

Client: Water Resources

Rotation:

Filename: nts04.avg

Remote: none

Acquired: 15:3 Nov 09, 2003

Channels: Ch1 Ch2 Ch3 Ch4 Ch5 Ch3 Ch4

Plotted: 08:45 Dec 07, 2004

Survey Co:USGS

< EMI - ElectroMagnetic Instruments > 


\section{TIPPER MAGNITUDE}

Priority 6

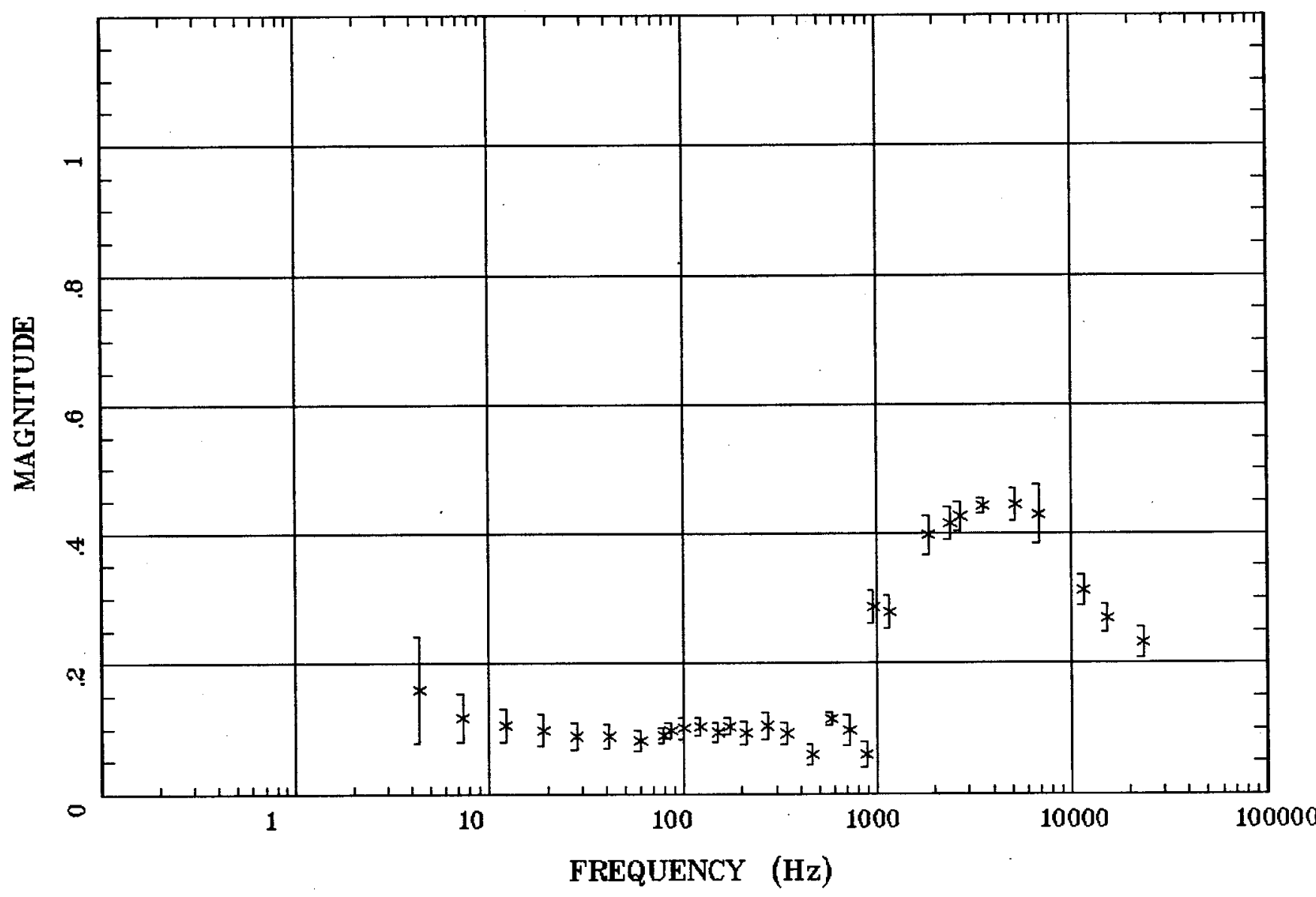

Client: Water Resources

Remote: none

Acquired: 15:3 Nov 09, 2003

Survey Co:USGS

\section{Rotation:}

Filename: nts04.avg

Channels: Ch1 Ch2 Ch3 Ch4 Ch5 Ch3 Ch4 Plotted: 08:45 Dec 07, 2004

< EMI - ElectroMagnetic Instruments > 


\section{TIPPER STRIKE}

Priority 6

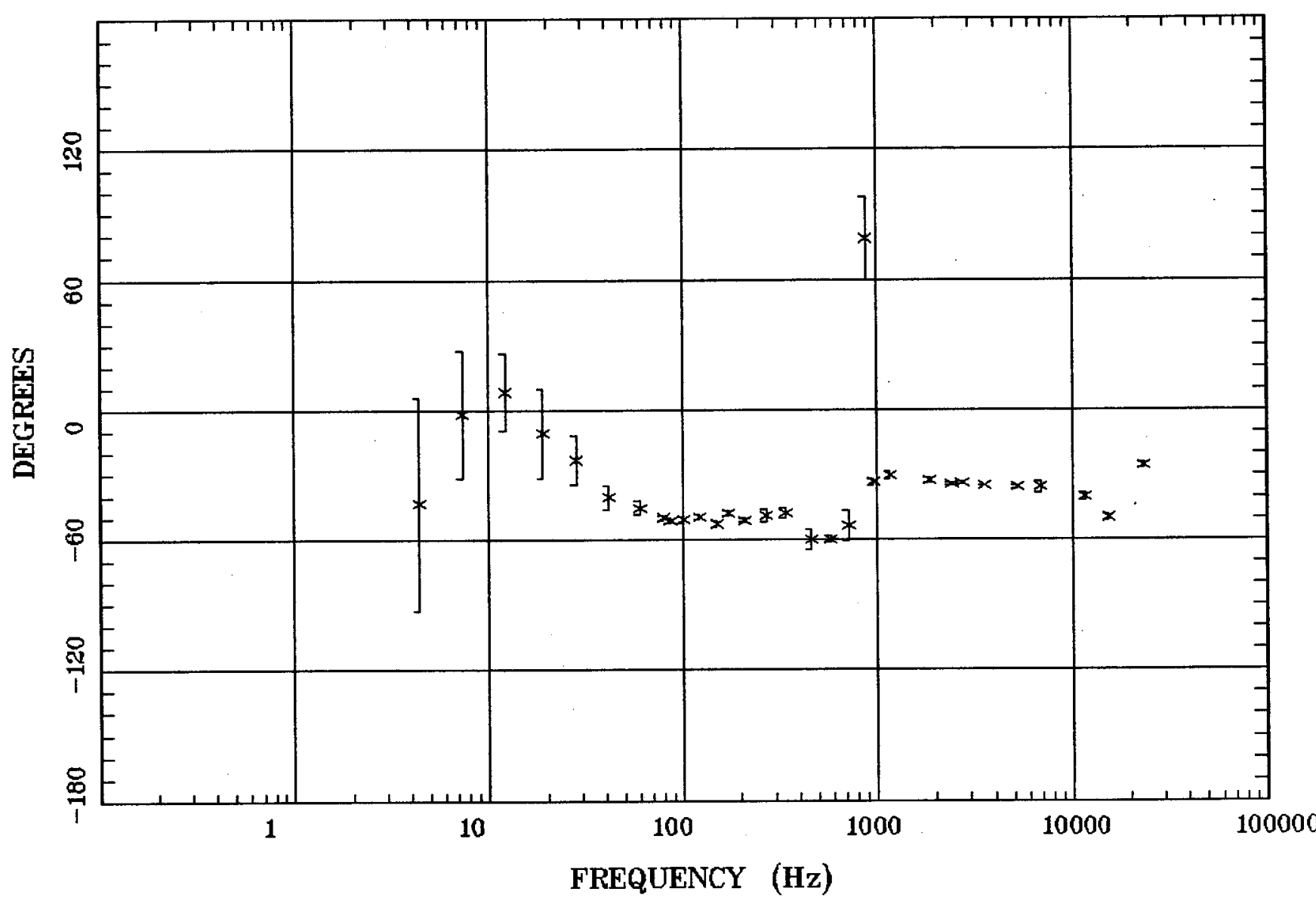

Client: Water Resources

Remote: none

Acquired: 15:3 Nov 09, 2003

Survey Co:USGS
Rotation:

Filename: nts04.avg

Channels: Ch1 Ch2 Ch3 Ch4 Ch5 Ch3 Ch4

Plotted: 08:45 Dec 07, 2004

< EMI - ElectroMagnetic Instruments > 
HzHx.x Coh HzHy.o

Priority 6

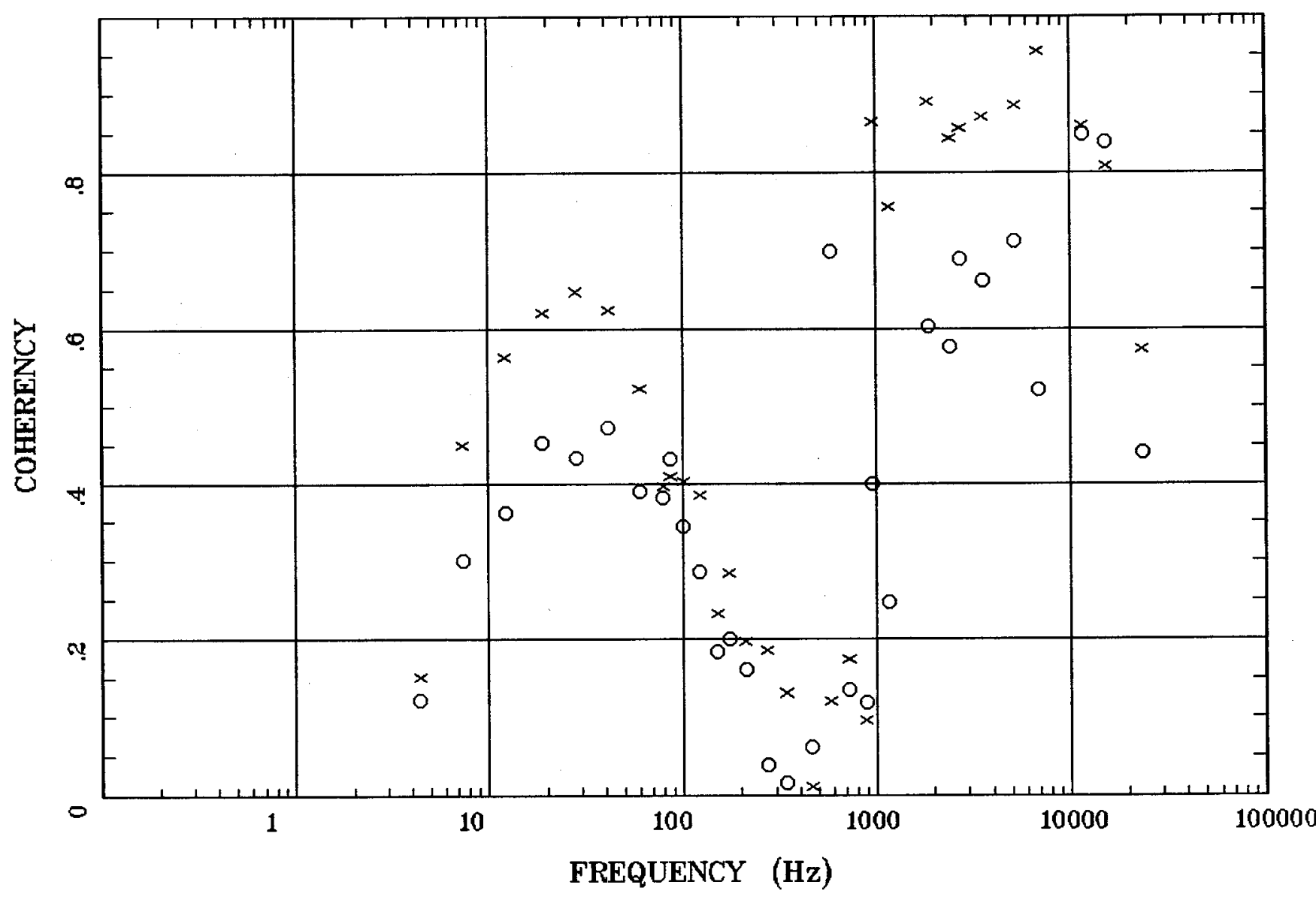

Client: Water Resources

Remote: none

Acquired: 15:3 Nov 09, 2003

Survey Co:USGS
Rotation:

Filename: nts04.avg

Channels: Ch1 Ch2 Ch3 Ch4 Ch5 Ch3 Ch4

Plotted: 08:45 Dec 07, 2004

< EMI - ElectroMagnetic Instruments > 


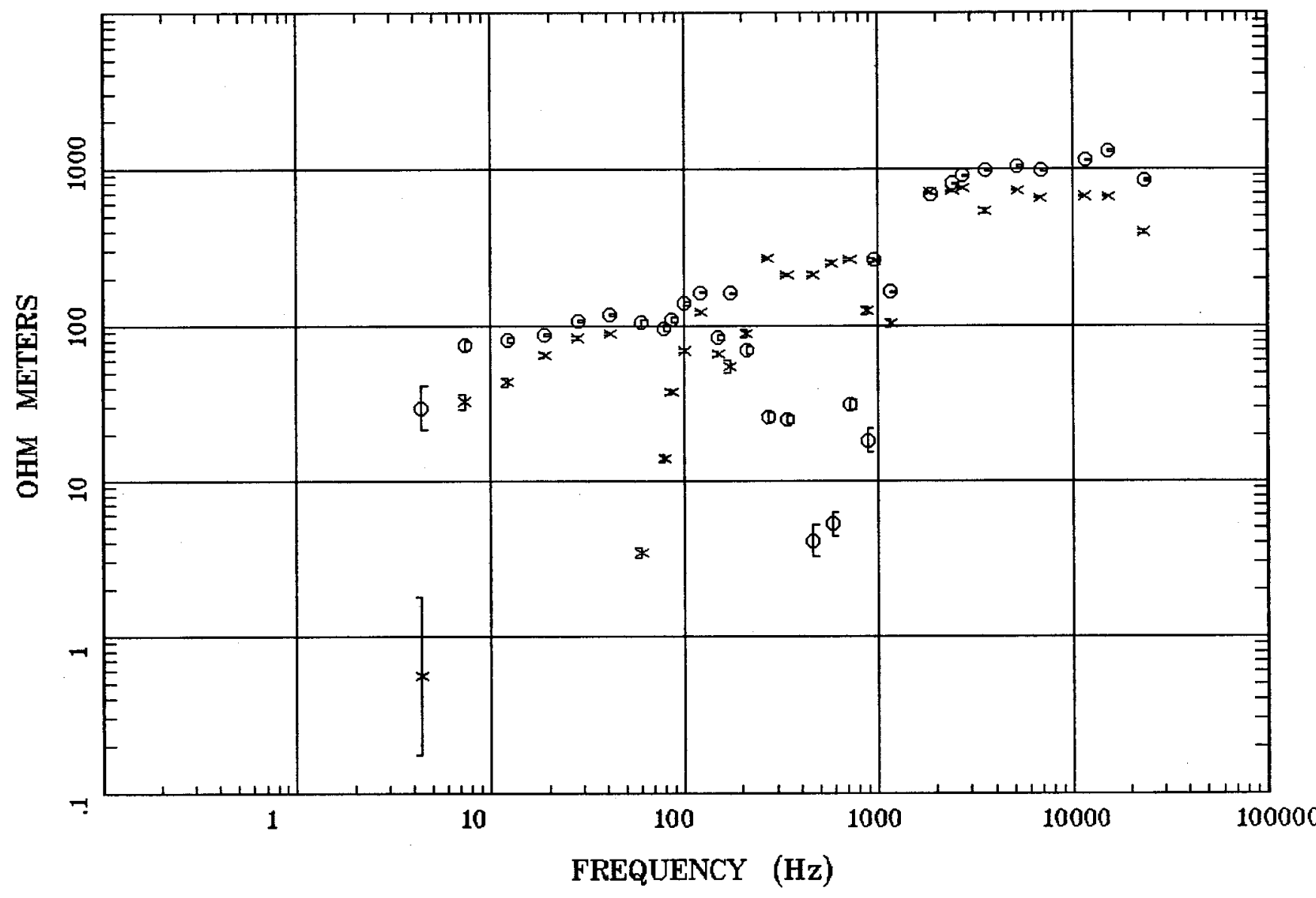

Client: Rater Resources Remote: none

Acquired: 11:0 Dec 05, 2003

Survey Co:USGS
Rotation:

Filename: nts5.avg

Channels: Ch1 Ch2 Ch3 Ch4 Ch5 Ch3 Ch4 Plotted: 08:45 Dec 07, 2004

< EMI - ElectroMagnetic Instruments > 


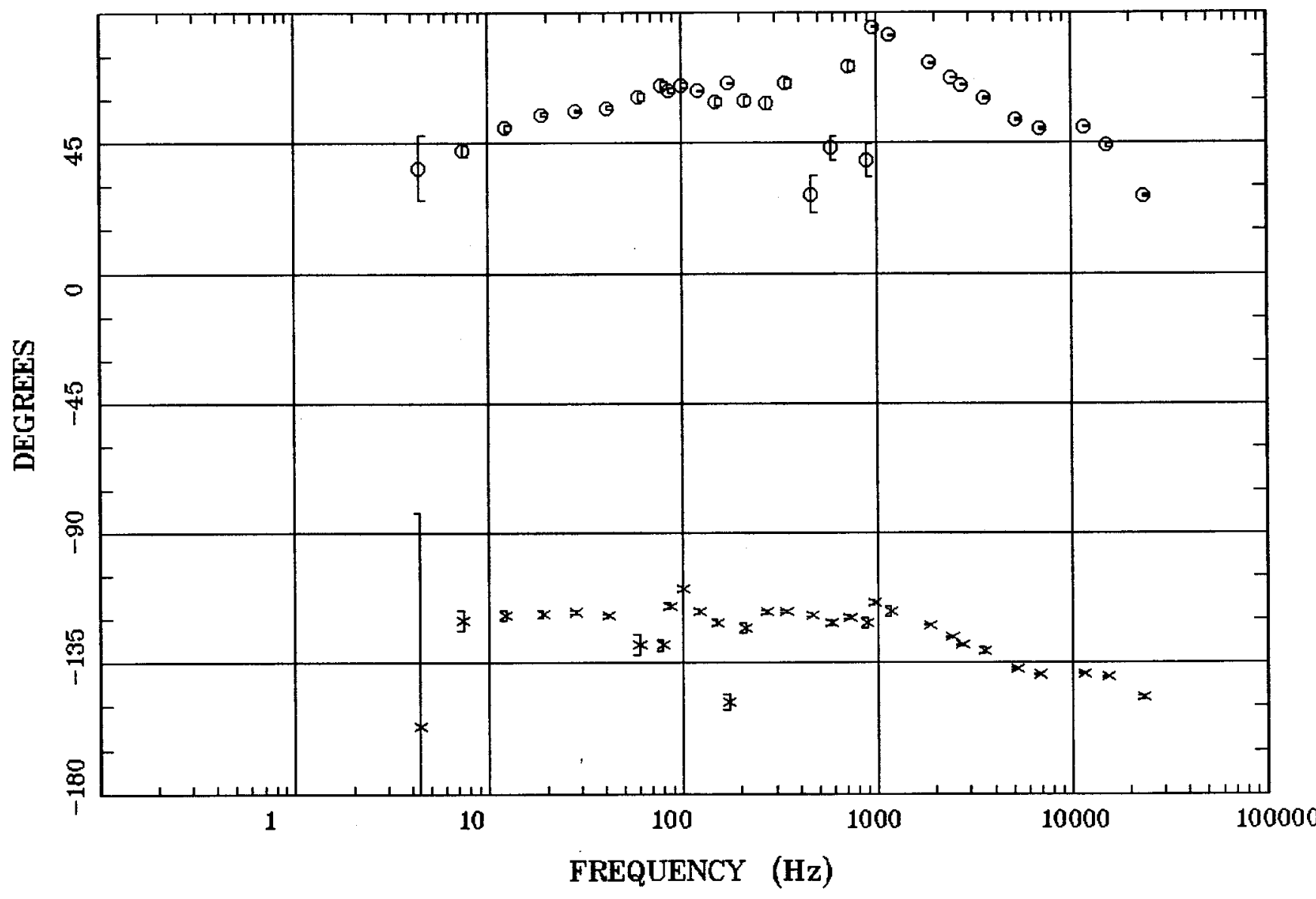

Client: Water Resources Remote: none Acquired: 11:0 Dec 05, 2003 Survey Co:USGS
Rotation:

Filename: nts5.avg

Channels: Ch1 Ch2 Ch3 Ch4 Ch5 Ch3 Ch4 Plotted: 08:45 Dec 07, 2004

< EMI - ElectroMagnetic Instruments > 


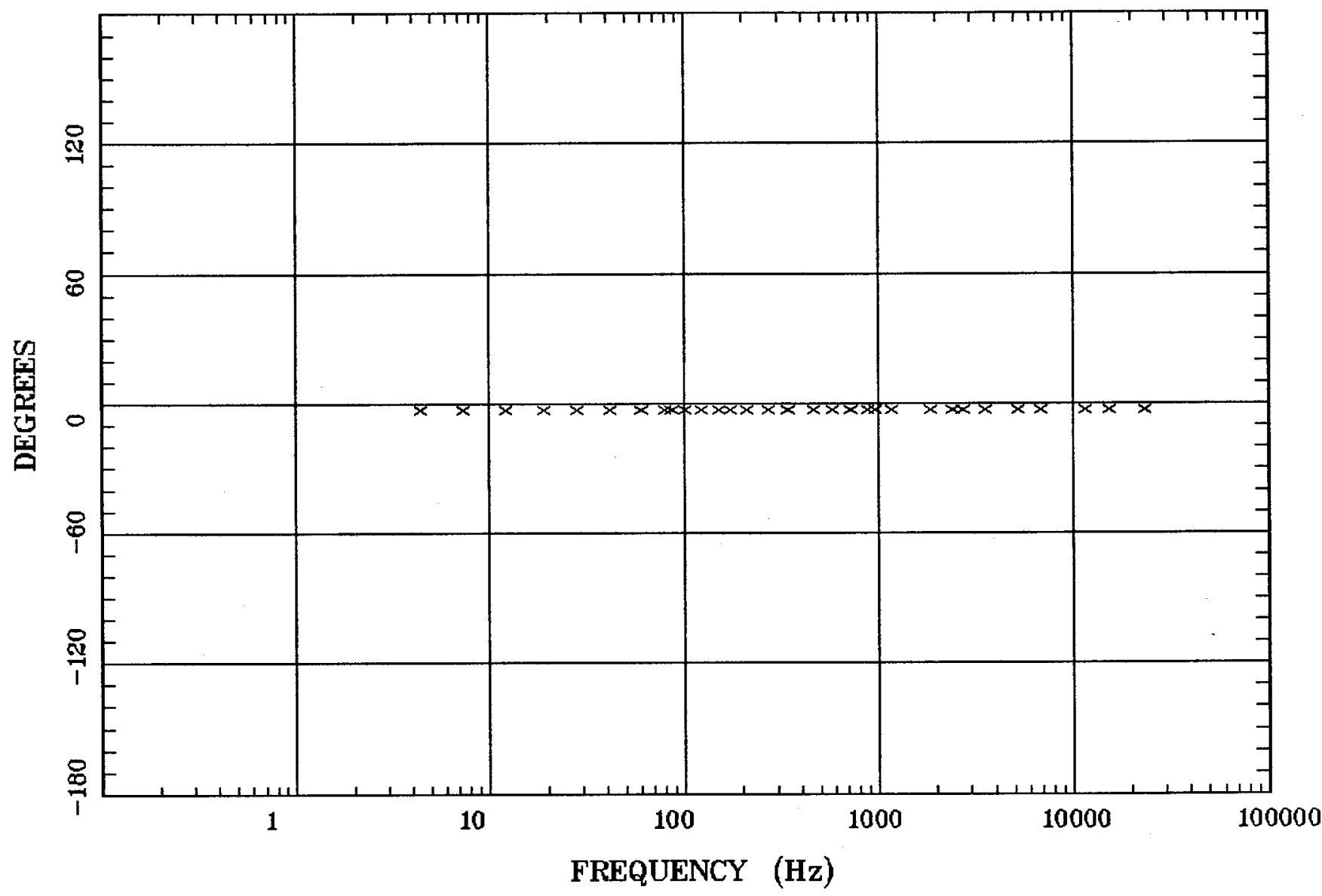

Client: Water Resources Remote: none Acquired: 11:0 Dec 05, 2003 Survey Co:USGS
Rotation:

Filename: nts5.avg

Channels: Ch1 Ch2 Ch3 Ch4 Ch5 Ch3 Ch4 Plotted: 08:45 Dec 07, 2004

< EMI - ElectroMagnetic Instruments > 


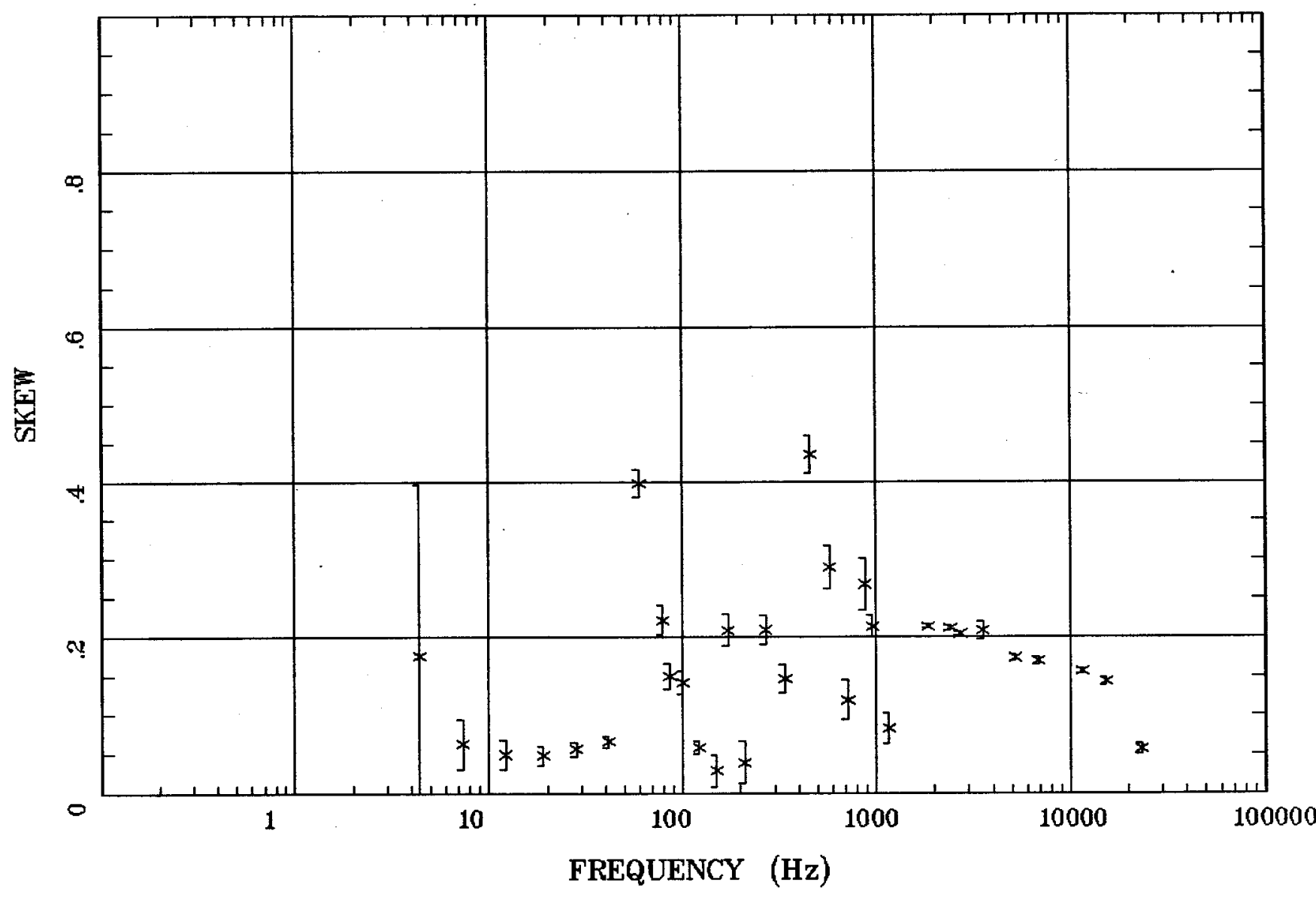

Client: Water Resources Remote: none Acquired: 11:0 Dec 05, 2003 Survey Co:USGS
Rotation:

Filename: nts5.avg

Channels: Ch1 Ch2 Ch3 ch4 Ch5 Ch3 Ch4 Plotted: 08:45 Dec 07, 2004

< EMI - ElectroMagnetic Instruments > 


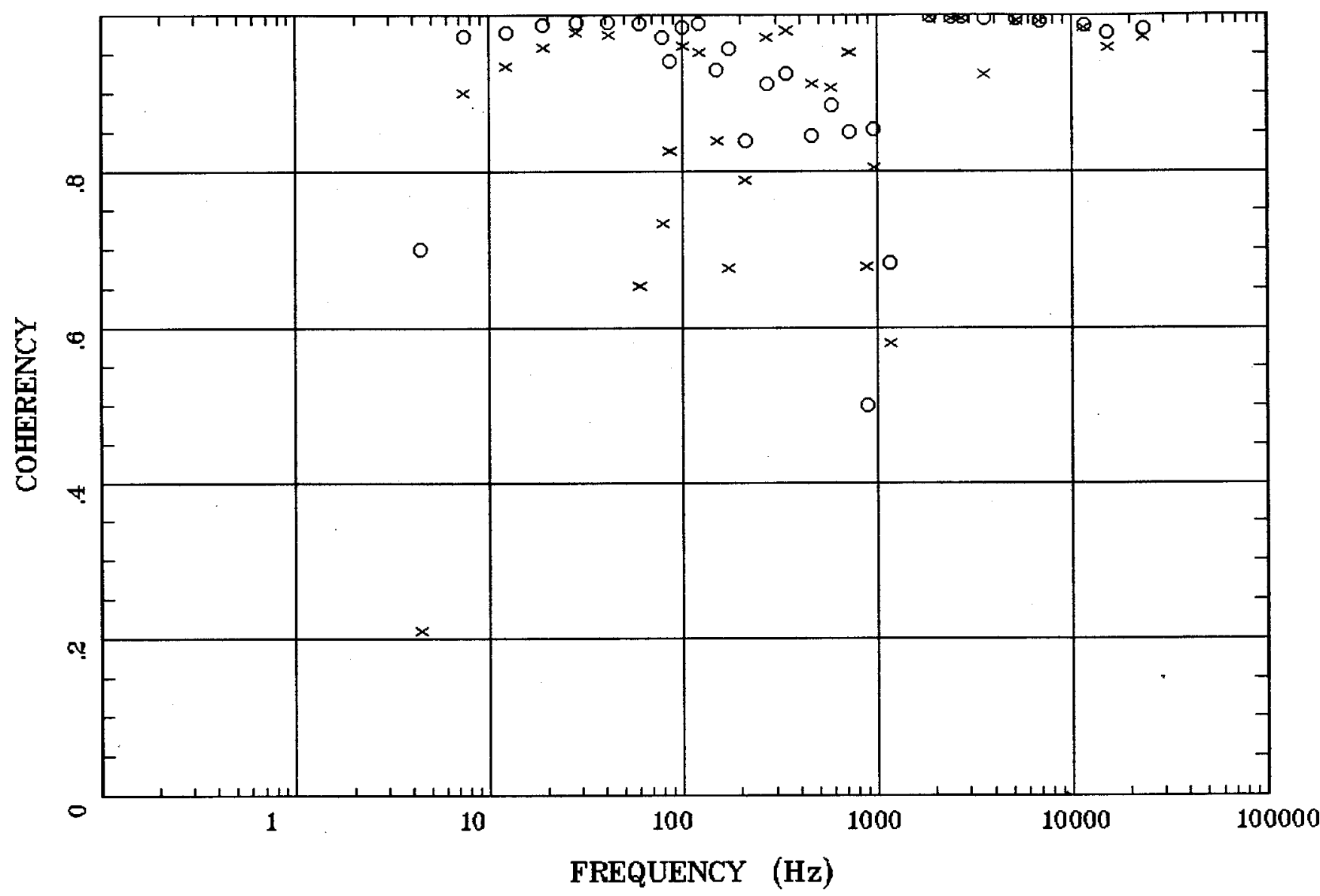

Client: Water Resources

Remote: none

Acquired: 11:0 Dec 05, 2003

Survey Co:USGS
Rotation:

Filename: nts5.avg

Channels: Ch1 Ch2 Ch3 Ch4 Ch5 Ch3 Ch4

Plotted: 08:45 Dec 07, 2004

< EMI - ElectroMagnetic Instruments > 


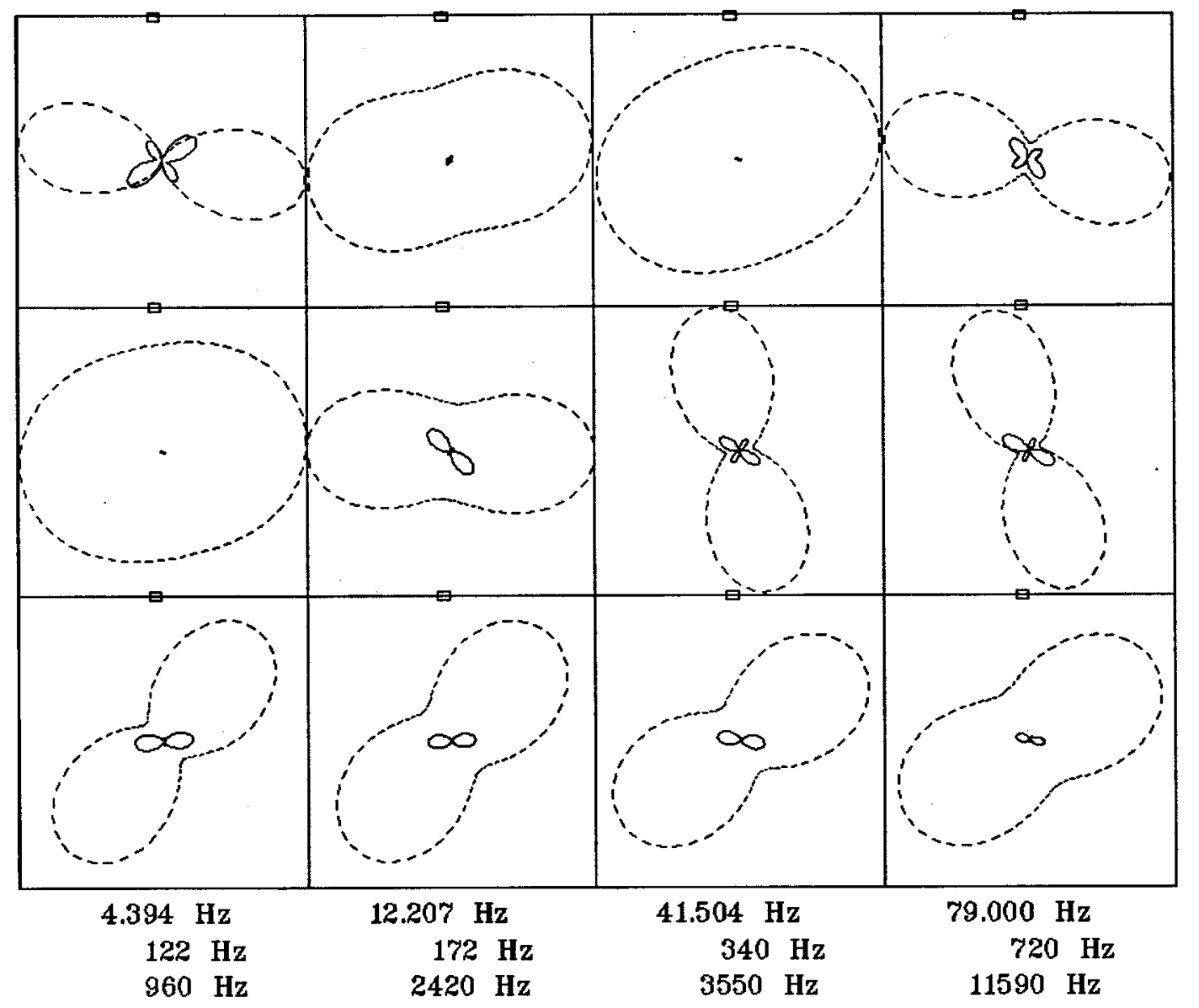

Rotation:

Client: Water Resources

Remote: none

Acquired: 11:0 Dec 05, 2003 Survey Co:USGS
Filename: nts5.avg

Channels: Ch1 Ch2 Ch3 Ch4 Ch5 Ch3 Ch4

Plotted: 08:45 Dec 07, 2004

$<$ EMI - ElectroMagnetic Instruments 


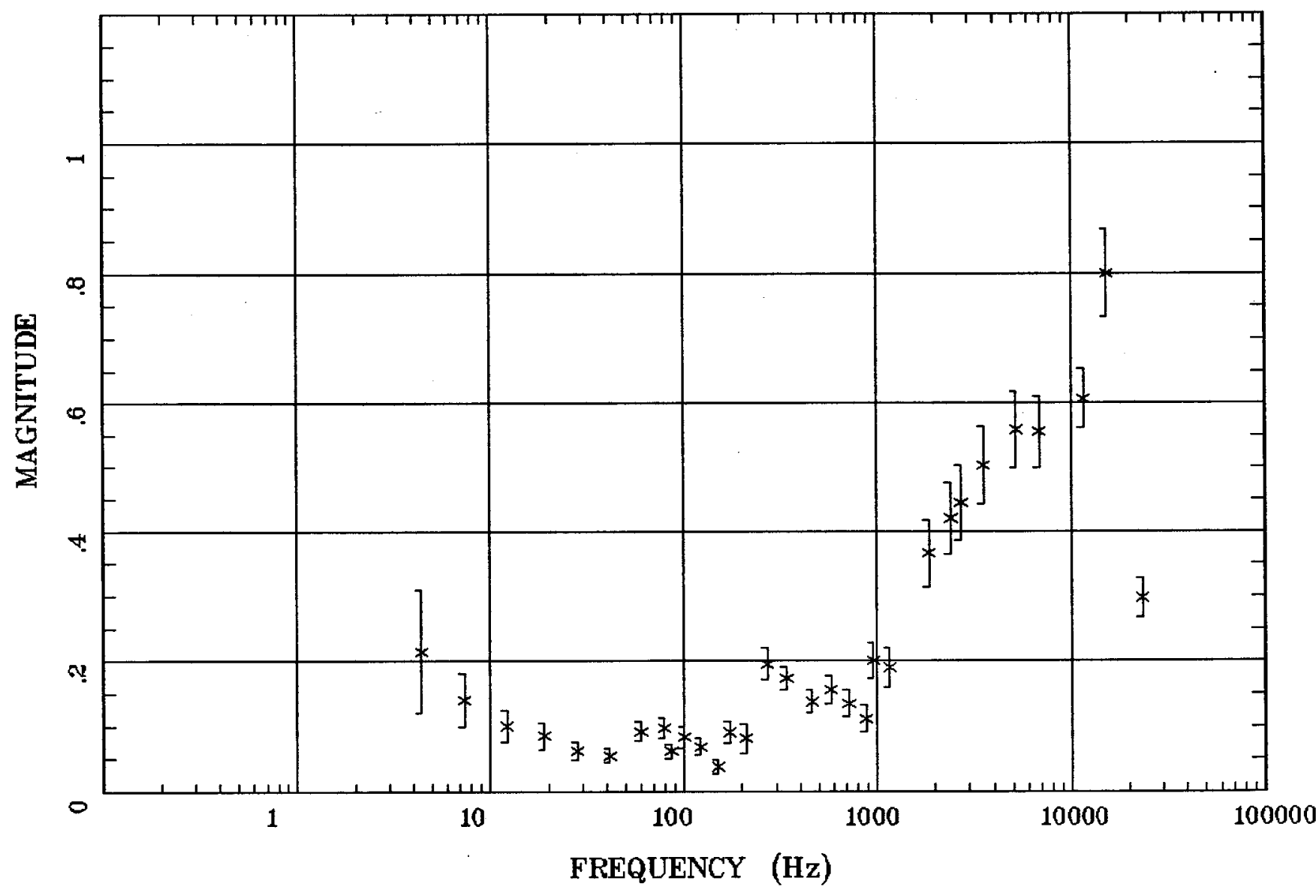

Client: Water Resources Remote: none Acquired: 11:0 Dec 05, 2003 Survey Co:USGS
Rotation:

Filename: nts5.avg

Channels: Ch1 Ch2 Ch3 Ch4 Ch5 Ch3 Ch4 Plotted: 08:45 Dec 07, 2004

< EMI - ElectroMagnetic Instruments > 


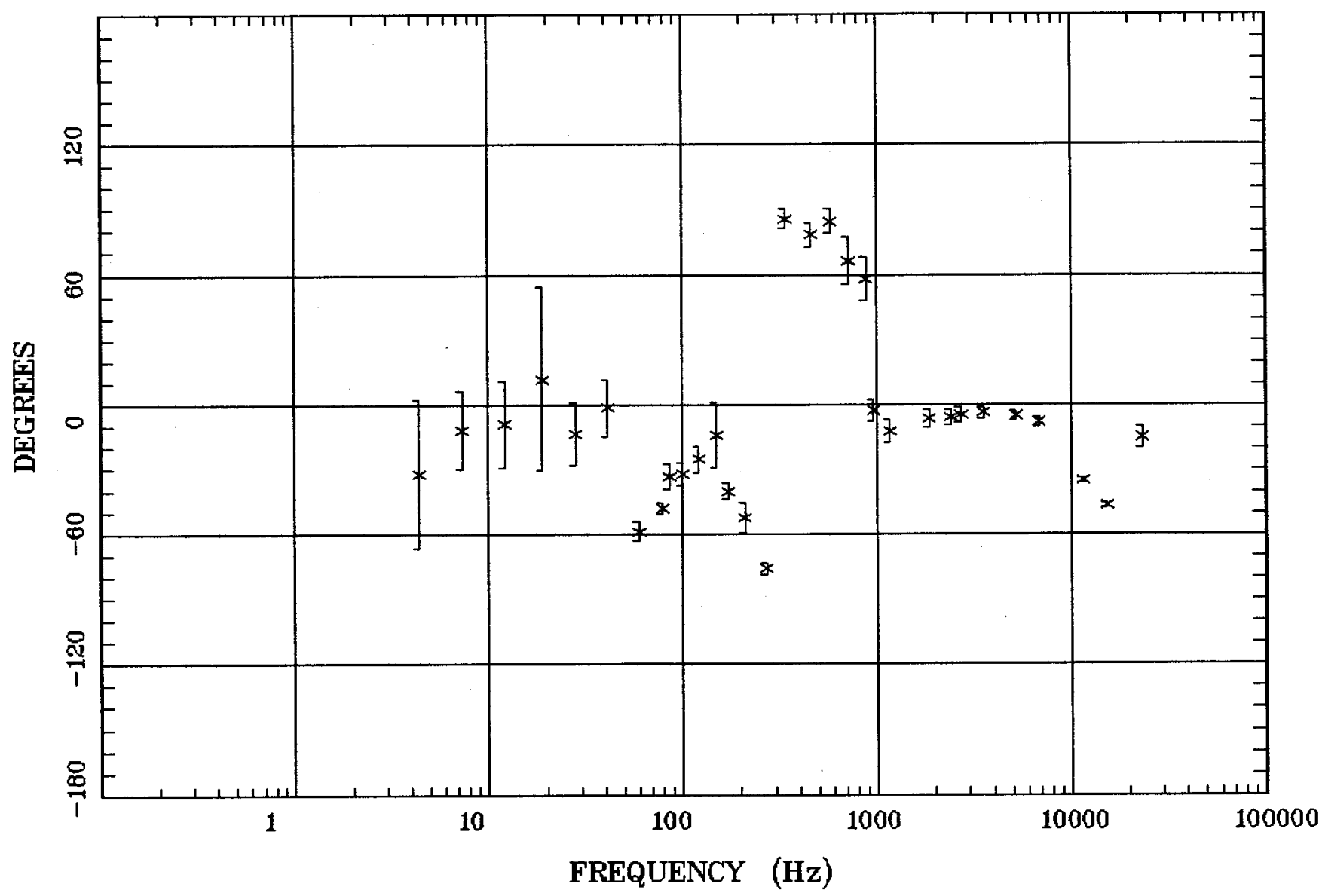

Client: Water Resources Remote: none Acquired: 11:0 Dec 05, 2003 Survey Co:USGS
Rotation:

Filename: nts5.avg

Channels: Ch1 Ch2 Ch3 Ch4 Ch5 Ch3 Ch4 Plotted: 08:45 Dec 07, 2004

< EMI - ElectroMagnetic Instruments > 
HzHx.x Coh HzHy.o

Priority 6

Station 5

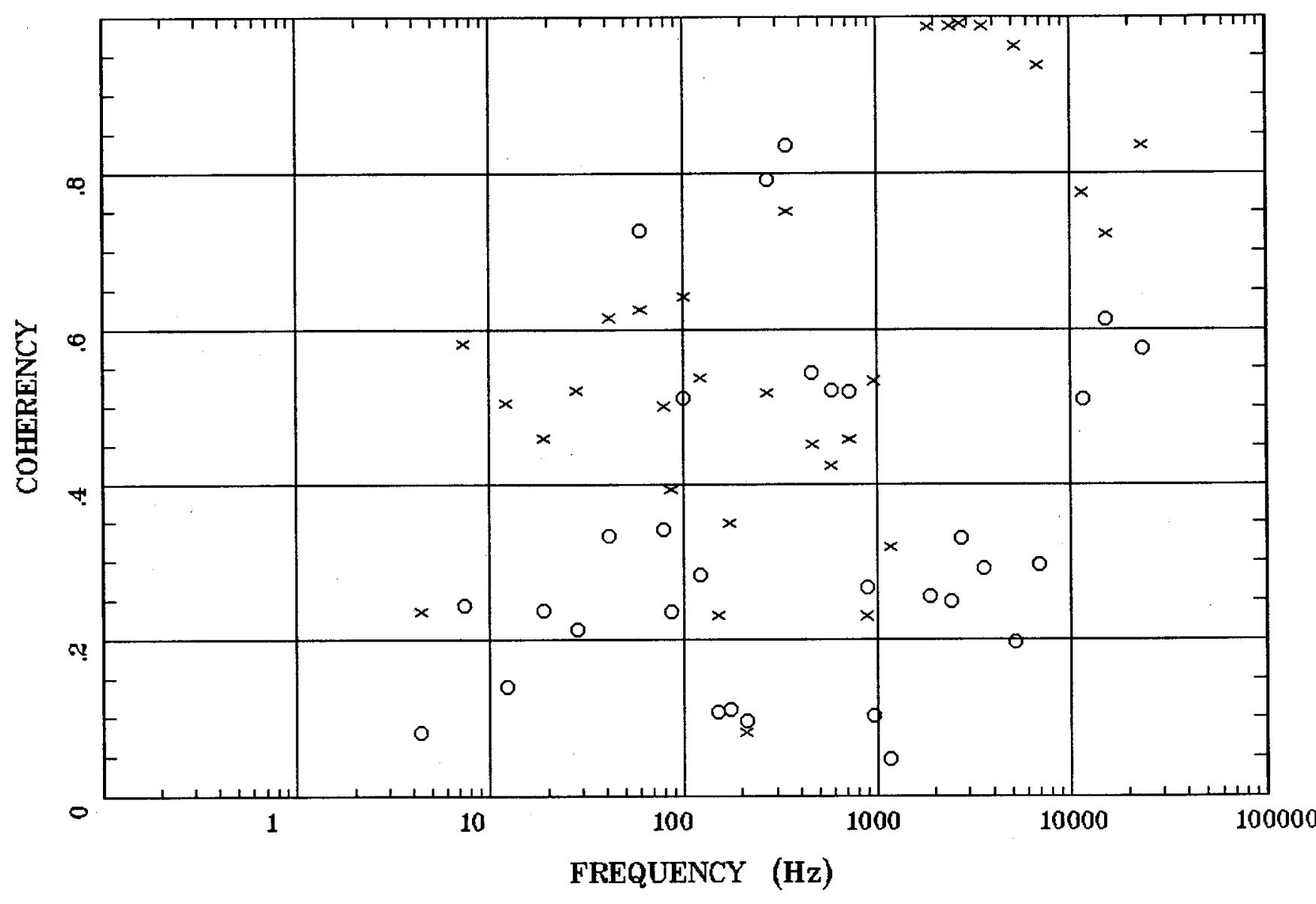

Client: Water Resources Remote: none

Acquired: 11:0 Dec 05, 2003 Survey co:USGS
Rotation:

Filename: nts5.avg

Channels: Ch1 Ch2 Ch3 ch4 Ch5 Ch3 Ch4 Plotted: 08:45 Dec 07, 2004

< EMI - ElectroMagnetic Instruments > 


\section{APPARENT RESISTIVITY}

Priority 6

Station 6

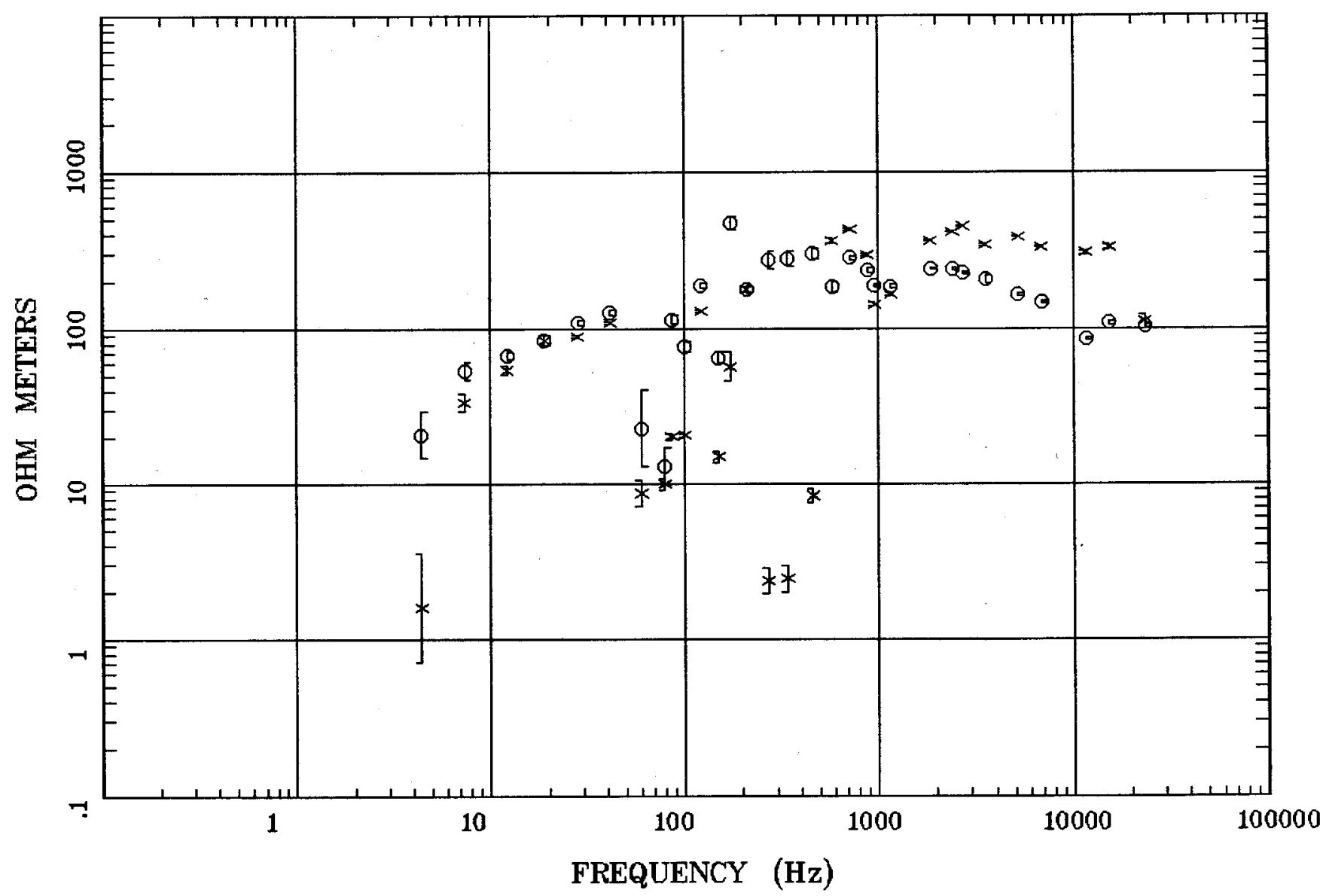

Client: Water Resources Remote: none

Acquired: 13:3 Dec 05, 2003 Survey Co:USGS
Rotation:

Filename: nts6.avg

Channels: Ch1 Ch2 ch3 ch4 Ch5 Ch3 Ch4 Plotted: 08:46 Dec 07, 2004

$<$ EMI - ElectroMagnetic Instruments 


\section{IMPEDANCE PHASE}

Priority 6

Station 6

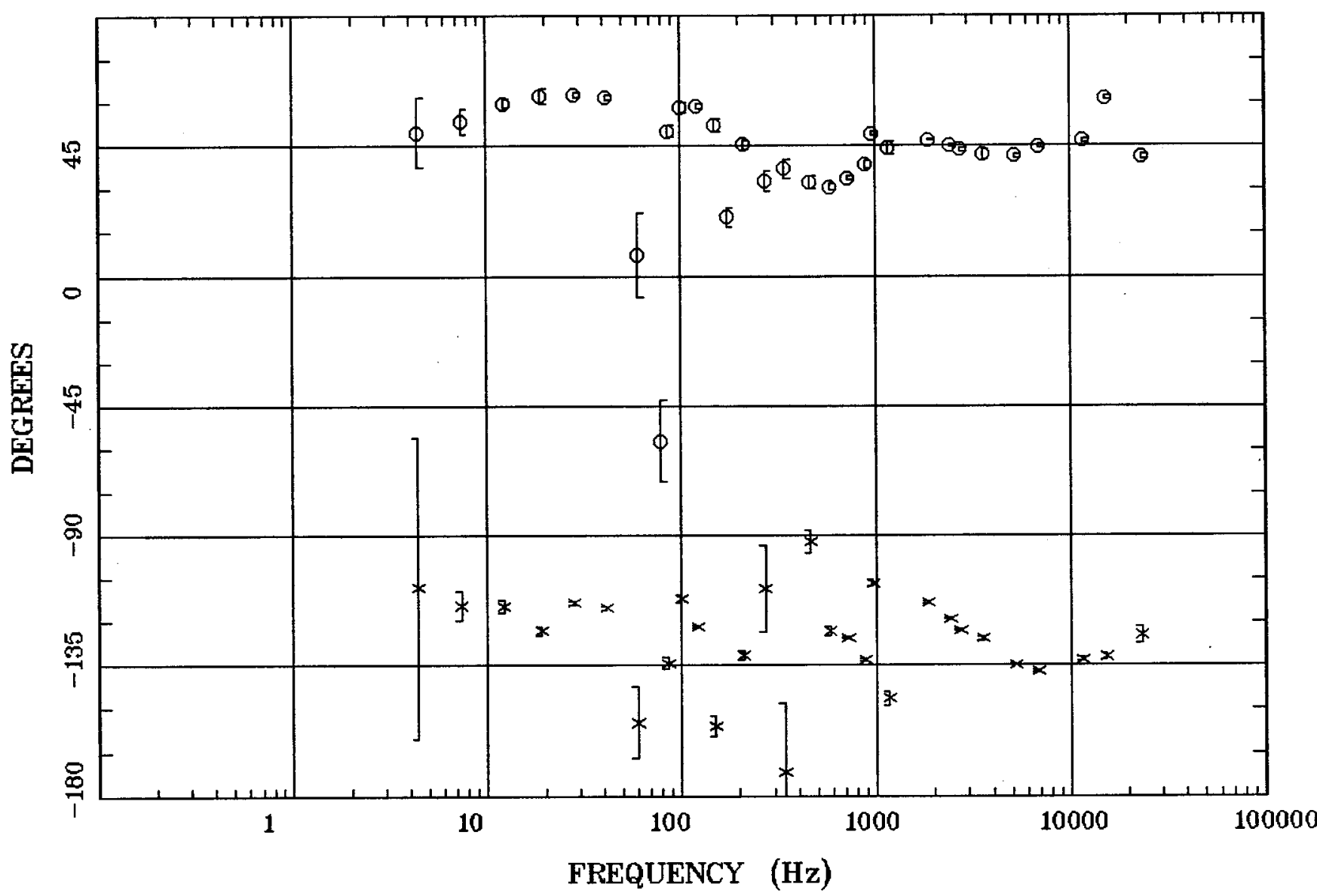

Client: Water Resources Remote: none Acquired: 13:3 Dec 05, 2003 Survey Co:USGS
Rotation:

Filename: nts6.avg

Channels: Ch1 Ch2 Ch3 Ch4 Ch5 Ch3 Ch4 Plotted: 08:46 Dec 07, 2004

< EMI - ElectroMagnetic Instruments 


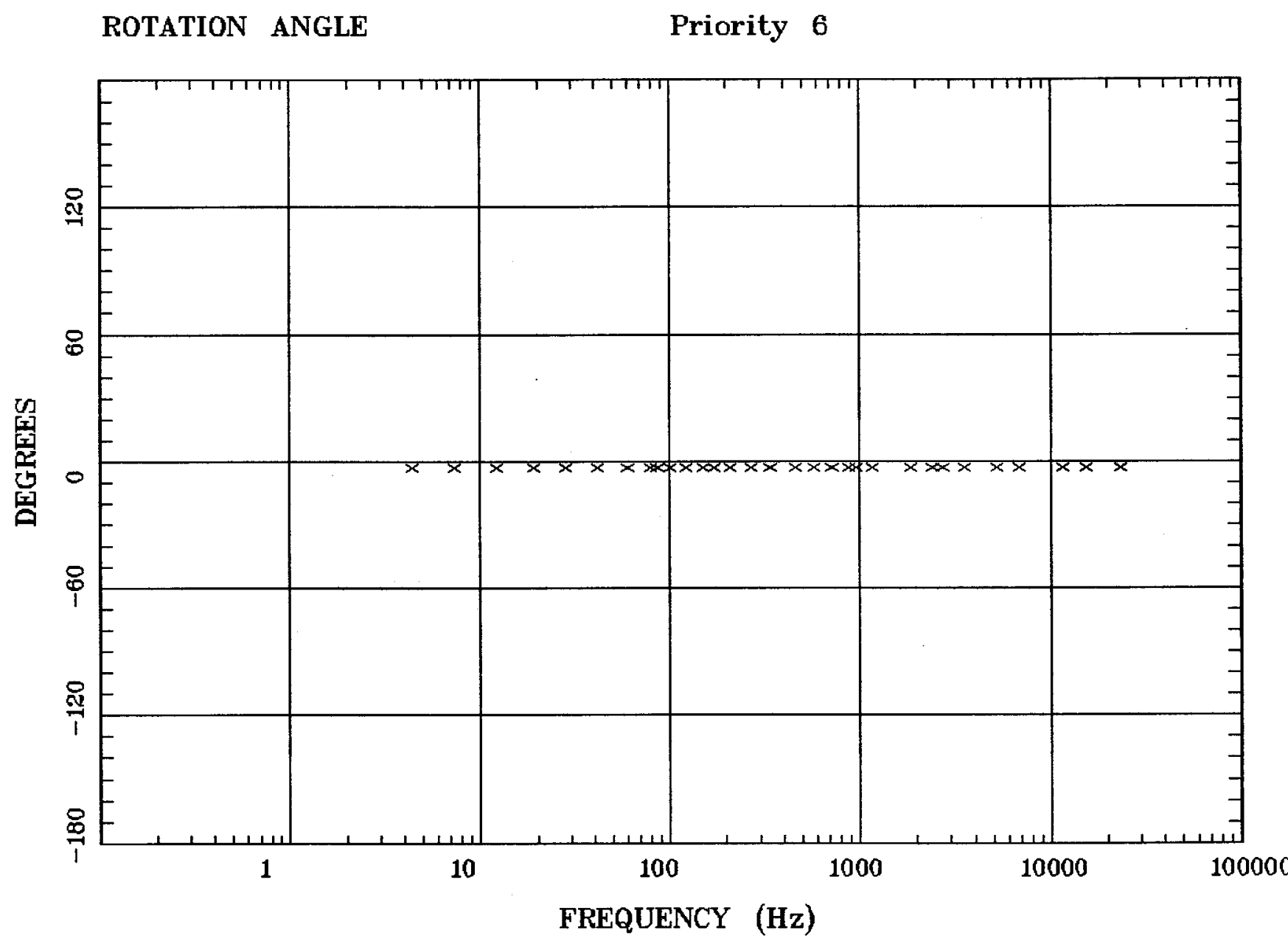

Client: Fater Resources Remote: none Acquired: 13:3 Dec 05, 2003 Survey Co:USGS

\section{Rotation:}

Filename: nts6.avg

Channels: Ch1 Ch2 Ch3 Ch4 Ch5 Ch3 Ch4 Plotted: 08:46 Dec 07, 2004

< EMI - ElectroMagnetic Instruments > 


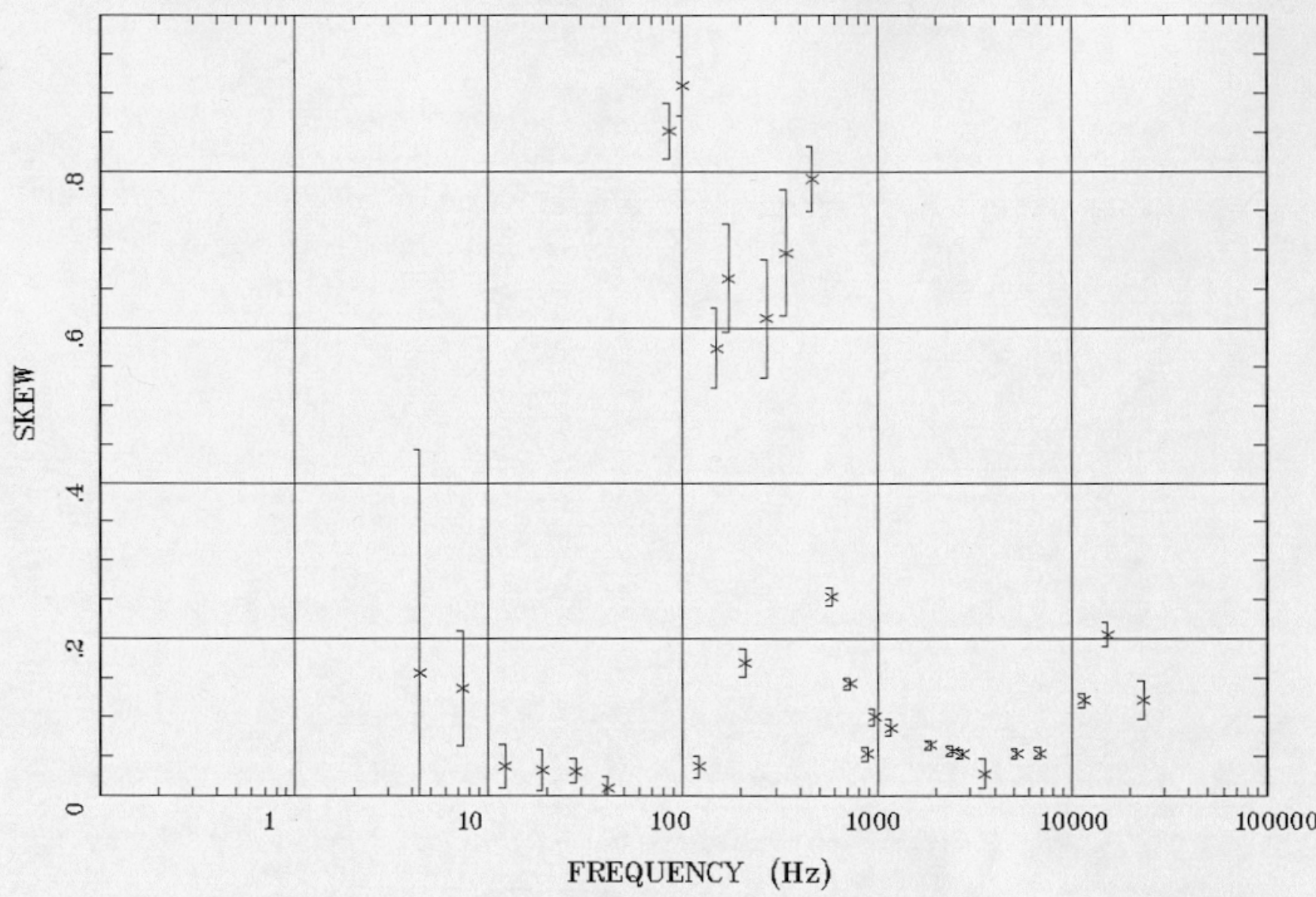

Client: Water Resources Remote: none Acquired: 13:3 Dec 05, 2003 Survey Co:USGS
Rotation:

Filename: nts6.avg

Channels: Ch1 Ch2 Ch3 Ch4 Ch5 Ch3 Ch4 Plotted: 08:46 Dec 0\%, 2004

< EMI - ElectroMagnetic Instruments > 


\section{E MULT Coh.}

Priority 6

Station 6

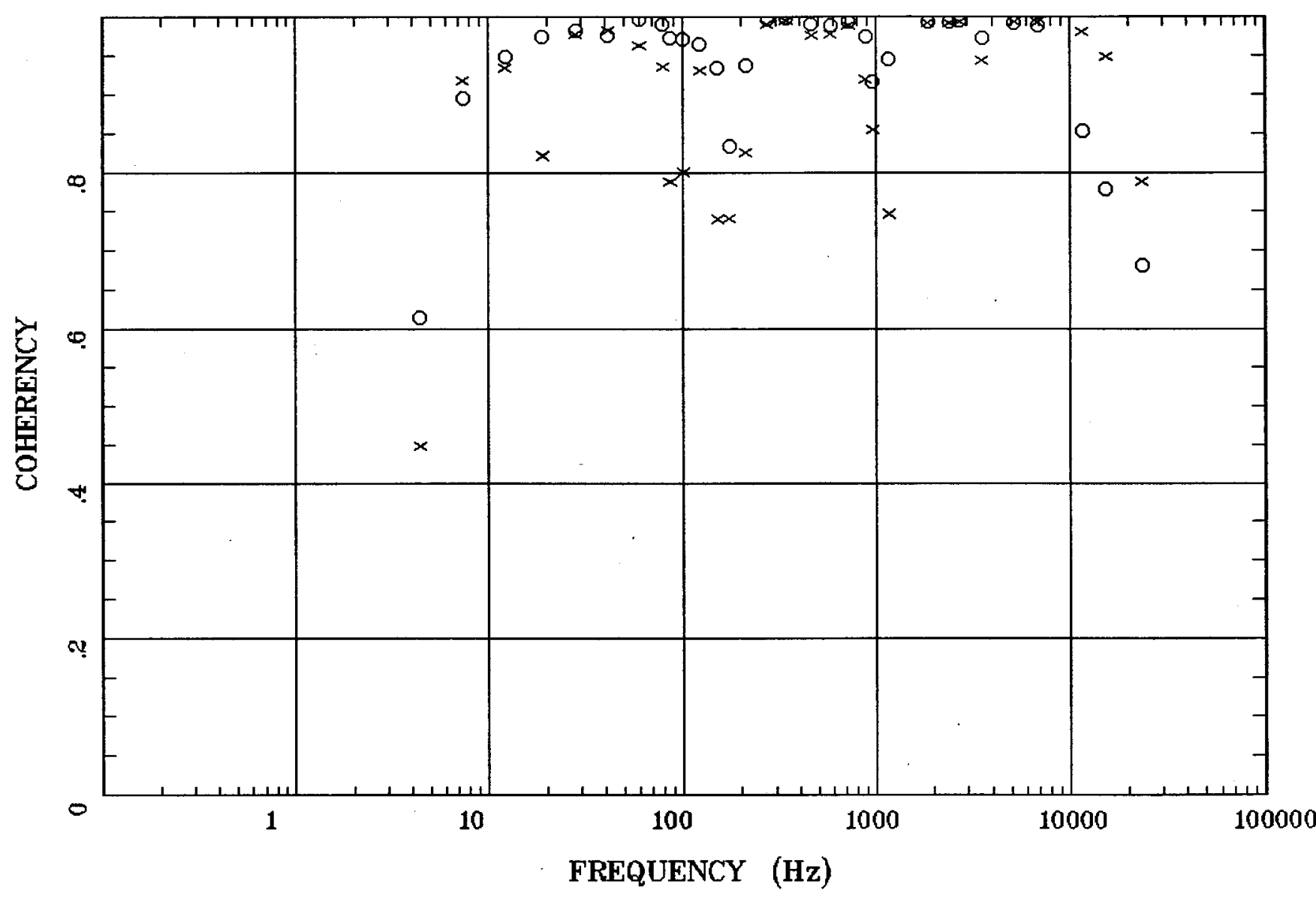

Client: Water Resources Remote: none Acquired: 13:3 Dec 05, 2003 Survey Co:USGS
Rotation:

Filename: nts6.avg

Channels: Ch1 Ch2 Ch3 Ch4 Ch5 Ch3 Ch4 Plotted: 08:46 Dec 07, 2004

< EMI - ElectroMagnetic Instruments > 


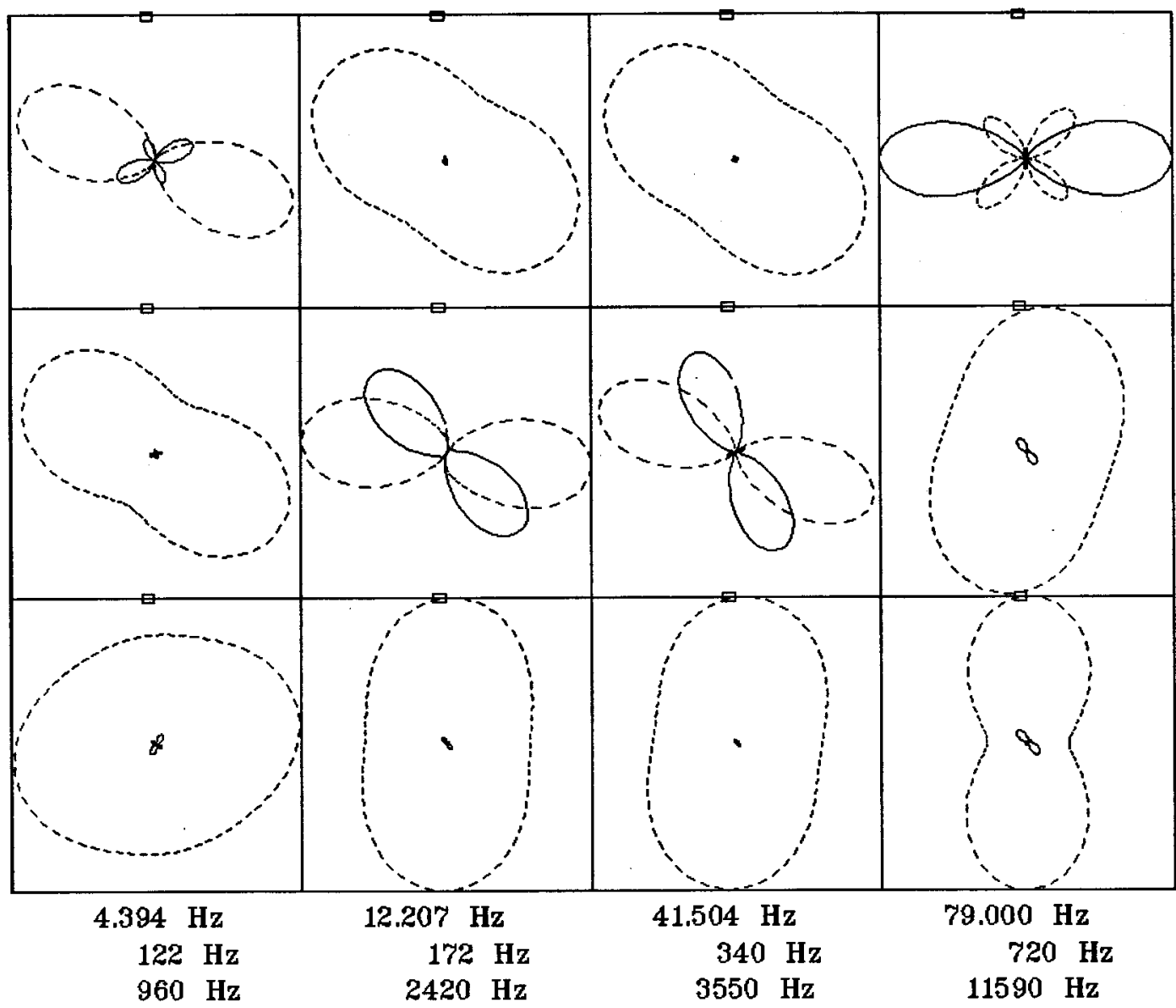

Client: Water Resources Remote: none Acquired: 13:3 Dec 05, 2003 Survey Co:USGS
Rotation:

Filename: nts6.avg Channels: Ch1 Ch2 Ch3 Ch4 Ch5 Ch3 Ch4 Plotted: 08:46 Dec 07, 2004

$<$ EMI - ElectroMagnetic Instruments > 


\section{TIPPER MAGNITUDE}

Priority 6

Station 6

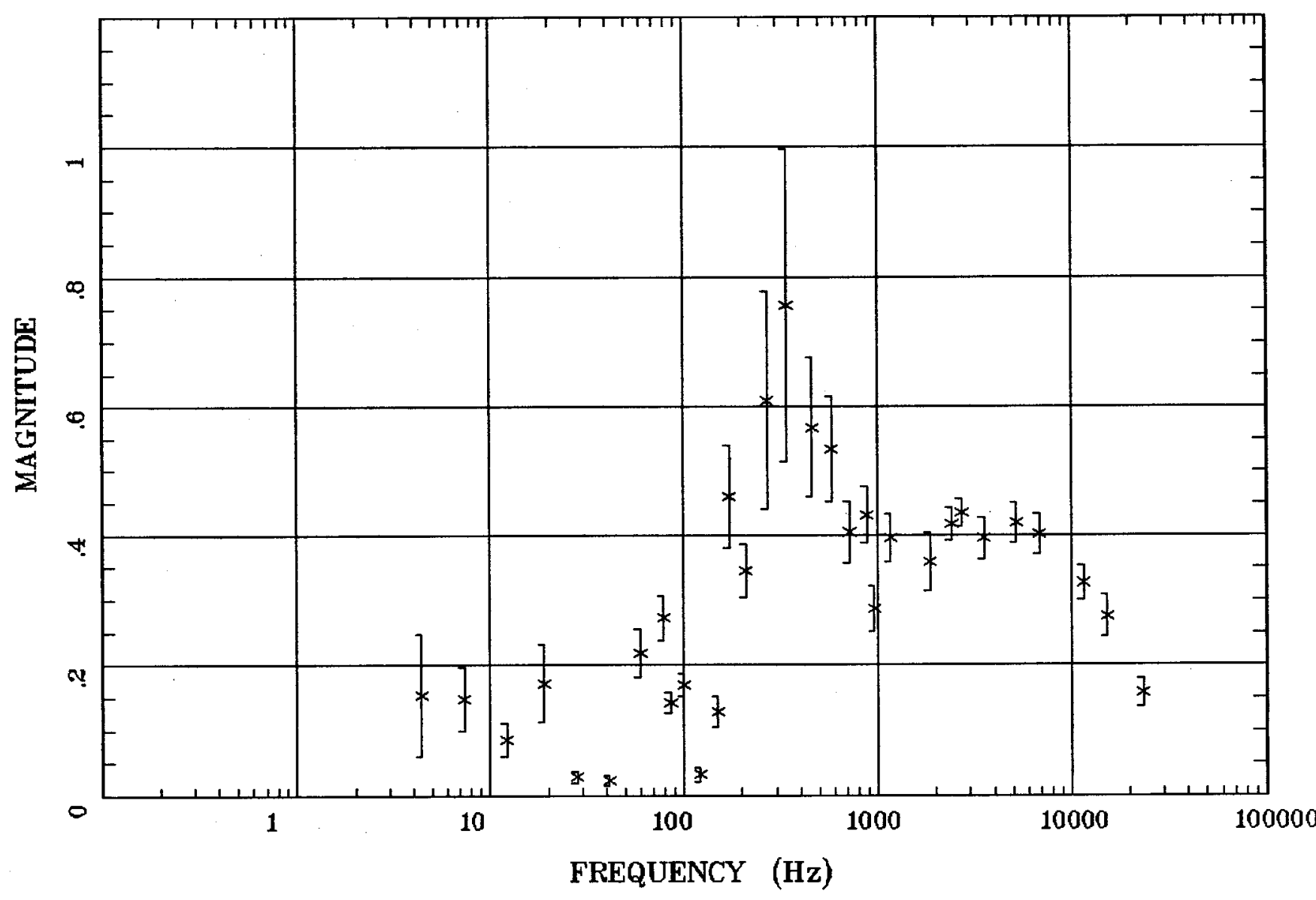

Client: Fater Resources Remote: none Acquired: 13:3 Dec 05, 2003 Survey Co:USGS
Rotation:

Filename: nts6.avg

Channels: Ch1 Ch2 Ch3 Ch4 Ch5 Ch3 Ch4 Platted: 08:46 Dec 07, 2004

< EMI - ElectroMagnetic Instruments 


\section{TIPPER STRIKE}

Priority 6

Station 6

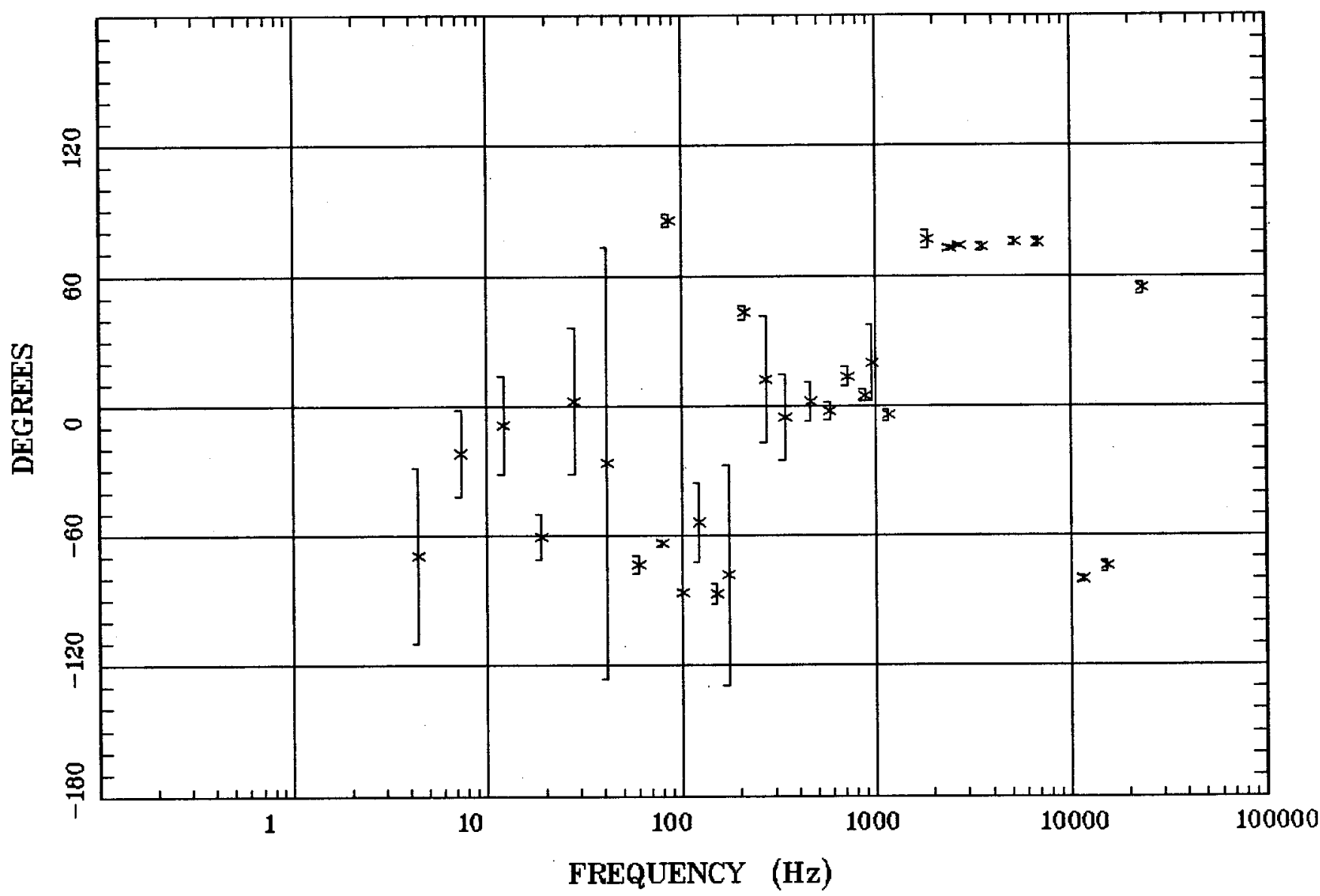

Client: Water Resources

Remote: none

Acquired: 13:3 Dec 05, 2003 Survey co:USGS
Rotation:

Filename: nts6.avg

Channels: Ch1 Ch2 Ch3 Ch4 Ch5 Ch3 Ch4 Plotted: 08:46 Dec 07, 2004

< EMI - ElectroMagnetic Instruments > 


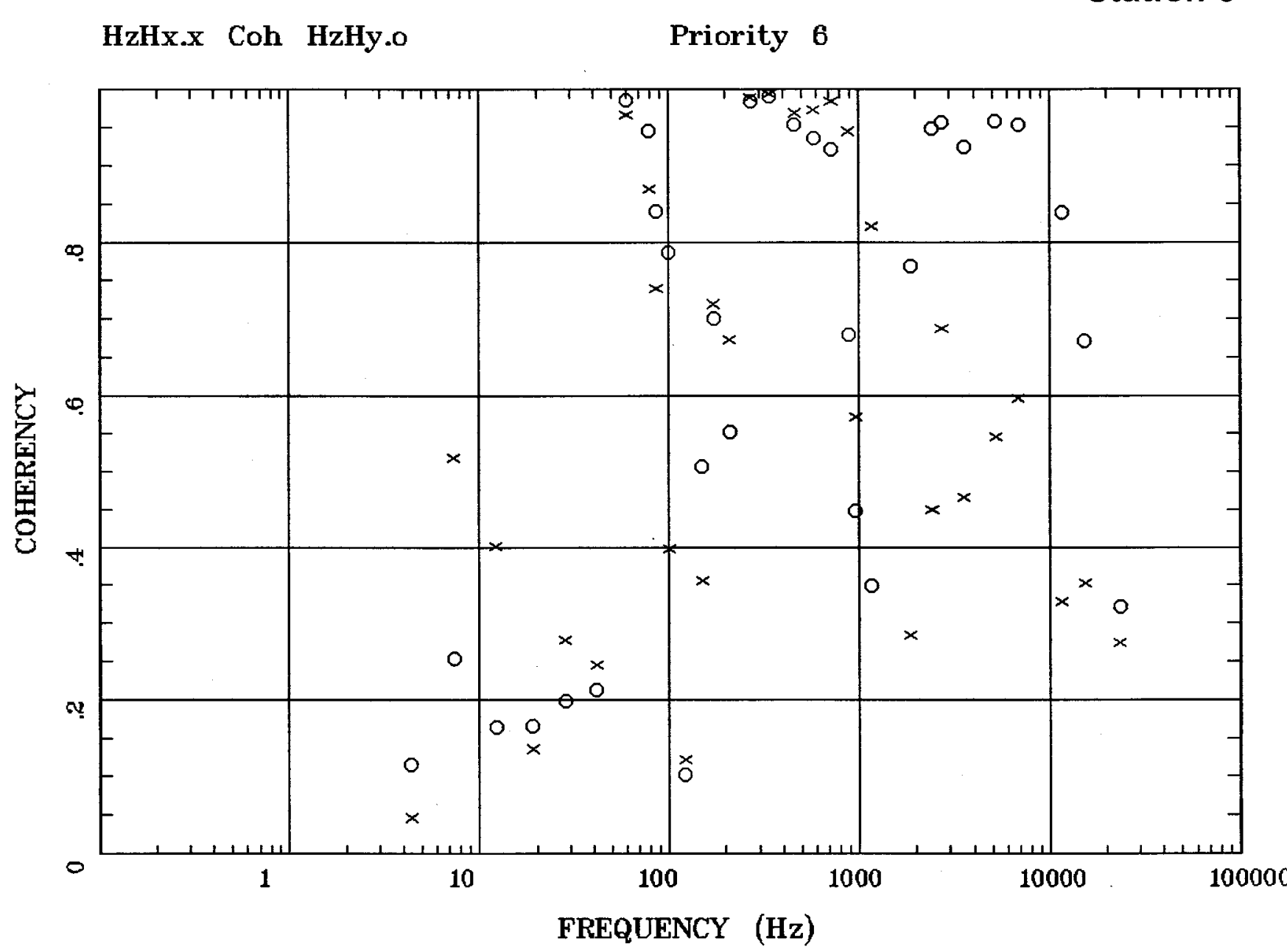

Client: Water Resources Remote: none Acquired: 13:3 Dec 05, 2003 Survey Co:USGS
Rotation:

Filen ame: nts6.avg

Channels: Ch1 Ch2 Ch3 Ch4 Ch5 Ch3 Ch4 Plotted: 08:46 Dec 07, 2004

< EMI - ElectroMagnetic Instruments 


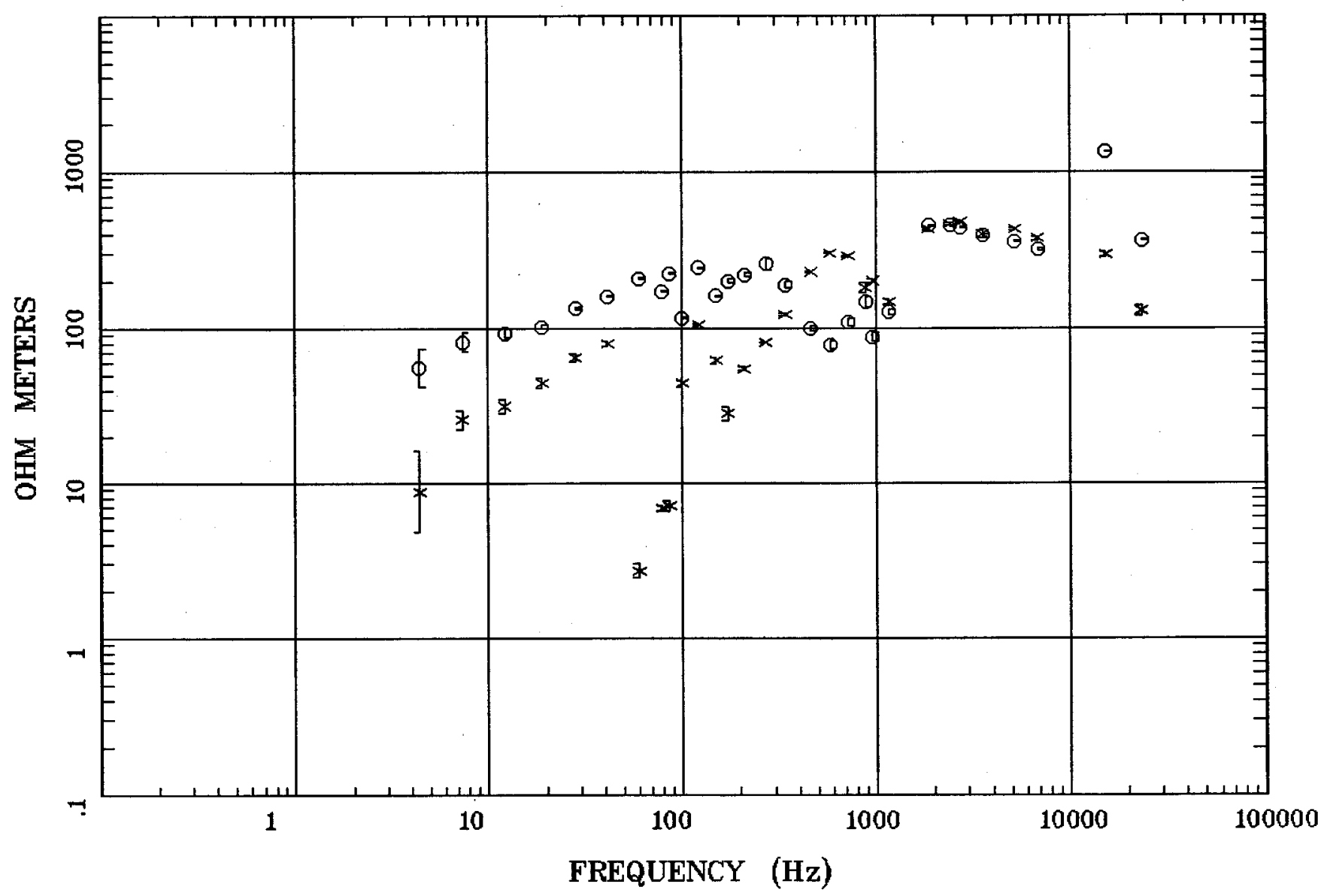

Client: Water Resources Remote: none Acquired: 14:3 Dec 03, 2003 Survey Co:USGS

\section{Rotation:}

Filename: nts7.avg

Channels: Ch1 Ch2 Ch3 Ch4 Ch5 Ch3 Ch4 Plotted: 08:46 Dec 07, 2004

< EMI - ElectroMagnetic Instruments > 


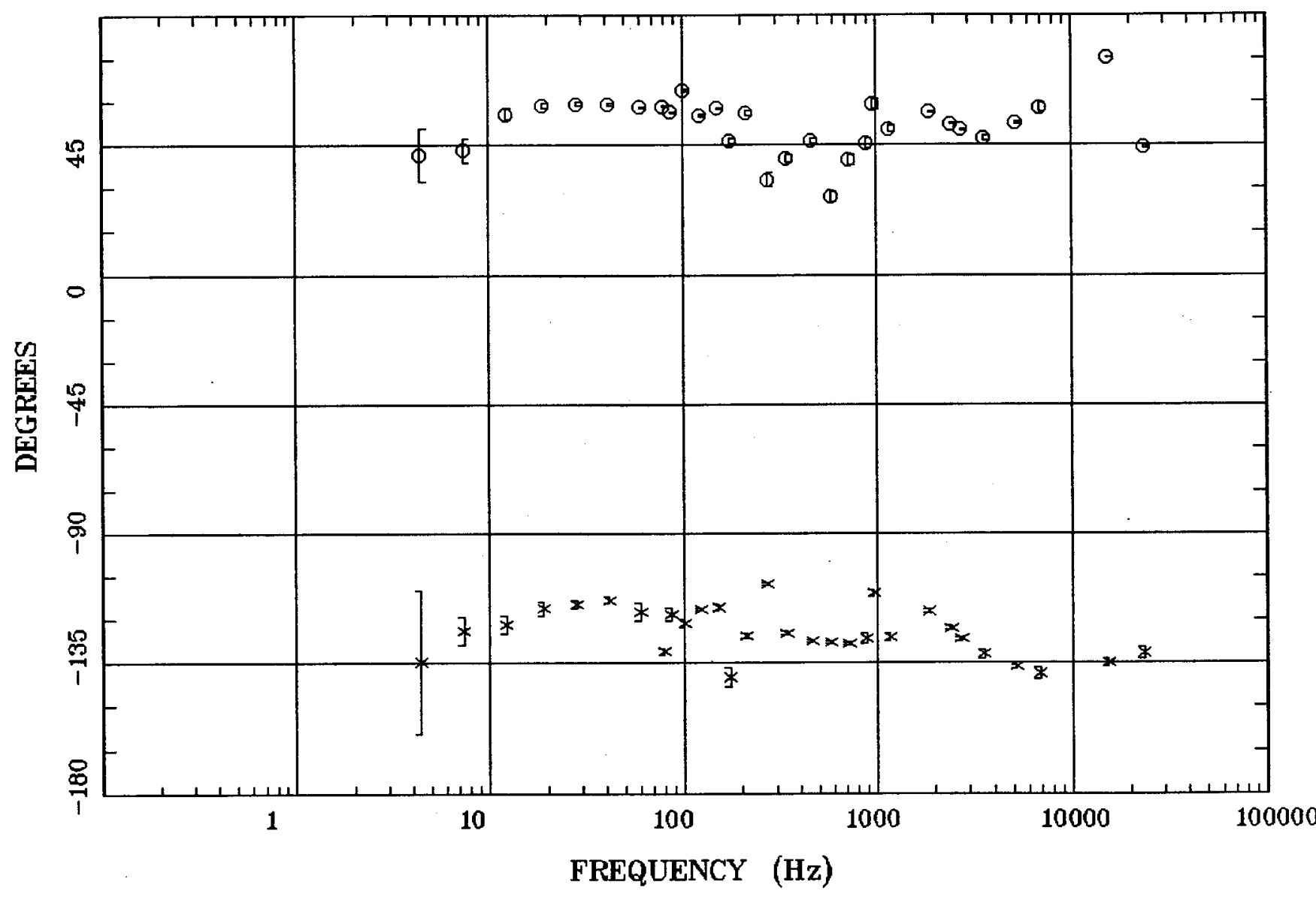

Client: Water Resources Remote: none Acquired: 14:3 Dec 03, 2003 Survey Co:USGS
Rotation:

Filename: nts7.avg

Channels: Ch1 Ch2 Ch3 Ch4 Ch5 Ch3 Ch4 Plotted: 08:46 Dec 07, 2004

< EMI - ElectroMagnetic Instruments > 


\section{ROTATION ANGLE}

\section{Priority 6}

Station 7

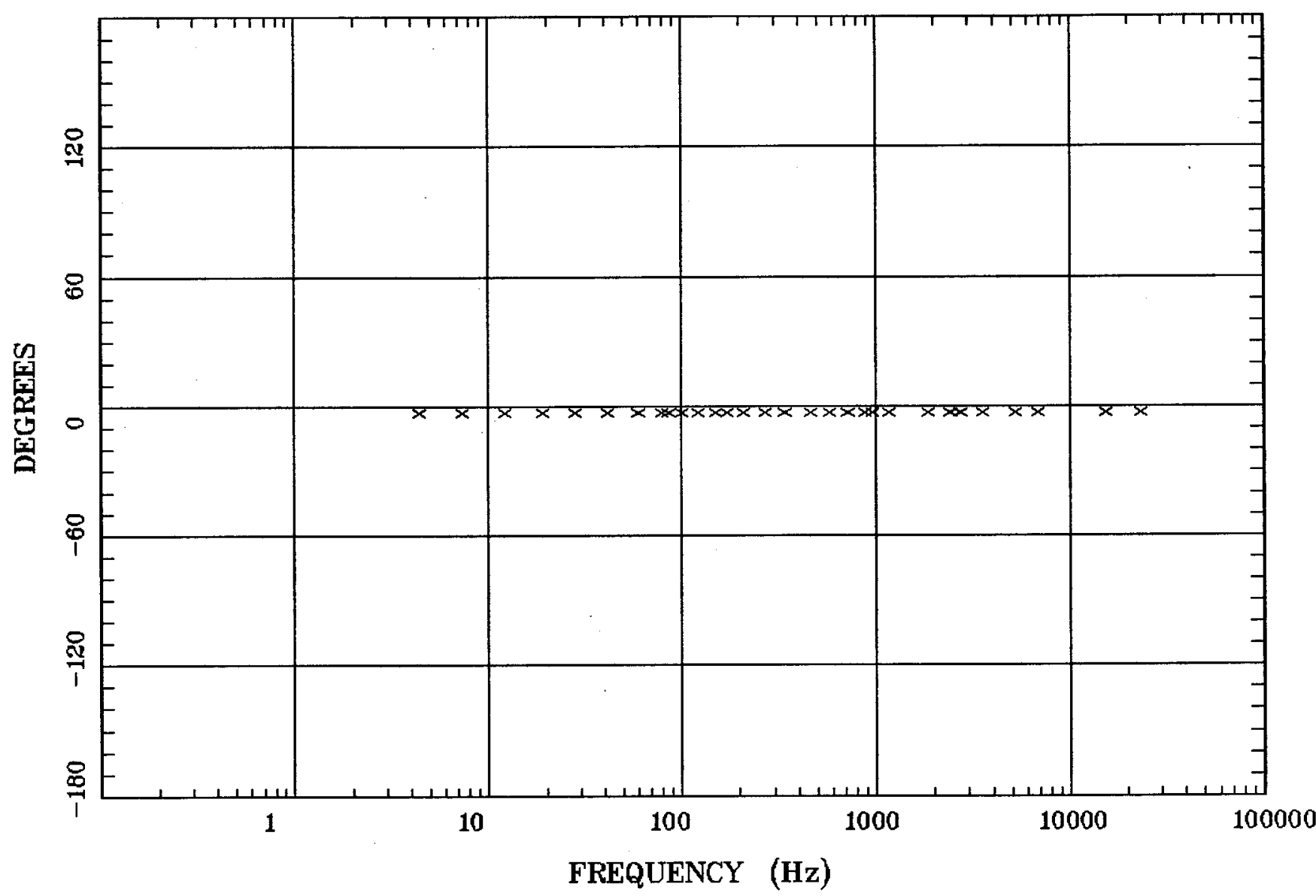

Client: Fater Resources Remote: none

Acquired: 14:3 Dec 03, 2003 Survey Co:USGS
Rotation:

Filename: nts7.avg

Channels: Ch1 Ch2 Ch3 Ch4 Ch5 Ch3 Ch4 Plotted: 08:46 Dec 07, 2004

< EMI - ElectroMagnetic Instruments > 


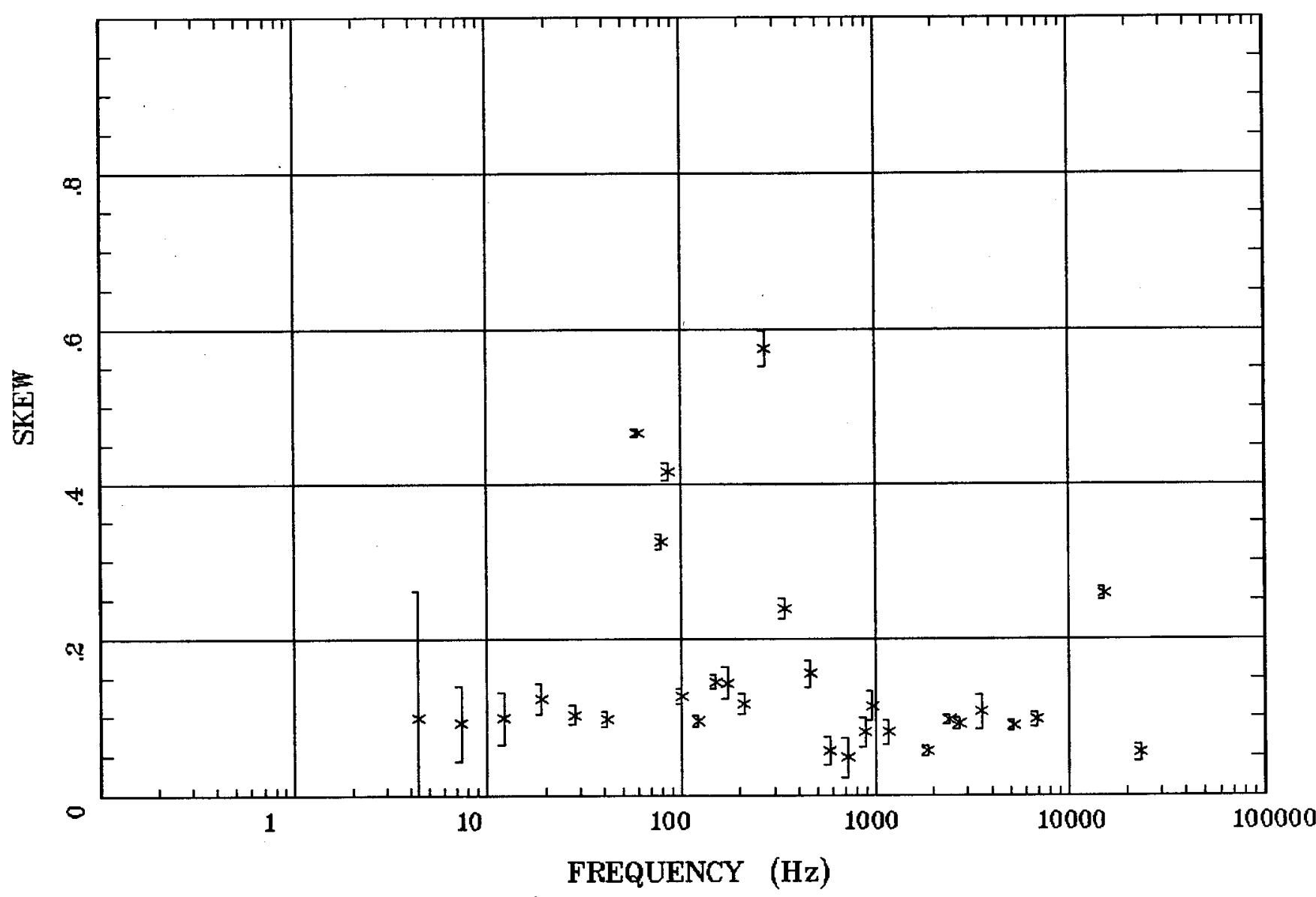

Client: Water Resources Remote: none

Acquired: 14:3 Dec 03, 2003 Survey Co:USGS
Rotation:

Filename: nts7.avg

Channels: Ch1 Ch2 Ch3 Ch4 Ch5 Ch3 Ch4 Plotted: 08:47 Dec 07, 2004

< EMI - ElectroMagnetic Instruments 


\section{E MULT Coh.}

Priority 6

Station 7

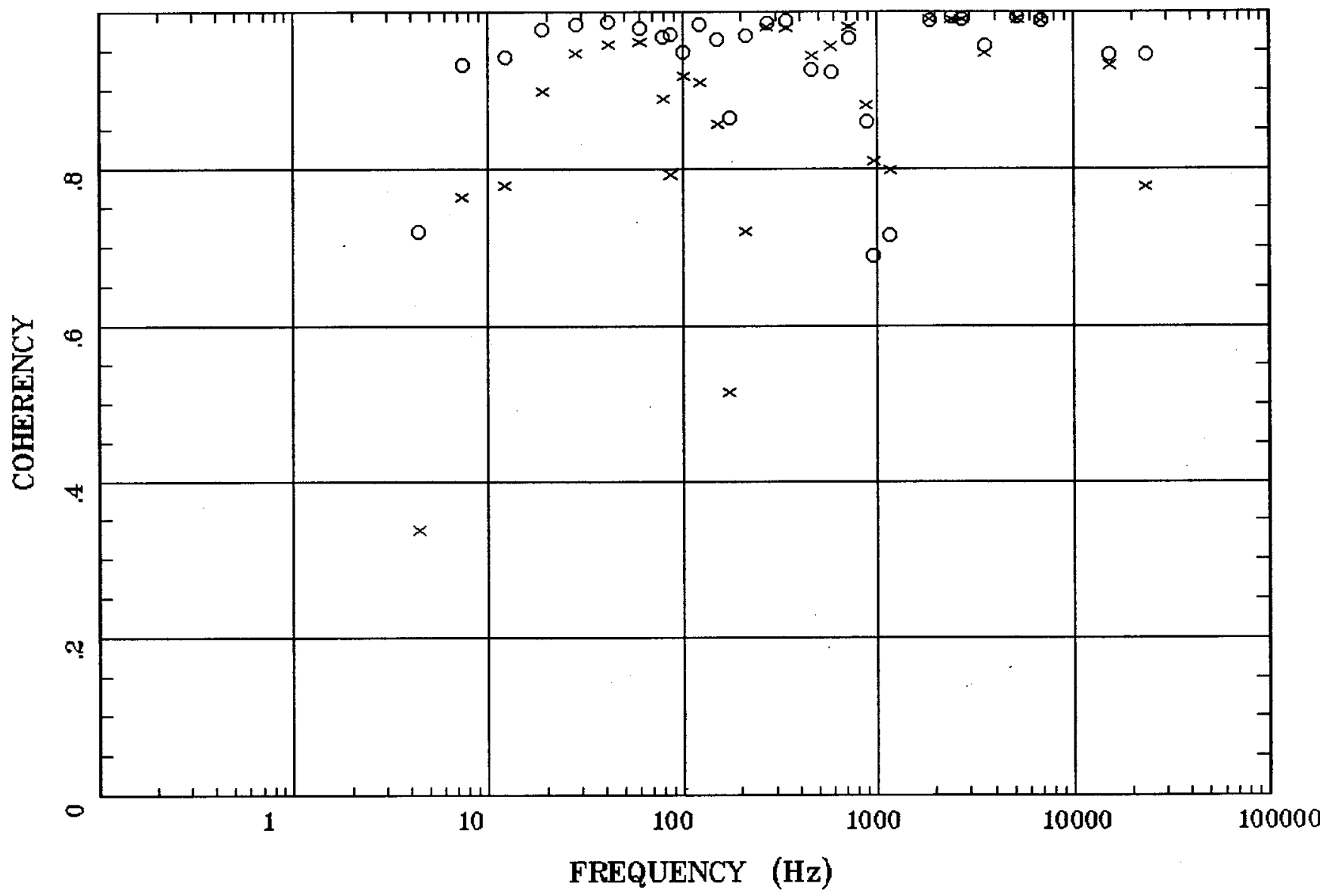

Client: Water Resources Remote: none Acquired: 14:3 Dec 03, 2003 Survey Co:USGS
Rotation:

Filename: nts7.avg

Channels: Ch1 Ch2 Ch3 Ch4 Ch5 Ch3 Ch4 Plotted: 08:47 Dec 07, 2004

$<$ EMI - ElectroMagnetic Instruments 


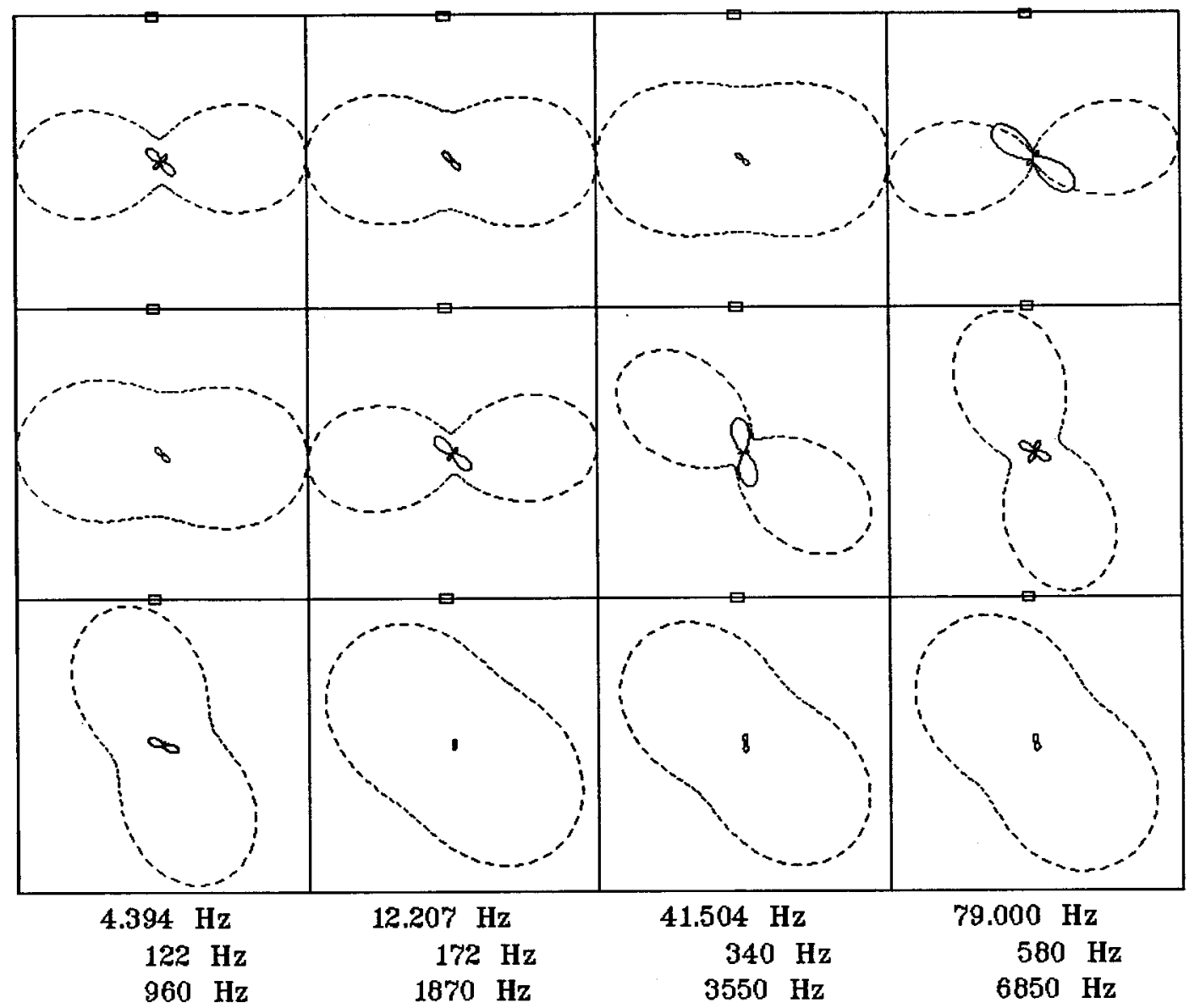

Client: Water Resources

Remote: none

Acquired: 14:3 Dec 03, 2003 Survey Co:USGS
Rotation:

Filename: nts7.avg

Channels: Ch1 Ch2 Ch3 Ch4 Ch5 Ch3 Ch4

Plotted: 08:47 Dec 07, 2004

$<$ EMI - ElectroMagnetic Instruments > 


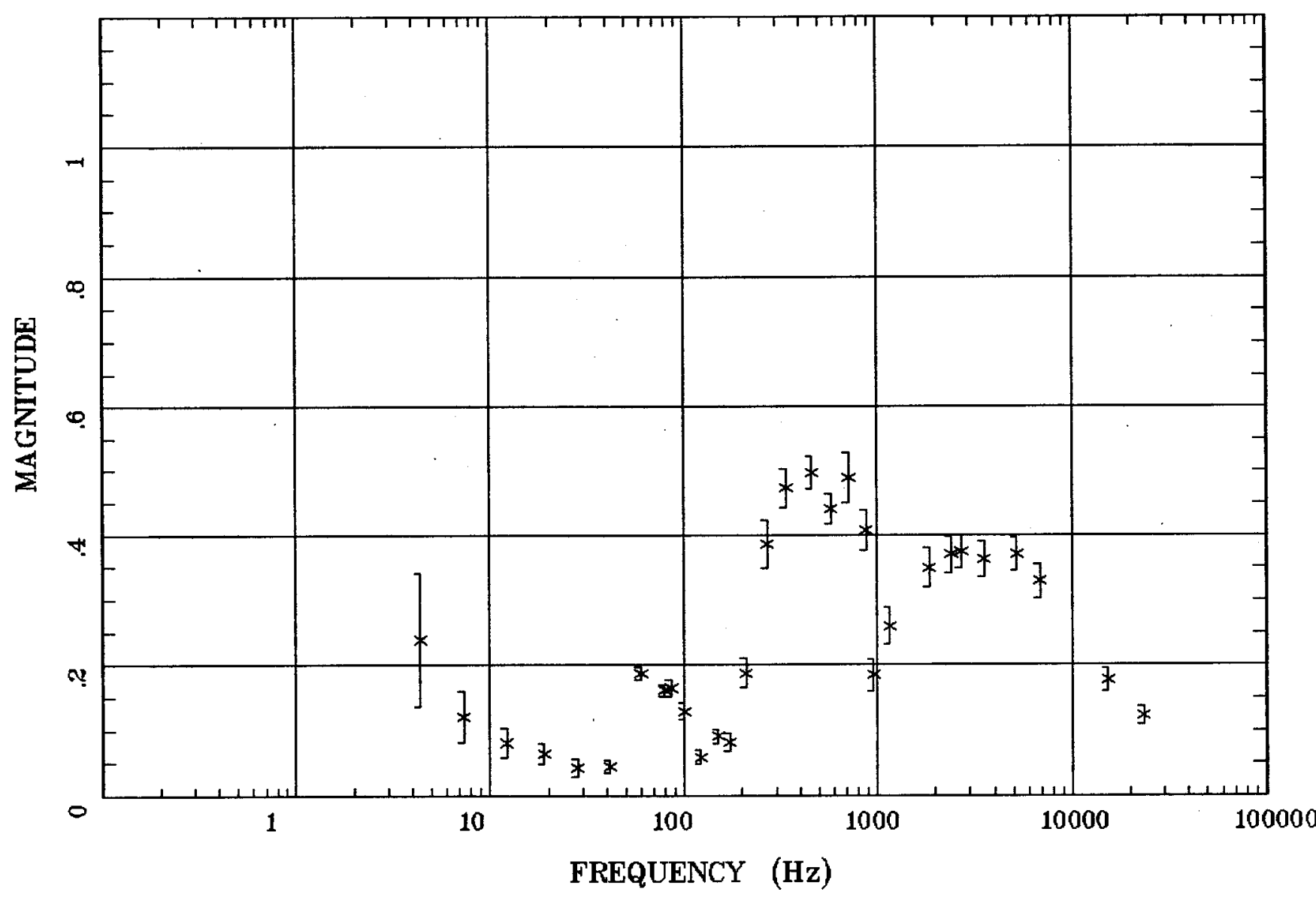

Client: Water Resources

Remote: none

Acquired: 14:3 Dec 03, 2003 Survey Co:USGS
Rotation:

Filename: nts7.avg

Channels: Ch1 Ch2 Ch3 Ch4 Ch5 Ch3 Ch4 Platted: 08:47 Dec 07, 2004

$<$ EMI - ElectroMagnetic Instruments 


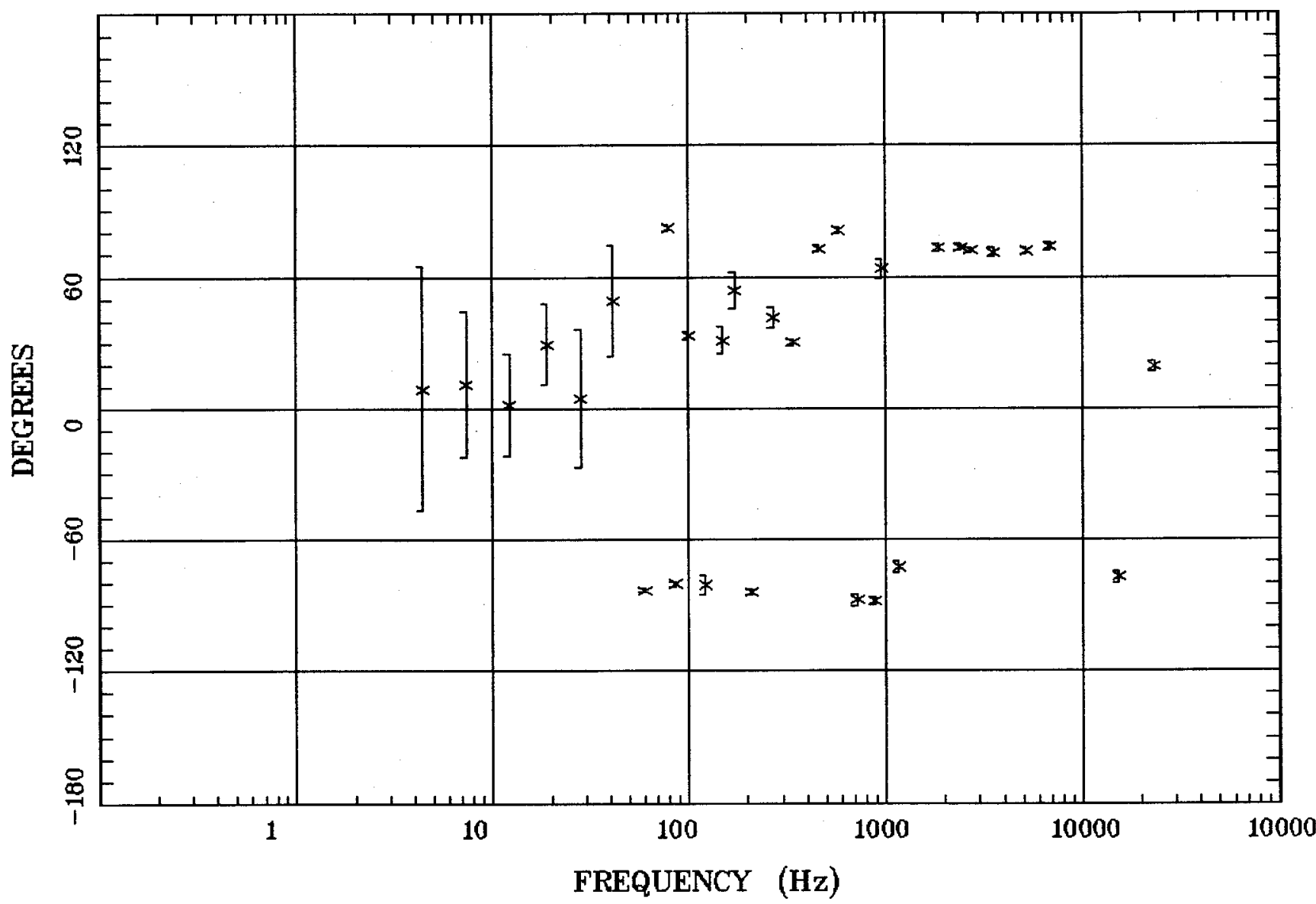

Client: Water Resources Remote: none Acquired: 14:3 Dec 03, 2003 Survey Co:USGS
Rotation:

Filename: nts7.avg

Channels: Ch1 Ch2 Ch3 Ch4 Ch5 Ch3 Ch4 Platted: 08:47 Dec 07, 2004

< EMI - ElectroMagnetic Instruments > 


\section{HzHx.x Coh HzHy.o}

\section{Priority 6}

Station 7

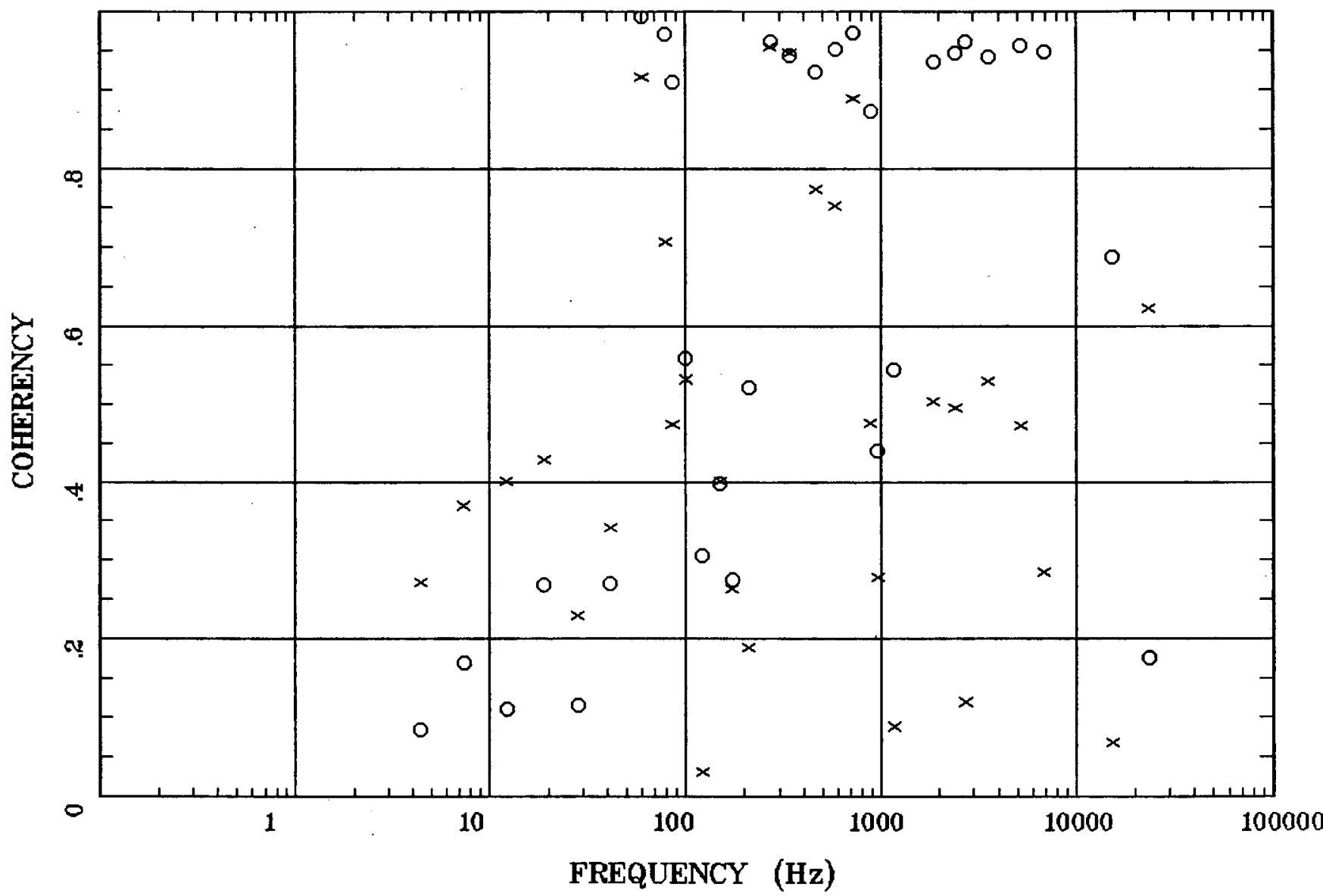

Client: Water Resources Remote: none

Acquired: 14:3 Dec 03, 2003 Survey Co:USGS
Rotation:

Filename: nts\%.avg

Channels: Ch1 Ch2 Ch3 Ch4 Ch5 Ch3 Ch4 Plotted: 08:47 Dec 07, 2004

< EMI - ElectroMagnetic Instruments 


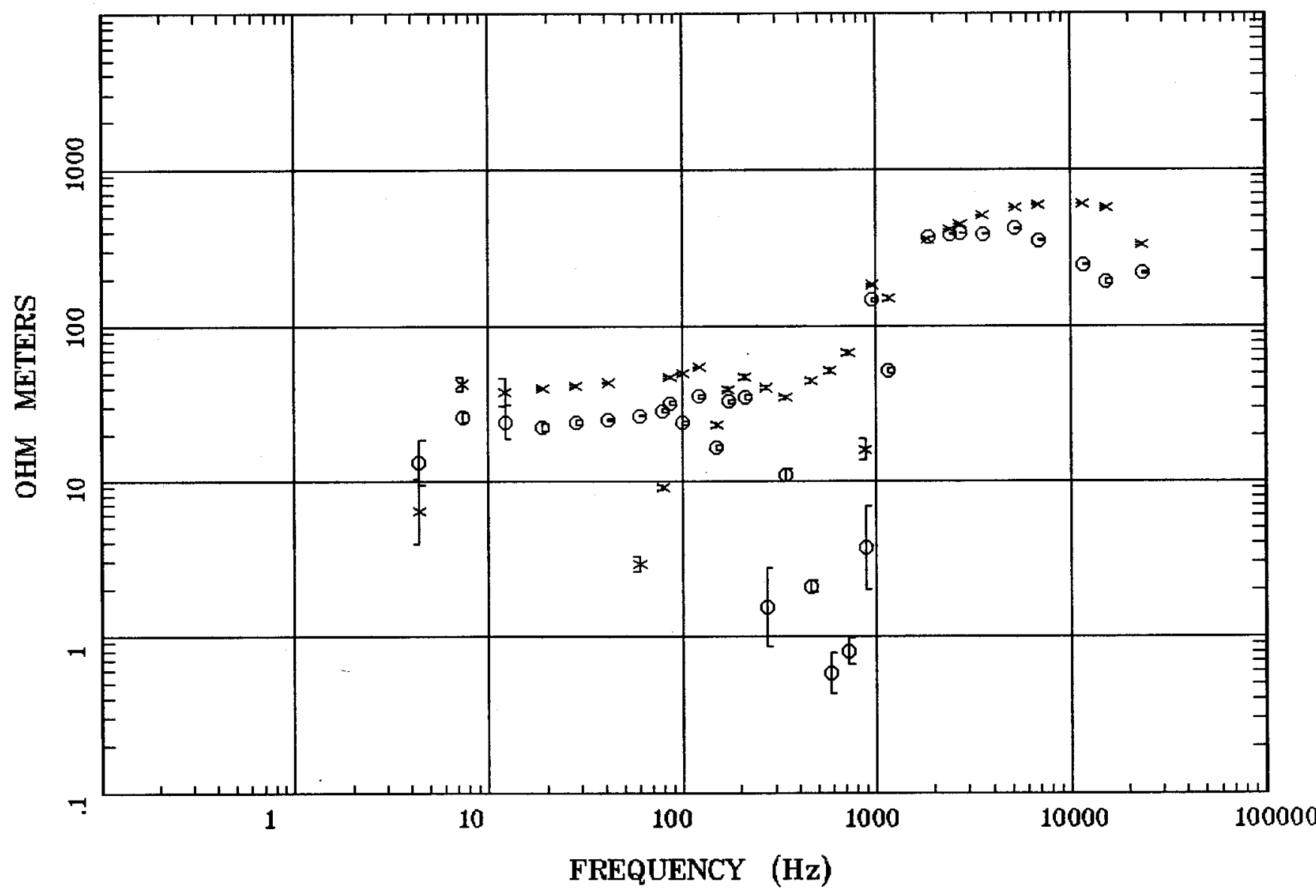

Client: Tater Resources Remote: none Acquired: 11:1 Dec 03, 2003 Survey Co:USGS
Rotation:

Filename: nts8.avg

Channels: Ch1 Ch2 Ch3 Ch4 Ch5 Ch3 Ch4 Plotted: 08:47 Dec 07, 2004

$<$ EMI - ElectroMagnetic Instruments 


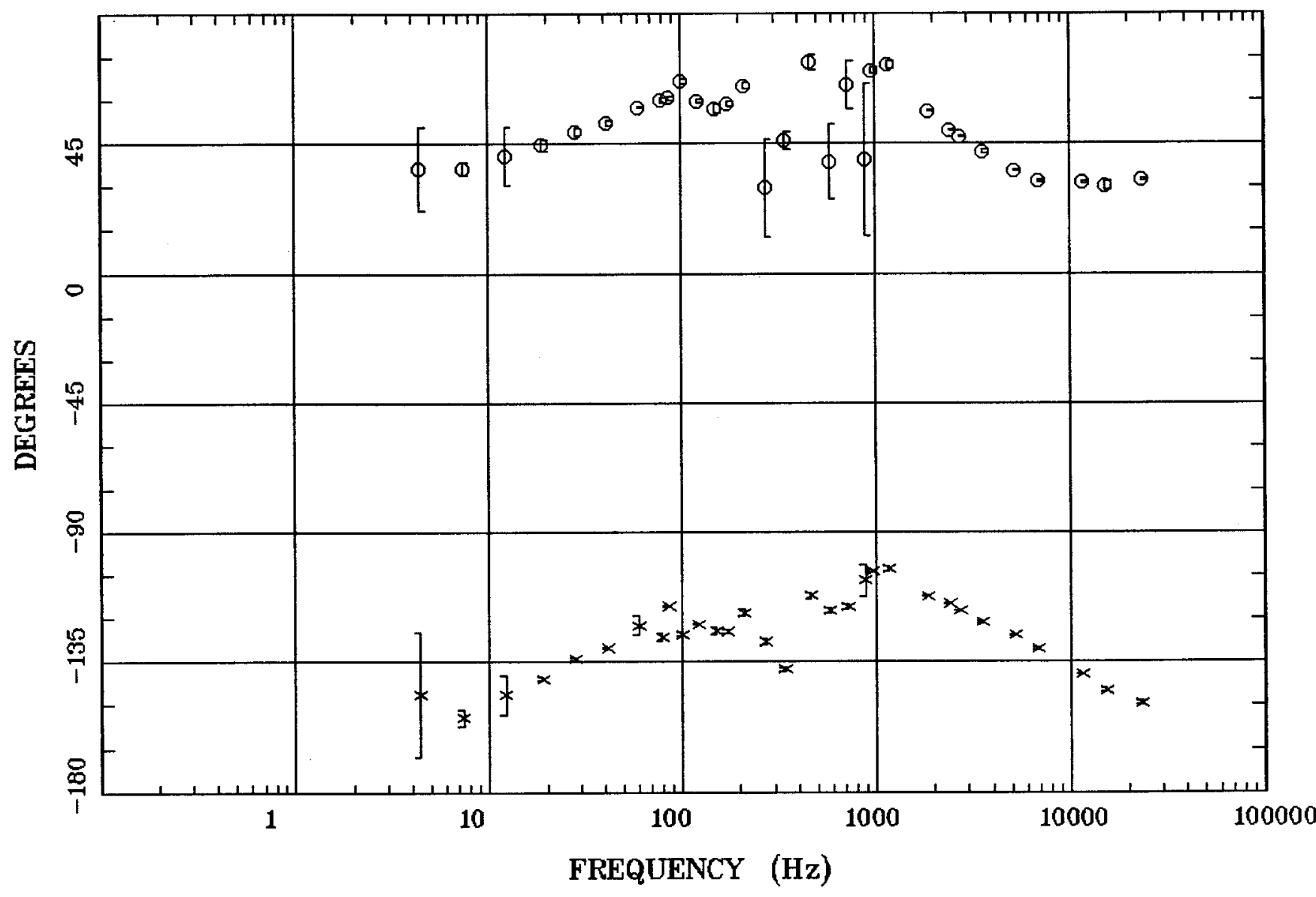

Client: Water Resources Remote: none Acquired: 11:1 Dec 03, 2003 Survey Co:USGS
Rotation:

Filename: nts6.avg

Channels: Ch1 Ch2 Ch3 Ch4 Ch5 Ch3 Ch4 Plotted: 08:47 Dec 07, 2004

<EMI - ElectroMagnetic Instruments > 


\section{ROTATION ANGLE}

Priority 6

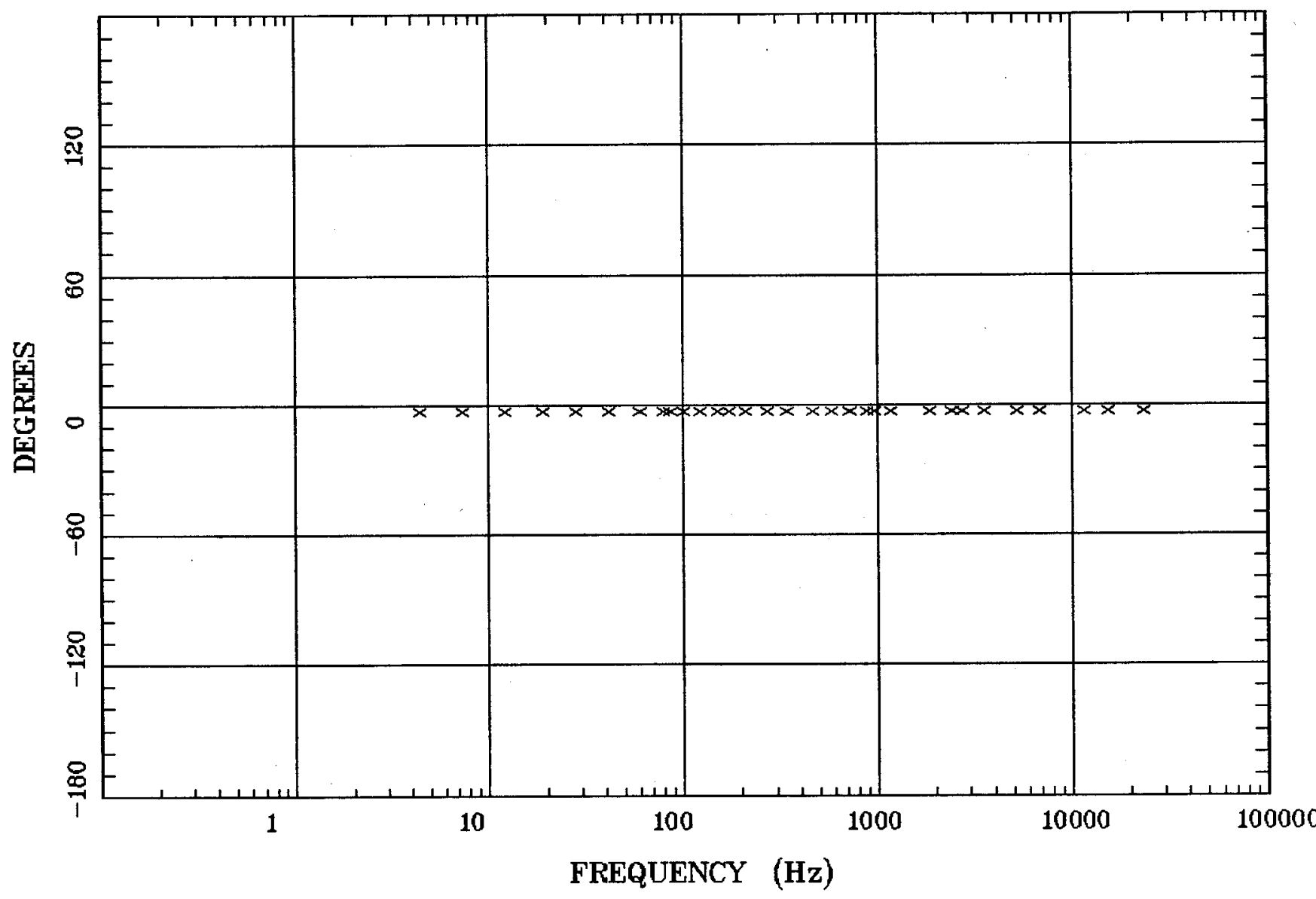

Client: Water Resources Remote: none Acquired: 11:1 Dec 03, 2003 Survey Co:USGS

\section{Rotation:}

Filename: nts8.avg

Channels: Ch1 Ch2 Ch3 Ch4 Ch5 Ch3 Ch4

Plotted: 08:47 Dec 07, 2004

< EMI - ElectroMagnetic Instruments > 


\section{IMPEDANCE SKEW}

Priority 6

Station 8

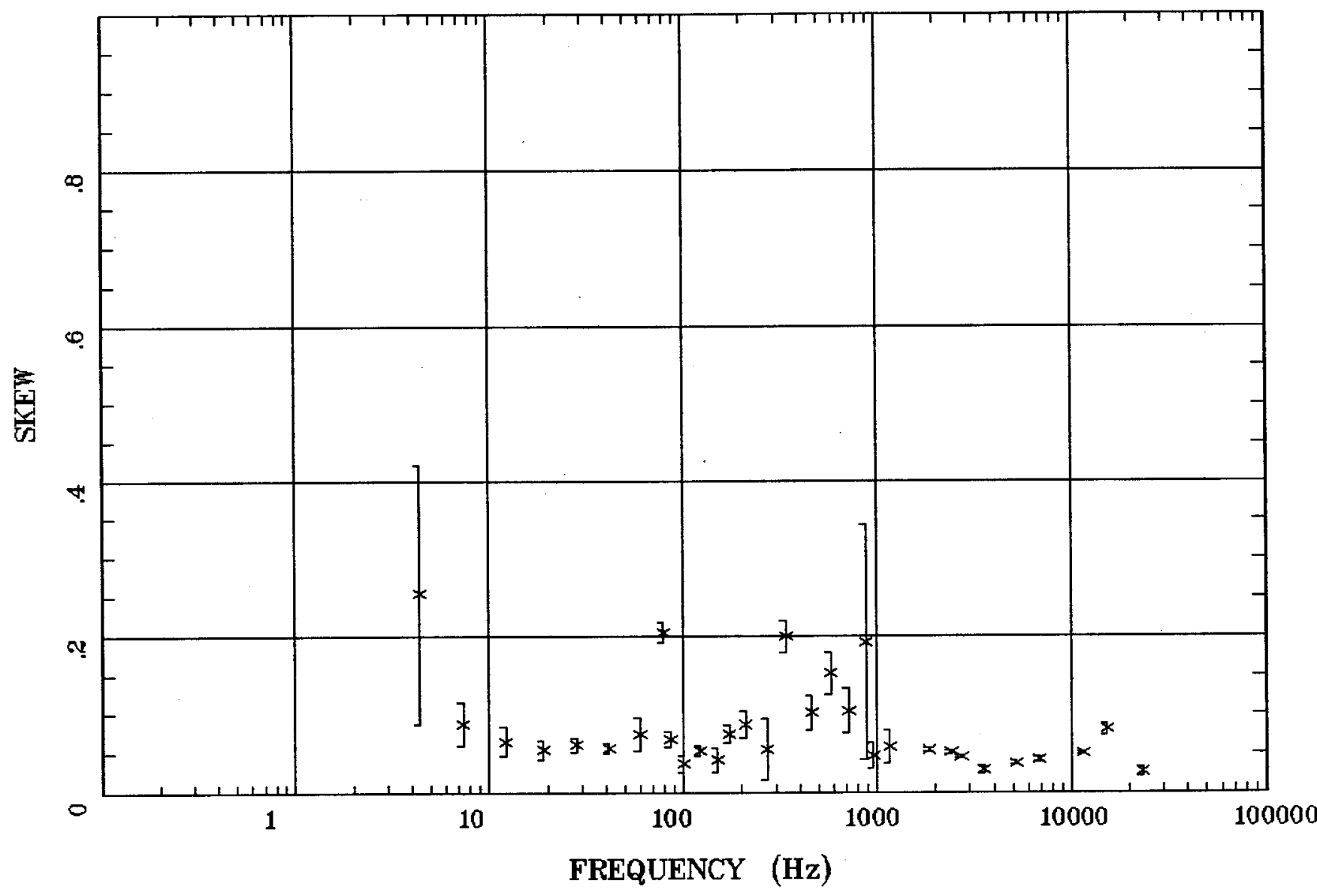

Client: Water Resources

Remote: none

Acquired: 11:1 Dec 03, 2003

Survey Co:USGS
Rotation:

Filename: nts8.avg

Channels: Ch1 Ch2 Ch3 Ch4 Ch5 Ch3 Ch4 Plotted: 08:47 Dec 07, 2004

< EMI - ElectroMagnetic Instruments 
E MULT Coh.

\section{Priority 6}

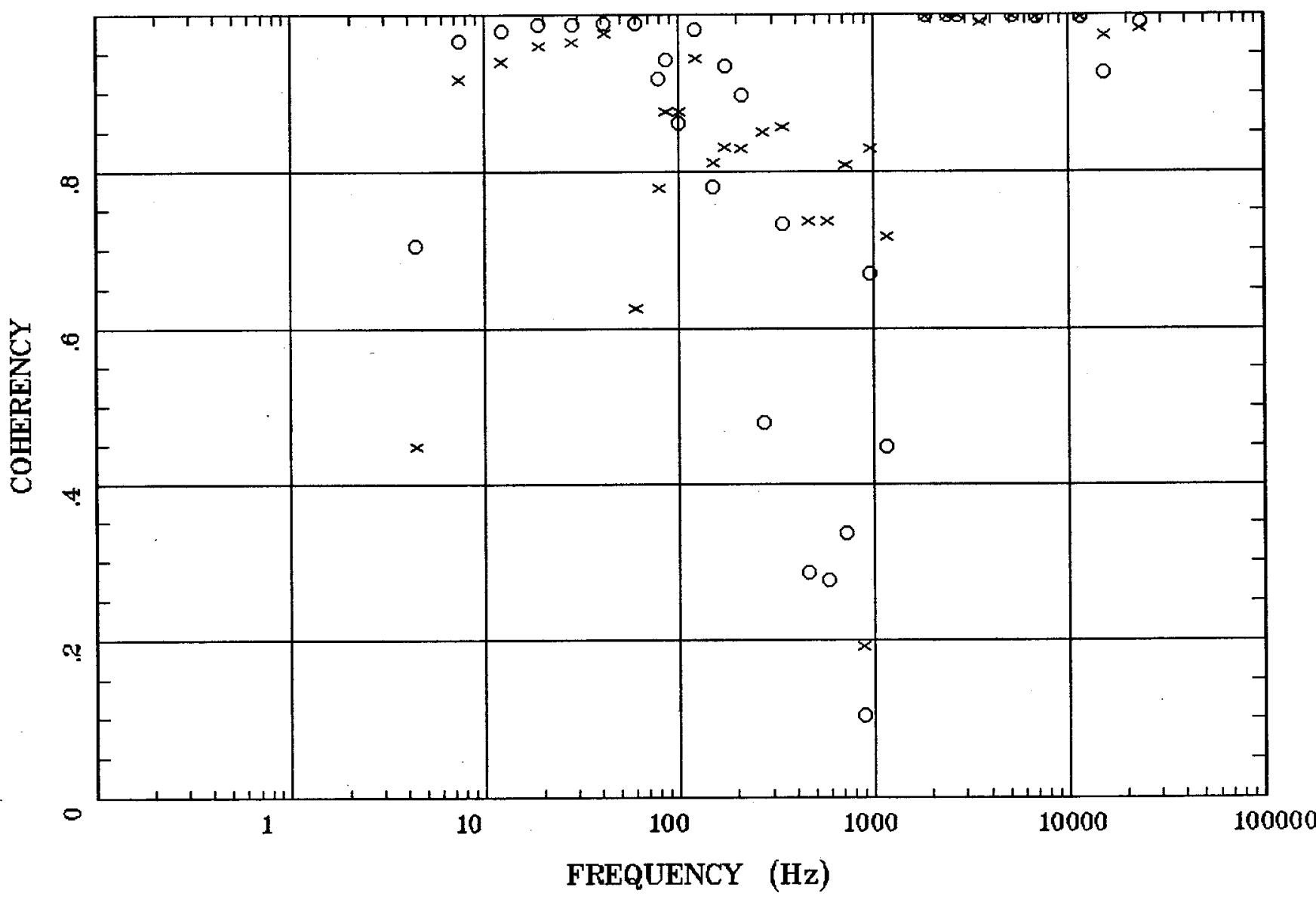

Client: Water Resources

Remote: none

Acquired: 11:1 Dec 03, 2003

Survey Co:USGS
Rotation:

Filename: nts6.avg

Channels: Ch1 Ch2 Ch3 Ch4 Ch5 Ch3 Ch4

Plotted: 08:47 Dec 07, 2004

< EMI - ElectroMagnetic Instruments > 


\section{POLAR PLOTS}

Priority 6

Station 8

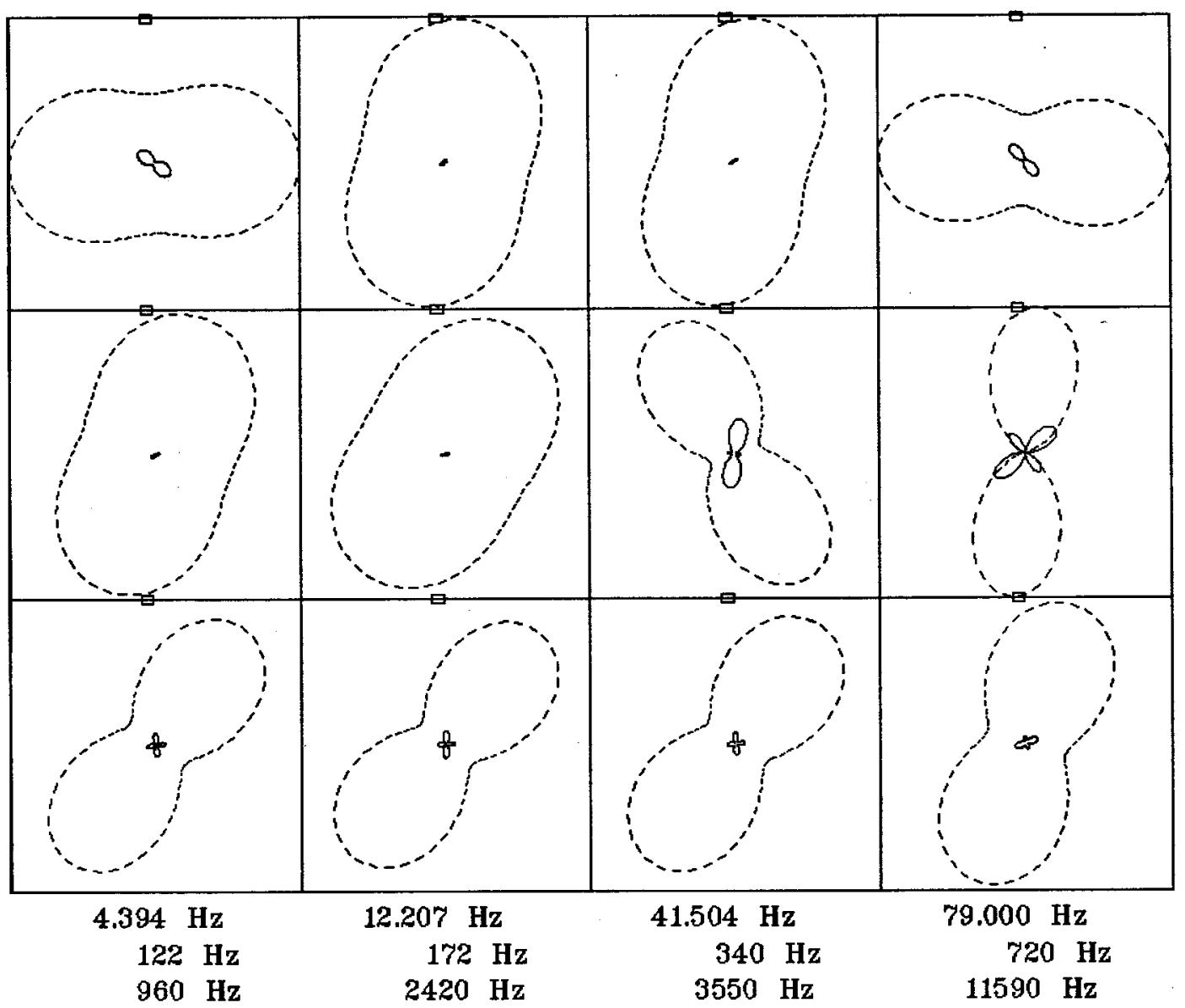

Rotation:

Client: Water Resources Remote: none

Acquired: 11:1 Dec 03, 2003 Survey Co:USGS
Filename: nts8.avg

Channels: Ch1 Ch2 Ch3 Ch4 Ch5 Ch3 Ch4 Plotted: 08:47 Dec 077, 2004

< EMI - ElectroMagnetic Instruments > 


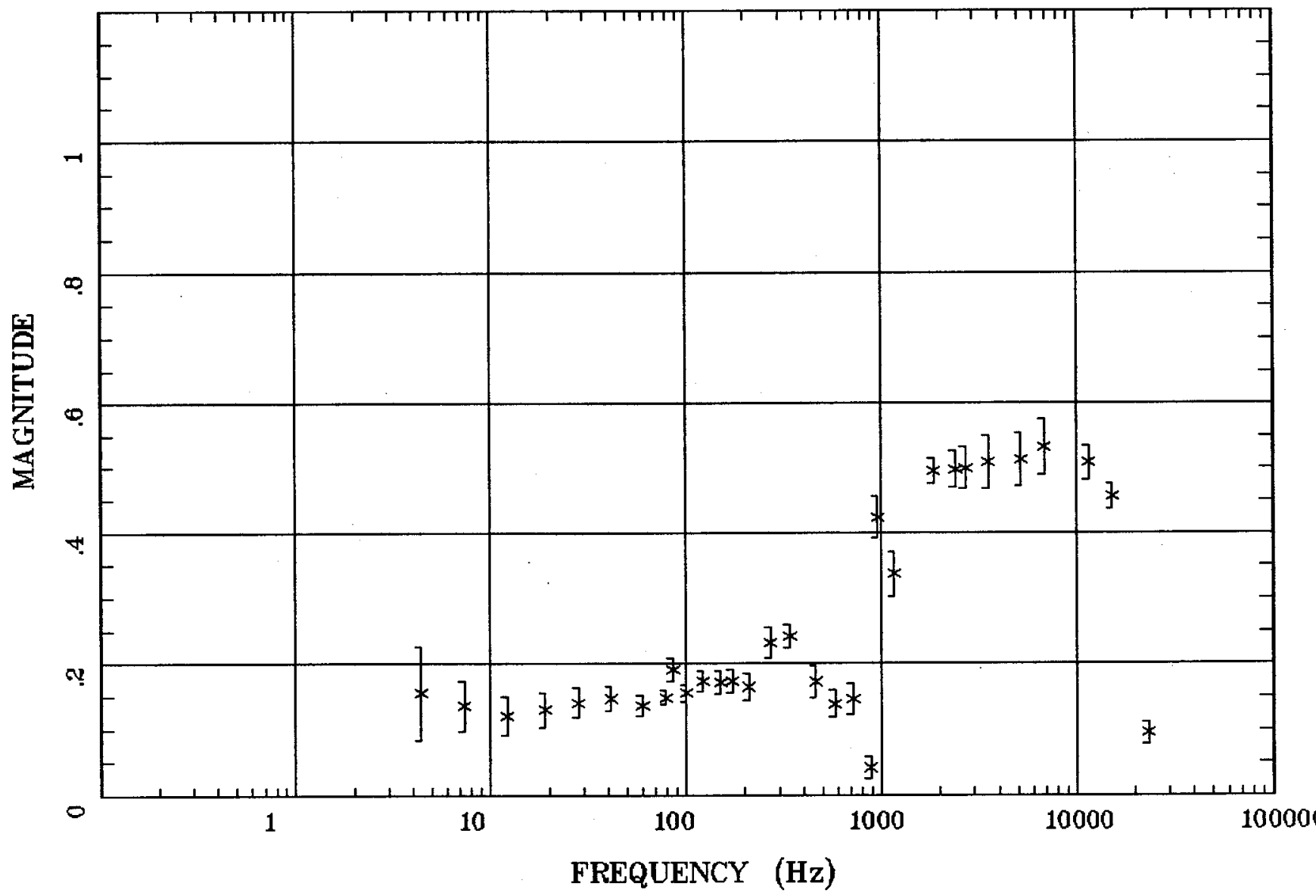

Client: Water Resources Remote: none Acquired: 11:1 Dec 03, 2003 Survey Co:USGS
Rotation:

Filename: nts8.avg

Channels: Ch1 Ch2 Ch3 Ch4 Ch5 Ch3 Ch4 Plotted: 08:47 Dec 07, 2004

$<$ EMI - ElectroMagnetic Instruments 


\section{TIPPER STRIKE}

\section{Priority 6}

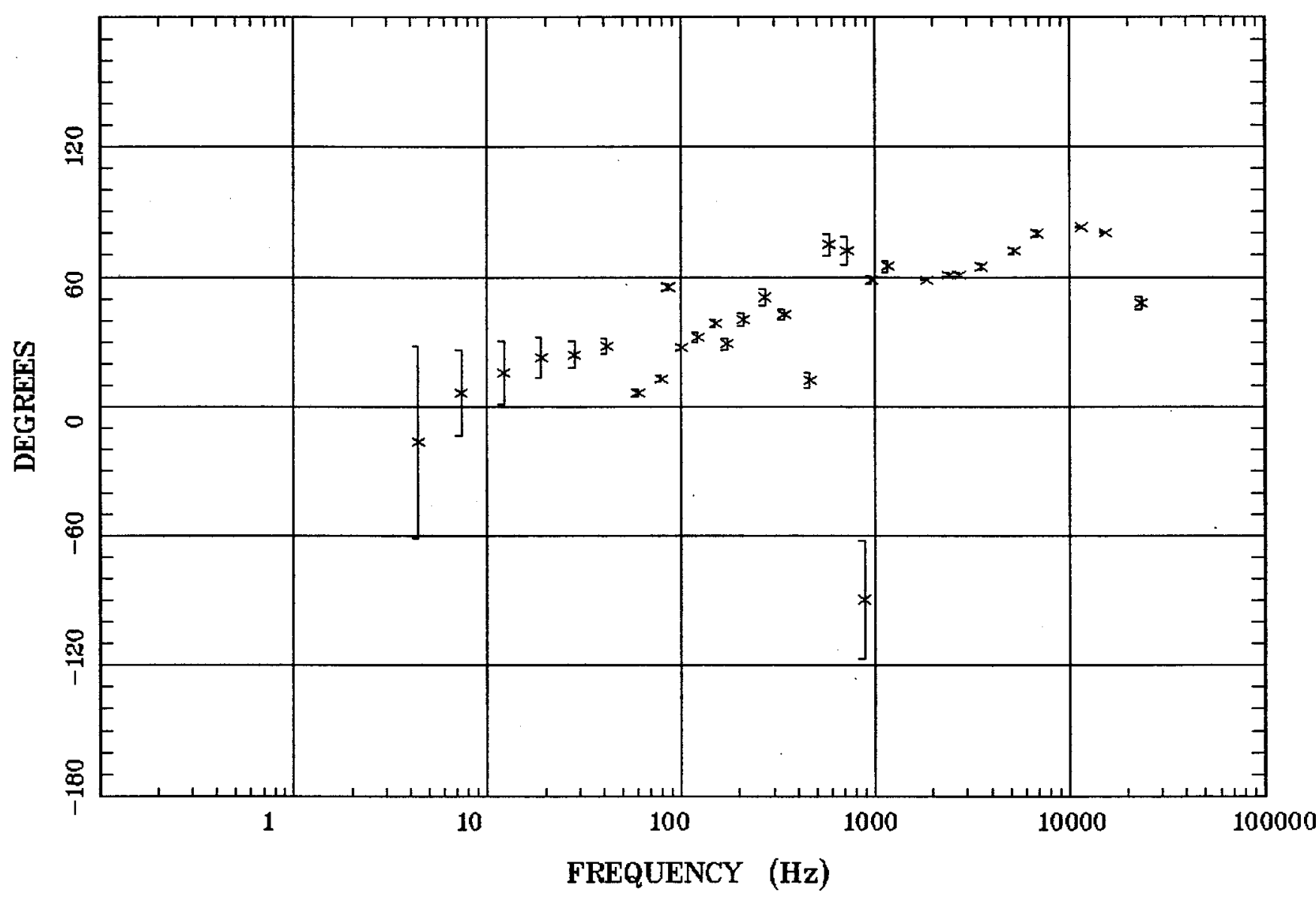

Client: Water Resources

Remote: none

Acquired: 11:1 Dec 03, 2003

Survey Co:USGS
Rotation:

Filename: nts8.avg

Channels: Ch1 Ch2 Ch3 Ch4 Ch5 Ch3 Ch4

Plotted: 08:47 Dec 07, 2004

< EMI - ElectroMagnetic Instruments > 


\section{HzHx.x Coh HzHy.o \\ Priority 6}

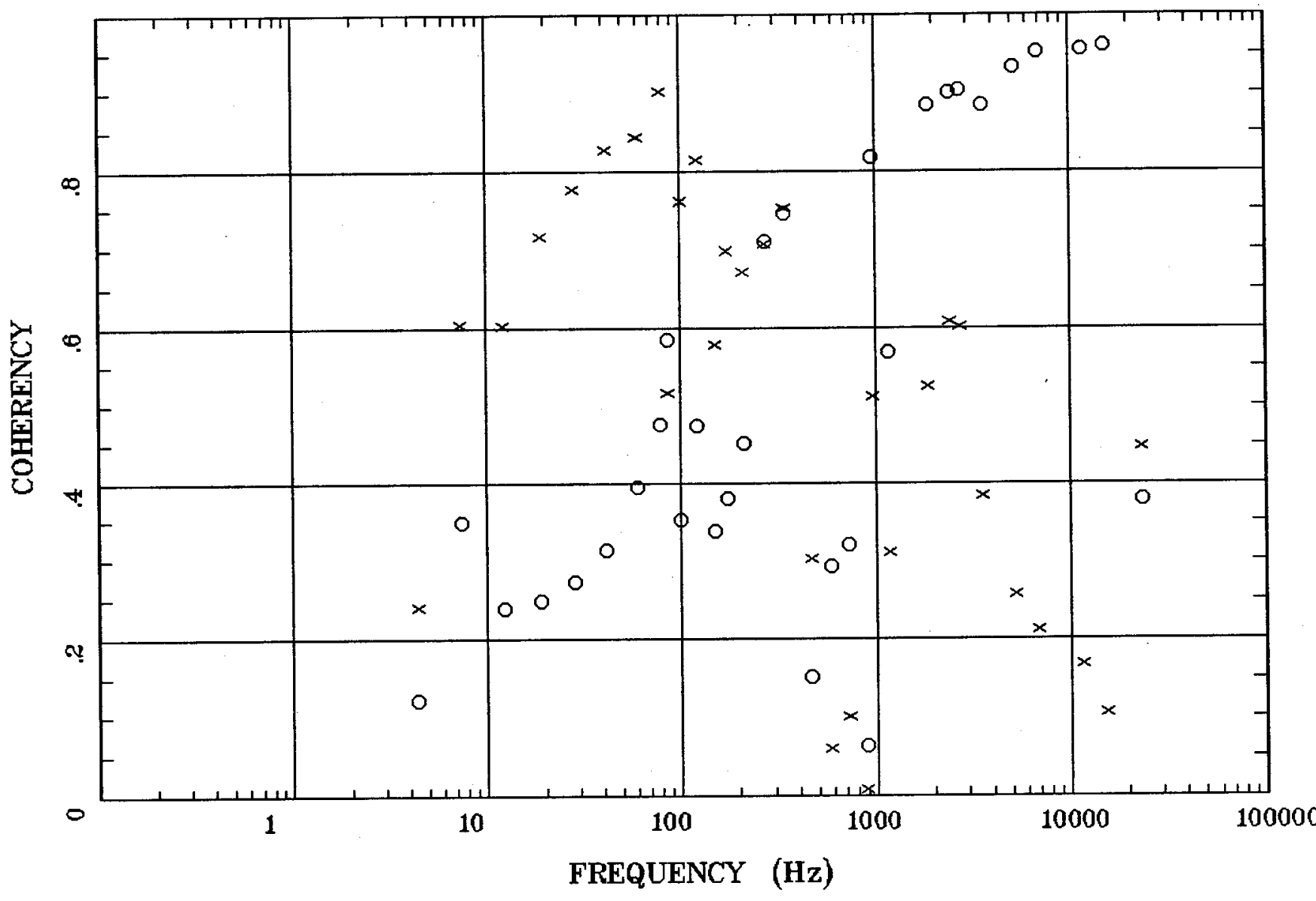

Client: Water Resources Remote: none Acquired: 11:1 Dec 03, 2003 Survey Co:USGS
Rotation:

Filename: nts8.avg

Channels: Ch1 Ch2 Ch3 Ch4 Ch5 Ch3 Ch4 Plotted: 08:47 Dec 07, 2004

$<$ EMI - ElectroMagnetic Instruments 